Georgia State University

ScholarWorks @ Georgia State University

Fall 12-14-2011

\title{
Arylboronic Acids With Strong Fluorescence Intensity Changes Upon Sugar Binding
}

Sarah R. Laughlin

Georgia State University, slaughlin1@student.gsu.edu

Follow this and additional works at: https://scholarworks.gsu.edu/chemistry_theses

\section{Recommended Citation}

Laughlin, Sarah R., "Arylboronic Acids With Strong Fluorescence Intensity Changes Upon Sugar Binding." Thesis, Georgia State University, 2011.

doi: https://doi.org/10.57709/2375571

This Thesis is brought to you for free and open access by the Department of Chemistry at ScholarWorks @ Georgia State University. It has been accepted for inclusion in Chemistry Theses by an authorized administrator of ScholarWorks @ Georgia State University. For more information, please contact scholarworks@gsu.edu. 


\title{
ARYLBORONIC ACIDS WITH STRONG FLUORESCENCE INTENSITY CHANGES UPON \\ SUGAR BINDING
}

by

SARAH R. LAUGHLIN

Under the Direction of Dr. Binghe Wang

\begin{abstract}
Boronic acids play an important role in the design and synthesis of chemosensors for carbohydrates due to their ability to reversibly bind with diol-containing compounds. Along this line, the availability of boronic acids that change fluorescence upon sugar binding is critical to a successful sensor design effort. Here, two boronic acids that show strong fluorescent intensity changes upon sugar binding are reported: isoquinoline-7-boronic acid (7-IQBA) and phenoxathiin-4-boronic acid (4-POBA).
\end{abstract}

INDEX WORDS: Chemosensor, Boronic acids, Saccharide recognition, Isoquinoline-7-boronic acid, Phenoxathiin-4-boronic acid, Fluorescence spectroscopy 


\section{ARYLBORONIC ACIDS WITH STRONG FLUORESCENCE INTENSITY CHANGES UPON \\ SUGAR BINDING}

by

SARAH R. LAUGHLIN

A Thesis Submitted in Partial Fulfillment of the Requirements for the Degree of

Master of Science

in the College of Arts and Science

Georgia State University

2011 
Copyright by

Sarah R. Laughlin

2011 


\section{ARYLBORONIC ACIDS WITH STRONG FLUORESCENCE INTENSITY CHANGES UPON \\ SUGAR BINDING}

by

SARAH R. LAUGHLIN

Committee Chair: Dr. Binghe Wang

Committee: Dr. Kathy Grant

Dr. Zhen Huang

Electronic Version Approved:

Office of Graduate Studies

College of Arts and Science

Georgia State University

December 2011 


\section{DEDICATION}

To my mother and sister, both of whom have supported me throughout my academic endeavors

and

In loving memory of my father, Darrell Lafain Laughlin, who passed away on May 4, 2011. I only hope I made you as proud of me, as I am of you. For a degree that meant so much to you, this is our achievement. 


\section{ACKNOWLEDGEMENTS}

A great deal of admiration goes to Dr. Binghe Wang for his exceptional leadership during the pursuit of this degree. I would especially like to thank my mentor, Dr. Nanting Ni, for her constant guidance and kind words. A large portion of this would not have been possible without the assistance of Mr. Yingji Wang and a special thank you to Mr. Weixuan Chen for his help with the molecular modeling. I would also to acknowledge the rest of my fellow group members, whom have helped me mature both academically and personally. 


\section{TABLE OF CONTENTS}

ACKNOWLEDGEMENTS $\quad$ v

LIST OF FIGURES $\quad$ viii

LIST OF SCHEMES $\quad$ ix

LIST OF TABLES $\quad$ x

LIST OF EQUATIONS $\quad$ xi

1 INTRODUCTION 1

1.1 CURRENT DRug DESIGN 1

$\begin{array}{lll}1.2 & \text { BORONIC ACIDS } & 1\end{array}$

1.3 Saccharide Detection USING Boronic Acids 2

1.4 PURPOSE OF THIS STUDY $\quad 2$

2 RESULTS \& DISCUSSION

2.1 Apparent Association Constants $\left(K_{\mathrm{a}}\right) \quad \mathbf{5}$

$\begin{array}{lll}2.2 & \text { FLUORESCENCE INTENSITY CHANGES } & 9\end{array}$

2.2.1 Isoquinoline-7-Boronic Acid 9

2.2.2 Phenoxathiin-4-Boronic Acid 12

$\begin{array}{lll}2.3 & \text { IONIZATION STATES } & 17\end{array}$

$\begin{array}{ll}\text { 2.3.1 Isoquinoline-7-Boronic Acid } & 17\end{array}$

2.3.2 Phenoxathiin-4-Boronic Acid $\quad 24$

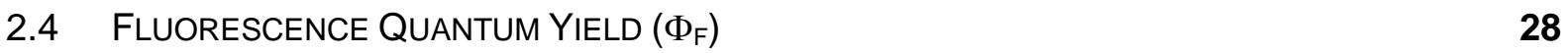


3 CONCLUSIONS

4 EXPERIMENTAL $\quad 33$

5 NOTES AND REFERENCES $\quad 35$

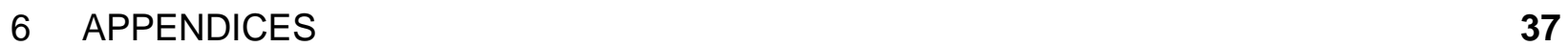

$\begin{array}{lll}\text { APPENDIX A } & \text { Apparent Association Constants }\left(K_{\mathrm{a}}\right) & 37\end{array}$

$\begin{array}{lll}\text { APPENDIX B } & \text { Fluorescence Quantum Yield }\left(\Phi_{\mathrm{F}}\right) & 47\end{array}$

$\begin{array}{lll}\text { APPENDIX C } & \mathrm{pH} \text { Profiles \& Identification of } \mathrm{p} K_{\mathrm{a}} \text { Values } & \mathbf{5 8}\end{array}$

APPENDIX D Titration Reports for Identification of $p K_{\mathrm{a}}$ Values $\quad 69$ 


\section{LIST OF FIGURES}

Figure 1. Structures of 7-IQBA and 4-POBA

Figure 2. D-Saccharides used

$\begin{array}{llr}\text { Figure 3. } & \text { Absorbance spectra of boronic acids } & 6\end{array}$

Figure 4. Fluorescence spectra of 7-IQBA upon addition of sugar 10

Figure 5. Fluorescence intensity changes of 7-IQBA upon addition of sugar

Figure 6. Fluroescence spectra of 4-POBA upon addition of sugar

Figure 7. Fluorescence intensity changes of 4-POBA upon addition of sugar

Figure 8. Molecular models for intramolecular hydrogen bond formation of 4-POBA

Figure 9. $\mathrm{pH}$ profile of 7-IQBA using $\lambda_{\mathrm{em}}=344 \mathrm{~nm}$

Figure 10. $\mathrm{pH}$ profile of 7-IQBA using $\lambda_{\mathrm{em}}=378 \mathrm{~nm}$

Figure 11. $\mathrm{pH}$ profile of 4-POBA using $\lambda_{\mathrm{em}}=430 \mathrm{~nm}$

Figure 12. Fluroescence quantum yield slopes of 7-IQBA and reference

Figure 13. Evidence of species aggregation 


\section{LIST OF SCHEMES}

Scheme 1. Proposed ionization of 7-IQBA in the absence of sugar

Scheme 2. Proposed ionizations of 7-IQBA in the presence of a sugar

Scheme 3. Proposed ionization of 4-POBA in the absence of sugar

Scheme 4. Proposed ionization of 4-POBA in the presence of sugar 


\section{LIST OF TABLES}

Table 1. Apparent association constants $\left(K_{\mathrm{a}}\right)$ of 7-IQBA and 4-POBA

Table 2. Apparent $p K_{\mathrm{a}}$ values of 7-IQBA acid and 4-POBA

Table 3. Presumed structures of 7-IQBA and its sugar esters at $\mathrm{pH} 7.4$

Table 4. Presumed structures of 4-POBA and its sugar esters at $\mathrm{pH} 7.4$

Table 5. Fluorescence quantum yields $\left(\Phi_{\mathrm{F}}\right)$ of 7-IQBA and 4-POBA

30 


\section{LIST OF EQUATIONS}

$\begin{array}{lll}\text { Equation 1. } & \text { Apparent association constant }\left(K_{\mathrm{a}}\right) & \mathbf{7}\end{array}$

$\begin{array}{lll}\text { Equation 2. (a) } \mathrm{p} K_{\mathrm{a}} \text { value curve fitting calculation from Sigma Plot } 10.0 & \mathbf{1 7}\end{array}$

(b) $\mathrm{p} K_{\mathrm{a}}$ value curve fitting calculation from Sigma Plot 12.0

$\begin{array}{lll}\text { Equation 3. } & \text { Fluorescence quantum yield }\left(\Phi_{F}\right) & \mathbf{2 8}\end{array}$

$\begin{array}{lll}\text { Equation 4. } & \mathrm{pH} 7.4 \text { Fluorescence quantum yield ratio used for this study } & \mathbf{2 8}\end{array}$ 


\section{INTRODUCTION}

\subsection{Current Drug Design}

Health issues such as monitoring blood glucose for people with diabetes mellitus ${ }^{1}$ and cancer diagnosis are current areas of interest. ${ }^{2}$ In drug discovery, screening and computational methods are often employed to identify potential drug molecules from libraries of enzyme inhibitors. ${ }^{3}$ Other prospects in drug development focus on gene therapy to recognize specific sequences of nucleic acids to treat metabolic disorders, cancers, and human immunodeficiency virus. $^{4}$ Designing receptors for the specific purpose of diagnostic and therapeutic effects remains a challenge. Structure, stereochemistry, and polarity are only a few factors that influence the reactivity of a receptor. The development and success of marketed drugs is based on their selective recognition for target molecules.

\subsection{BORONIC ACIDS}

Boronic acids are often used in the syntheses of heterocyclic and aryl-substituted compounds. ${ }^{5}$ Aside from their use in organic syntheses, they have also been used to recognize adenosine triphosphate (ATP) and nucleic acids, ${ }^{6}$ serine protein motifs, ${ }^{7} \alpha$-hydroxy carboxylic acids, ${ }^{8}$ and fluoride ions. ${ }^{9}$ They are often found in a trigonal planar neutral form, but can easily convert to a tetrahedral anion at $\mathrm{pH}$ above the $\mathrm{p} K_{\mathrm{a}}$ for a particular species. This is due to the fact that boron behaves as a Lewis acid and accepts lone pair electrons from donating atoms to form covalent bonds. The boronic acid group can form tight complexes with compounds that contain two adjacent nucleophiles, especially cis-diols. With a high intrinsic affinity for sugars and a rapid, reversible equilibrium, boronic acids continue to gain more interests in sensor scaffold design for the recognition of carbohydrates. ${ }^{7 \mathrm{c}, 10}$ 


\subsection{SAccharide Detection Using Boronic Acids}

Carbohydrates are known to be extremely important in many biochemical processes and are endogenous in even the simplest of cells. ${ }^{11}$ Carbohydrates are often linked to cell surfaces and proteins which can affect activity. ${ }^{12}$ For example, the carbohydrate chain of a red blood cell is what determines blood type. Modified saccharide chains have been found in many degenerative diseases, ${ }^{12 a, 13}$ and glycosolated proteins have been shown to play roles in cell-cell communication and cancer metastasis. ${ }^{14}$

A large number of boronic acids have focused on sensing glucose and fructose because of their abundance in metabolic processes. However, the detection of other carbohydrates remains equally important. Therefore, the design and synthesis of boronic acids is important for specificity to sugars. Boronic acids are useful for sensing many types of saccharides and several boronic acids have been developed to recognize cell-surface carbohydrates.

\subsection{PuRpose OF ThIS Study}

Boronic acids are good fluorescence sensors because they have intrinsic affinity for carbohydrates, fluorescence intensity changes upon binding with sugars, and a rapid and reversible equilibrium. These studies can be performed in aqueous solution under physiological conditions. A long-term goal in our group is to create a library of boronic acid scaffolds with known values that can be later used for the design and synthesis of chemosensors to identify specific biomarkers. This work examines two commercially available aryl-boronic acids: isoquinoline-7-boronic acid and phenoxathiin-4-boronic acid. Our group recently reported a new class of issoquinoinyl boronic acids $(4,5,6,8$-IQBA) that were used for binding with sugars and six-member ring diols. 7-IQBA was not included in this earlier report, which therefore encouraged us to include it in this class of IQBAs. The structure of 4-POBA is comparatively different from IQBAs which enabled us to compare and contrast the two compounds. Binding 
affinities of boronic acid with saccharides were determined from calculated $K_{\mathrm{a}}$ values to show how tightly the boronic acids bind to sugars. The $p K_{a}$ values for each boronic acid in both the absence and presence of saccharides were determined to better understand the mechanism by which changes in fluorescence occurred. Additionally, fluorescence quantum yields $\left(\Phi_{\mathrm{F}}\right)$ of both boronic acids with and without sugar were used to illustrate the fluorescence efficiency associated for boronic acid and their saccharide esters. 
<smiles>OB(O)c1ccc2ccncc2c1</smiles>

Isoquinoline-7-boronic acid (1)<smiles>OB(O)c1cccc2c1Oc1ccccc1S2</smiles>

Phenoxathiin-4-boronic acid (2)

Figure 1. Structures of 7-IQBA and 4-POBA.<smiles>OC[C@H]1C[C@@](O)(CO)[C@@H](O)[C@@H]1O</smiles>

D-fructose<smiles>OC[C@@H](O)[C@H](O)[C@H](O)[C@H](O)CO</smiles>

D-sorbitol<smiles>OC[C@H]1O[C@H](O)[C@@H](O)[C@H](O)[C@@H]1O</smiles>

D-glucose<smiles>OC[C@H]1O[C@H](O)[C@@H](O)[C@H](O)[C@@H]1O</smiles>

D-mannose<smiles>O=C(CO)[C@H](O)[C@H](O)[C@H](O)CO</smiles>

D-tagatose

Figure 2. D-Saccharides used for this study. 


\section{RESULTS \& DISCUSSION}

\subsection{Apparent Association Constants $\left(K_{\mathrm{a}}\right)$}

Binding affinities were determined to illustrate how tightly boronic acids bind with sugars. To do this, binding affinities were determined from $K_{\mathrm{a}}$ values. The $K_{\mathrm{a}}$ values were calculated using a derived equation that correlates the fluorescence intensity changes to the concentration of sugar. Excitation wavelengths used for fluorescence emission were chosen based on the maximum intensity observed at a specific wavelength (Figure 3). Binding constants for boronic acids were determined by measuring the fluorescence intensity at the emission wavelength maximum upon addition of sugar.

The $K_{\mathrm{a}}$ value was calculated using a derived equation that correlates the fluorescence intensity changes to the concentration of sugar. The $K_{\mathrm{a}}$ value for boronic acid-sugar ester can be calculated from equation (1). At equilibrium, boronic acid-sugar complex concentration is $[B S]$, boronic acid concentration is $[\mathrm{B}]-[\mathrm{BS}]$, and sugar concentration is [S]-[BS]. Assume that the concentration of sugar is much larger than both the concentrations of boronic acid and boronic acid-sugar complex. Also assume that $[\mathrm{BS}]=n \Delta /$ and $[\mathrm{B}]=m l_{0}$. Following the derivation, the linear equation used to calculate $K_{\mathrm{a}}$ value is shown. 

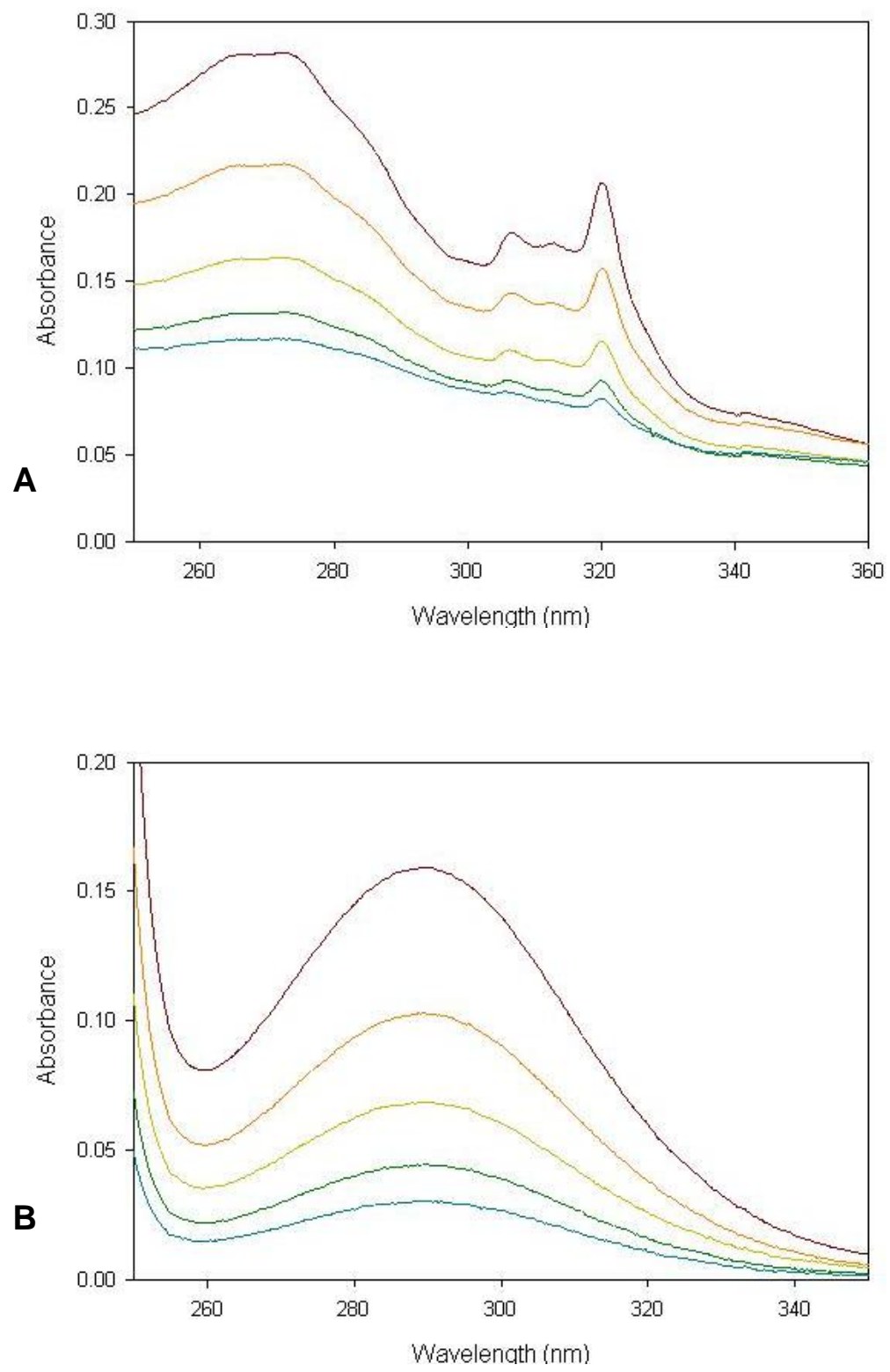

Figure 3. Absorbance spectra for boronic acid. Excitation wavelengths for fluorescence emission of boronic acids were chosen based on maximum absorbance intensity. Experiments were performed in phosphate buffer $(0.1 \mathrm{M})$, pH 7.4: 7-IQBA, $\lambda_{\max }=271 \mathrm{~nm}$; 4-POBA $\lambda_{\max }=299 \mathrm{~nm}$. A) 7-IQBA only, B) 4POBA only. 


$$
\begin{aligned}
& \text { Boronic acid + sugar } \longrightarrow \text { B-S Complex } \\
& {[B]} \\
& {[\mathrm{S}]} \\
& {[\mathrm{B}]-[\mathrm{BS}] \quad[\mathrm{S}]-[\mathrm{BS}]} \\
& \text { [BS] } \\
& \text { [BS] } \\
& {[B]=m I_{0},[B S]=n \Delta I} \\
& K_{a}=\frac{[B S]}{[B][S]}=\frac{[B S]}{([B][B S]) \cdot([S]-[\mathrm{BS}])} \\
& \therefore[S] \gg[B] \therefore[S] \gg[B S] \\
& K_{a}=\frac{[B S]}{([B]-[B S]) \cdot[S]} \\
& K_{a}=\frac{n \Delta I}{\left(m I_{0}-n \Delta I\right) \cdot[S]} \\
& K_{a}[S] \cdot\left(m I_{0}-n \Delta I\right)=n \Delta I \\
& K_{a}[S]\left(m I_{0}\right)-K_{a}[S](n \Delta I)=n \Delta I \\
& K_{a}[S]\left(m I_{0}\right)=K_{a}[S](n \Delta I)+n \Delta I \\
& K_{a}[S]\left(m I_{0}\right)=\left(K_{a}[S]+1\right) \cdot n \Delta I \\
& \frac{K_{a}[S]\left(m I_{0}\right)}{\Delta I}=\left(K_{a}[S]+1\right) \cdot n \\
& \frac{I_{0}}{\Delta I}=\frac{\left(K_{a}[S]+1\right) \cdot n}{m \cdot K_{a}[S]} \\
& \frac{I_{0}}{\Delta I}=\frac{n}{m K_{a}} \cdot \frac{1}{[S]}+\frac{n}{m} \\
& y=a \cdot x+b \\
& K_{a}=\frac{b}{a}=\frac{n / m}{n / m K_{a}}=K_{a}
\end{aligned}
$$


The binding affinities of 7-IQBA with D-sorbitol, D-tagatose, D-mannose, and D-fructose are listed in Table 1.The trend for the binding affinities of 7-IQBA began with D-sorbitol $\left(1600 \mathrm{M}^{-1}\right)$, followed by D-fructose $\left(1100 \mathrm{M}^{-1}\right)$, D-tagatose $\left(620 \mathrm{M}^{-1}\right)$, and D-mannose $\left(58 \mathrm{M}^{-1}\right)$. The fluorescence intensity of 7-IQBA with D-glucose was low, even as sugar concentration increased. The $K_{\mathrm{a}}$ value between 7-IQBA with D-glucose was not detected. The binding affinities of 4-POBA with saccharides varied slightly in order of binding and is also listed in Table 1. Binding between 4-POBA with D-sorbitol had the highest $K_{\mathrm{a}}$ value $\left(740 \mathrm{M}^{-1}\right)$. The trend followed with D-tagatose $\left(520 \mathrm{M}^{-1}\right)$, D-fructose $\left(370 \mathrm{M}^{-1}\right)$, D-mannose $\left(31 \mathrm{M}^{-1}\right)$, and finally D-glucose $\left(14 \mathrm{M}^{-1}\right)$.

Table 1. Apparent association constants $\left(K_{\mathrm{a}}\right)$ of 7-IQBA and 4-POBA.

\begin{tabular}{ccccc}
\hline & \multicolumn{2}{c}{ 7-IQBA } & \multicolumn{2}{c}{ 4-POBA } \\
& $K_{\mathrm{a}}\left[\mathrm{M}^{-1}\right]$ & $\Delta \mathrm{l} / \mathrm{I}_{0}$ & $K_{\mathrm{a}}\left[\mathrm{M}^{-1}\right]$ & $\Delta \mathrm{l} / \mathrm{I}_{0}$ \\
\hline D-fructose & $1100 \pm 10$ & 20.0 & $370 \pm 82$ & -0.72 \\
D-glucose & \multicolumn{2}{c}{ Not Detected } & $14 \pm 2$ & -0.90 \\
D-mannose & $58 \pm 23$ & 5.1 & $31 \pm 7$ & -0.82 \\
D-sorbitol & $1600 \pm 588$ & 8.0 & $740 \pm 68$ & -0.81 \\
D-tagatose & $620 \pm 38$ & 6.9 & $520 \pm 78$ & -0.78 \\
\hline
\end{tabular}




\subsection{FLuORESCENCE INTENSITY ChANGES}

\subsubsection{Isoquinoline-7-Boronic Acid}

Fluorescence intensity changes of 7-IQBA upon sugar binding are listed in Table 1. The changes ranged from the intensity in the absence of sugar (initial intensity, $I_{0}$ ) to the intensity in the highest concentration of sugar (final intensity, $I_{F}$ ). The intensities of 7-IQBA upon binding with saccharides increased, except for 7-IQBA with D-glucose. In particular, 7-IQBA showed a 20fold increase in fluorescence intensity upon binding with D-fructose. Changes in fluorescence intensities of 7-IQBA experienced a 5 to 8-fold increase with the remaining sugars (Figures 4 and 5).The maximum fluorescence intensity red-shifted from around $344 \mathrm{~nm}$ to $378 \mathrm{~nm}$ as saccharide concentrations increased (Figure 4).

The fluorescence intensity of 7-IQBA only is lower than its sugar esters. At pH 7.4, 7IQBA is predominantly found as a neutral trigonal planar species and its ester is the tetrahedral anionic form at $\mathrm{pH}$ 7.4. The ester form is the fluorescent species while 7-IQBA is nonfluorescent at $\mathrm{pH}$ 7.4. Therefore, the fluorescence intensities increase upon addition of sugar. These results are further explained with apparent $p K_{\mathrm{a}}$ values. 

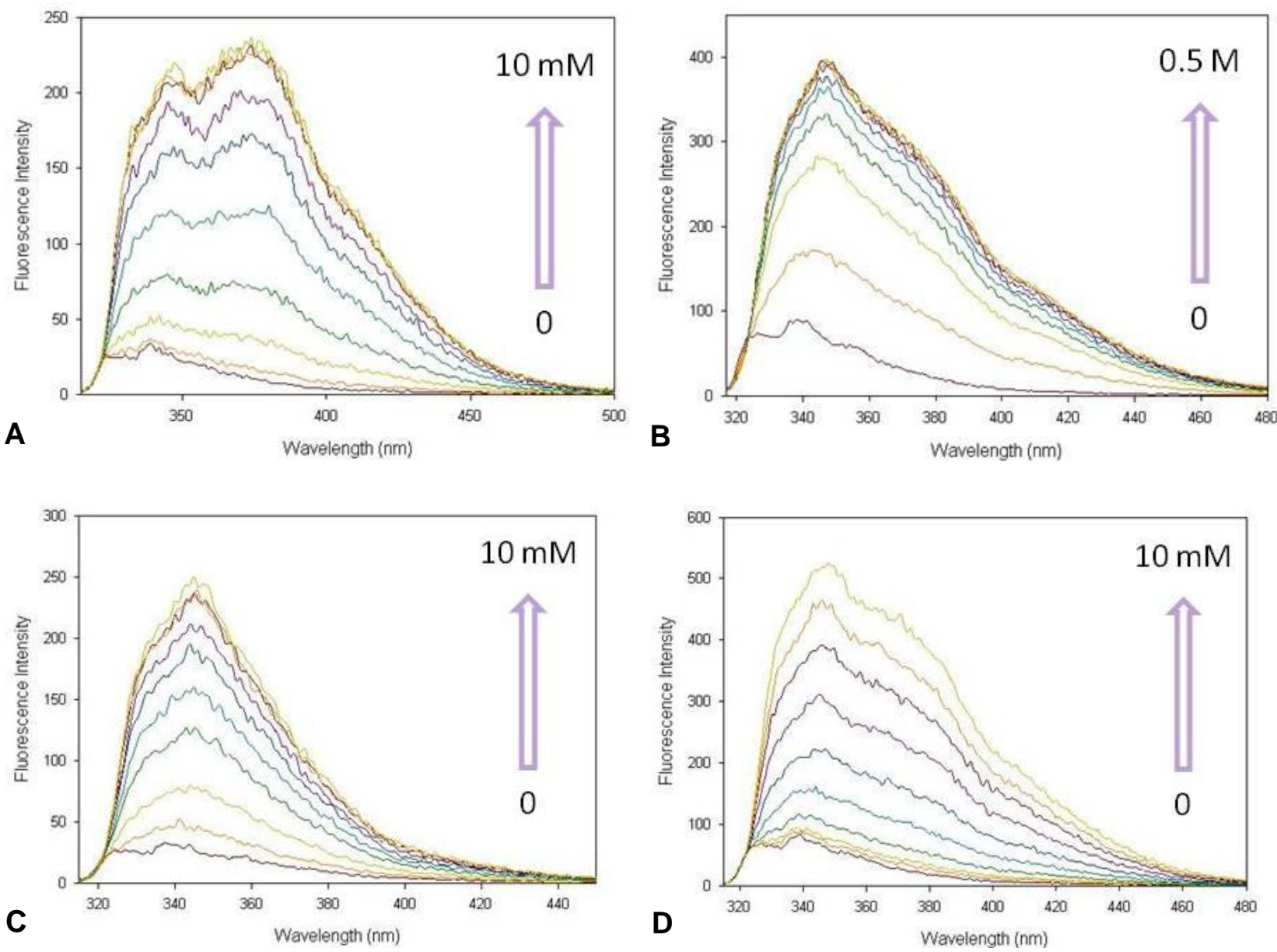

Figure 4. Fluorescence spectra of 7-IQBA $(10 \mu \mathrm{M})$ upon addition of increasing sugar concentrations in phosphate buffer $(0.1 \mathrm{M})$ at $\mathrm{pH}$ 7.4: $\lambda_{\mathrm{ex}}=280 \mathrm{~nm}$, D-fructose and D-sorbitol; $\lambda_{\mathrm{ex}}=272 \mathrm{~nm}$, D-mannose and D-tagatose. All experiments were performed in triplicate. A) D-fructose, B) D-mannose, C) D-sorbitol, D) Dtagatose. 


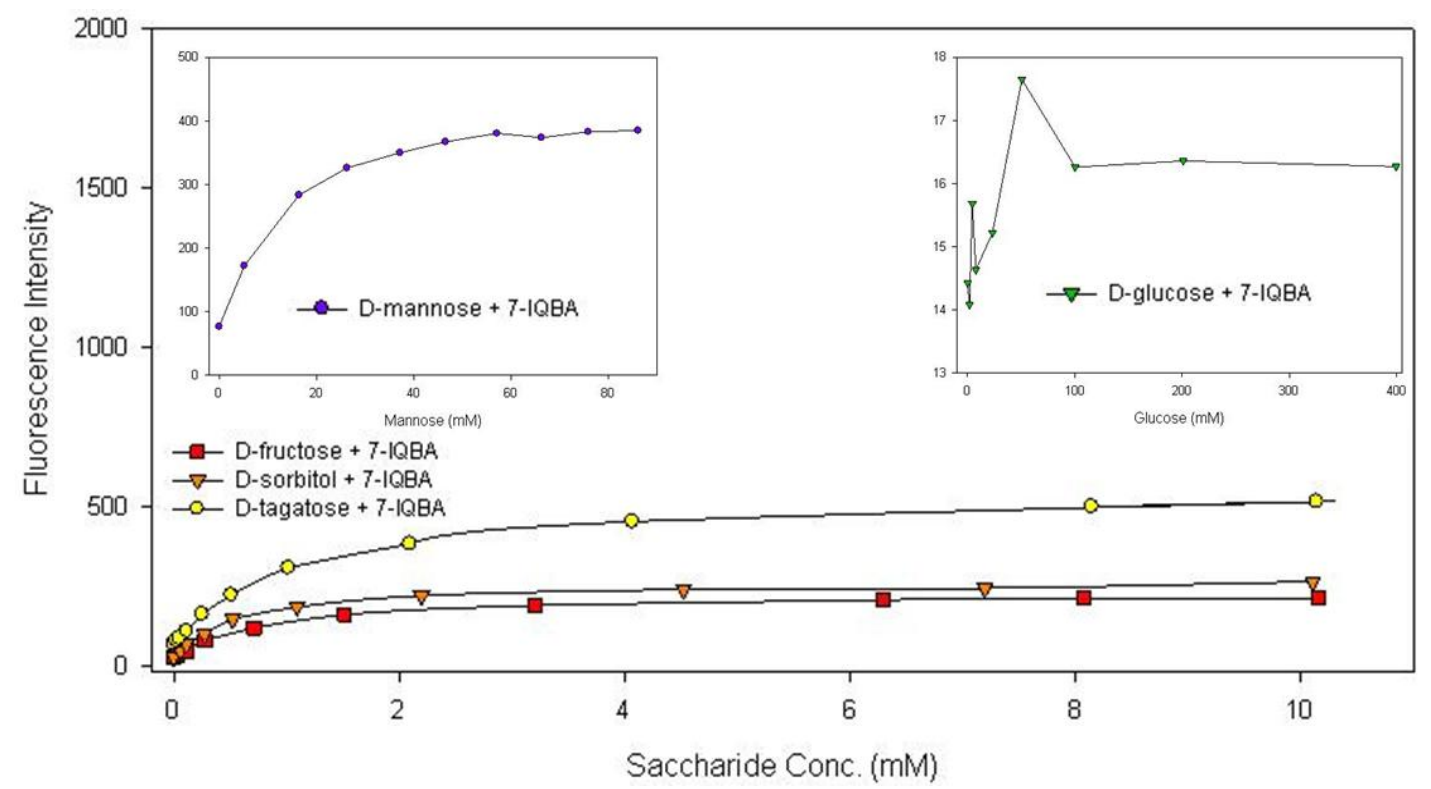

Figure 5. Fluorescence intensity changes of 7-IQBA $(10 \mu \mathrm{M})$ upon increasing sugar concentration in phosphate buffer $\left(0.1 \mathrm{M}\right.$ ) at $\mathrm{pH} 7.4: \lambda_{\mathrm{ex}}=280 \mathrm{~nm}, \lambda_{\mathrm{em}}=370 \mathrm{~nm}$ (D-fructose) and $\lambda_{\mathrm{em}}=344 \mathrm{~nm}$ (D-sorbitol); $\lambda_{\mathrm{ex}}=272 \mathrm{~nm}, \lambda_{\mathrm{em}}=347 \mathrm{~nm}$ (D-mannose) and $\lambda_{\mathrm{em}}=346 \mathrm{~nm}$ (D-tagatose). All experiments were performed in triplicate. 


\subsubsection{Phenoxathiin-4-Boronic Acid}

The fluorescence intensity of 4-POBA upon addition of saccharides decreased. The changes are listed in Table 1. Addition of D-glucose with 4-POBA produced the largest intensity decrease by $90 \%$. In the presence of D-mannose, D-sorbitol, or D-tagatose, the intensities decreased $80 \%$. A decrease of $72 \%$ showed that binding between $4-P O B A$ and D-fructose experienced the smallest change in fluorescence. 

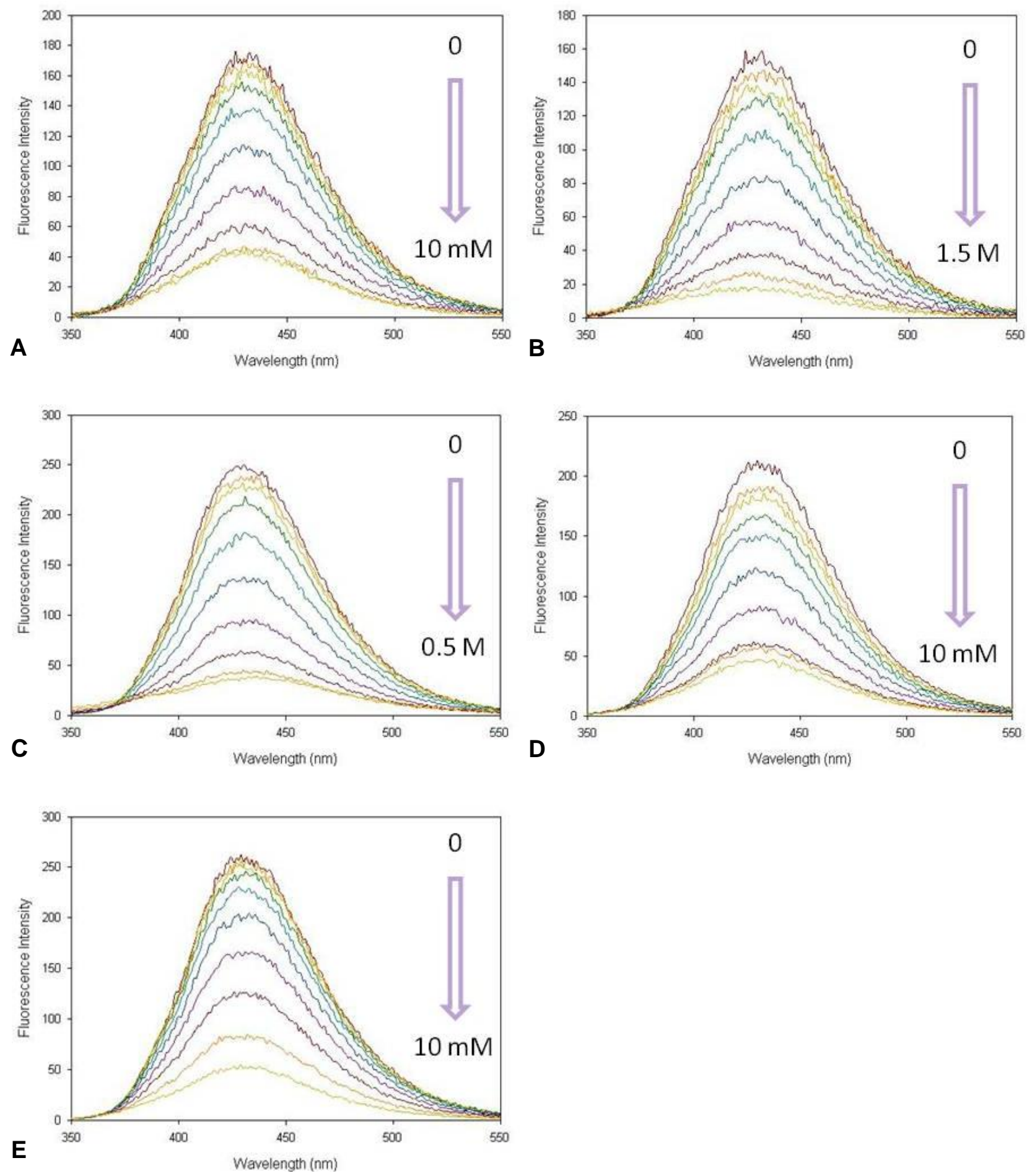

Figure 6. Fluorescence spectra of 4-POBA $(10 \mu \mathrm{M})$ upon addition of increasing sugar concentration in phosphate buffer $(0.1 \mathrm{M})$ at $\mathrm{pH} 7.4: \lambda_{\mathrm{ex}}=299 \mathrm{~nm}$, all sugars. All experiments were performed in triplicate. A) D-fructose, B) D-glucose, C) D-mannose, D) D-sorbitol, E) D-tagatose. 


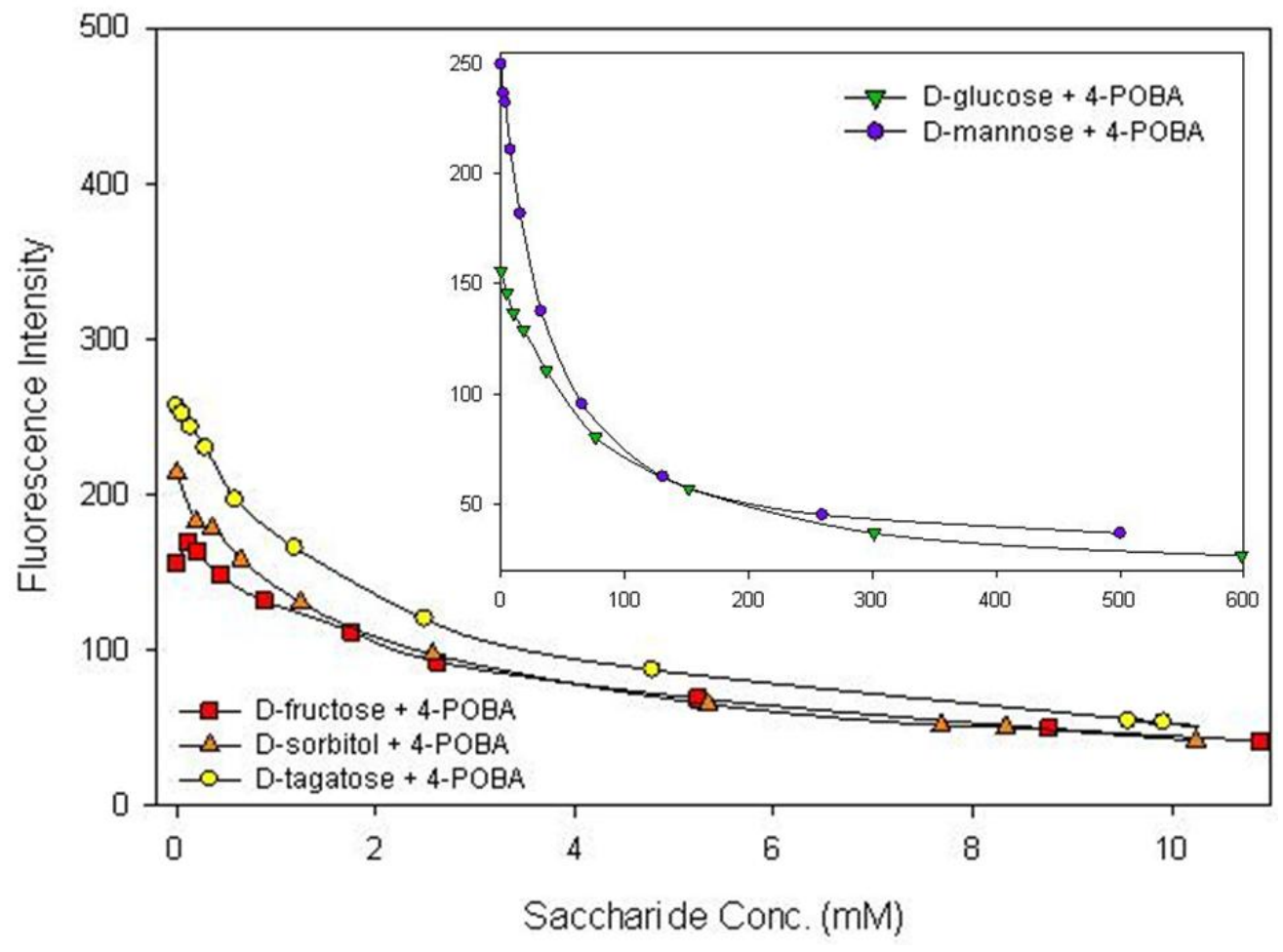

Figure 7. Fluorescence intensity changes of 4-POBA $(10 \mu \mathrm{M})$ upon increasing sugar in phosphate buffer $(0.1 \mathrm{M})$ at $\mathrm{pH}$ 7.4: $\lambda_{\mathrm{ex}}=299 \mathrm{~nm}, \lambda_{\mathrm{em}}=430 \mathrm{~nm}$ (D-fructose, D-glucose, D-mannose, D-sorbitol, and Dtagatose). All experiments were performed in triplicate. 
For 4-POBA, the fluorescence intensity decreased upon increasing $\mathrm{pH}$ values of free boronic acid. The measured distance between the oxygen of the phenoxathiin moiety and hydrogen of boronic acid was $2.04 \AA$. Intrinsic distance for hydrogen bonding is roughly $2 \AA$. We can predict that there is hydrogen bond interaction between these two groups. In the ester form, $\mathrm{pH}$ of the solution is higher than the $\mathrm{pK}_{\mathrm{a}}$ value of 4-POBA-sugar esters and the hybridization changes from trigonal planar to tetrahedral anionic form. There is no hydrogen on the boronic ester, thus no hydrogen bonding will exist. Hydrogen bonding between the free boronic acid and oxygen may contribute to the stability of the boronic acid and to the fluorescence intensity. We can speculate that breaking this hydrogen bond may play a role in lowering the fluorescence intensity upon sugar addition or with increasing $\mathrm{pH}$. 

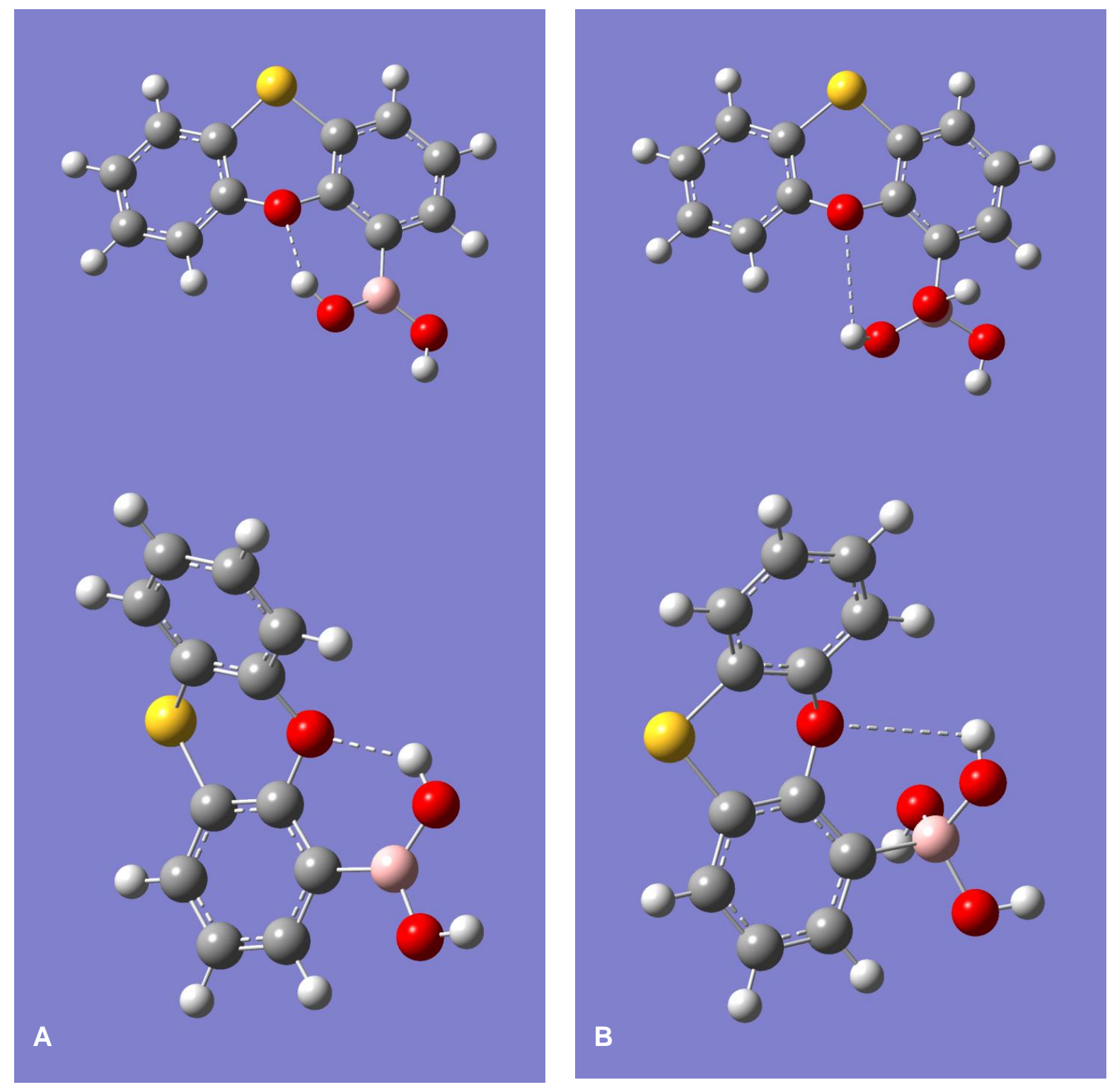

Figure 8. Molecular models for intramolecular hydrogen bond formation of 4-POBA. Distance between the donor species (hydroxyl group of boronic acid) and acceptor species (oxygen of phenoxathiin) is $2.04 \AA$ and $2.84 \AA$ for trigonal planar $(\mathrm{A})$ and tetrahedral $(\mathrm{B})$ hybridizations, respectively. 


\subsection{IONIZATION STATES}

\subsubsection{Isoquinoline-7-Boronic Acid}

To better understand the basic mechanism by which the fluorescence changes occurred, the $\mathrm{p} K_{\mathrm{a}}$ values of 7-IQBA in the absence and presence of various sugars were determined. The fluorescence intensities of boronic acids and their esters were measured at varied $\mathrm{pH}$ values. Titration curves were created from the intensities for each measured $\mathrm{pH}$ value using the emission maximum (maxima). Statistical software was used to fit titration curves. Equation (2a) or (2b) was used and $\mathrm{p} K_{\mathrm{a}}$ values were assigned to coefficient $x 0$ or EC50, respectively. Equation (2a) was used with Sigma Plot 10.0 and (2b) with Sigma Plot 12.0.

(Equation 2a)

$$
\begin{gathered}
f 1=\min +(\max -\min ) /\left(1+\operatorname{abs}(x / E C 50)^{\wedge} \text { Hillslope }\right) \\
\left.f 2=\min +(\max -\min )^{\star}\left(\operatorname{abs}(x / E C 50)^{\wedge} \text { abs }(\text { Hillslope })\right) /\left(1+\operatorname{abs}(x / E C 50)^{\wedge} \text { abs }(\text { Hillslope })\right)\right) \\
f=\operatorname{if}(x<=0, \text { if }(\text { Hillslope }>0, \max , \min ), \text { if }(\text { Hillslope }>0, f 1, f 2))
\end{gathered}
$$

(Equation 2b)

$$
f=y 0+a /(1+\exp (-(x-x 0) / b))
$$


For 7-IQBA, there are two ionizable functional groups: the boronic acid group and the isoquinolinium nitrogen. Therefore, two $\mathrm{p} K_{\mathrm{a}}$ values were expected for 7-IQBA. As shown in Figure 4, the fluorescence intensities of the boronic acids and their presumed esters changed with $\mathrm{pH}$. Such results demonstrate that fluorescent properties are associated with the ionization state of the boronic acids and their corresponding esters. For 7-IQBA, there are two ionizable functional groups: the boronic acid group and the isoquinolinium nitrogen. Therefore, there should be two $\mathrm{p} K_{a}$ values.

Based on literature precedents, ${ }^{15}$ it was reasonable to assume that the ionization steps of 7-IQBA followed route $\mathbf{A}$ in which deprotonation of the isoquinolinium nitrogen precedes the hybridization state change of the boronic acid seen in Scheme $1 .{ }^{15}$ On the other hand, the ionization of esters of 7-IQBA most likely follow route $\mathbf{B}$. Thus, the first ionization step for the esters is the hybridization state change of the boronic ester group, followed by deprotonation of the isoquinolinium nitrogen.

Table 2. Apparent $p K_{\mathrm{a}}$ values of 7-IQBA and 4-POBA.

\begin{tabular}{ccccccc}
\hline & D-fructose & D-glucose & D-mannose & D-sorbitol & D-tagatose & No Sugar \\
\hline 7-IQBA & $4.9,6.9$ & ND $^{[a]}$ & $5.2,7.4$ & $4.1,6.8$ & $5.1,6.9$ & $5.5,7.8$ \\
4-POBA & 6.3 & 6.3 & 6.4 & 6.6 & 6.6 & 8.0 \\
\hline
\end{tabular}

[a] Not determined. 


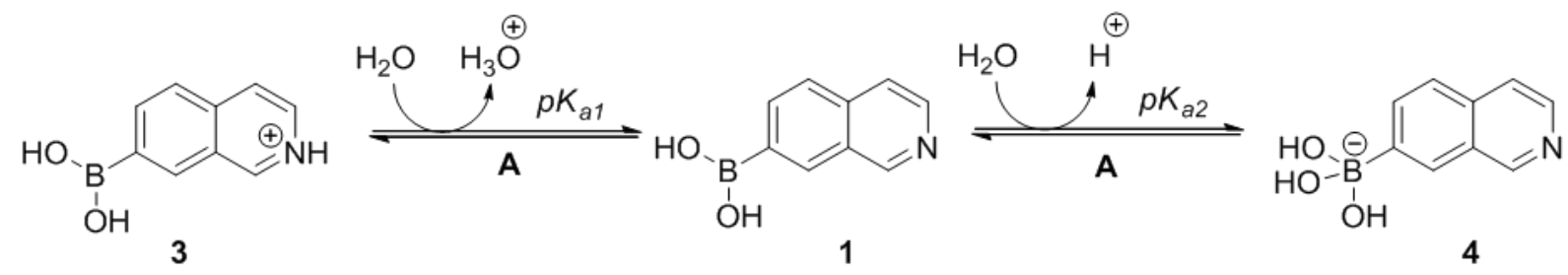

Scheme 1. Proposed ionizations of 7-IQBA in the absence of sugar.

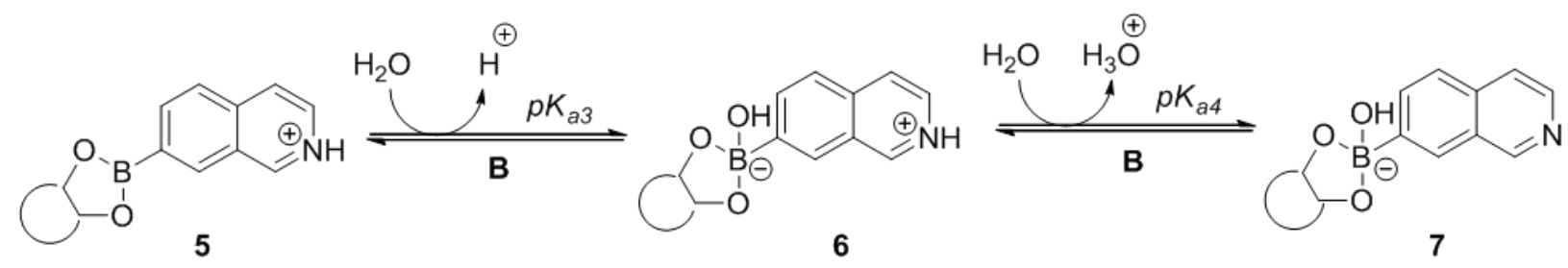

Scheme 2. Proposed ionizations of 7-IQBA in the presence of a sugar. 
It is interesting to note that 7-IQBA and all of its esters have two emission maxima. Therefore, the $\mathrm{pH}$ profiles of the fluorescence intensities were constructed at different wavelengths to obtain the $\mathrm{p} K_{a}$ values listed in Table 2. For 7-IQBA only, it was surprising to find that increasing the $\mathrm{pH}$ induced an $80 \%$ fluorescence intensity decrease at $378 \mathrm{~nm}$ with an apparent $p K_{a}$ value of around 5.5, which was assigned as $p K_{a 1}$ based on comparison with an earlier report. ${ }^{15}$ However, when the $\mathrm{pH}$ profiles of the fluorescence intensities were drawn at 344 $\mathrm{nm}$, two approximate $\mathrm{p} K_{a}$ values could be obtained: 5.5 with $\mathrm{p} K_{a 1}$ and 7.8 with $\mathrm{p} K_{a 2}$. As seen in Figure 4, 7-IQBA itself did not have strong fluorescence $(378 \mathrm{~nm})$ at low $\mathrm{pH}$. At pH 7.4, 7-IQBA existed predominantly in the deprotonated form, which was nearly non-fluorescent.

In the presence of various sugars, the fluorescence intensities of 7-IQBA increased 17-, 13-, 37- and 17-fold at $344 \mathrm{~nm}$, corresponding to the addition of D-fructose, D-mannose, Dsorbitol, and D-tagatose. The apparent $\mathrm{p} K_{\mathrm{a}}$ values were observed as $6.9,7.4,6.8$ and 6.9, respectively. Based on the data obtained from earlier studies, all of these $\mathrm{p} K_{\mathrm{a}}$ values were assigned as $\mathrm{p} K_{a 4 \cdot{ }^{15}}$ For the data collected at $378 \mathrm{~nm}$, the second set of $\mathrm{p} K_{a}$ values were observed as 4.9, 5.2, 4.1 and 5.1 for the esters of D-fructose, D-mannose, D-sorbitol, and Dtagatose, which could be assigned as $\mathrm{p} K_{a 3}$ based on the previous studies. ${ }^{15}$ Such results indicate that the $\mathrm{p} K_{a}$ value of the boronic acid group is higher in the absence of a sugar and lower in the presence of a sugar.

The switching of $\mathrm{p} K_{a}$ also correlated with the strong fluorescence changes at $\mathrm{pH} 7.4$. Thus, it is reasonable to assume that the zwitterionic species (6) is more fluorescent than the boronic ester (5). At pH 7.4, 7-IQBA itself existed predominantly in the neutral trigonalcoordinated boron form (1) since the $\mathrm{p} K_{a}$ of 7-IQBA is 7.8. The D-fructose, D-sorbitol, and Dtagatose esters existed predominantly in the boronate form (7). Since the $p K_{a}$ value of the Dmannose ester of 7-IQBA is 7.4, one would expect to find a mixture of the zwitterionic isoquinolinium boronate (6) and isoquinolinyl boronate forms (7) at $\mathrm{pH} 7.4$. Overall, $\mathbf{1}$ and $\mathbf{4}$ are 
almost non-fluorescent while the boronate ester forms 6 and 7 are fluorescent. Therefore, the binding between 7-IQBA with sugars leads to the fluorescence increase at $\mathrm{pH}$ 7.4. 


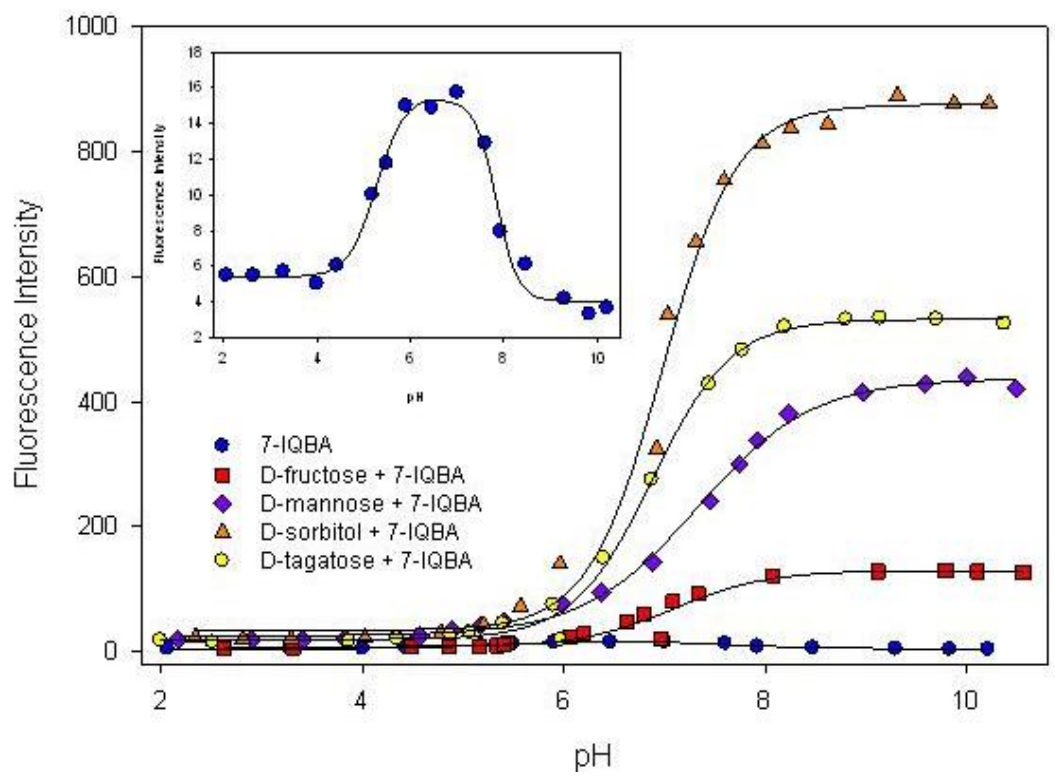

Figure 9. $\mathrm{pH}$ profile of 7-IQBA $(10 \mu \mathrm{M})$ in the absence and presence of various sugars $(0.01 \mathrm{M})$ in phosphate buffer $(0.1 \mathrm{M}): \lambda_{\mathrm{ex}}=272 \mathrm{~nm}$ (7-IQBA only, D-fructose, D-glucose, D-sorbitol, and D-tagatose); $\lambda_{\mathrm{ex}}=271 \mathrm{~nm}$ (D-mannose), $\lambda_{\mathrm{em}}=344 \mathrm{~nm}$. All experiments were performed in duplicate.

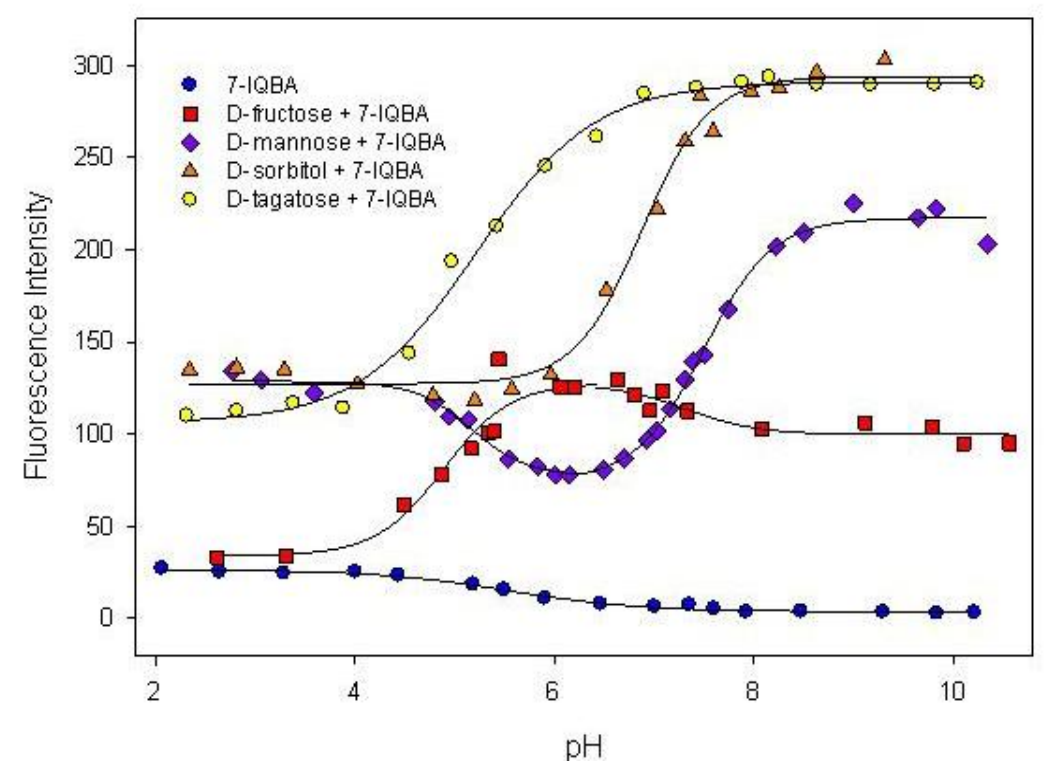

Figure 10. $\mathrm{pH}$ profiles of 7-IQBA $(10 \mu \mathrm{M})$ in the absence and presence of various sugars $(0.01 \mathrm{M})$ in phosphate buffer $(0.1 \mathrm{M}): \lambda_{\mathrm{ex}}=272 \mathrm{~nm}$ (7-IQBA only, D-fructose, D-glucose, D-sorbitol, and D-tagatose); $\lambda_{\mathrm{ex}}=271 \mathrm{~nm}$ (D-mannose), $\lambda_{\mathrm{em}}=378 \mathrm{~nm}$. All experiments were performed in duplicate. 
Table 3. Presumed structures of 7-IQBA and its sugar esters at pH 7.4.

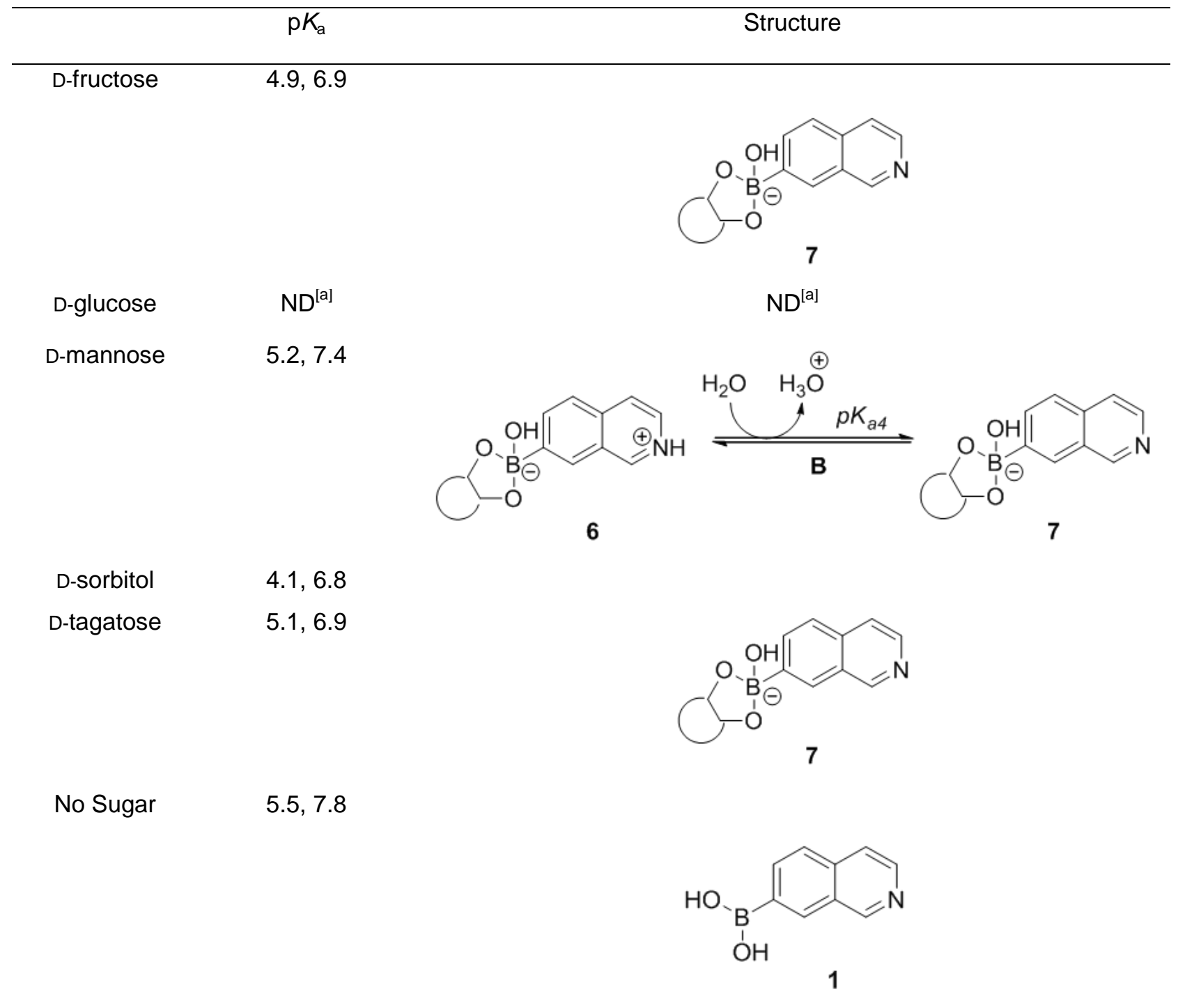

[a] Not determined. 


\subsubsection{Phenoxathiin-4-Boronic Acid}

In the case of 4-POBA, there is only one ionizable group, the boronic acid. It was found that the fluorescence intensity increased 30 -fold when the $\mathrm{pH}$ decreased with an apparent $\mathrm{p} K_{a}$ of 8.0. Such results indicate that the neutral trigonal form (2) is the more fluorescent species. In the presence of sugars, the apparent $p K_{a}$ values were 6.3 (D-fructose ester and D-glucose ester), 6.4 (D-mannose ester), and 6.6 (D-sorbitol ester and D-tagatose ester). Similar to 4POBA alone, the trigonal neutral ester form (9) is the more fluorescent species, while the tetrahedral ester form (10) is mostly non-fluorescent (Scheme 2). At pH 7.4, 4-POBA exists in the trigonal form (2) and 4-POBA ester exists predominantly in the tetrahedral form (10). The boronic acid form of 4-POBA (2) is fluorescent and the boronate form (10) is non-fluorescent. Therefore, the binding between 4-POBA with sugars leads to a decrease in fluorescence intensity. 

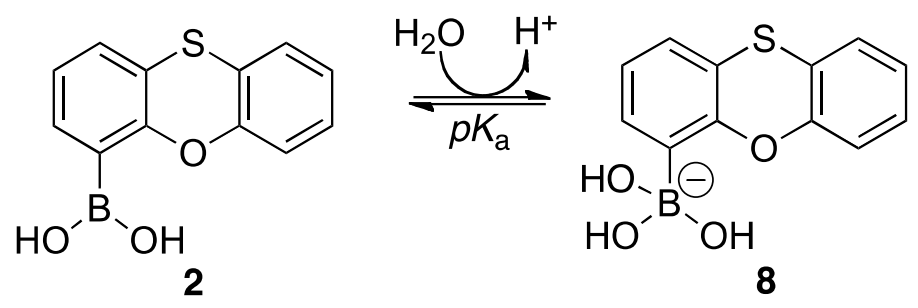

Scheme 3. Proposed ionization of 4-POBA in the absence of sugar.
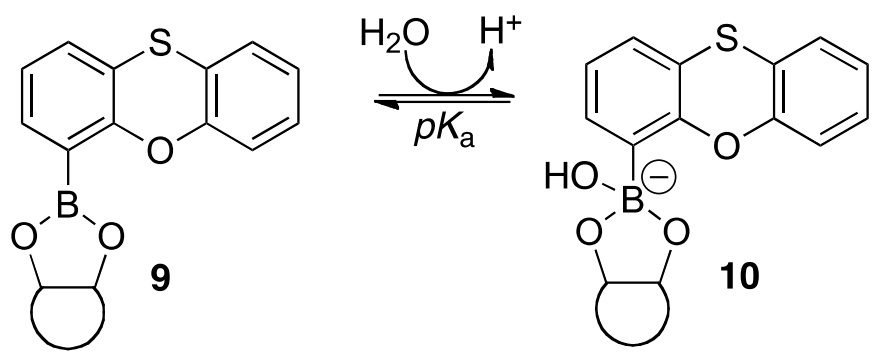

Scheme 4. Proposed ionization of 4-POBA in the presence of sugar. 


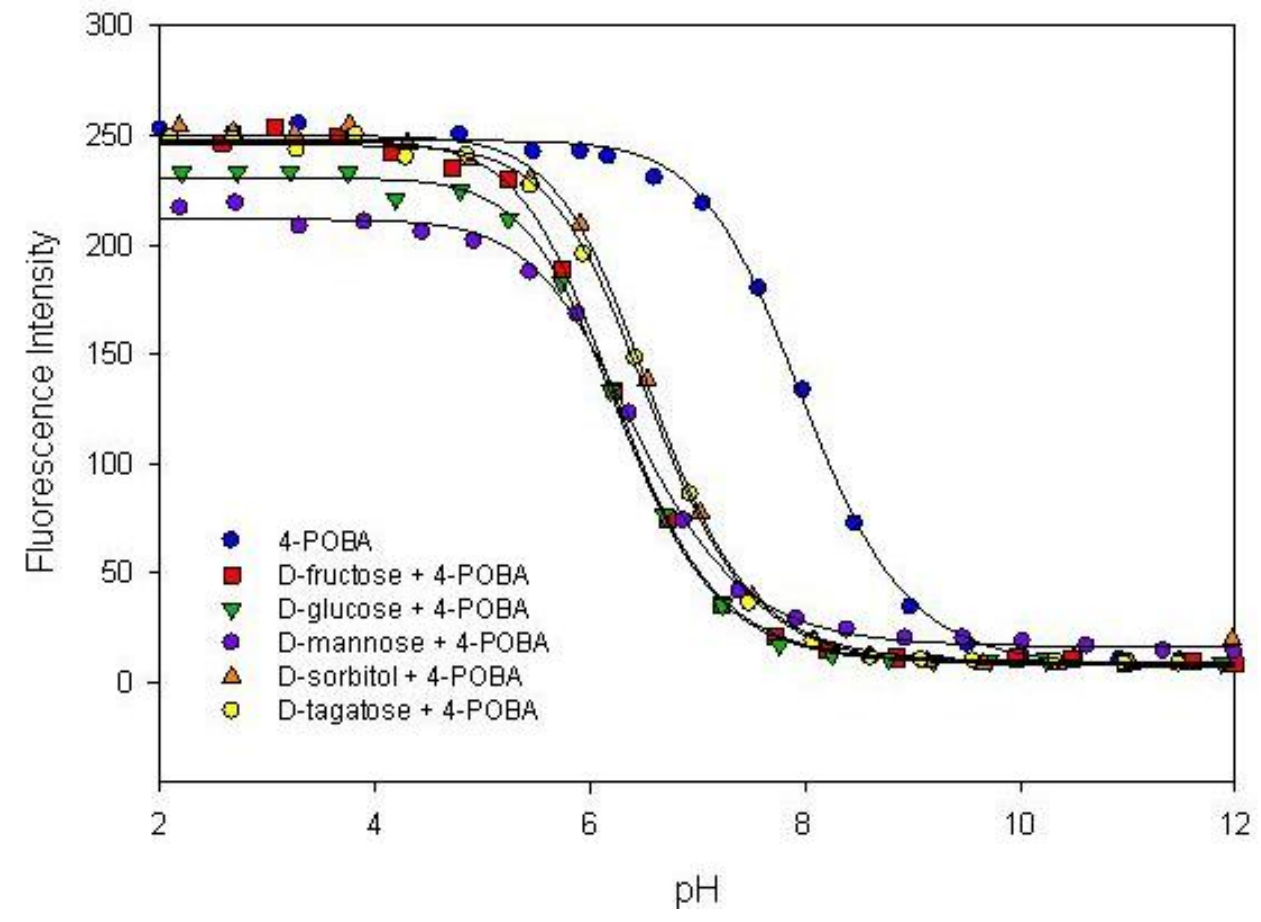

Figure 11. $\mathrm{pH}$ profiles of 4-POBA $(10 \mu \mathrm{M})$ in the absence and presence of various sugars $(0.01 \mathrm{M})$ in phosphate buffer $(0.1 \mathrm{M}): \lambda_{\mathrm{ex}}=299 \mathrm{~nm}, \lambda_{\mathrm{em}}=430 \mathrm{~nm}$. All experiments were performed in duplicate. 
Table 4. Presumed structures of 4-POBA and its sugar esters at pH 7.4.

\begin{tabular}{|c|c|c|}
\hline & $p K_{a}$ & Structure \\
\hline D-fructose & 6.3 & \\
\hline D-glucose & 6.3 & \\
\hline D-mannose & 6.4 & \\
\hline D-sorbitol & 6.6 & \\
\hline D-tagatose & 6.6 & 10 \\
\hline No Sugar & 8.0 & \\
\hline
\end{tabular}




\subsection{FLUORESCENCE QUANTUM YIELD $\left(\Phi_{\mathrm{F}}\right)$}

A fluorescence quantum yield test is a measure of how efficient the fluorescence from a species is. By definition, the fluorescence quantum yield is the number of photons emitted over the number of photons absorbed. Quantum yield can be calculated from a photon counter using equation (3). Our laboratory does not have an instrument of this nature so relative fluorescence quantum yields were determined using equation (4). We compared the unknown boronic acids and their esters to a reference. We used 8-IQBA as the reference because it has a known quantum yield $(2.2 \%)$ and an excitation wavelength maximum $(271 \mathrm{~nm})$ near those of 7 -IQBA and 4-POBA. From the known quantum yield of 8-IQBA, we varied the concentrations of 8-IQBA and measured the absorbance and fluorescence. A curve of integrated fluorescence peak area over absorbance intensity (Figure 12) was created to obtain the slope. We repeated this for 7 IQBA by obtaining the absorbance intensities and the fluorescence peak areas.

(Equation 3)

$$
\Phi_{F}=\text { photons emitted/photons absorbed }
$$

(Equation 4)

$$
\Phi_{F}=Q=Q_{R}\left(\frac{I}{I_{R}}\right) \cdot\left(\frac{O D_{R}}{O D}\right)
$$

Using the slopes and the known quantum yield of 8-IQBA, we used equation (4) to set up a ratio of the known values to determine the quantum yield of 7-IQBA. This same procedure was duplicated for free boronic acids and their sugar-esters. 


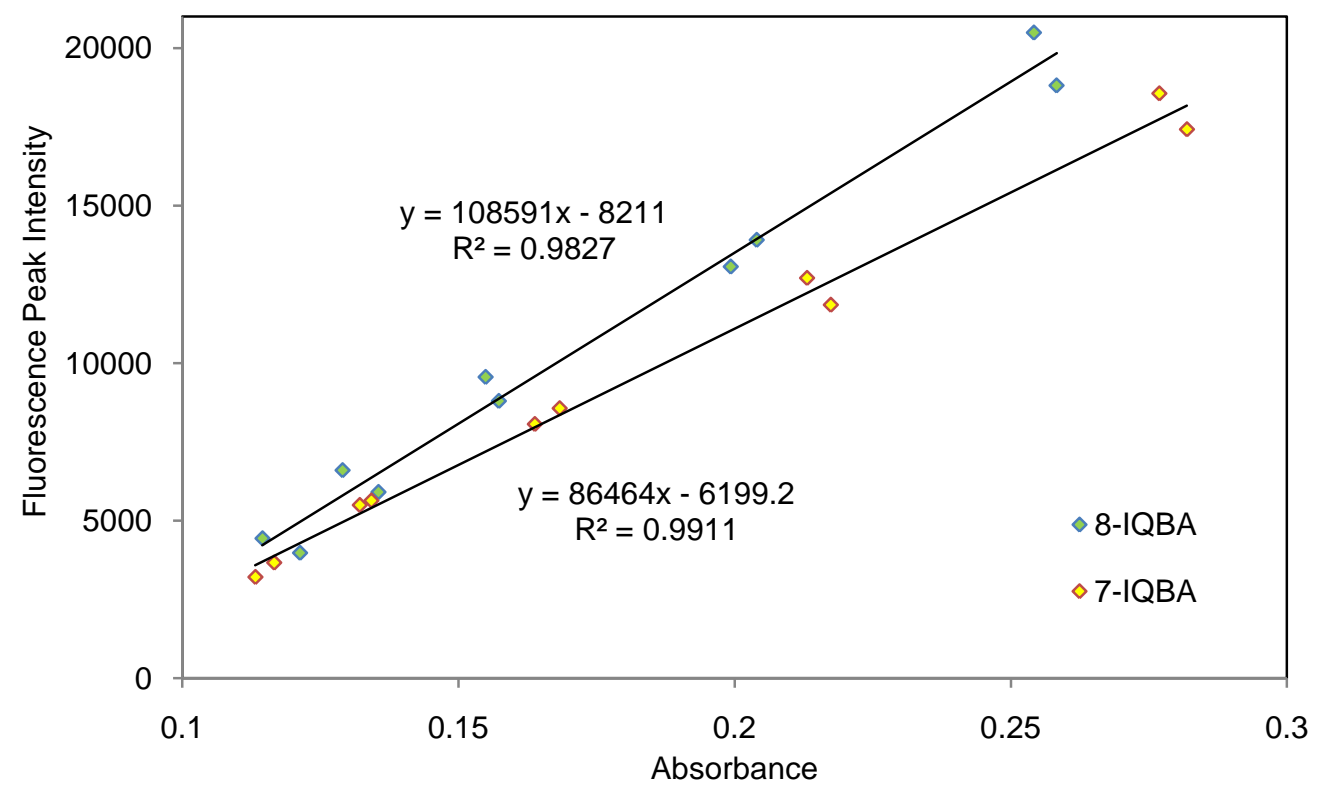

Figure 12. Fluorescence quantum yield slope determination of 8-IQBA and 7-IQBA. Fluorescence quantum yield slopes of 8 -IQBA and 7-IQBA $(1 / 3$ dilutions of $10 \mu \mathrm{M})$ in phosphate buffer $(0.1 \mathrm{M})$ at $\mathrm{pH}$ 7.4: $\lambda_{\mathrm{ex}}=271 \mathrm{~nm}, \lambda_{\mathrm{em}}$ range $=315-421 \mathrm{~nm}$. Experiments were duplicated. 
The results for the fluorescence quantum yields are shown in Table 2. The quantum yields for 7-IQBA in the absence and presence of saccharide followed the trend D-fructose ester > D-tagatose ester > D-sorbitol ester > D-mannose ester > D-glucose ester > 7-IQBA only. Sugar complexes had higher quantum yields than 7-IQBA itself. For example, the quantum yield for the D-fructose ester of 7-IQBA was $48 \%$ while 7 -IQBA alone was only $2 \%$, a 24 -fold difference. D-Tagatose ester and D-sorbitol ester also had high quantum yields of $34 \%$ and $28 \%$, respectively. D-Mannose ester showed a lower quantum yield of $8 \%$, but still over 4 -fold higher than 7-IQBA itself.

Table 5. Fluorescence quantum yields $\left(\Phi_{\mathrm{F}}\right)$ of 7-IQBA and 4-POBA.

\begin{tabular}{ccccccc}
\hline \multicolumn{7}{c}{$\Phi_{\mathrm{F}}(\%)$} \\
\hline 7-IQBA & D-fructose & D-glucose & D-mannose & D-sorbitol & D-tagatose & No Sugar \\
4-POBA & $48 \pm 0.55$ & $\mathrm{ND}^{\text {[a] }}$ & $7.8 \pm 0.06$ & $28 \pm 0.24$ & $34 \pm 1.2$ & $1.7 \pm 0.13$ \\
& $0.72 \pm 0.05$ & $0.72 \pm 0.05$ & $0.55 \pm 0.05$ & $0.86 \pm 0.06$ & $0.99 \pm 0.07$ & $8.0 \pm 0.16$ \\
\hline
\end{tabular}

[a] Not determined.

The quantum yield for 4-POBA displayed a very different tendency compared to 7-IQBA. The boronic acid itself had a much higher quantum yield than any of the sugar ester forms. For instance, the quantum yield for 4-POBA was $8 \%$, while quantum yields for boronates fell below $1 \%$, which is consistent with the binding study as shown in Figure 6 and 7 where the fluorescence intensities decreased upon binding to sugar. For instance, D-tagatose ester $(0.99 \%)$ showed the smallest fluorescence intensity decrease of 8 -fold and 14 to 20 -fold decreases from D-fructose ester (0.72\%), D-glucose ester $(0.72 \%)$, D-mannose ester $(0.55 \%)$, and D-sorbitol ester (0.86\%). The dominant fluorescent species was 4-POBA alone. 
Environmental factors (e.g. solvent, buffer, ions) may affect fluorescence and factors such as species aggregation may influence the quantum yield. For instance, at higher concentrations of 8-IQBA, we observed the fluorescence intensity to reach a maximum, which was likely due to species aggregation in solution. As seen in Figure 13, the maximum fluorescence intensity decreased upon reaching a certain concentration (around $12 \mu \mathrm{M}$ ). Therefore, low concentrations of boronic acids $(10 \mu \mathrm{M})$ were used. In buffered solutions, there are often issues with buffer interactions. The presence of ions may have a quenching effect on fluorescence. However, phosphate ions with boronic acid have no effect on fluorescence intensity which is why phosphate buffer is commonly used..$^{7 d}$ 

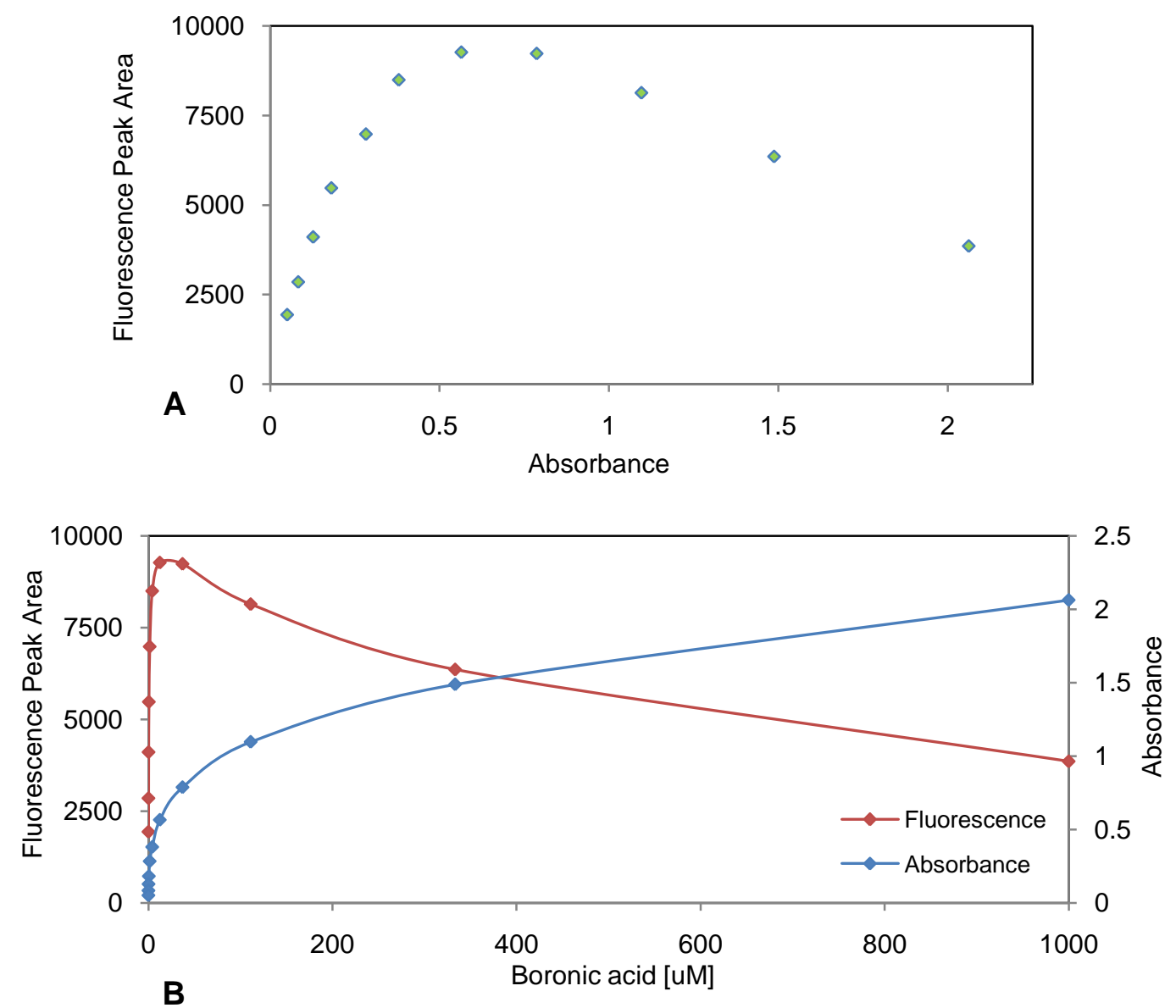

Figure 13. Evidence of species aggregation for 8-IQBA. Integrated fluorescence area versus absorbance intensities of 8-IQBA. B) Fluorescence peak area and absorbance intensity versus 8IQBA concentrations $(16 \mathrm{pM}-1 \mathrm{mM}) . \lambda_{\mathrm{ex}}=271 \mathrm{~nm}, \lambda_{\mathrm{em}}$ range $=315-421 \mathrm{~nm}$. 


\section{CONCLUSIONS}

Isoquinoline-7-boronic acid and phenoxathiin-4-boronic acid were examined to determine their potential use as scaffolds in chemosensor design. Several properties of these two arylboronic acids were studied under physiological conditions, which included the apparent binding affinity, proposed ionization schemes, and fluorescence quantum yields. Binding of 7IQBA and 4-POBA with several monosaccharides was reported using micromolar concentrations of boronic acid. Both boronic acids showed large changes in fluorescence intensity upon the addition of sugar. Using this data, sensors can be designed accordingly, depending on desired characteristics for the recognition of a particular biomarker. These results indicate that 7-IQBA may be more useful than 4-POBA in the design and synthesis of boronic acid scaffolds for sensing carbohydrates in biological systems.

\section{EXPERIMENTAL}

Absorbance spectra were obtained using Shimadzu PharmaSpec UV-1700 UV-Visible Spectrophotometer and UVProbe Software. Fluorescence emission spectra were obtained using Shimadzu RF-5301PC Spectrofluorophotometer and RF530XPC Software. Quartz crystal cuvettes were used for absorbance and fluorescence experiments. All $\mathrm{pH}$ values were determined using Fisher Scientific Accumet Research AR10 pH Meter. Water used for buffer solutions was purified using Thermo Scientific Barnstead Nanopure filtration system. All $p K_{a}$ values were calculated using Systat Software Inc. SigmaPlot 12.

Buffer was prepared with $\mathrm{Na}_{2} \mathrm{HPO}_{4}(0.077 \mathrm{~mol})$ and $\mathrm{NaH}_{2} \mathrm{PO}_{4}(0.023 \mathrm{~mol})$ in $\mathrm{dH}_{2} \mathrm{O}(1 \mathrm{~L})$, and adjusted to $\mathrm{pH}$ 7.4. Isoquinoline-7-boronic acid and phenoxathiin-4-boronic acid were commercially available from Frontier Scientific. Boronic acids were dissolved in $\mathrm{MeOH}$ to $0.01 \mathrm{M}$ and diluted to $10 \mu \mathrm{M}$ using $0.1 \mathrm{M}$ phosphate buffer at $\mathrm{pH}$ 7.4. Sugars were available though 
Fisher Scientific and Sigma-Aldrich and prepared in $0.1 \mathrm{M}$ phosphate buffer, pH 7.4. Excitation wavelengths were determined by absorbance spectra.

For binding affinities, standard solutions of D-glucose (1.5 M), D-mannose (0.5 M), and D-fructose, D-sorbitol and D-tagatose $(10 \mathrm{mM})$ were prepared with boronic acid $(10 \mu \mathrm{M})$ in phosphate buffer $(0.1 \mathrm{M}), \mathrm{pH}$ 7.4. Experiments were performed in triplicate. To determine $\mathrm{p} K_{\mathrm{a}}$ values, saccharide solutions $(0.01 \mathrm{M})$ were prepared in boronic acid $(10 \mu \mathrm{M})$ in phosphate buffer $(0.01 \mathrm{M}), \mathrm{pH}$ 7.4. The $\mathrm{pH}$ was adjusted from 2 to 12 using concentrated $\mathrm{HCl}$ and saturated $\mathrm{NaOH}$, in keeping with consistent volumes $(40 \mathrm{~mL})$ and concentrations. Experiments were duplicated. For quantum yield studies, solutions of boronic acid $(10 \mu \mathrm{M})$ with and without sugars were prepared ( $0.03 \mathrm{M}$ for $\mathrm{D}$-mannose, $0.01 \mathrm{M}$ for all others) in phosphate buffer $(0.01 \mathrm{M}), \mathrm{pH}$ 7.4. One-third, serial dilutions of boronic acid $(10 \mu \mathrm{M})$ were made to obtain absorbance and fluorescence emission spectral data. All experiments were duplicated. 
1. Righettoni, M.; Tricoli, A.; Pratsinis, S. E., Si:WO(3) Sensors for highly selective detection of acetone for easy diagnosis of diabetes by breath analysis. Anal Chem 82 (9), 3581 7. 2. $\quad$ DeGrado, T. R.; Baldwin, S. W.; Wang, S. Y.; Orr, M. D.; Liao, R. P.; Friedman, H. S.; Reiman, R.; Price, D. T.; Coleman, R. E., Synthesis and evaluation of F-18-labeled choline analogs as oncologic PET tracers. J Nucl Med 2001, 42 (12), 1805-1814.

3. Colombo, G.; Morra, G.; Genoni, A.; Neves, M. A. C.; Merz, K. M., Molecular Recognition and Drug-Lead Identification: What Can Molecular Simulations Tell Us? Current Medicinal Chemistry 2010, $17(1), 25-41$.

4. (a) Fischer, A.; Hacein-Bey-Abina, S.; Cavazzana-Calvo, M., Gene therapy of metabolic diseases. Journal of Inherited Metabolic Disease 2006, 29 (2-3), 409-412; (b) Kay, M. A., Stateof-the-art gene-based therapies: the road ahead. Nat Rev Genet 12 (5), 316-28; (c) Stangl, M. J.; Beuers, U.; Schauer, R.; Lang, T.; Gerbes, A.; Briegel, J.; Da Silva, L.; Schildberg, F. W.; Rau, H. G., Allogenic liver transplantation - a form of "gene therapy" in metabolic diseases. Munich results and review. Chirurg 2000, 71 (7), 808-819; (d) Joshi, A.; Garg, H.; Ablan, S.; Freed, E. O.; Nagashima, K.; Manjunath, N.; Shankar, P., Targeting the HIV entry, assembly and release pathways for anti-HIV gene therapy. Virology 2011, 415 (2), 95-106.

5. (a) Suzuki, A.; Miyaura, N., New Synthetic Reactions of Organoboron Compounds by Transition-Metal Catalysts. Journal of Synthetic Organic Chemistry Japan 1993, 51 (11), 10431052; (b) Miyaura, N.; Ishiyama, T.; Kizaki, H.; Hayashi, T.; Suzuki, A., Palladium-catalyzed carbonylative cross-coupling reaction of arylboronic acids with aryl electrophiles: Synthesis of biaryl ketones. J Org Chem 1998, 63 (14), 4726-4731; (c) Voskoboynikov, A. Z.; Izmer, V. V.; Lebedev, A. Y.; Nikulin, M. V.; Ryabov, A. N.; Asachenko, A. F.; Lygin, A. V.; Sorokin, D. A., Palladium-catalyzed pathways to aryl-substituted indenes: Efficient synthesis of ligands and the respective ansa-zirconocenes. Organometallics 2006, 25 (5), 1217-1229; (d) Miyaura, N.; Ishikawa, M.; Suzuki, A., Stereoselective Synthesis of Exocyclic Alkenes Via HydroborationCross-Coupling Sequence. Tetrahedron Letters 1992, 33 (18), 2571-2574.

6. (a) Vakulenko, S. B.; Toth, M.; Chow, J. W.; Mobashery, S., Source of Phosphate in the Enzymic Reaction as a Point of Distinction among Aminoglycoside 2 "-Phosphotransferases. Journal of Biological Chemistry 2009, 284 (11), 6690-6696; (b) Kanekiyo, Y.; Naganawa, R.; Tao, H., Fluorescence detection of ATP based on the ATP-mediated aggregation of pyreneappended boronic acid on a polycation. Chem Commun (Camb) 2004, (8), 1006-7; (c) Kim, H. N.; Moon, J. H.; Kim, S. K.; Kwon, J. Y.; Jang, Y. J.; Lee, J. Y.; Yoon, J., Fluorescent sensing of triphosphate nucleotides via anthracene derivatives. J Org Chem 76 (10), 3805-11.

7. (a) Halo, T. L.; Appelbaum, J.; Hobert, E. M.; Balkin, D. M.; Schepartz, A., Selective recognition of protein tetraserine motifs with a cell-permeable, pro-fluorescent bis-boronic acid. J Am Chem Soc 2009, 131 (2), 438-9; (b) Sauvage, E.; Zervosen, A.; Herman, R.; Kerff, F.; Herman, A.; Bouillez, A.; Prati, F.; Pratt, R. F.; Frere, J. M.; Joris, B.; Luxen, A.; Charlier, P., Unexpected Tricovalent Binding Mode of Boronic Acids within the Active Site of a PenicillinBinding Protein. J Am Chem Soc 2011, 133 (28), 10839-10848; (c) Lim, S.; Escobedo, J. O.; Lowry, M.; Strongin, R. M., Detecting specific saccharides via a single indicator. Chemical Communications 47 (29), 8295-8297.

8. Zhao, J. Z.; Chi, L.; James, T. D., Chiral mono boronic acid as fluorescent enantioselective sensor for mono alpha-hydroxyl carboxylic acids. J Org Chem 2008, 73 (12), 4684-4687.

9. $\quad$ Zhang, D. Q.; Tan, W.; Wang, Z.; Liu, C. M.; Zhu, D. B., 4-(N,Ndimethylamine)benzonitrile (DMABN) derivatives with boronic acid and boronate groups: new 
fluorescent sensors for saccharides and fluoride ion. Journal of Materials Chemistry 2007, 17 (19), 1964-1968.

10. (a) James, T. D.; Sandanayake, K. R. A. S.; Shinkai, S., Novel Photoinduced ElectronTransfer Sensor for Saccharides Based on the Interaction of Boronic Acid and Amine. Journal of the Chemical Society-Chemical Communications 1994, (4), 477-478; (b) James, T. D.; Cooper, C. R., Selective fluorescence signalling of saccharides in their furanose form. Chemistry Letters 1998, (9), 883-884; (c) Schanze, K. S.; DiCesare, N.; Pinto, M. R.; Lakowicz, J. R., Saccharide detection based on the amplified fluorescence quenching of a water-soluble poly(phenylene ethynylene) by a boronic acid functionalized benzyl viologen derivative. Langmuir 2002, 18 (21), 7785-7787; (d) DiCesare, N.; Lakowicz, J. R., Chalcone-analogue fluorescent prfobes for saccharides signaling using the boronic acid group. Tetrahedron Letters 2002, 43 (14), 26152618; (e) Ward, C. J.; Patel, P.; James, T. D., Boronic acid appended azo dyes-colour sensors for saccharides. Journal of the Chemical Society, Perkin Transactions 1 2002, (4), 462-470; (f) Ni, W.; Fang, H.; Springsteen, G.; Wang, B., The Design of Boronic Acid Spectroscopic Reporter Compounds by Taking Advantage of the pKa-Lowering Effect of Diol Binding:â€\%o Nitrophenol-Based Color Reporters for Diols. The Journal of Organic Chemistry 2004, 69 (6), 1999-2007; (g) Cordes, D. B.; Miller, A.; Gamsey, S.; Sharrett, Z.; Thoniyot, P.; Wessling, R.; Singaram, B., Optical glucose detection across the visible spectrum using anionic fluorescent dyes and a viologen quencher in a two-component saccharide sensing system. Organic \& Biomolecular Chemistry 2005, 3 (9), 1708-1713; (h) Mulla, K.; Dongare, P.; Zhou, N.; Chen, G.; Thompson, D. W.; Zhao, Y., Highly sensitive detection of saccharides under physiological conditions with click synthesized boronic acid-oligomer fluorophores. Org Biomol Chem 9 (5), 1332-6.

11. Kerr, I. D.; Jones, P. M.; George, A. M., Multidrug efflux pumps: The structures of prokaryotic ATP-binding cassette transporter efflux pumps and implications for our understanding of eukaryotic P-glycoproteins and homologues. Febs Journal 2010, 277 (3), 550 563.

12. (a) Endo, T., Finding of O-mannosyl glycan in mammals and congenital muscular dystrophies due to glycosylation defects. Yakugaku Zasshi-Journal of the Pharmaceutical Society of Japan 2003, 123 (10), 825-835; (b) Rudd, P. M.; Dwek, R. A., Glycosylation: Heterogeneity and the 3D structure of proteins. Critical Reviews in Biochemistry and Molecular Biology 1997, 32 (1), 1-100.

13. Iwasaki, N.; Matsuhashi, T.; Nakagawa, H.; Hato, M.; Kurogochi, M.; Majima, T.; Minami, A.; Nishimura, S. I., Alteration of $\mathrm{N}$-glycans related to articular cartilage deterioration after anterior cruciate ligament transection in rabbits. Osteoarthritis and Cartilage 2008, 16 (7), 772 778.

14. (a) Dennis, J. W.; Granovsky, M.; Warren, C. E., Glycoprotein glycosylation and cancer progression. Biochimica Et Biophysica Acta-General Subjects 1999, 1473 (1), 21-34; (b) Hirohashi, S.; Kanai, Y., Cell adhesion system and human cancer morphogenesis. Cancer Science 2003, 94 (7), 575-581.

15. Wang, B. H.; Cheng, Y. F.; Ni, N. T.; Yang, W. Q., A New Class of Fluorescent Boronic Acids That Have Extraordinarily High Affinities for Diols in Aqueous Solution at Physiological pH. Chemistry-a European Journal 2010, 16 (45), 13528-13538.

16. Experiments performed by Mr. Yingji Wang. 


\section{APPENDICES}

\section{APPEnDIX A - Apparent Association Constants $\left(K_{\mathrm{a}}\right)$}

Isoquinoline-7-boronic acid + D-Fructose

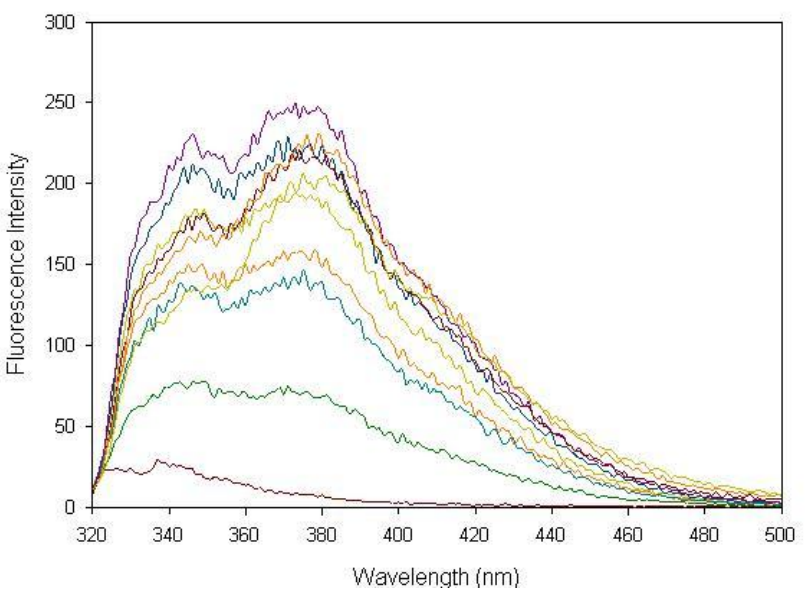

Isoquionoline-7-boronic acid + D-Fructose ${ }^{[16]}$

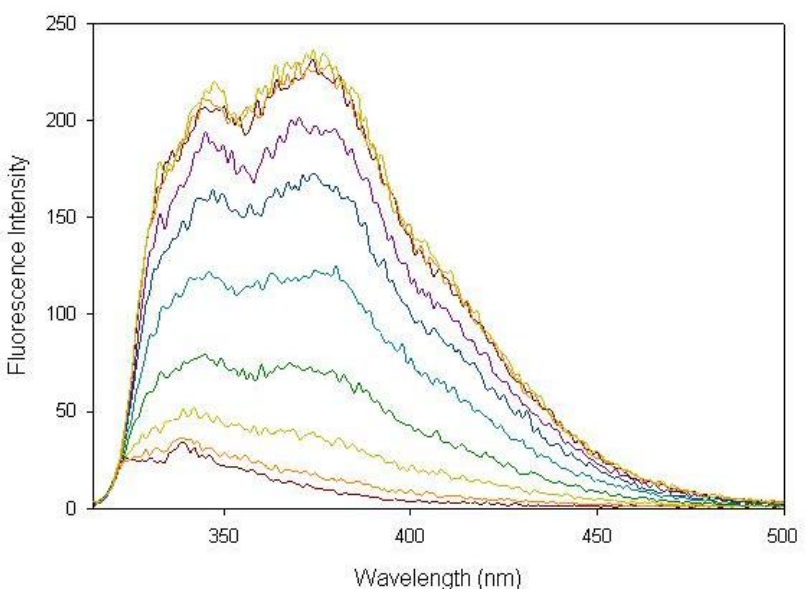

Isoquinoline-7-boronic acid + D-Fructose ${ }^{[16]}$

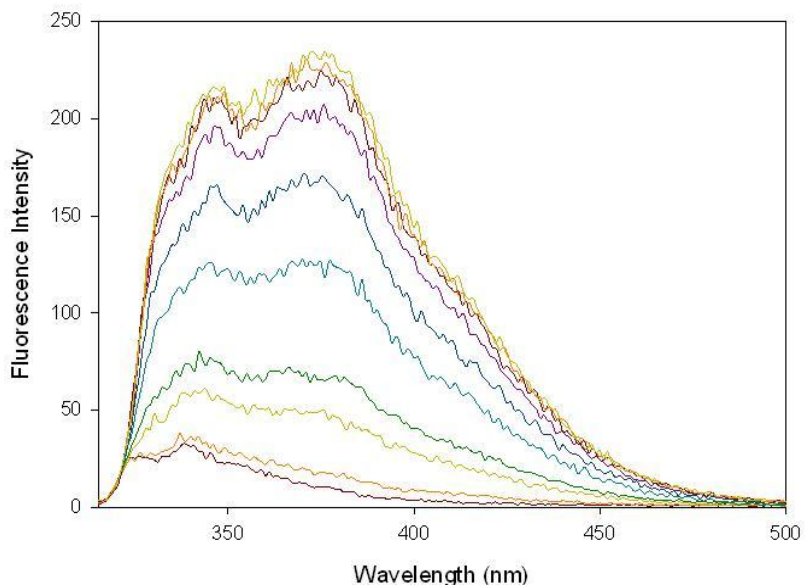

Figure A1. Fluorescent spectral changes of 7-IQBA $\left(1 \times 10^{-5} \mathrm{M}\right)$ upon addition of D-fructose in phosphate buffer $(0.1 \mathrm{M})$ at $\mathrm{pH} 7.4: \lambda_{\mathrm{ex}}=280 \mathrm{~nm}, \lambda_{\mathrm{em}}=370 \mathrm{~nm}$. 
Isoquinoline-7-boronic acid + D-Glucose

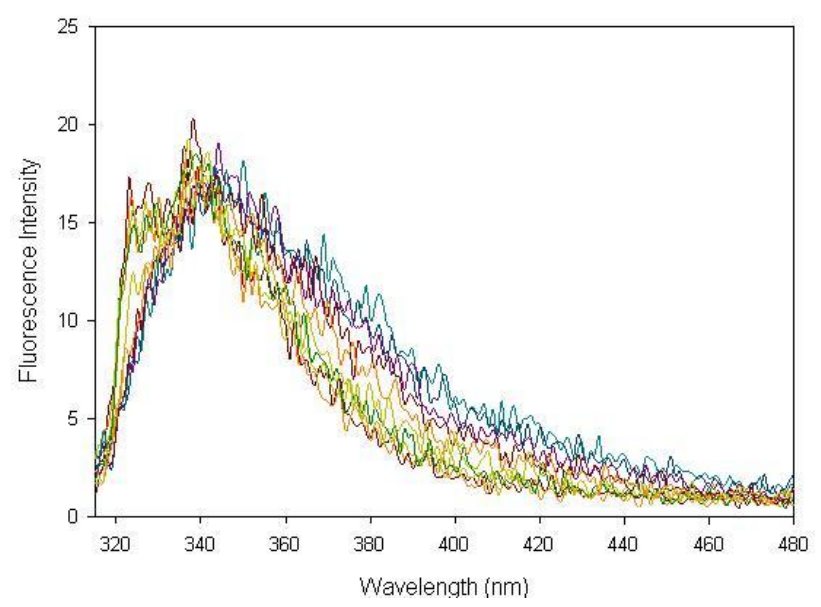

Isoquinoline-7-boronic acid + D-Glucose ${ }^{[16]}$

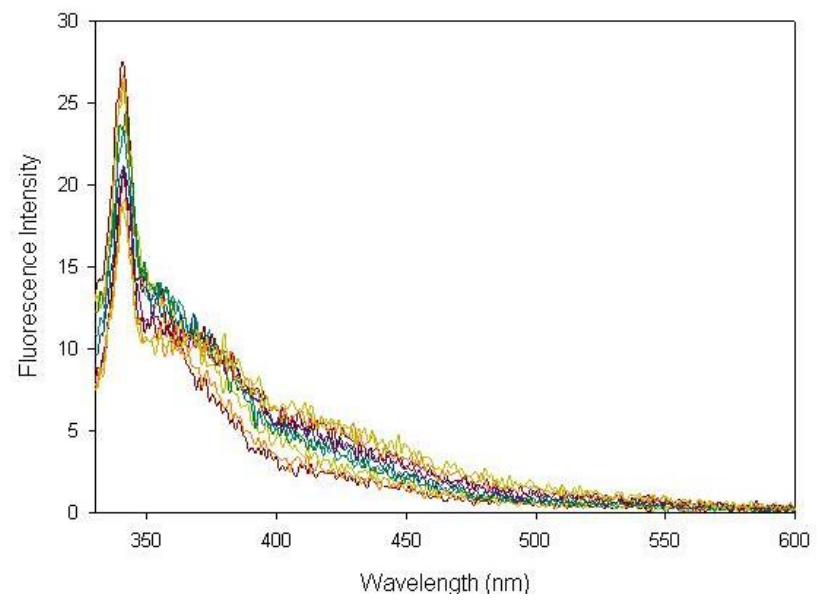

Isoquinoline-7-boronic acid + D-Glucose

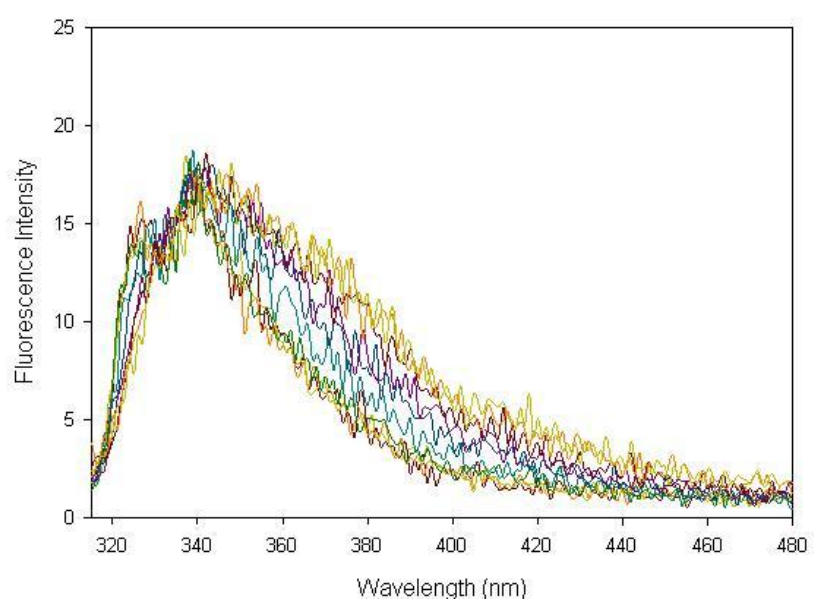

Figure A2. Fluorescent spectral changes of 7-IQBA $\left(1 \times 10^{-5} \mathrm{M}\right)$ upon addition of D-glucose in phosphate buffer $(0.1 \mathrm{M})$ at $\mathrm{pH}$ 7.4: $\lambda_{\mathrm{ex}}=\mathrm{nm}, \lambda_{\mathrm{em}} 344=\mathrm{nm}$. 
Isoquinoline-7-boronic acid + D-Mannose

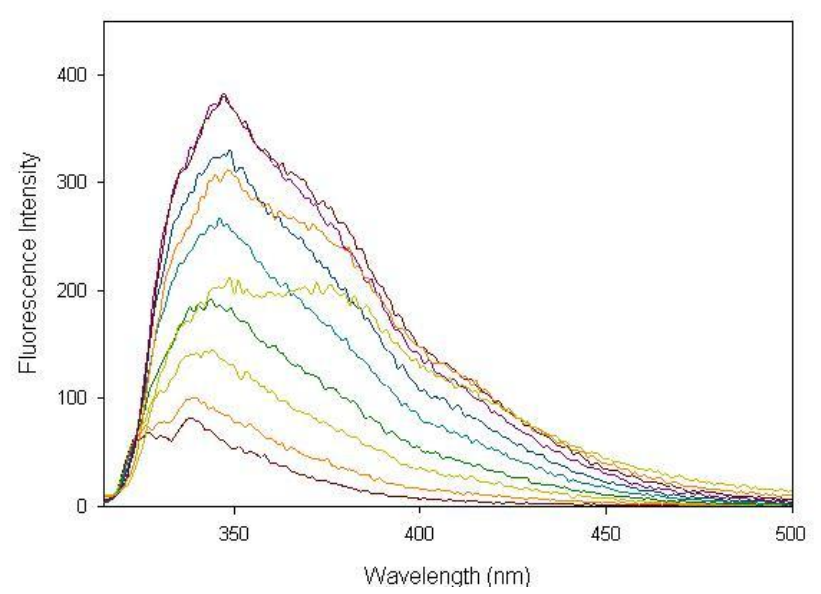

Isoquinoline-7-boronic acid + D-Mannose

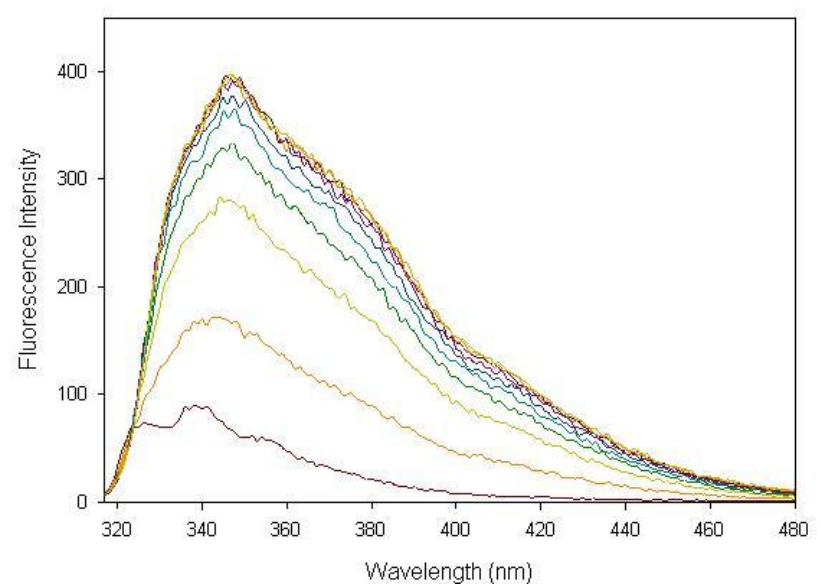

Isoquinoline-7-boronic acid + D-Mannose

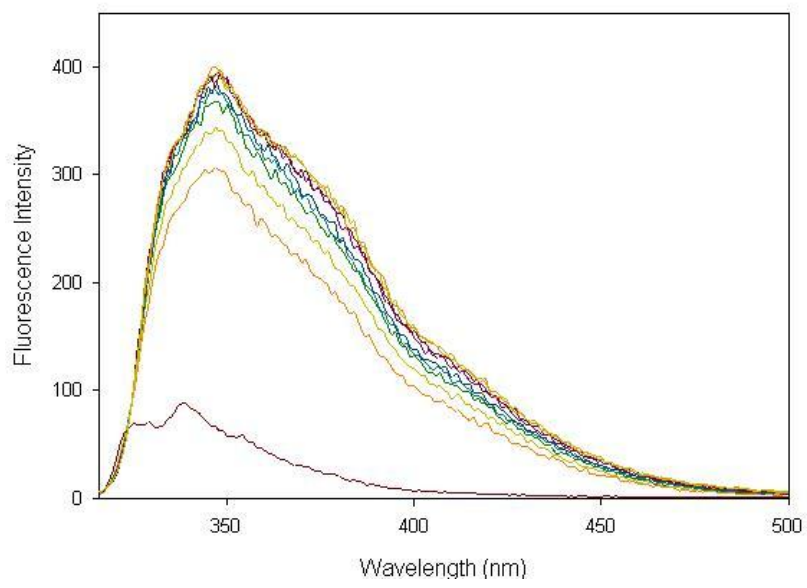

Figure A3. Fluorescent spectral changes of 7-IQBA $\left(1 \times 10^{-5} \mathrm{M}\right)$ upon addition of D-mannose in phosphate buffer $(0.1 \mathrm{M})$ at $\mathrm{pH} 7.4: \lambda_{\mathrm{ex}}=272 \mathrm{~nm}, \lambda_{\mathrm{em}}=347 \mathrm{~nm}$. 
Isoquinoline-7-boronic acid + D-Sorbitol ${ }^{[16]}$

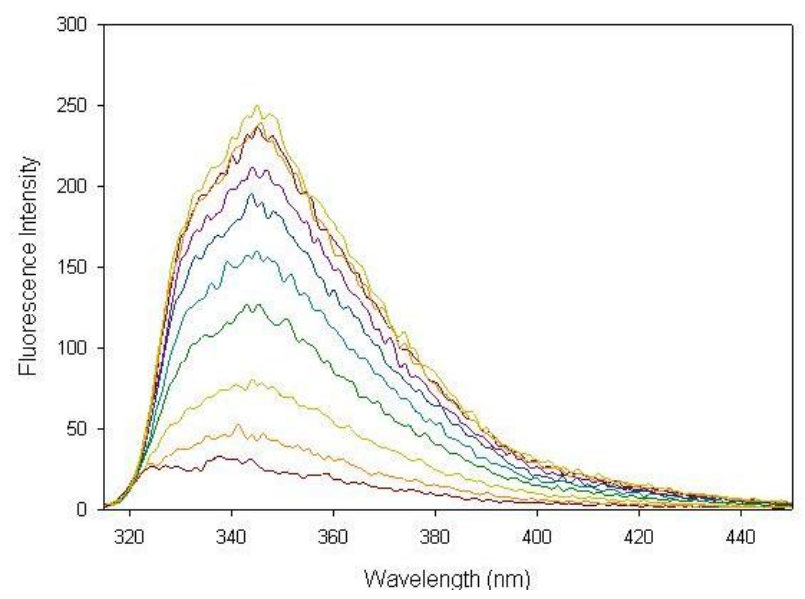

Isoquinoline-7-boronic acid + D-Sorbitol ${ }^{[16]}$

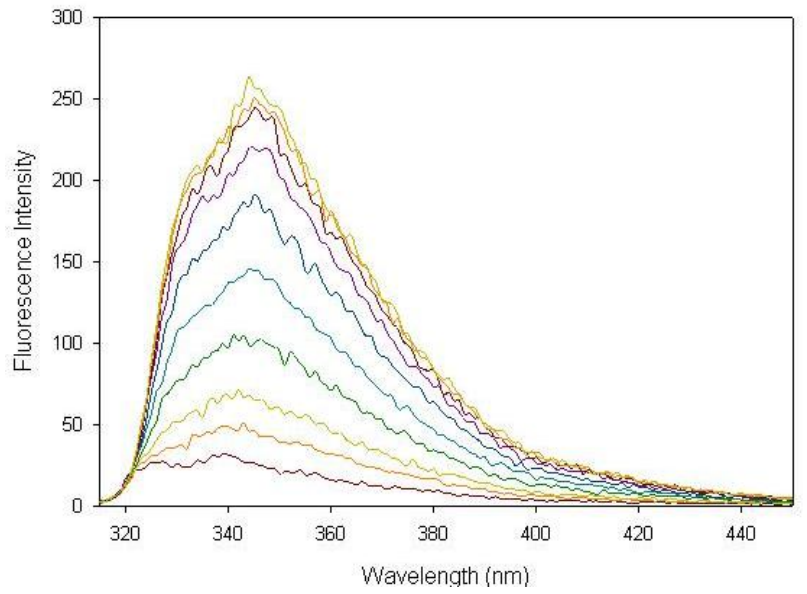

Figure A4. Fluorescent spectral changes of 7-IQBA $\left(1 \times 10^{-5} \mathrm{M}\right)$ upon addition of D-sorbitol in phosphate buffer $(0.1 \mathrm{M})$ at $\mathrm{pH} 7.4: \lambda_{\mathrm{ex}}=280 \mathrm{~nm}, \lambda_{\mathrm{em}}=344 \mathrm{~nm}$. 
Isoquinoline-7-boronic acid + D-Tagatose

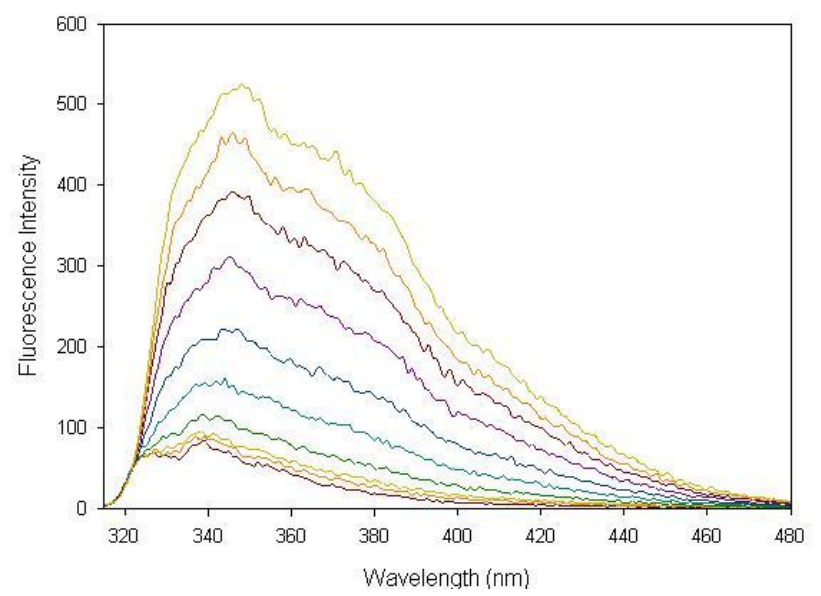

Isoquinoline-7-boronic acid + D-Tagatose

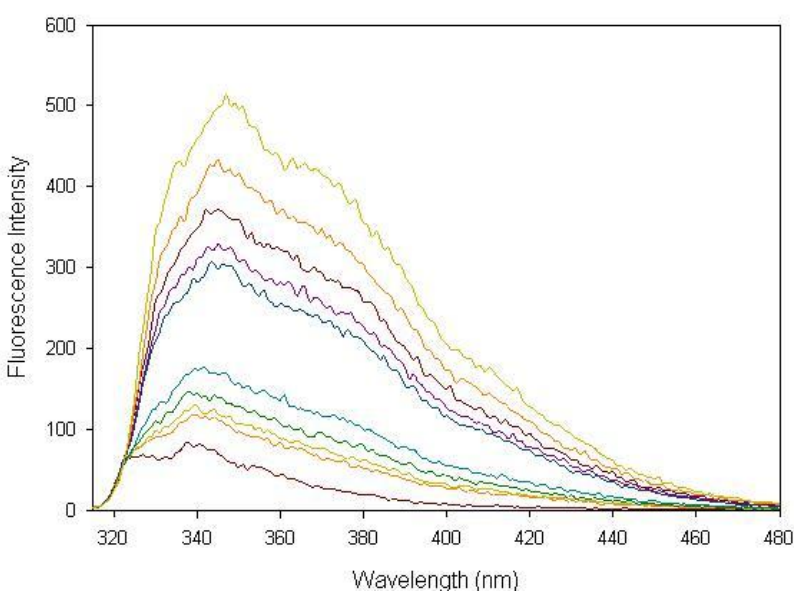

Isoquinoline-7-boronic acid + D-Tagatose

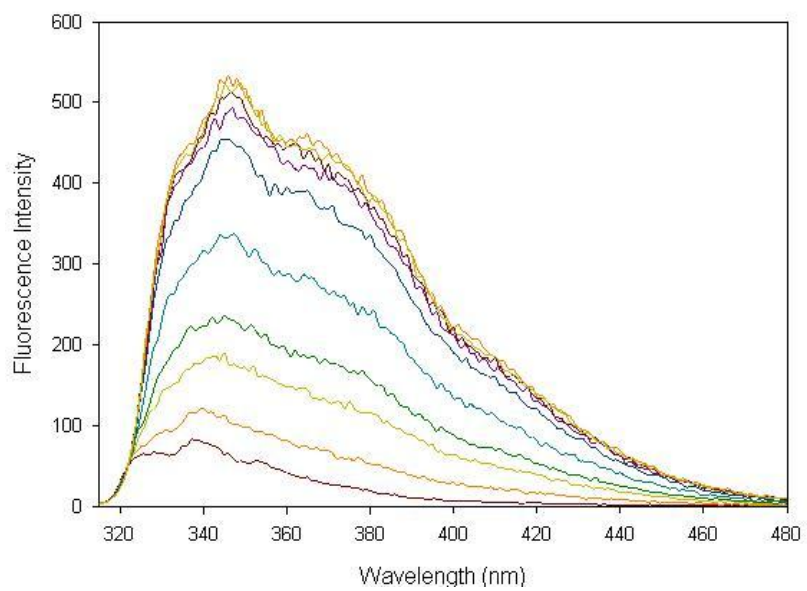

Figure A5. Fluorescent spectral changes of 7-IQBA $\left(1 \times 10^{-5} \mathrm{M}\right)$ upon addition of D-tagatose in phosphate buffer $(0.1 \mathrm{M})$ at $\mathrm{pH} 7.4: \lambda_{\mathrm{ex}}=272 \mathrm{~nm}, \lambda_{\mathrm{em}}=346 \mathrm{~nm}$. 
Phenoxathiin-4-boronic acid + D-Fructose

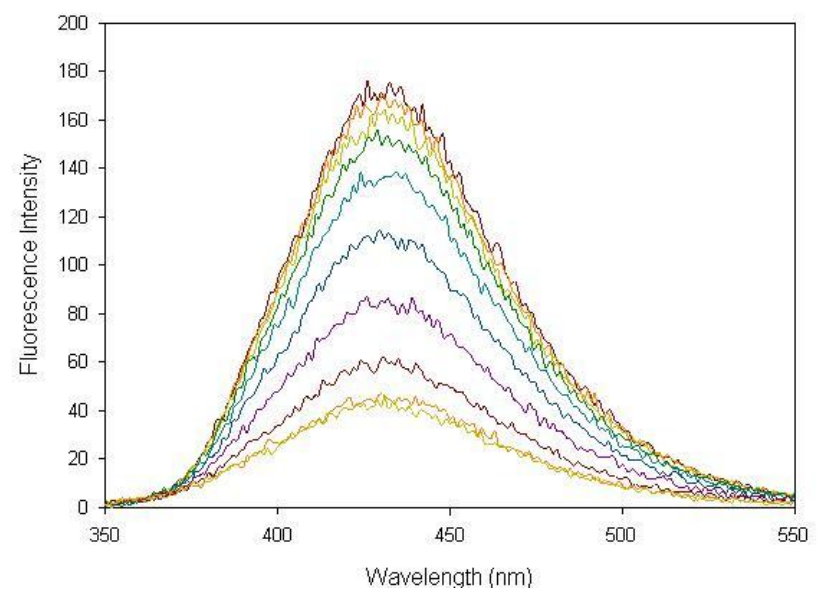

Phenoxathiin-4-boronic acid + D-Fructose ${ }^{[16]}$

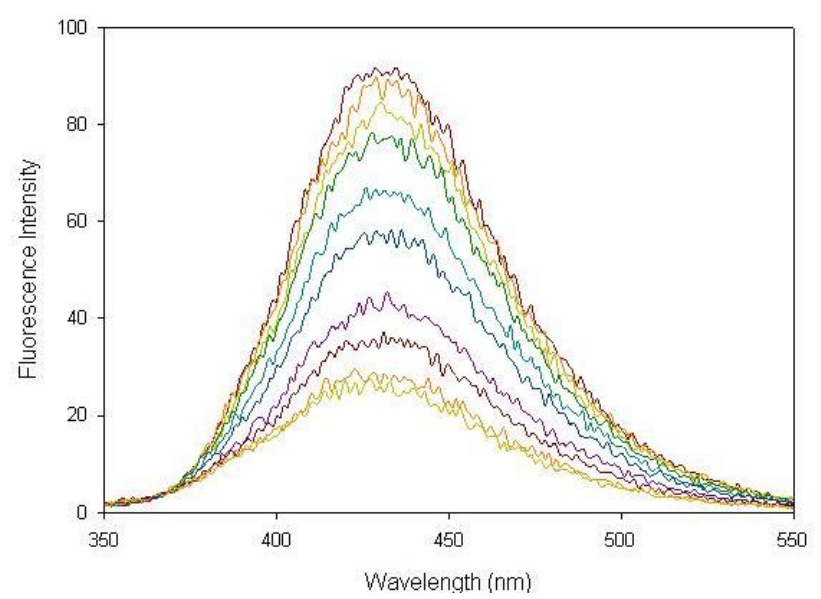

Phenoxathiin-4-boronic acid + D-Fructose

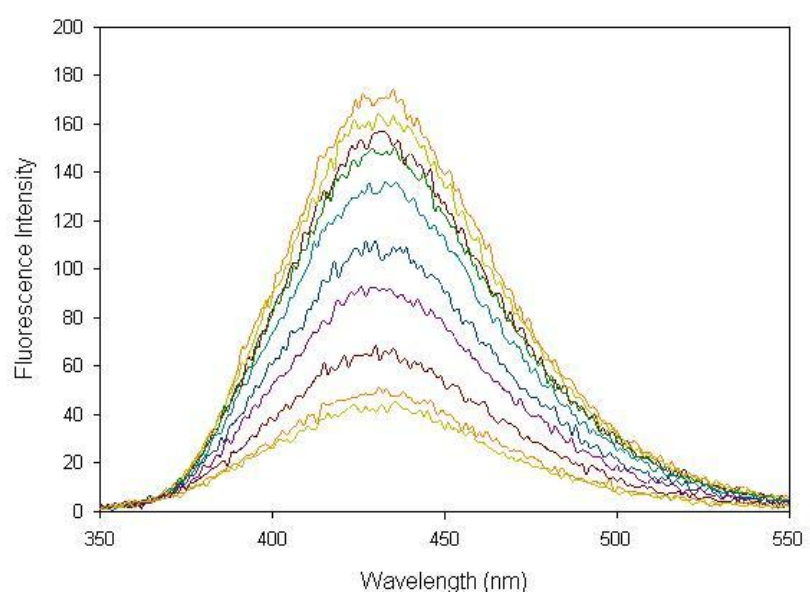

Figure A6. Fluorescent spectral changes of 4-POBA $\left(1 \times 10^{-5} \mathrm{M}\right)$ upon addition of D-fructose in phosphate buffer $(0.1 \mathrm{M})$ at $\mathrm{pH} 7.4: \lambda_{\mathrm{ex}}=299 \mathrm{~nm}, \lambda_{\mathrm{em}}=430 \mathrm{~nm}$. 
Phenoxathiin-4-boronic acid + D-Glucose

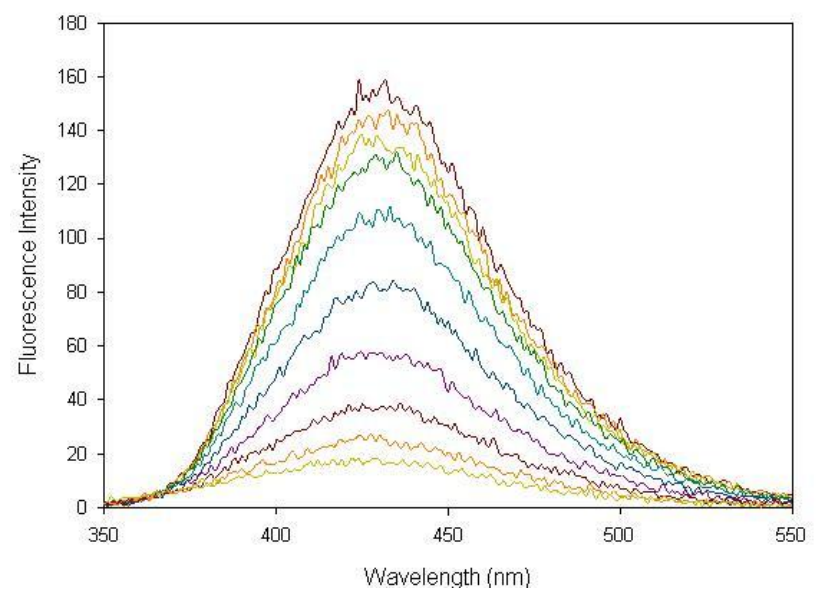

Phenoxathiin-4-boronic acid + D-Glucose ${ }^{[16]}$

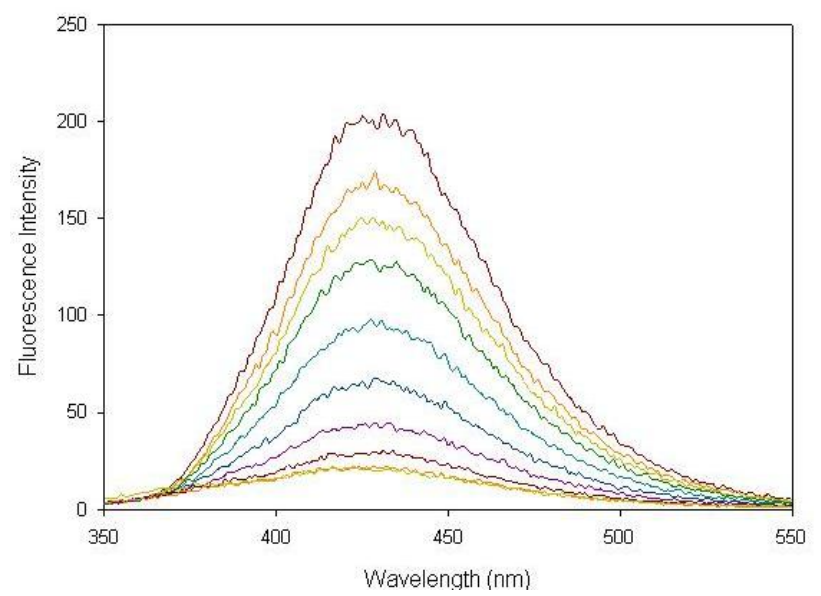

Phenoxathiin-4-boronic acid + D-Glucose ${ }^{[16]}$

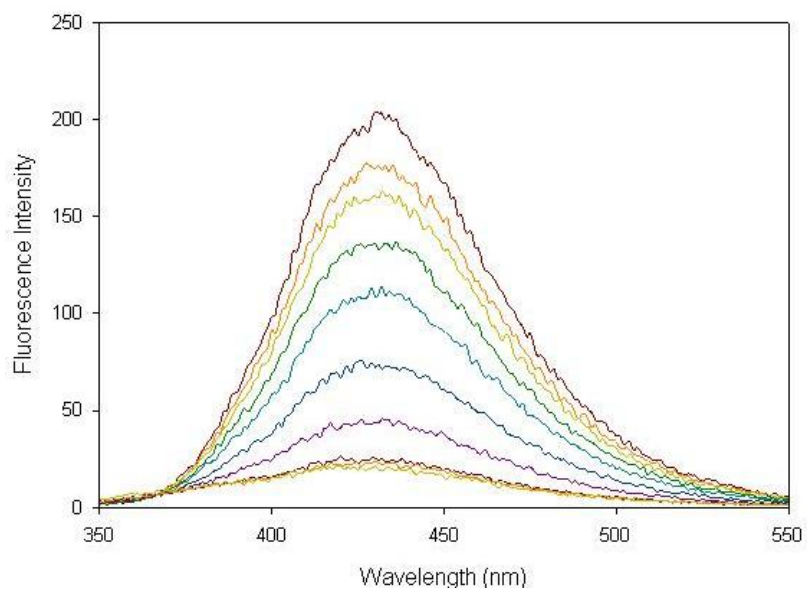

Figure A7. Fluorescent spectral changes of 4-POBA $\left(1 \times 10^{-5} \mathrm{M}\right)$ upon addition of D-glucose in phosphate buffer $(0.1 \mathrm{M})$ at $\mathrm{pH} 7.4: \lambda_{\mathrm{ex}}=299 \mathrm{~nm}, \lambda_{\mathrm{em}}=430 \mathrm{~nm}$. 
Phenoxathiin-4-boronic acid + D-Mannose

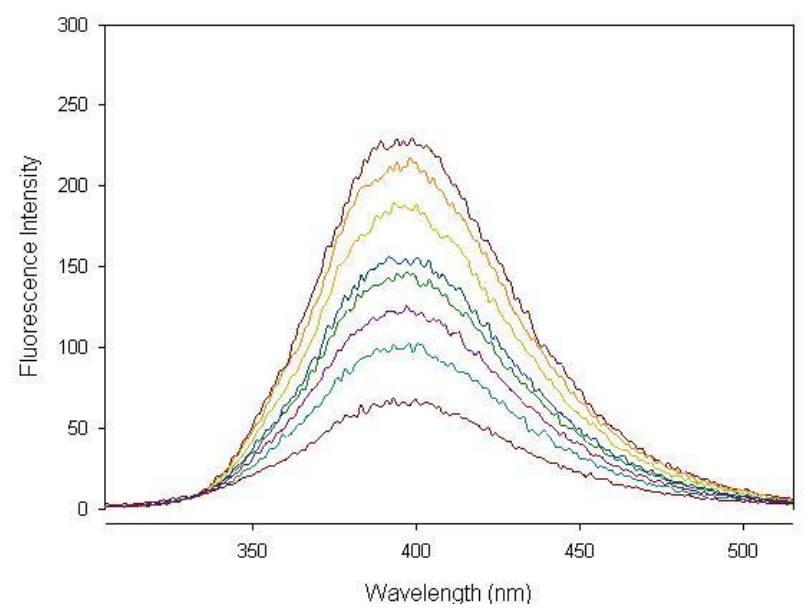

Phenoxathiin-4-boronic acid + D-Mannose

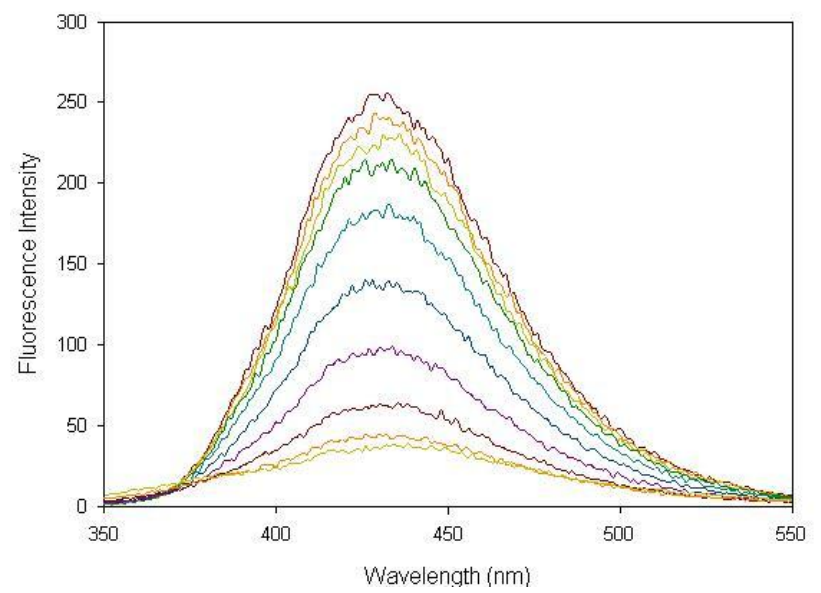

Phenoxathiin-4-boronic acid + D-Mannose

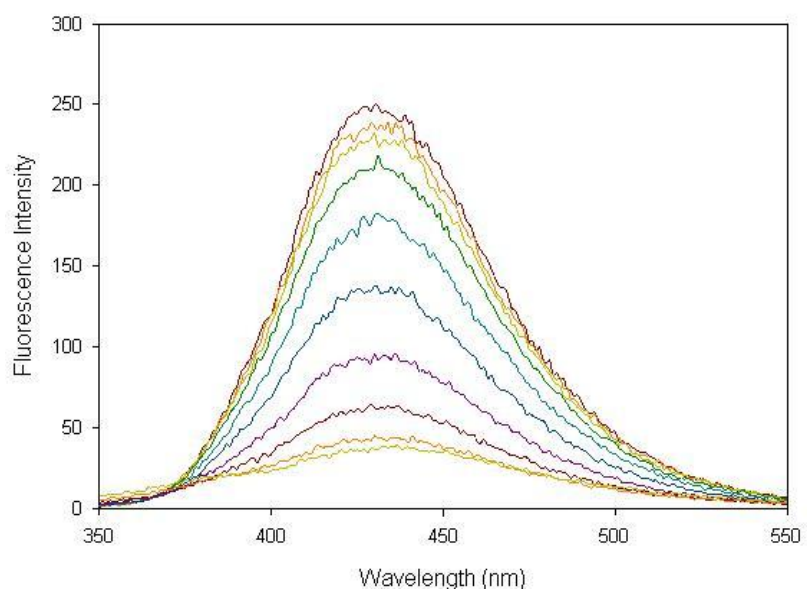

Figure A8. Fluorescent spectral changes of 4-POBA $\left(1 \times 10^{-5} \mathrm{M}\right)$ upon addition of D-mannose in phosphate buffer $(0.1 \mathrm{M})$ at $\mathrm{pH}$ 7.4: $\lambda_{\mathrm{ex}}=299 \mathrm{~nm}, \lambda_{\mathrm{em}}=430 \mathrm{~nm}$. 
Phenoxathiin-4-boronic acid + D-Sorbitol ${ }^{[16]}$

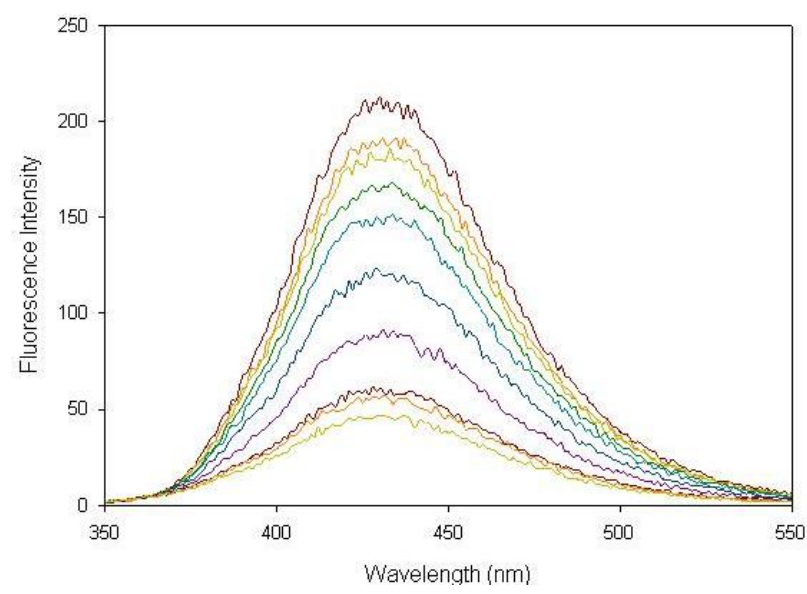

Phenoxathiin-4-boronic acid + D-Sorbitol ${ }^{[16]}$

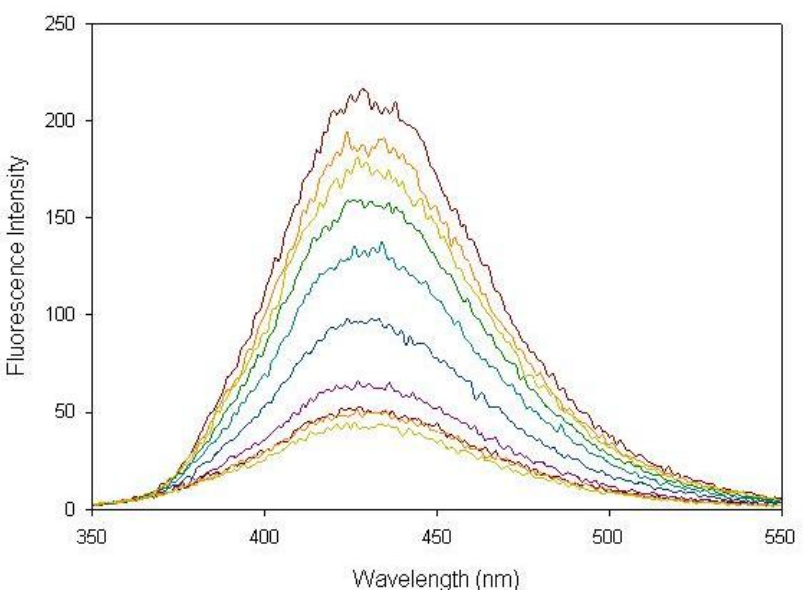

Phenoxathiin-4-boronic acid + D-Sorbitol ${ }^{[16]}$

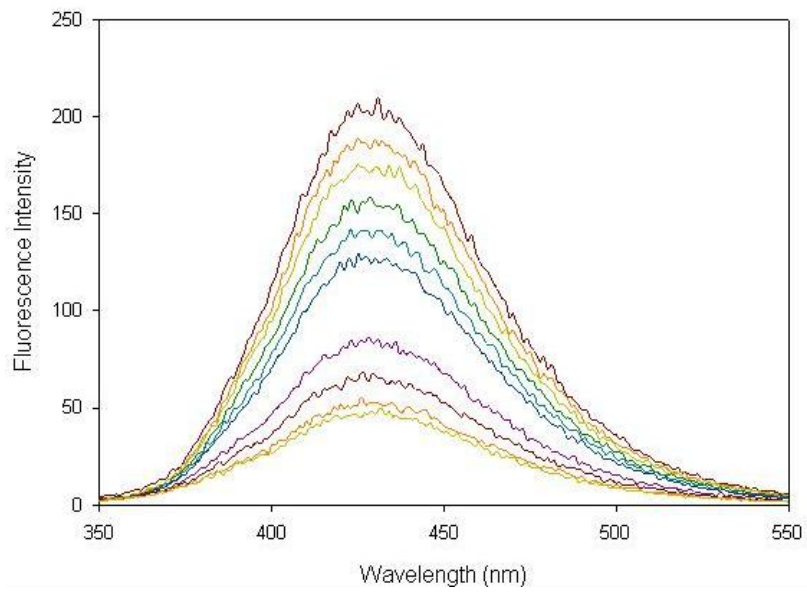

Figure A9. Fluorescent spectral changes of 4-POBA $\left(1 \times 10^{-5} \mathrm{M}\right)$ upon addition of D-sorbitol in phosphate buffer $(0.1 \mathrm{M})$ at $\mathrm{pH}$ 7.4: $\lambda_{\mathrm{ex}}=299 \mathrm{~nm}, \lambda_{\mathrm{em}}=430 \mathrm{~nm}$. 
Phenoxathiin-4-boronic acid + D-Tagatose

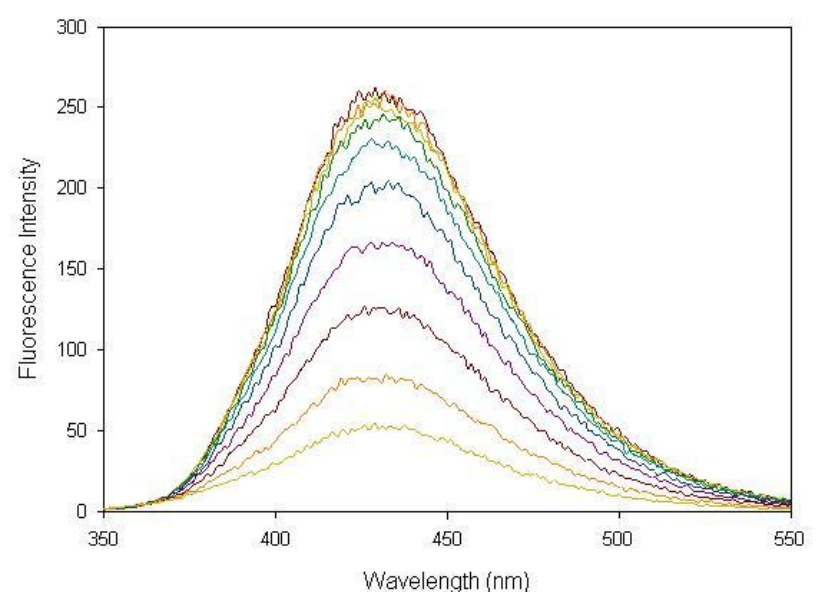

Phenoxathiin-4-boronic acid + D-Tagatose

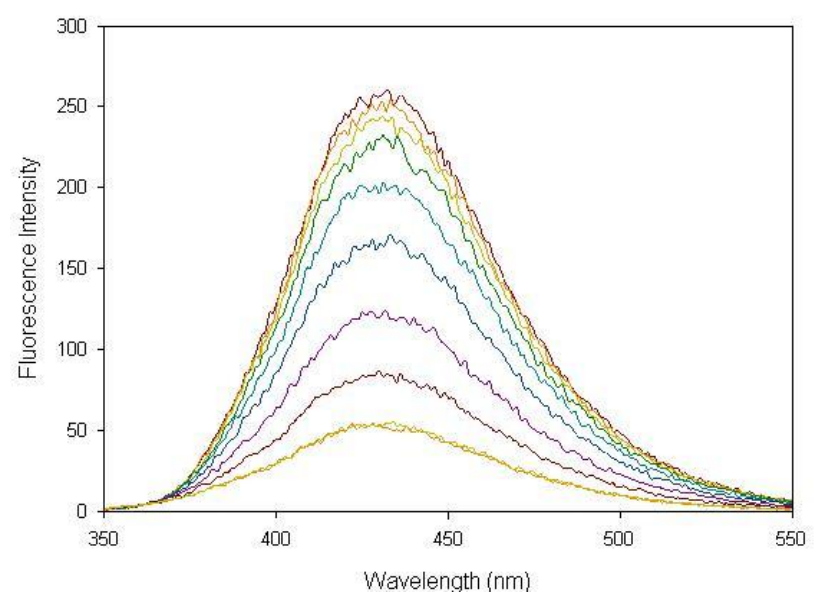

Phenoxathiin-4-boronic acid + D-Tagatose

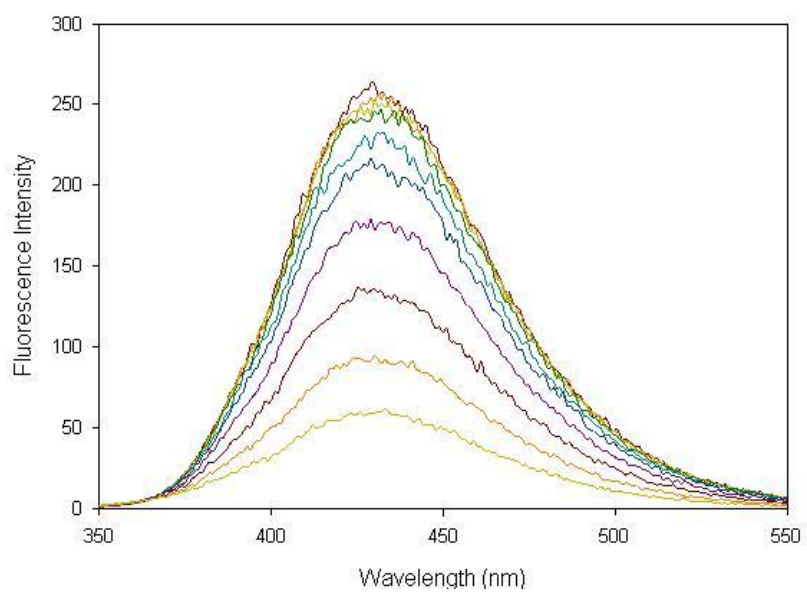

Figure A10. Fluorescent spectral changes of 4-POBA $\left(1 \times 10^{-5} \mathrm{M}\right)$ upon addition of D-tagatose in phosphate buffer $(0.1 \mathrm{M})$ at $\mathrm{pH} 7.4: \lambda_{\mathrm{ex}}=299 \mathrm{~nm}, \lambda_{\mathrm{em}}=430 \mathrm{~nm}$. 


\section{APPENDIX B - Fluorescence Quantum Yield $\left(\Phi_{\mathrm{F}}\right)$}

Isoquinoline-7-boronic acid

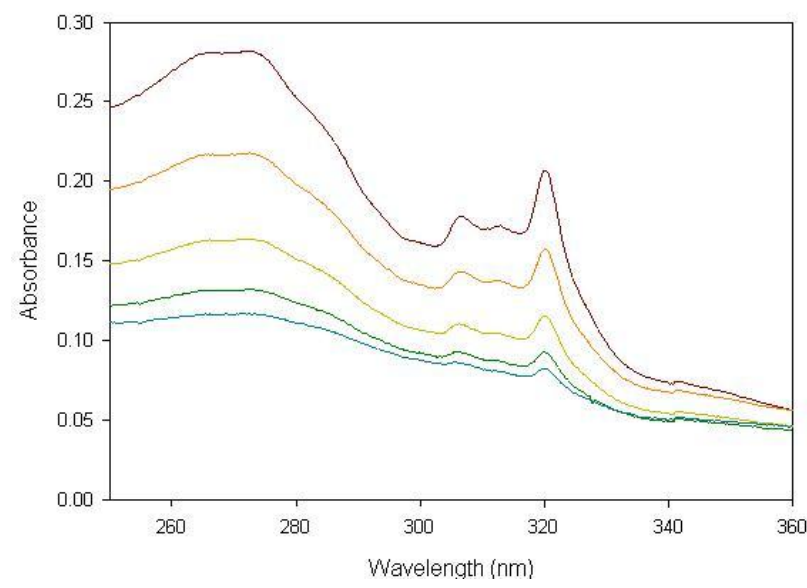

Isoquinoline-7-boronic acid

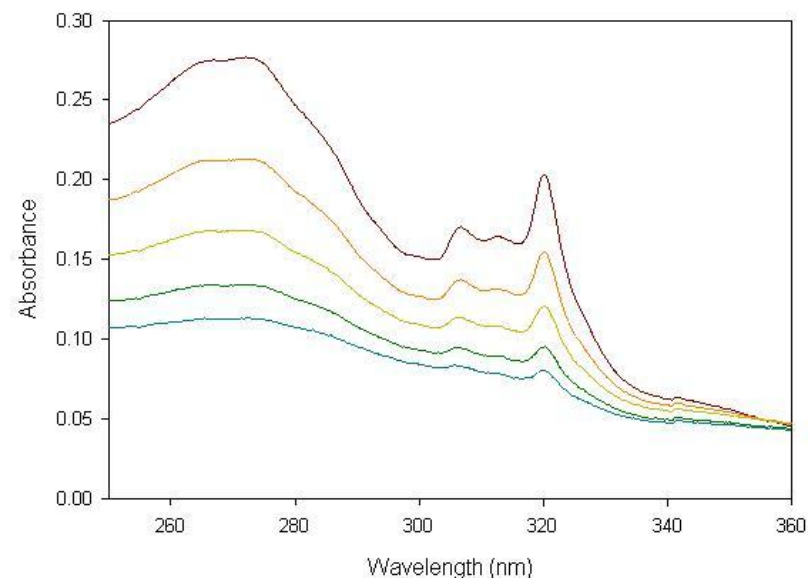

Isoquinoline-7-boronic acid

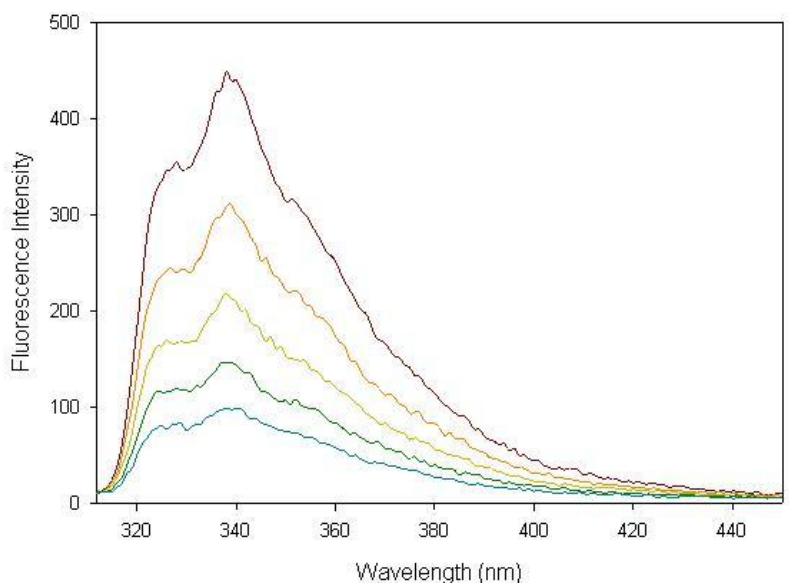

Isoquinoline-7-boronic acid

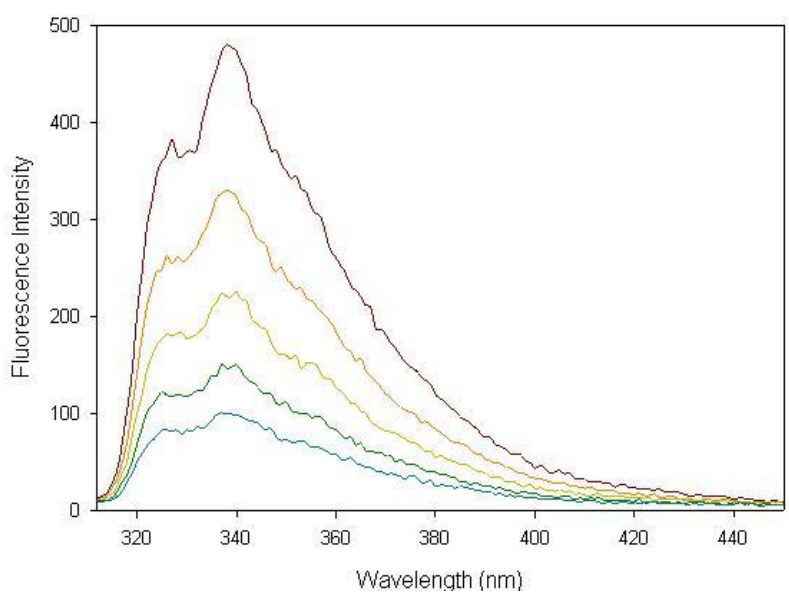

Figure B1. Absorbance and fluorescence spectra of decreasing 7-IQBA concentrations (initial: $1 \times$ $10^{-5} \mathrm{M}$ with $1 / 3$ serial dilutions) in the absence of sugar in phosphate buffer $(0.1 \mathrm{M})$ at $\mathrm{pH}$ 7.4: $\lambda_{\mathrm{ex}}=$ $272 \mathrm{~nm}$, area range $\lambda_{\mathrm{em}}=275-500 \mathrm{~nm}$. 
Isoquinoline-7-boronic acid + D-Fructose ${ }^{[16]}$

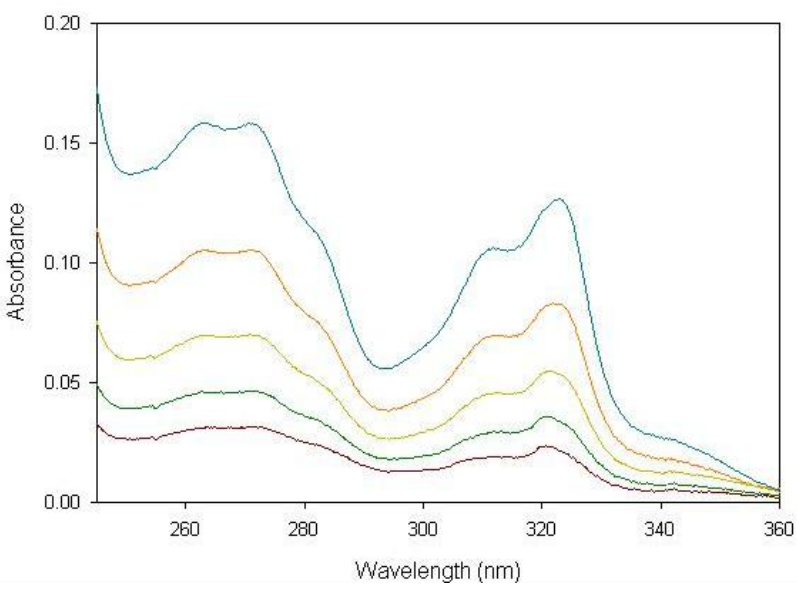

Isoquinoline-7-boronic acid + D-Fructose ${ }^{[16]}$

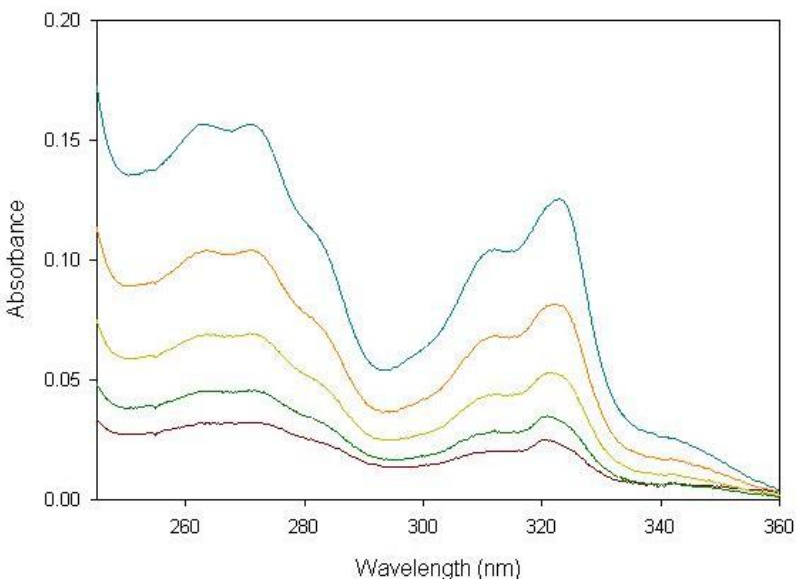

Isoquinoline-7-boronic acid + D-Fructose ${ }^{[16]}$

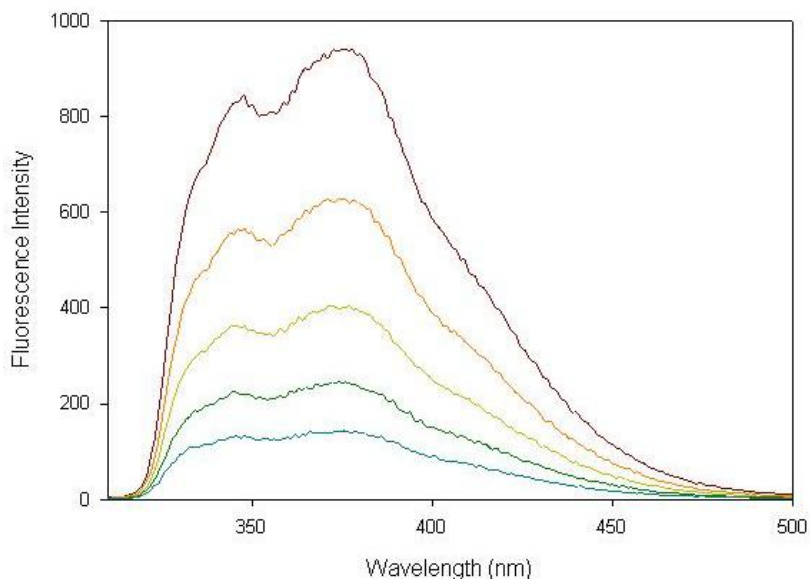

Isoquinoline-7-boronic acid + D-Fructose ${ }^{[16]}$

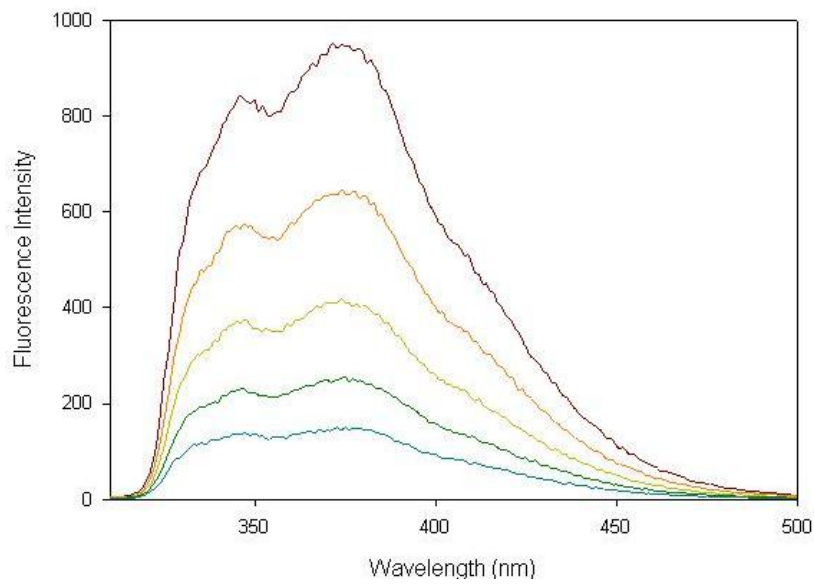

Figure B2. Absorbance and fluorescence spectra of decreasing 7-IQBA concentrations (initial: $1 \times$ $10^{-5} \mathrm{M}$ with $1 / 3$ serial dilutions) in the presence of $\mathrm{D}$-fructose in phosphate buffer $(0.1 \mathrm{M})$ at $\mathrm{pH} 7.4$ : $\lambda_{\mathrm{ex}}=272 \mathrm{~nm}$, area range $\lambda_{\mathrm{em}}=310-500 \mathrm{~nm}$. 
Isoquinoline-7-boronic acid + D-Mannose

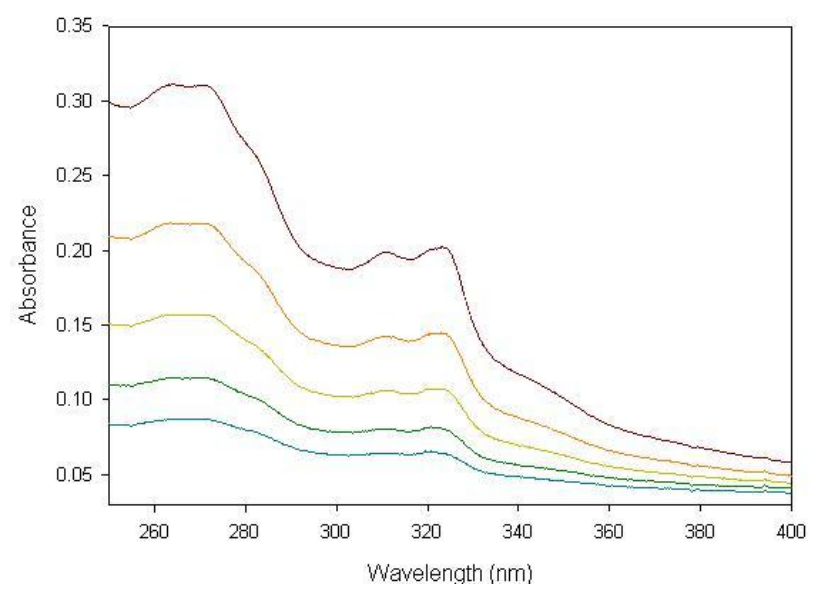

Isoquinoline-7-boronic acid + D-Mannose

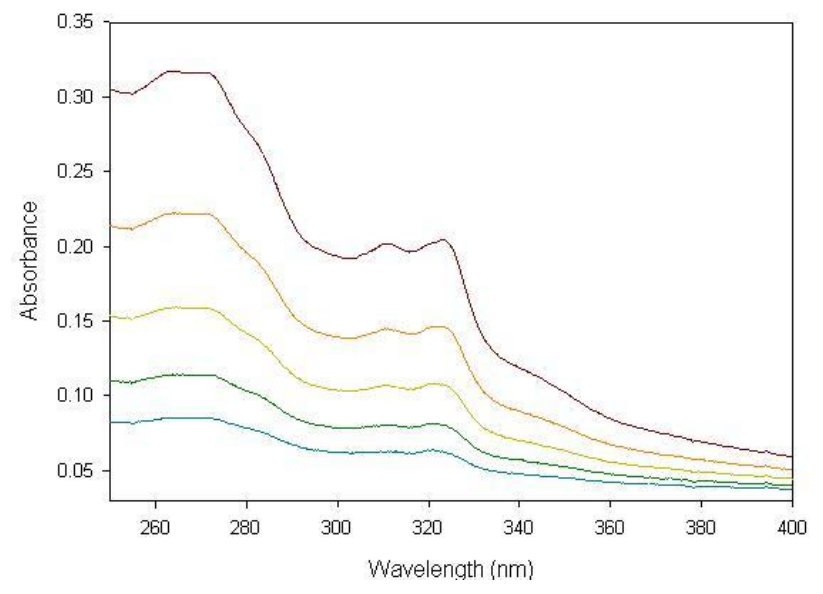

Isoquinoline-7-boronic acid + D-Mannose

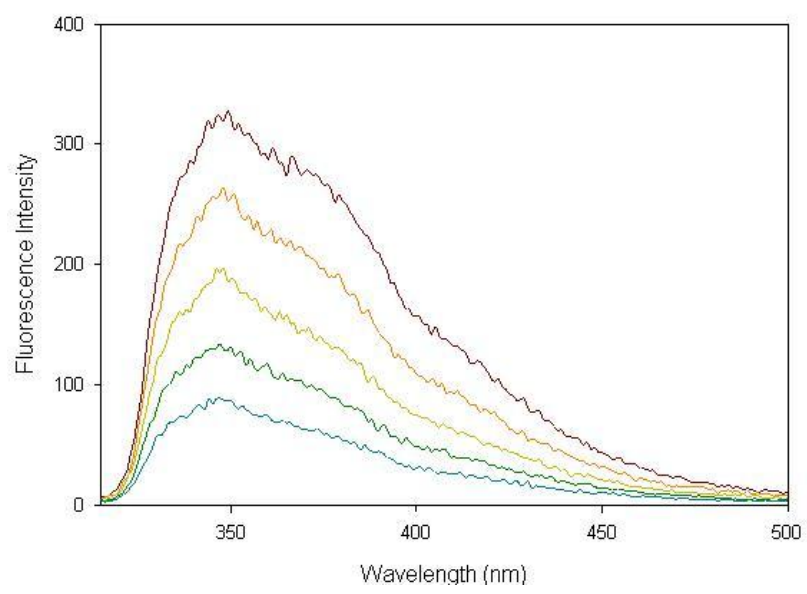

Isoquinoline-7-boronic acid + D-Mannose

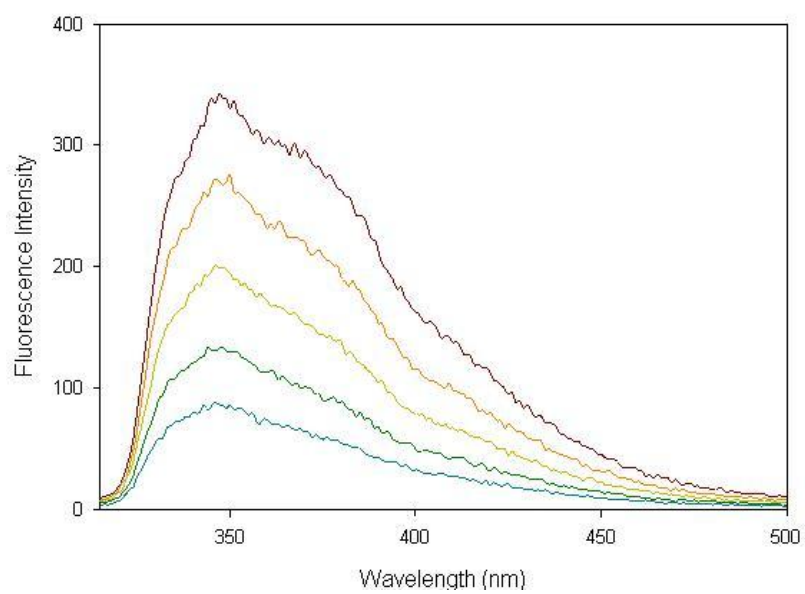

Figure B3. Absorbance and fluorescence spectra of decreasing 7-IQBA concentrations (initial: $1 \times$ $10^{-5} \mathrm{M}$ with $1 / 3$ serial dilutions) in the presence of $\mathrm{D}$-mannose in phosphate buffer $(0.1 \mathrm{M})$ at $\mathrm{pH}$ 7.4: $\lambda_{\mathrm{ex}}=272 \mathrm{~nm}$, area range $\lambda_{\mathrm{em}}=315-500 \mathrm{~nm}$. 
Isoquinoline-7-boronic acid + D-Sorbitol ${ }^{[16]}$

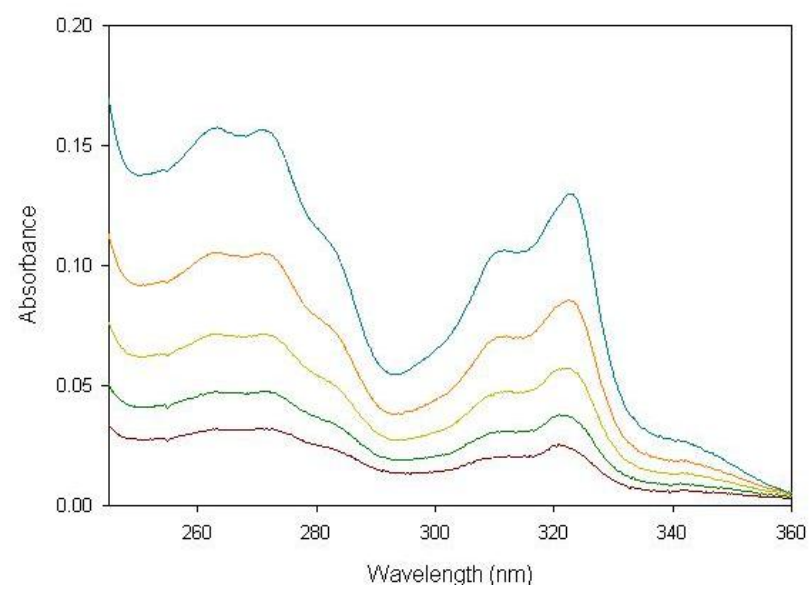

Isoquinoline-7-boronic acid + D-Sorbitol ${ }^{[16]}$

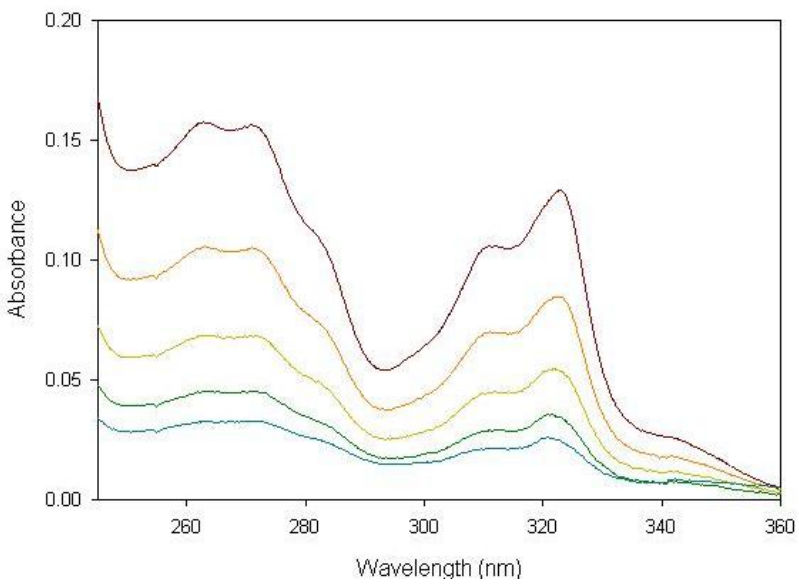

Isoquinoline-7-boronic acid + D-Sorbitol ${ }^{[16]}$

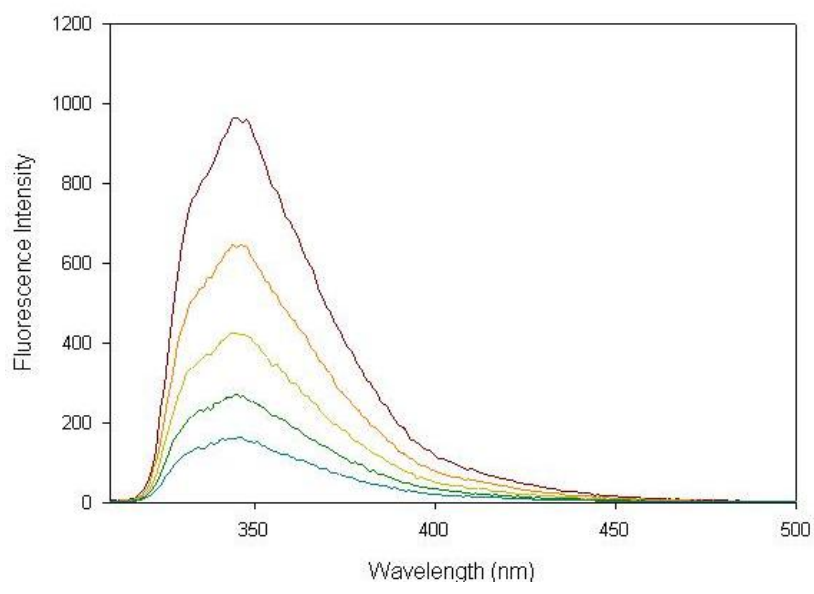

Isoquinoline-7-boronic acid + D-Sorbitol ${ }^{[16]}$

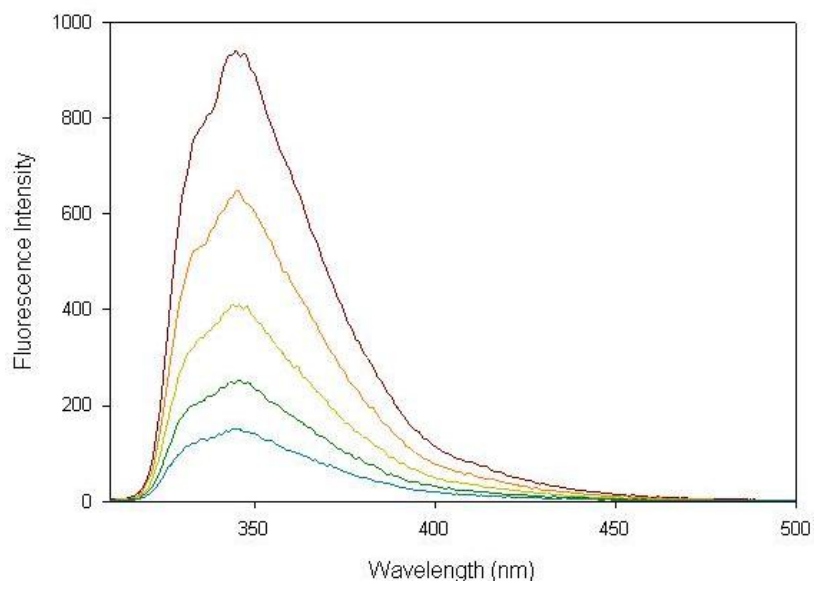

Figure B4. Absorbance and fluorescence spectra of decreasing 7-IQBA concentrations (initial: $1 \times$ $10^{-5} \mathrm{M}$ with $1 / 3$ serial dilutions) in the presence of $\mathrm{D}$-sorbitol in phosphate buffer $(0.1 \mathrm{M})$ at $\mathrm{pH} 7.4$ : $\lambda_{\mathrm{ex}}=272 \mathrm{~nm}$, area range $\lambda_{\mathrm{em}}=310-500 \mathrm{~nm}$. 
Isoquinoline-7-boronic acid + D-Tagatose

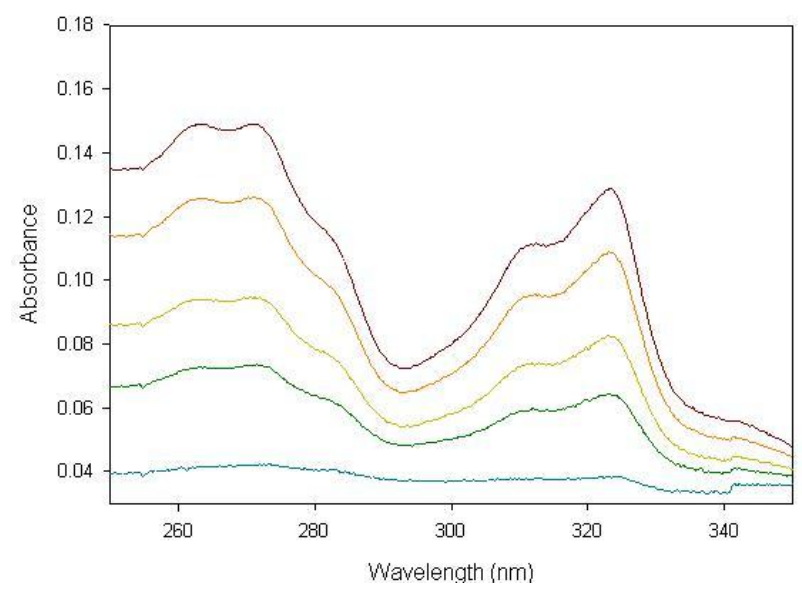

Isoquinoline-7-boronic acid + D-Tagatose

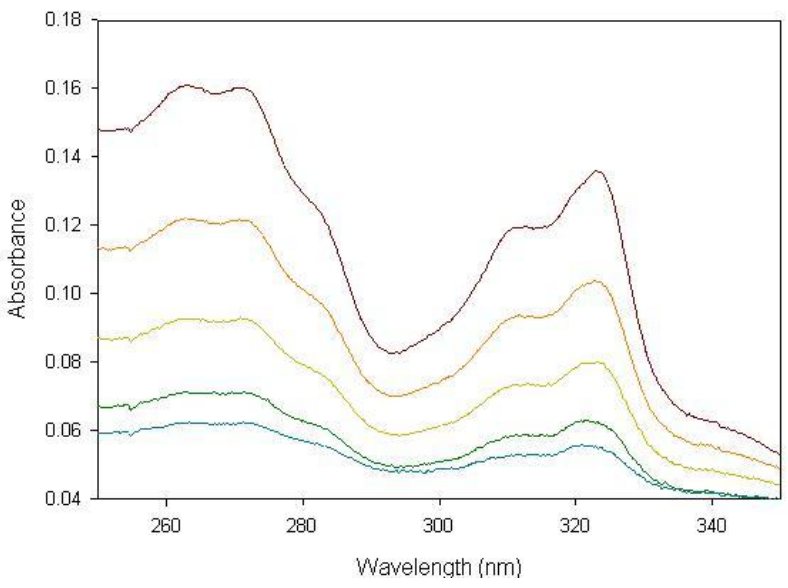

Isoquinoline-7-boronic acid + D-Tagatose

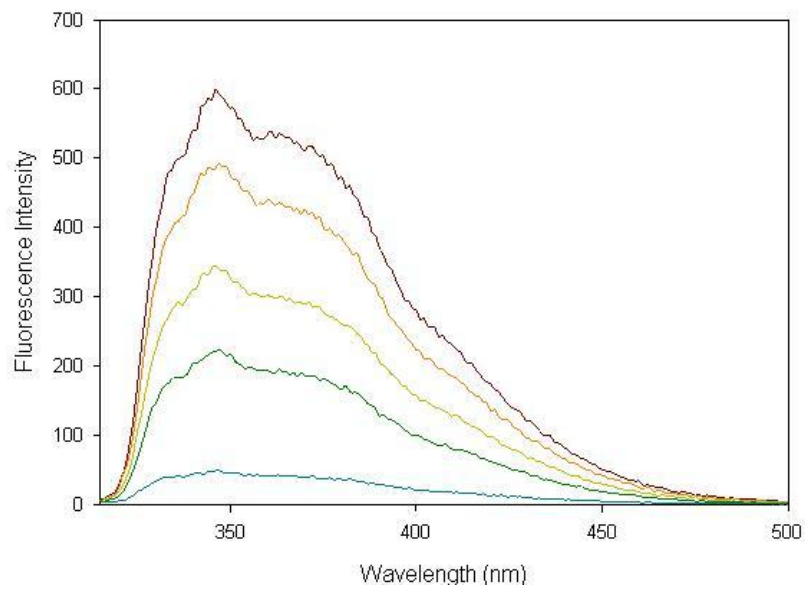

Isoquioline-7-boronic acid + D-Tagatose

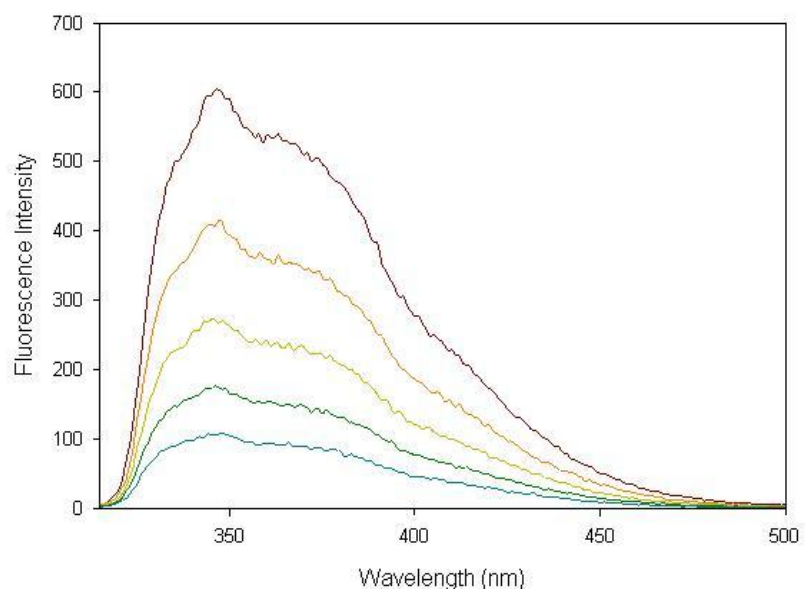

Figure B5. Absorbance and fluorescence spectra of decreasing 7-IQBA concentrations (initial: $1 \times$ $10^{-5} \mathrm{M}$ with $1 / 3$ serial dilutions) in the presence of $\mathrm{D}$-tagatose in phosphate buffer $(0.1 \mathrm{M})$ at $\mathrm{pH} 7.4$ : $\lambda_{\mathrm{ex}}=272 \mathrm{~nm}$, area range $\lambda_{\mathrm{em}}=315-500 \mathrm{~nm}$. 
Phenoxathiin-4-boronic acid

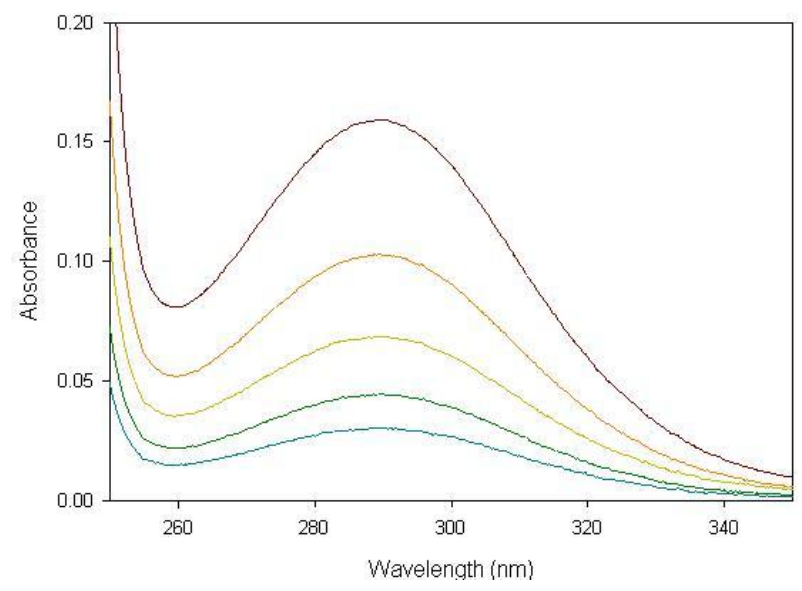

Phenoxathiin-4-boronic acid

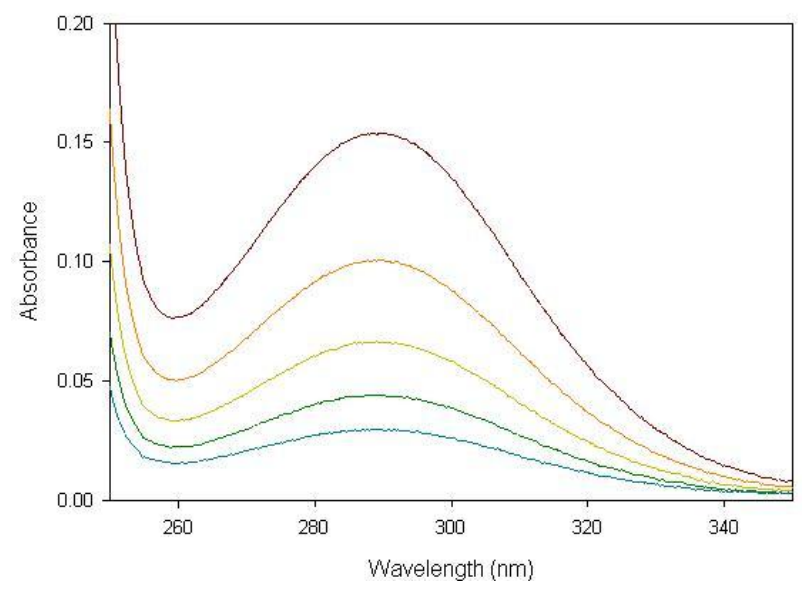

Phenoxathiin-4-boronic acid

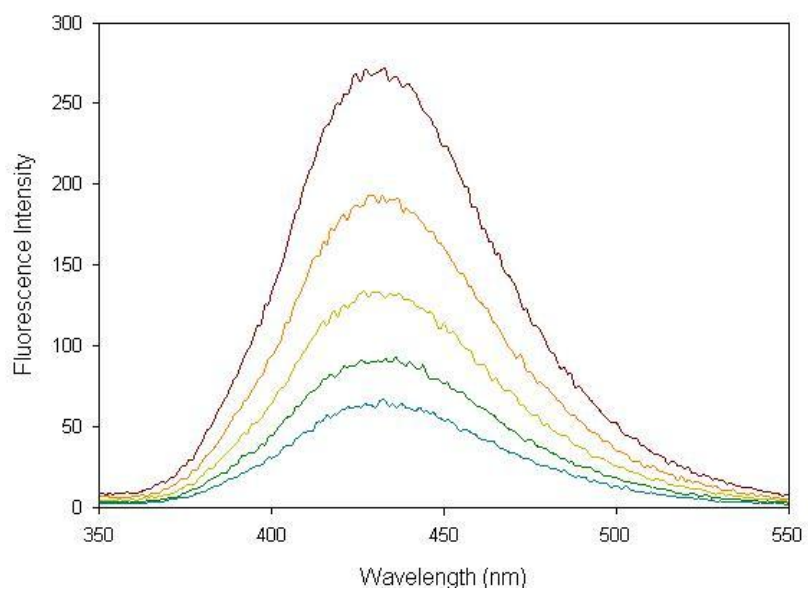

Phenoxathiin-4-boronic acid

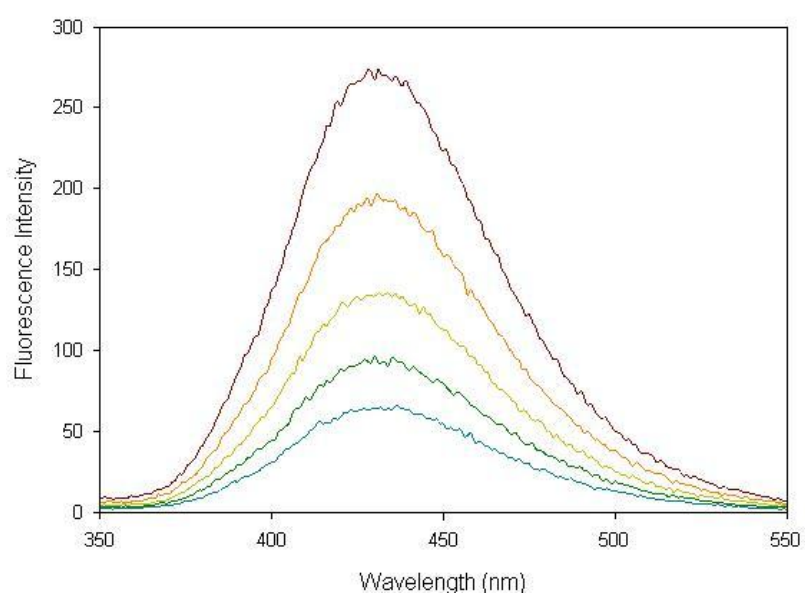

Figure B6. Absorbance and fluorescence spectra of decreasing 4-POBA concentrations (initial: $1 \times$ $10^{-5} \mathrm{M}$ with $1 / 3$ serial dilutions) in the absence of sugar in phosphate buffer $(0.1 \mathrm{M})$ at $\mathrm{pH} 7.4: \lambda_{\mathrm{ex}}=$ $299 \mathrm{~nm}$, area range $\lambda_{\mathrm{em}}=350-550 \mathrm{~nm}$. 
Phenoxathiin-4-boronic acid + D-Fructose ${ }^{[16]}$

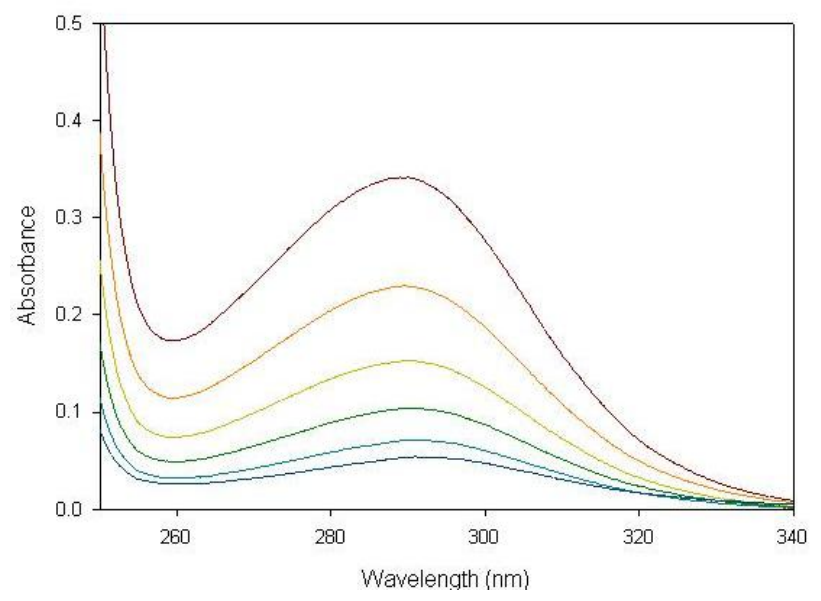

Phenoxathiin-4-boronic acid + D-Fructose ${ }^{[16]}$

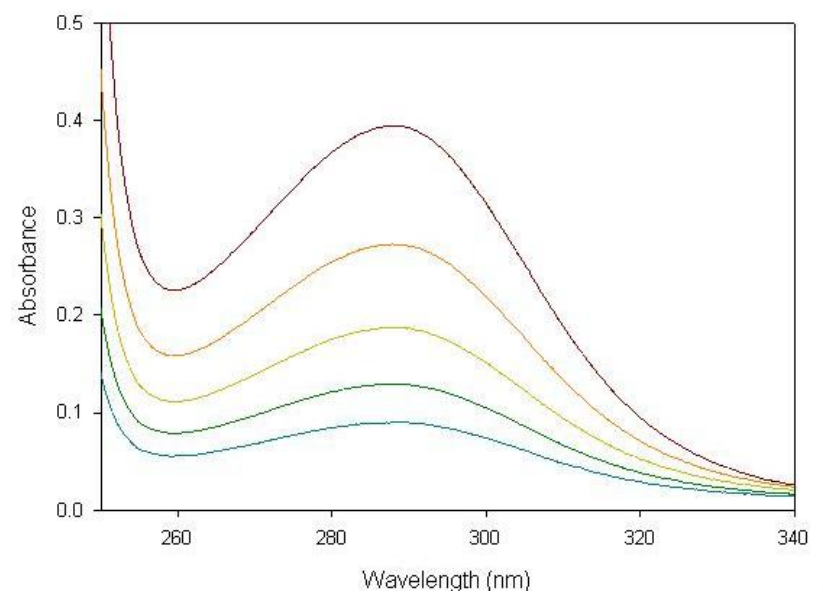

Phenoxathiin-4-boronic acid + D-Fructose ${ }^{[16]}$

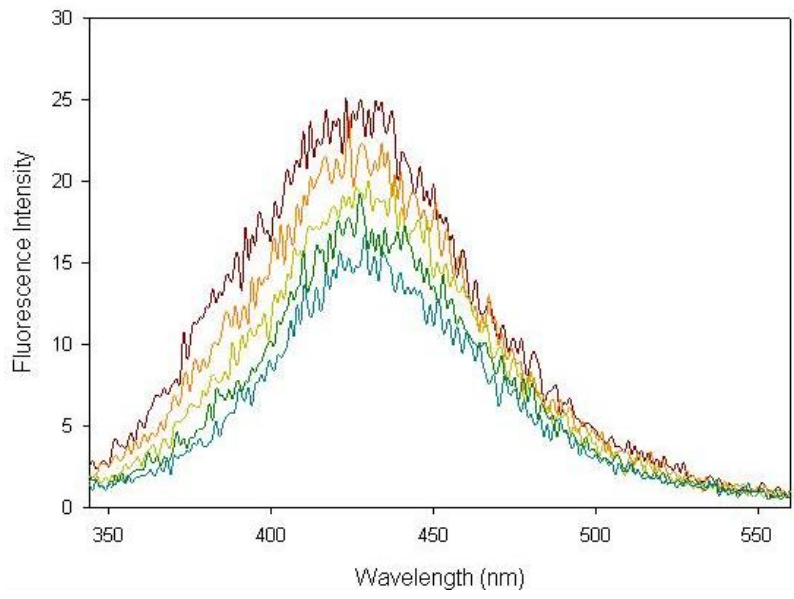

Phenoxathiin-4-boronic acid + D-Fructose ${ }^{[16]}$

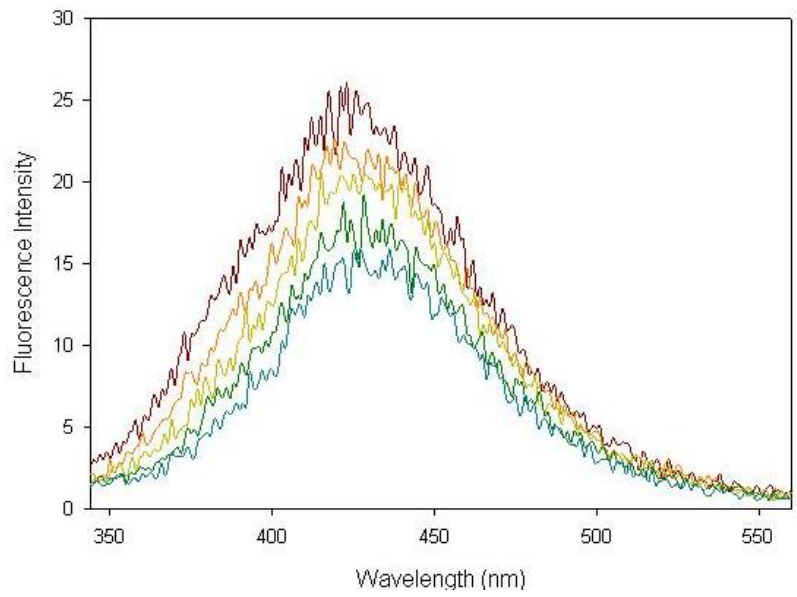

Figure B7. Absorbance and fluorescence spectra of decreasing 4-POBA concentrations (initial: $1 \times$ $10^{-5} \mathrm{M}$ with $1 / 3$ serial dilutions) in the presence of $\mathrm{D}$-fructose in phosphate buffer $(0.1 \mathrm{M})$ at $\mathrm{pH}$ 7.4: $\lambda_{\mathrm{ex}}=299 \mathrm{~nm}$, area range $\lambda_{\mathrm{em}}=344-560 \mathrm{~nm}$. 
Phenoxathiin-4-boronic acid + D-Glucose ${ }^{[16]}$

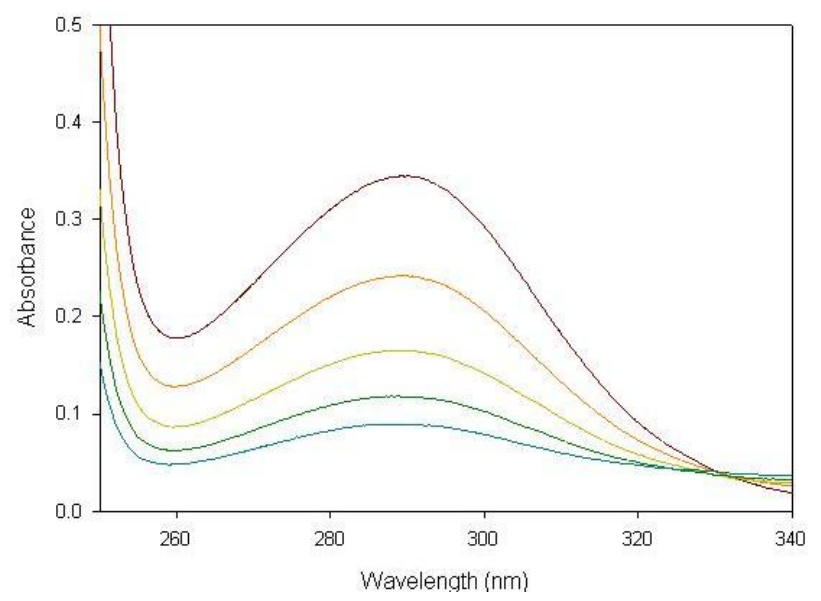

Phenoxathiin-4-boronic acid + D-Glucose ${ }^{[16]}$

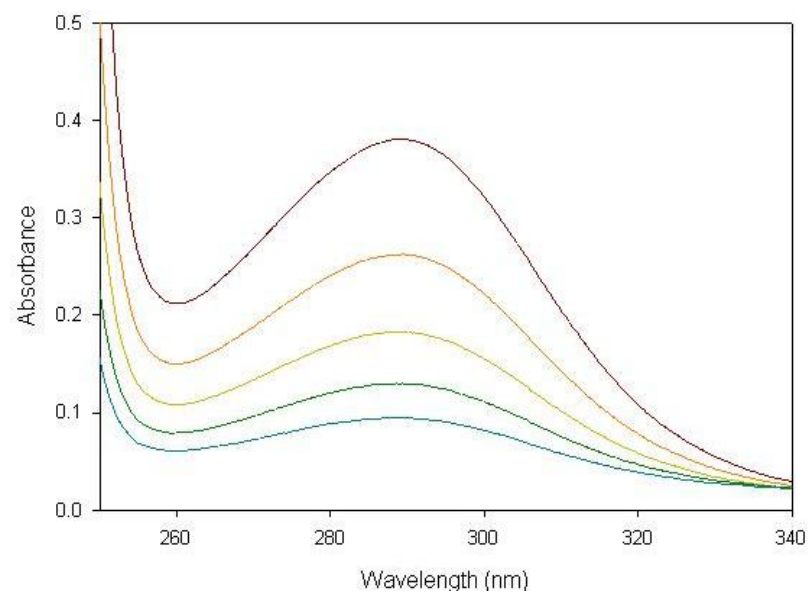

Phenoxathiin-4-boronic acid + D-Glucose ${ }^{[16]}$

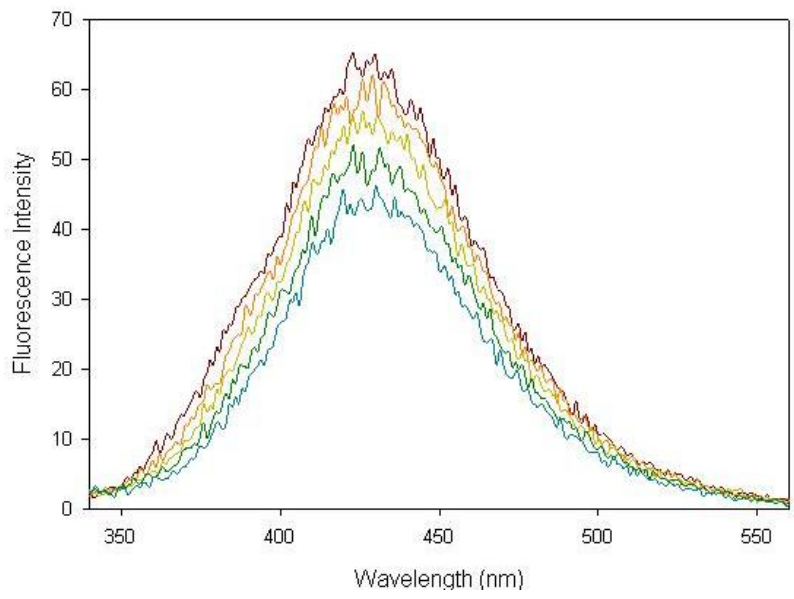

Phenoxathiin-4-boronic acid + D-Glucose ${ }^{[16]}$

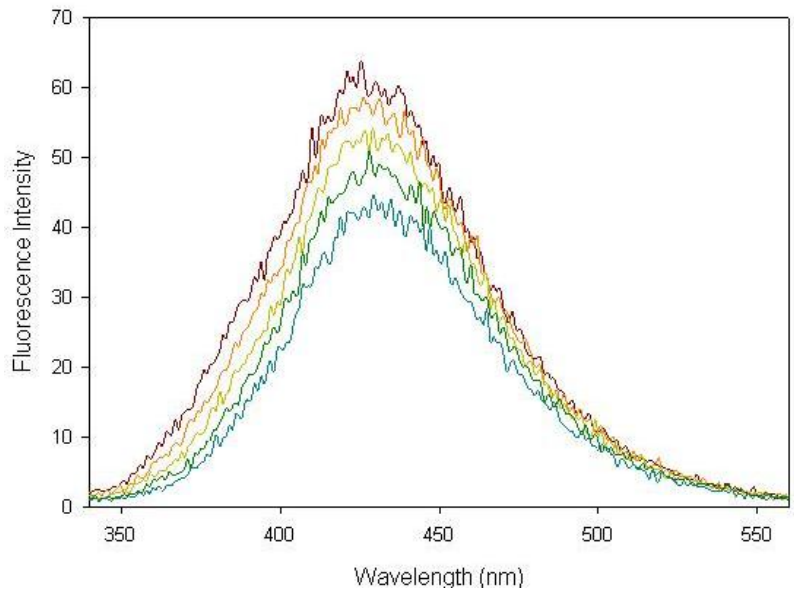

Figure B8. Absorbance and fluorescence spectra of decreasing 4-POBA concentrations (initial: $1 \times$ $10^{-5} \mathrm{M}$ with $1 / 3$ serial dilutions) in the presence of $\mathrm{D}$-glucose in phosphate buffer $(0.1 \mathrm{M})$ at $\mathrm{pH} 7.4$ : $\lambda_{\mathrm{ex}}=299 \mathrm{~nm}$, area range $\lambda_{\mathrm{em}}=340-560 \mathrm{~nm}$. 
Phenoxathiin-4-boronic acid + D-Mannose ${ }^{[16]}$

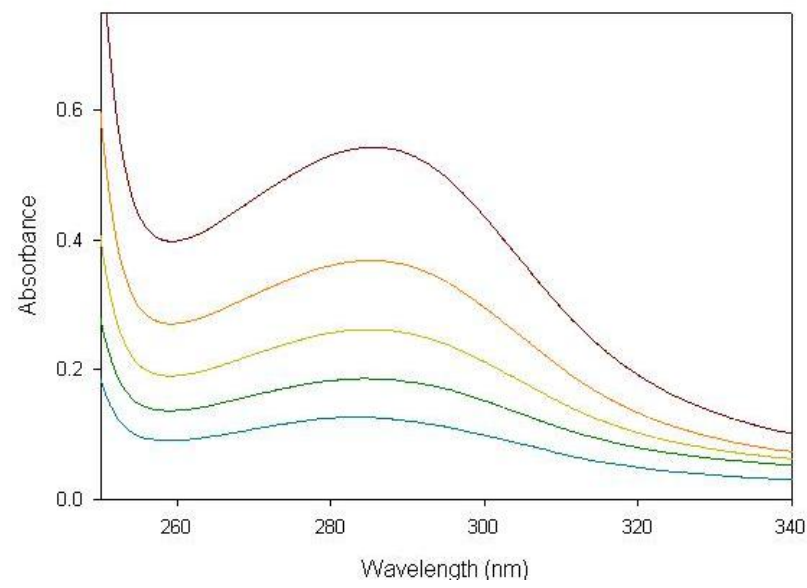

Phenoxathiin-4-boronic acid + D-Mannose ${ }^{[16]}$

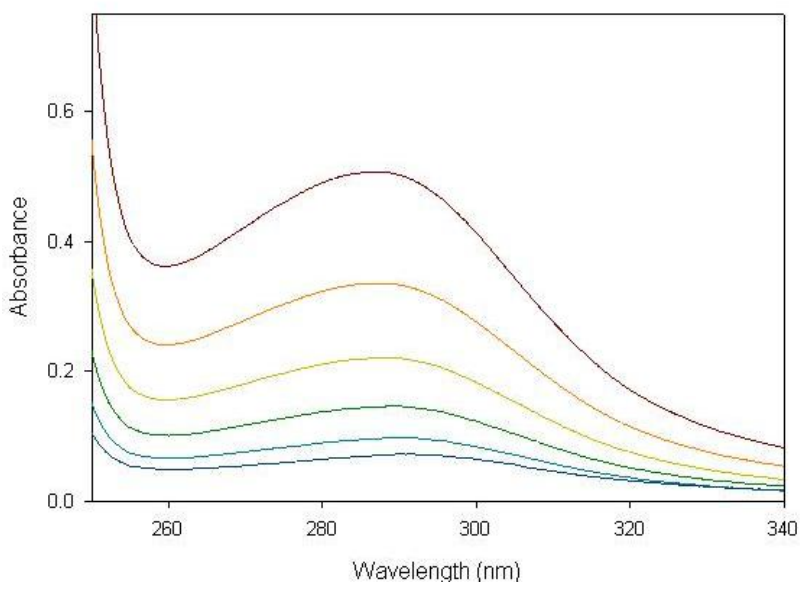

Phenoxathiin-4-boronic acid + D-Mannose ${ }^{[16]}$

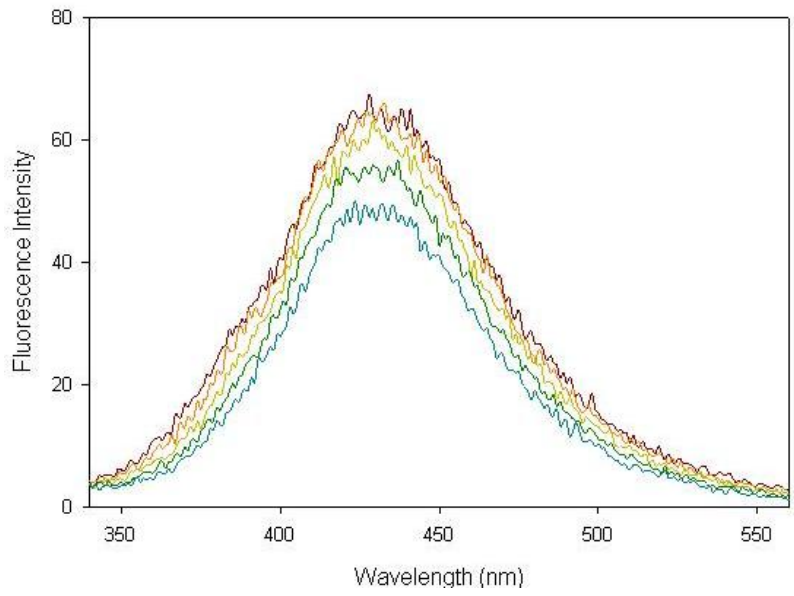

Phenoxathiin-4-boronic acid + D-Mannose ${ }^{[16]}$

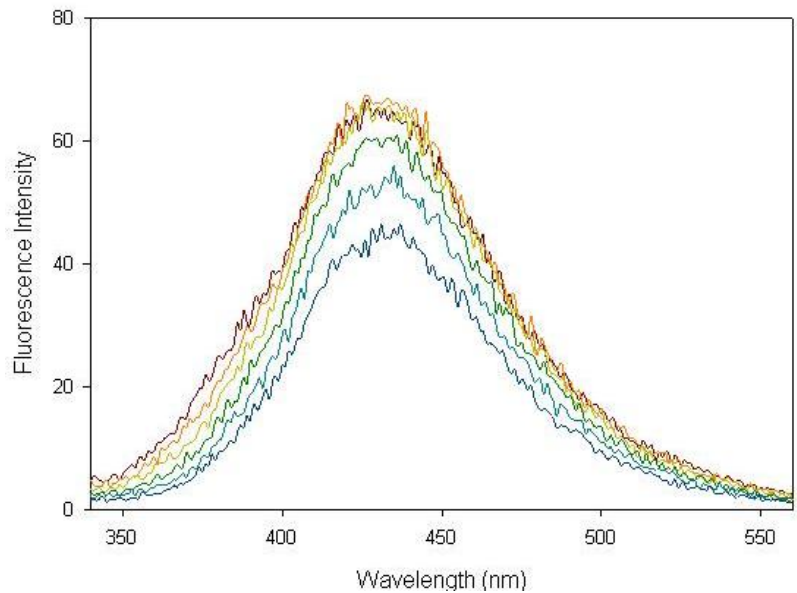

Figure B9. Absorbance and fluorescence spectra of decreasing 4-POBA concentrations (initial: $1 \times$ $10^{-5} \mathrm{M}$ with $1 / 3$ serial dilutions) in the presence of $\mathrm{D}$-mannose in phosphate buffer $(0.1 \mathrm{M})$ at $\mathrm{pH}$ 7.4: $\lambda_{\mathrm{ex}}=299 \mathrm{~nm}$, area range $\lambda_{\mathrm{em}}=340-560 \mathrm{~nm}$. 
Phenoxathiin-4-boronic acid + D-Sorbitol ${ }^{[16]}$

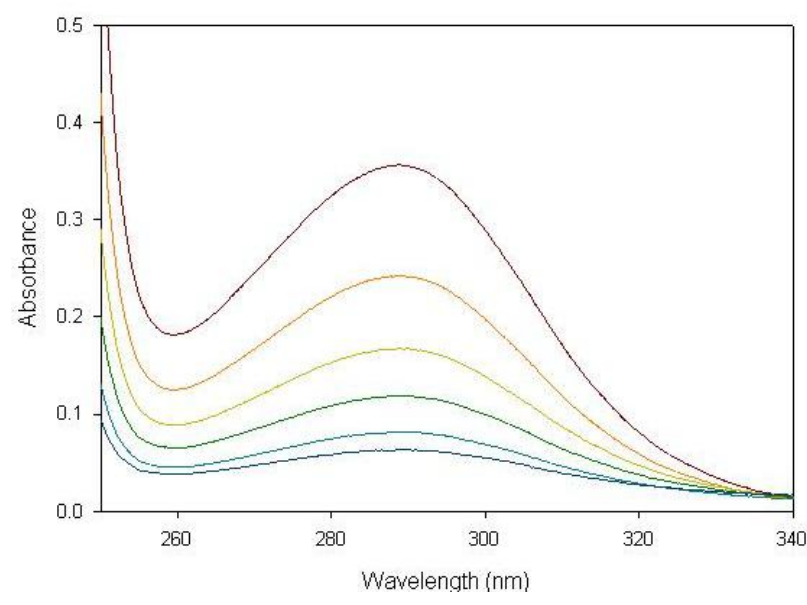

Phenoxathiin-4-boronic acid + D-Sorbitol ${ }^{[16]}$

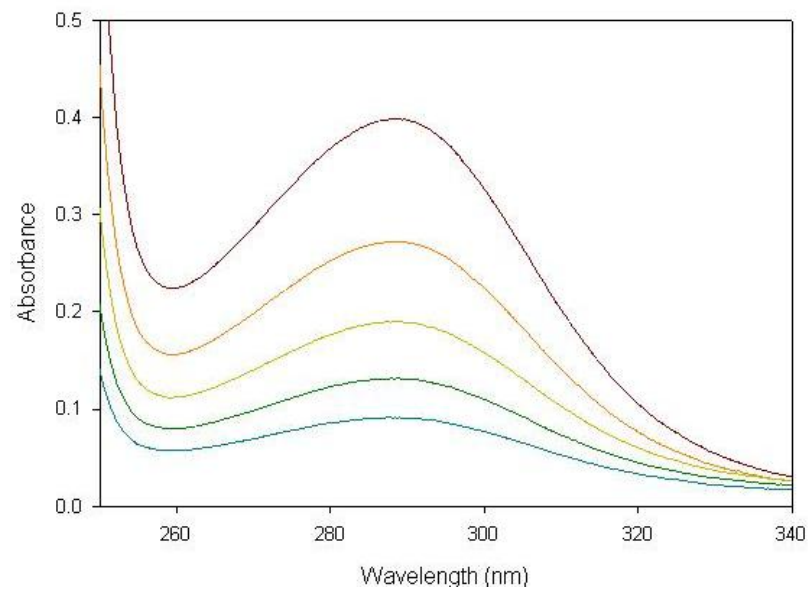

Phenoxathiin-4-boronic acid + D-Sorbitol ${ }^{[16]}$

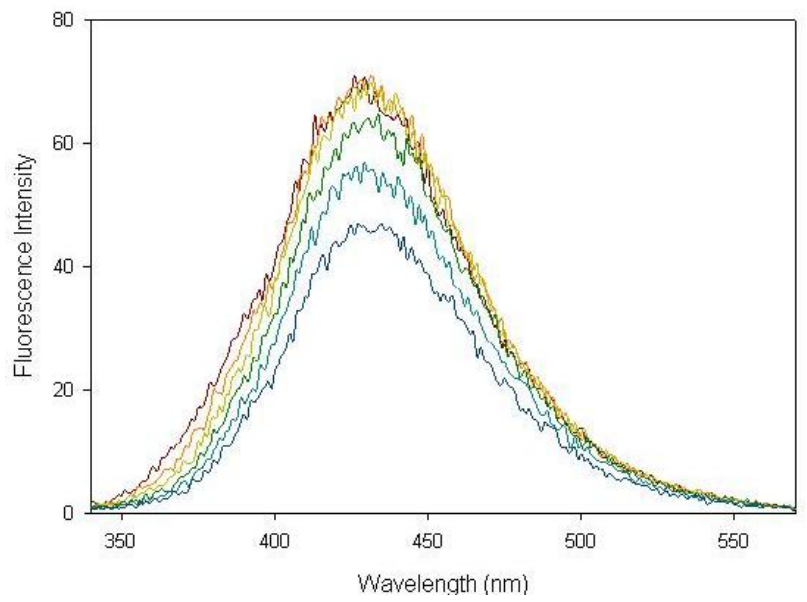

Phenoxathiin-4-boronic acid + D-Sorbitol ${ }^{[16]}$

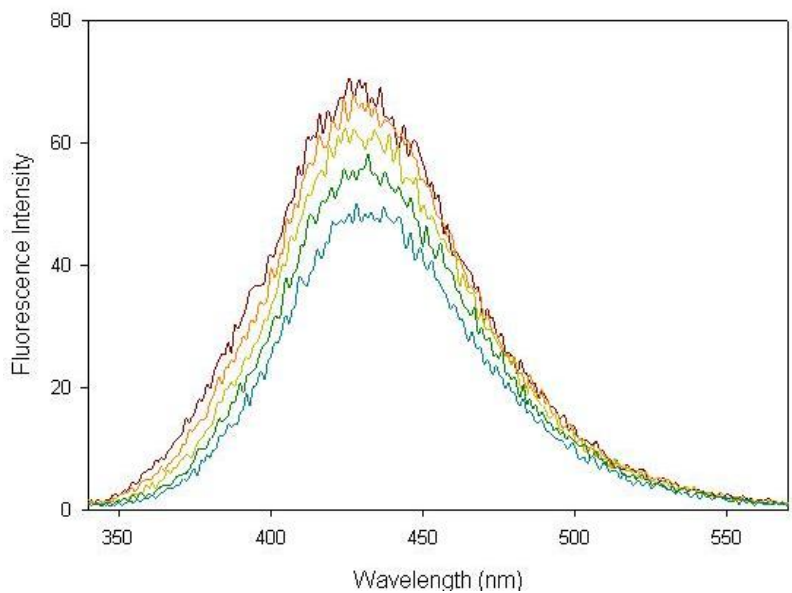

Figure B10. Absorbance and fluorescence spectra of decreasing 4-POBA concentrations (initial: 1 $\times 10^{-5} \mathrm{M}$ with $1 / 3$ serial dilutions) in the presence of $\mathrm{D}$-sorbitol in phosphate buffer $(0.1 \mathrm{M})$ at $\mathrm{pH}$ 7.4: $\lambda_{\mathrm{ex}}=299 \mathrm{~nm}$, area range $\lambda_{\mathrm{em}}=340-570 \mathrm{~nm}$. 
Phenoxathiin-4-boronic acid + D-Tagatose ${ }^{[16]}$

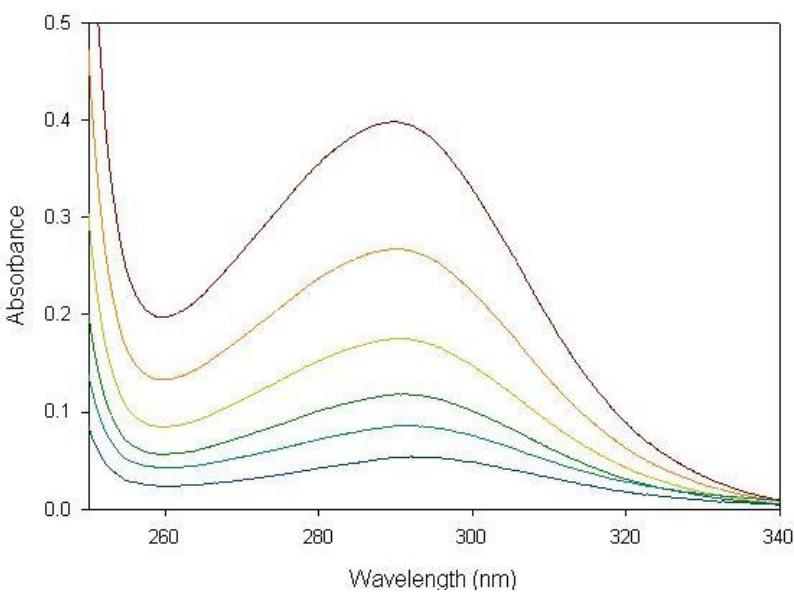

Phenoxathiin-4-boronic acid + D-Tagatose ${ }^{[16]}$

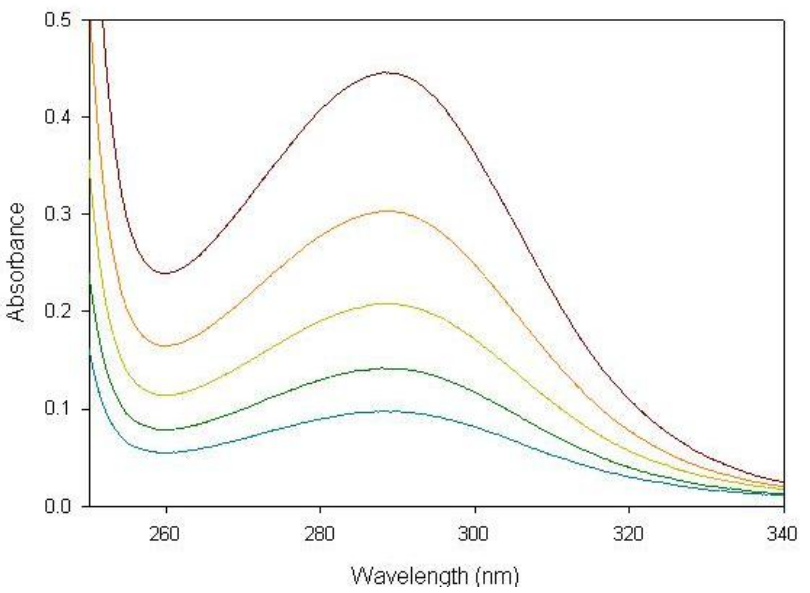

Phenoxathiin-4-boronic acid + D-Tagatose ${ }^{[16]}$

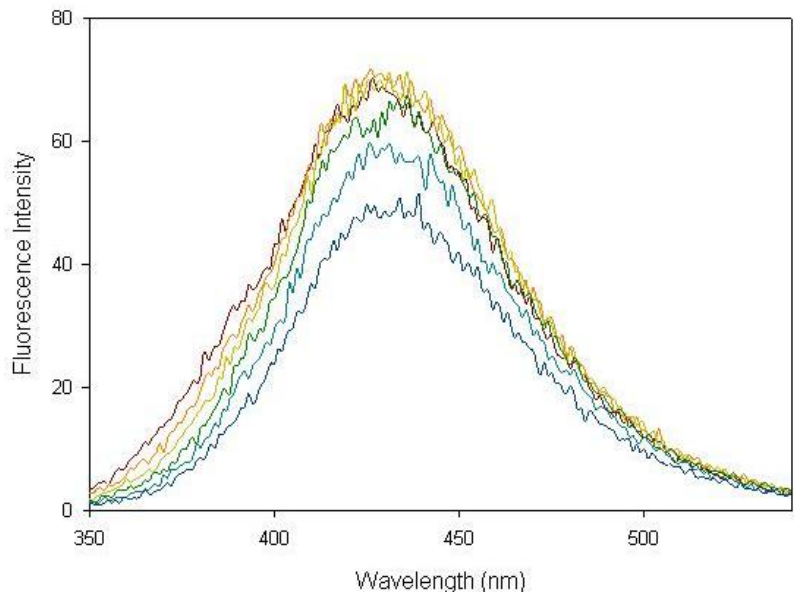

Phenoxathiin-4-boronic acid + D-Tagatose ${ }^{[16]}$

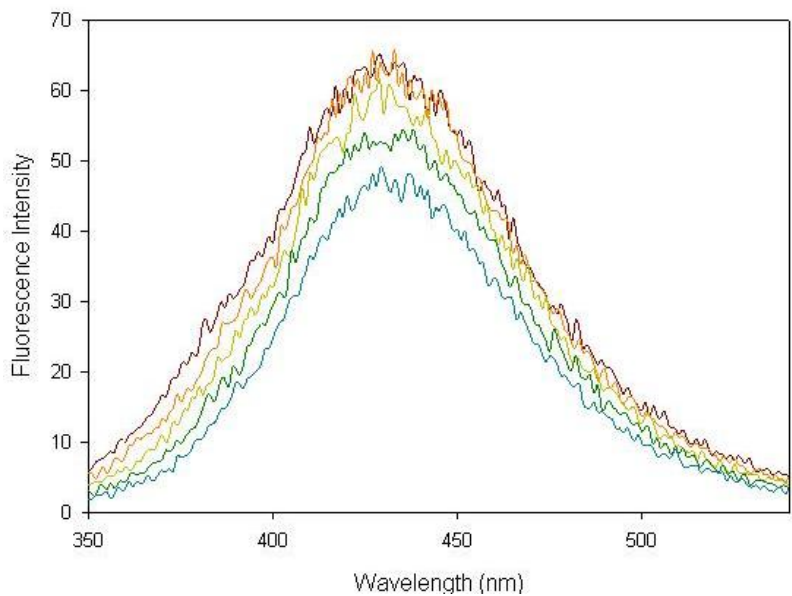

Figure B11. Absorbance and fluorescence spectra of decreasing 4-POBA concentrations (initial: 1 $\times 10^{-5} \mathrm{M}$ with $1 / 3$ serial dilutions) in the presence of $\mathrm{D}$-tagatose in phosphate buffer $(0.1 \mathrm{M})$ at pH 7.4: $\lambda_{\mathrm{ex}}=299 \mathrm{~nm}$, area range $\lambda_{\mathrm{em}}=350-540 \mathrm{~nm}$. 


\section{APPENDIX C - pH Profiles \& Identification of $\mathrm{p} K_{\mathrm{a}}$ Values}

Isoquinoline-7-boronic acid

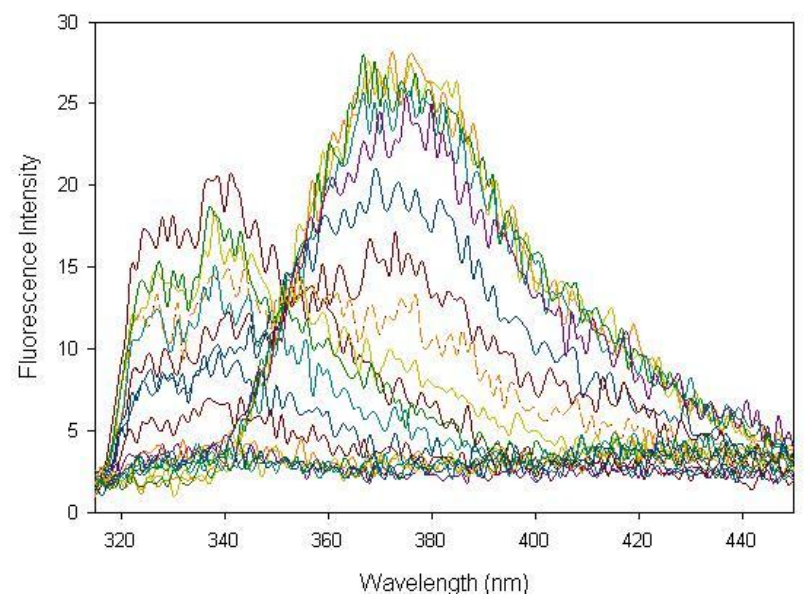

Isoquinoline-7-boronic acid

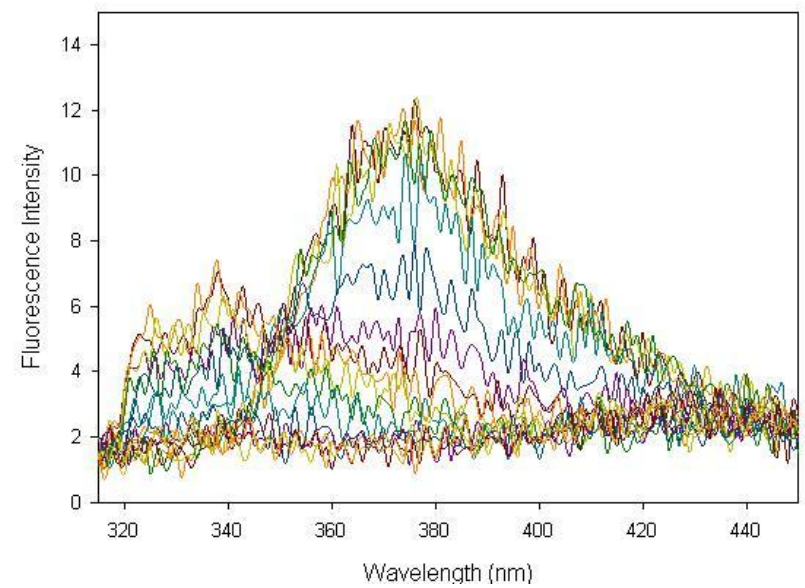

Isoquinoline-7-boronic acid

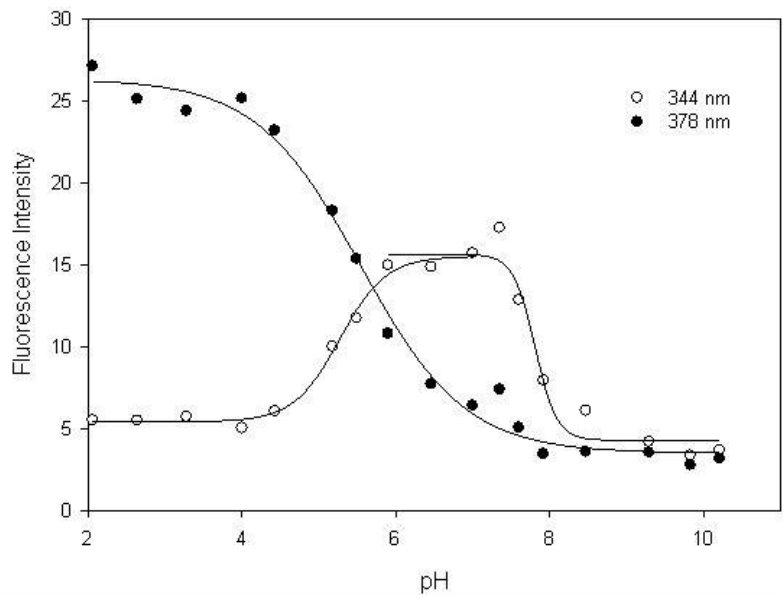

Isoquinoline-7-boronic acid

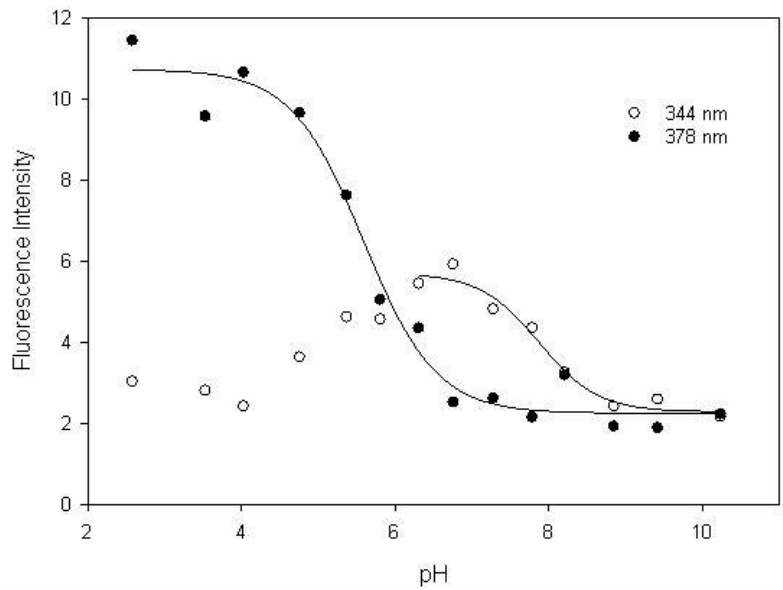

Figure $\mathrm{C} 1$. pH profiles of fluorescent intensities for 7-IQBA $\left(1 \times 10^{-5} \mathrm{M}\right)$ in the absence of sugar in phosphate buffer $(0.1 \mathrm{M}): \lambda_{\mathrm{ex}}=272 \mathrm{~nm}, \lambda_{\mathrm{em}}=344 \mathrm{~nm}$ and $378 \mathrm{~nm}$. 
Isoquinoline-7-boronic acid + D-Fructose

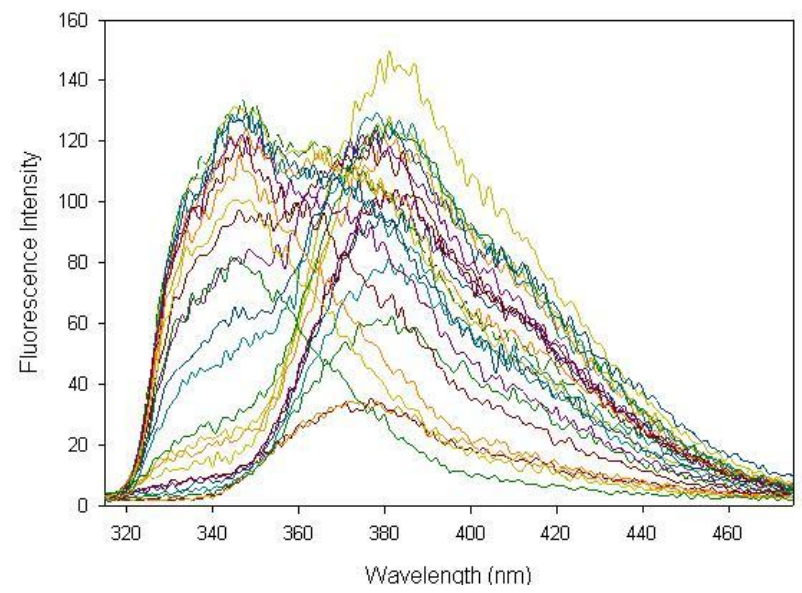

Isoquinoline-7-boronic acid + D-Fructose ${ }^{[16]}$

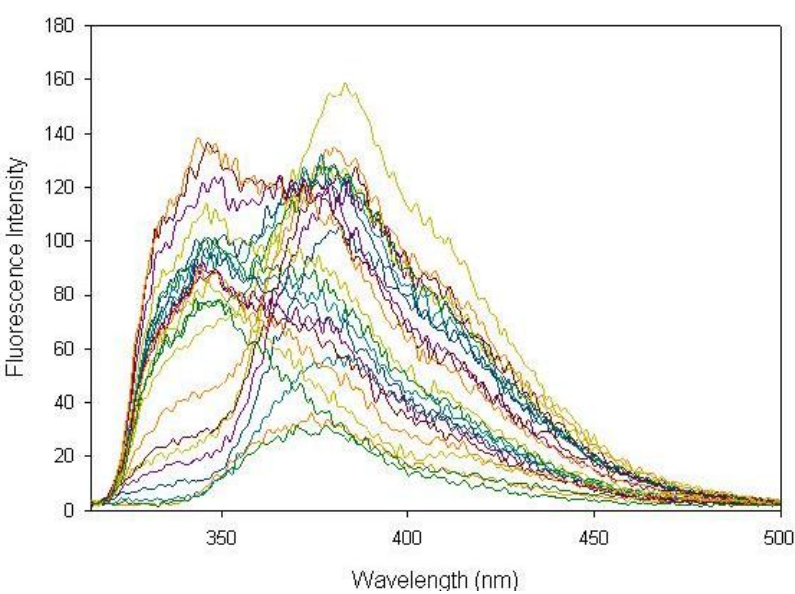

Isoquinoline-7-boronic acid + D-Fructose

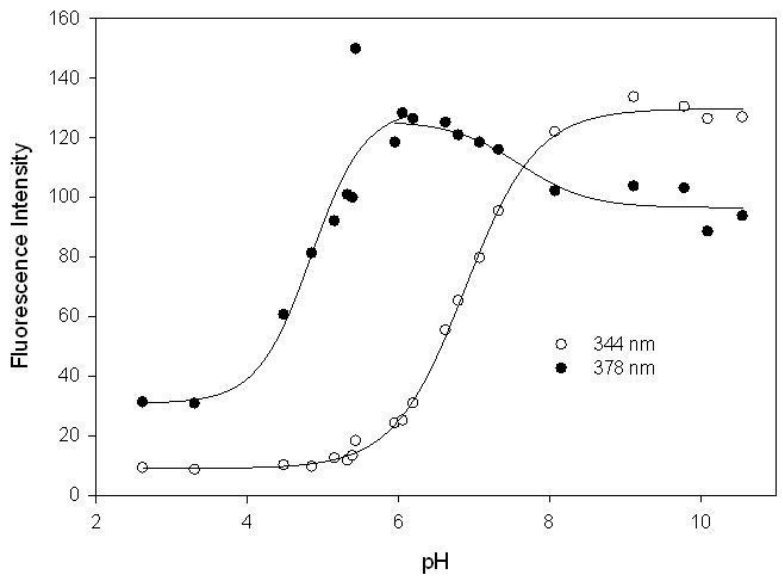

pH Profile of Isoquinoline-7-boronic acid + D-Fructose ${ }^{[16]}$

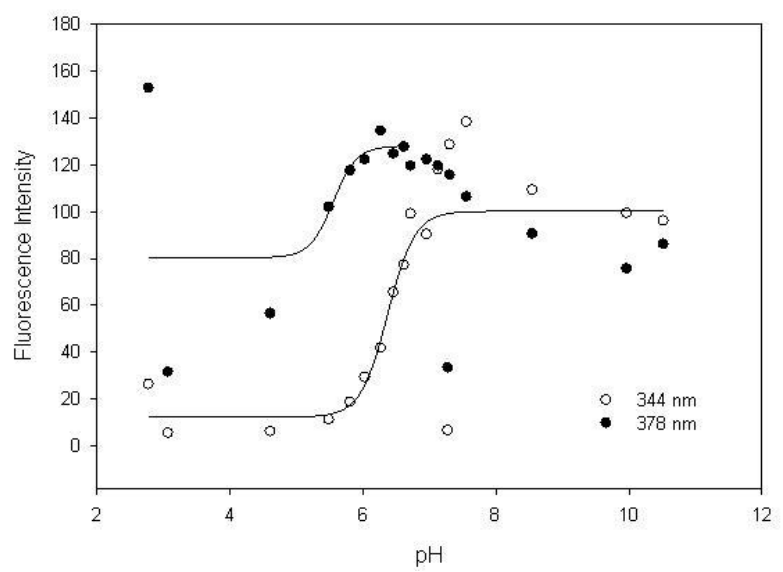

Figure C2. $\mathrm{pH}$ profiles of fluorescent intensities for 7-IQBA $\left(1 \times 10^{-5} \mathrm{M}\right)$ in the presence of Dfructose $(0.01 \mathrm{M})$ in phosphate buffer $(0.1 \mathrm{M}): \lambda_{\mathrm{ex}}=272 \mathrm{~nm}, \lambda_{\mathrm{em}}=344 \mathrm{~nm}$ and $378 \mathrm{~nm}$. 
Isoquinoline-7-boronic acid + D-Mannose

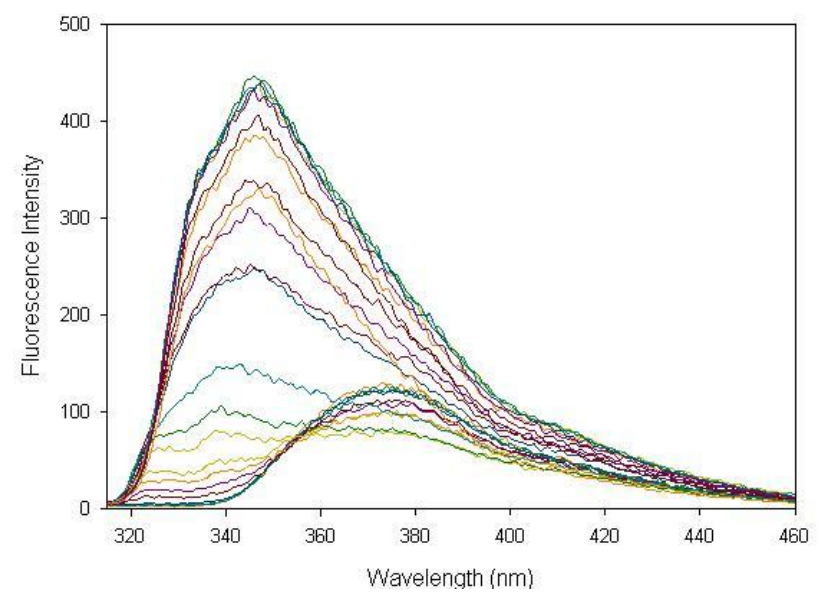

Isoquinoline-7-boronic acid + D-Mannose

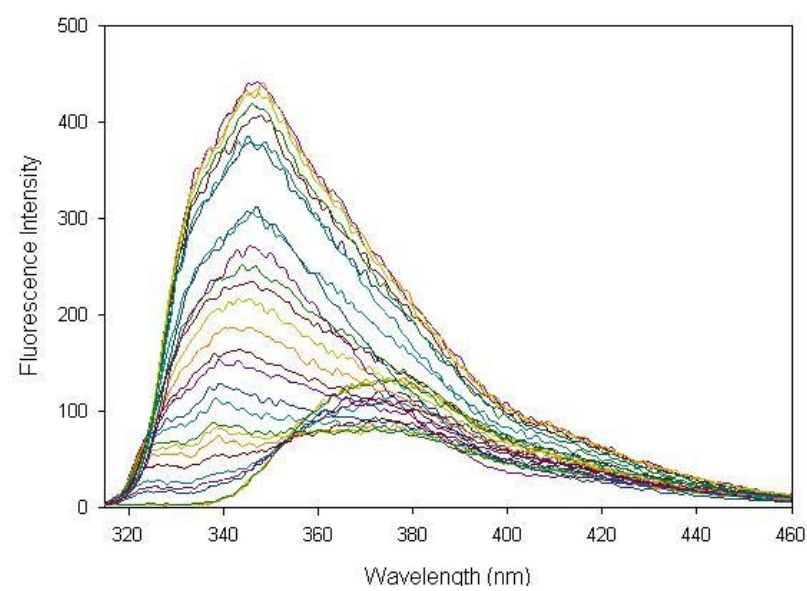

Isoquinoline-7-boronic acid + D-Mannose

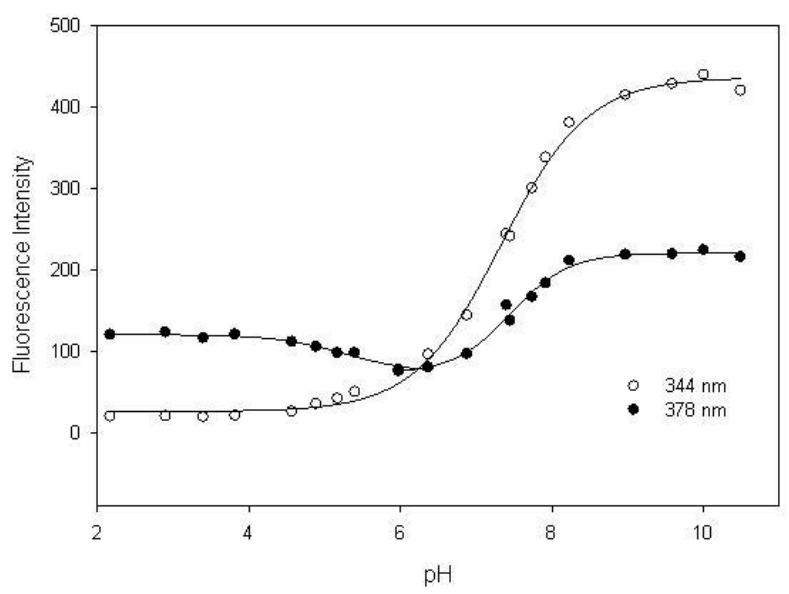

Isoquinoline-7-boronic acid + D-Mannose

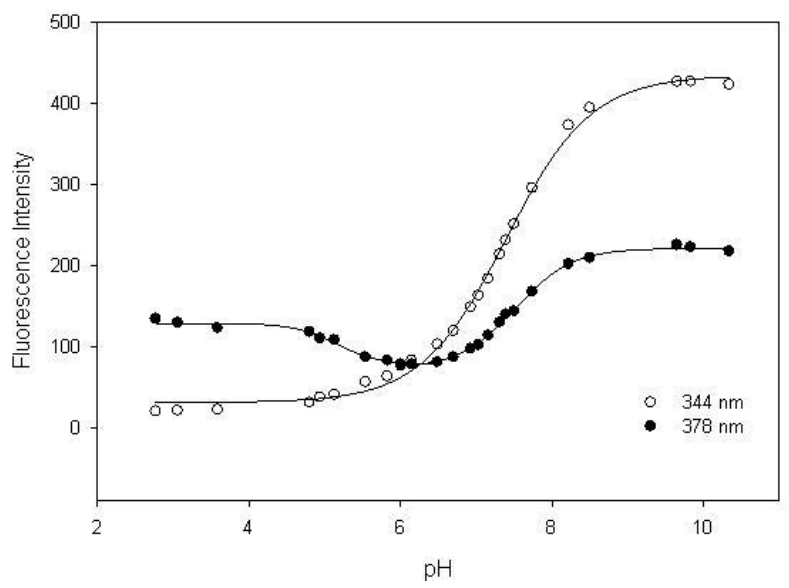

Figure C4. pH profiles of fluorescent intensities for 7-IQBA $\left(1 \times 10^{-5} \mathrm{M}\right)$ in the presence of Dmannose $(0.01 \mathrm{M})$ in phosphate buffer $(0.1 \mathrm{M}): \lambda_{\mathrm{ex}}=271 \mathrm{~nm}, \lambda_{\mathrm{em}}=344 \mathrm{~nm}$ and $378 \mathrm{~nm}$. 
Isoquinoline-7-boronic acid + D-Sorbitol

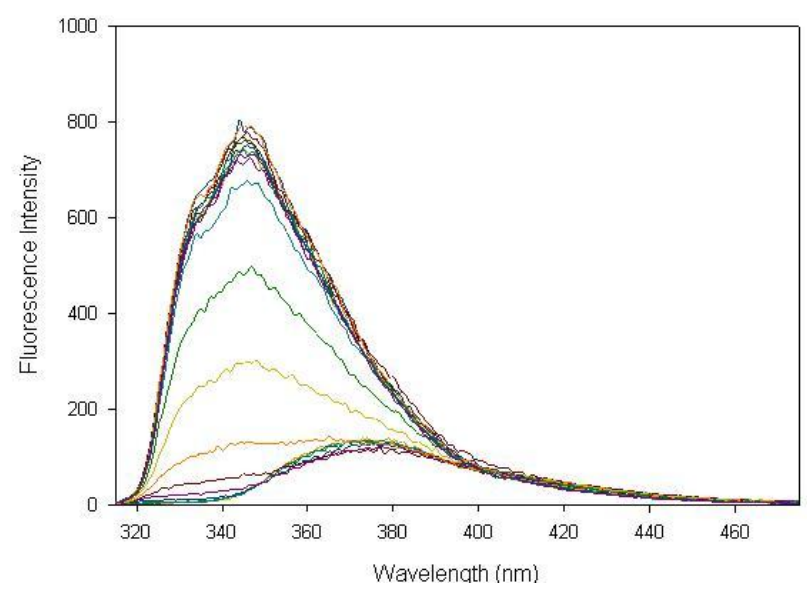

Isoquinoline-7-boronic acid + D-Sorbitol

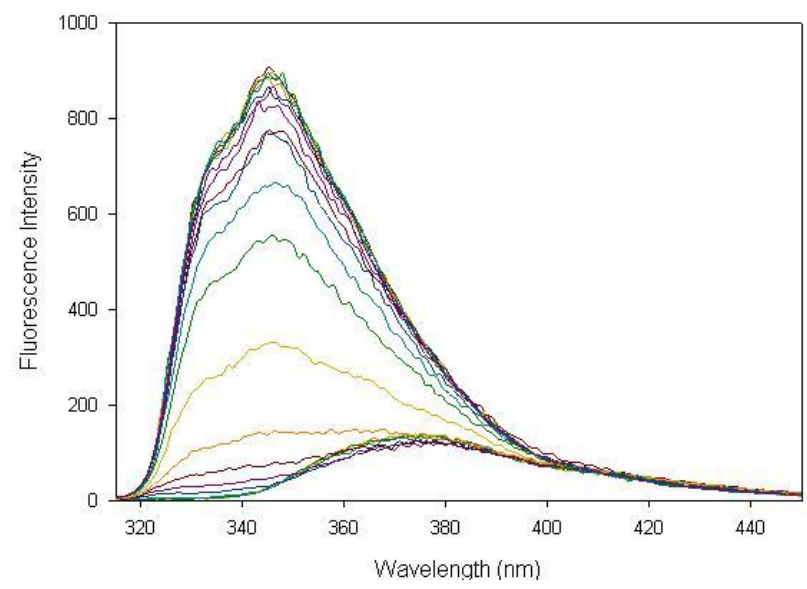

Isoquinoline-7-boronic acid + D-Sorbitol

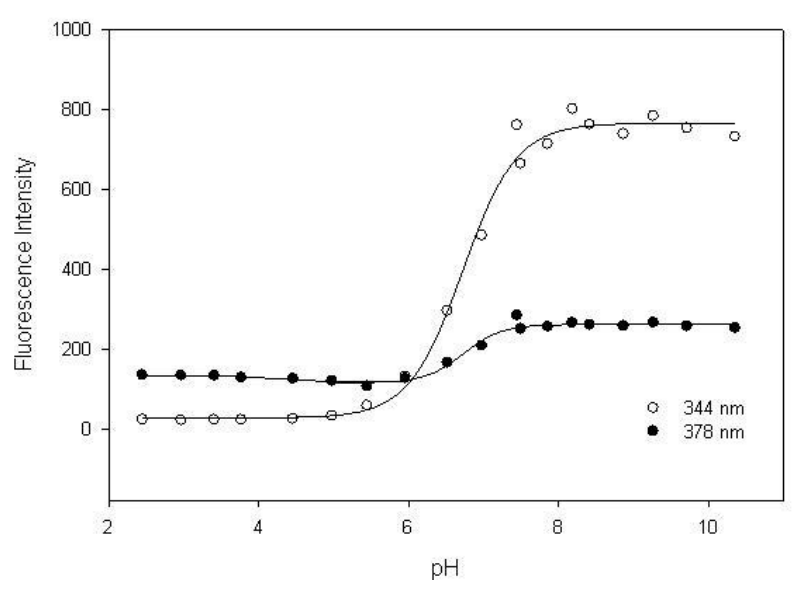

Isoquinoline-7-boronic acid + D-Sorbitol

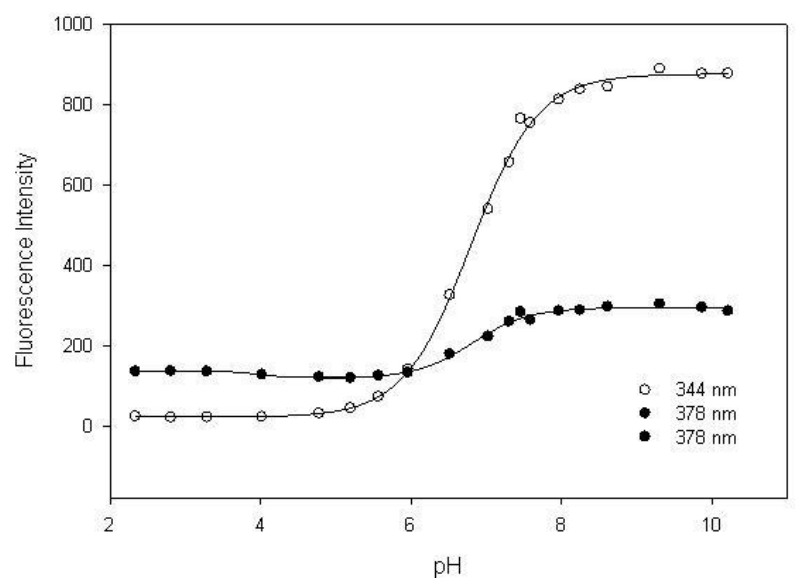

Figure C5. pH profiles of fluorescent intensities for 7-IQBA $\left(1 \times 10^{-5} \mathrm{M}\right)$ in the presence of D-sorbitol $(0.01 \mathrm{M})$ in phosphate buffer $(0.1 \mathrm{M}): \lambda_{\mathrm{ex}}=272 \mathrm{~nm}, \lambda_{\mathrm{em}}=344 \mathrm{~nm}$ and $378 \mathrm{~nm}$. 
Isoquinoline-7-boronic acid + D-Tagatose

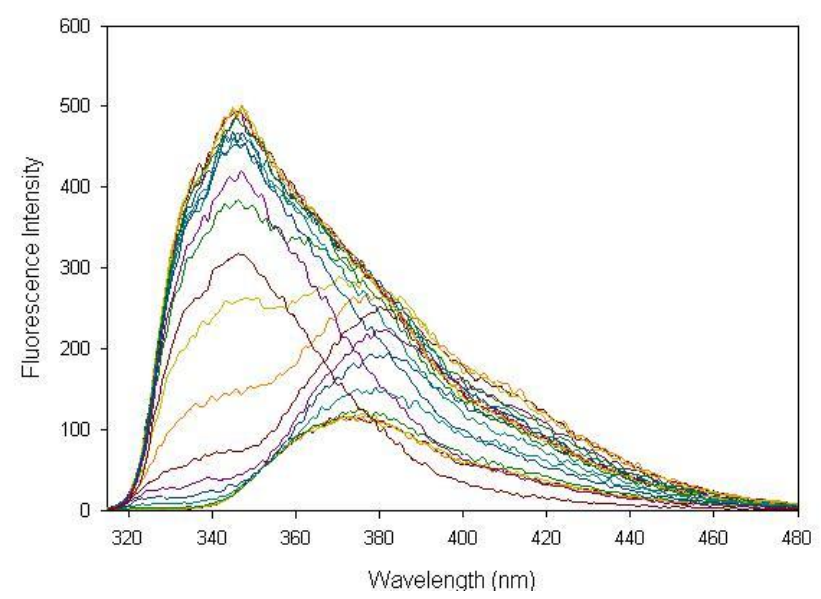

Isoquinoline-7-boronic acid + D-Tagatose

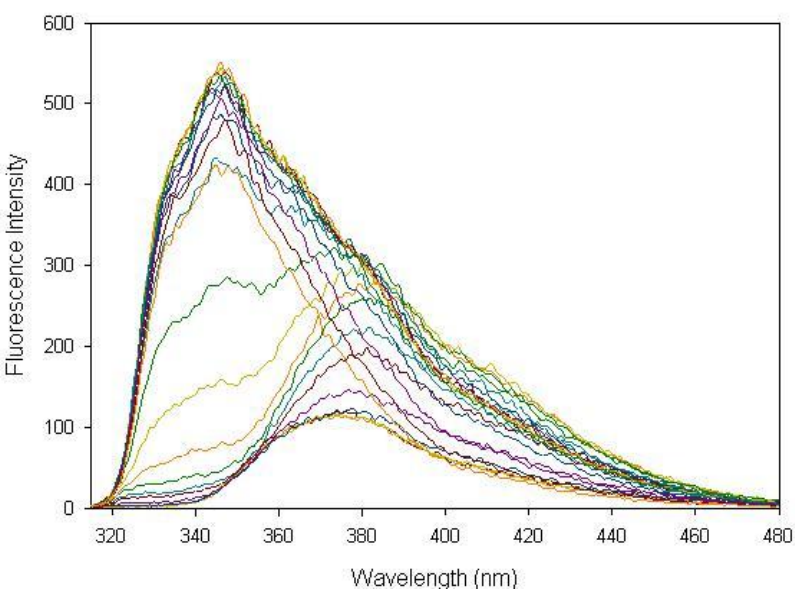

Isoquinoline-7-boronic acid + D-Tagatose

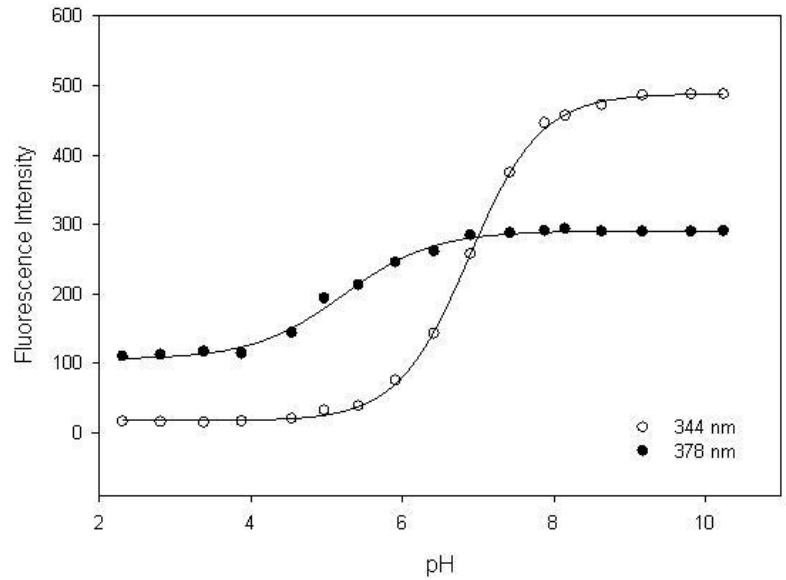

Isoquinoline-7-boronic acid + D-Tagatose

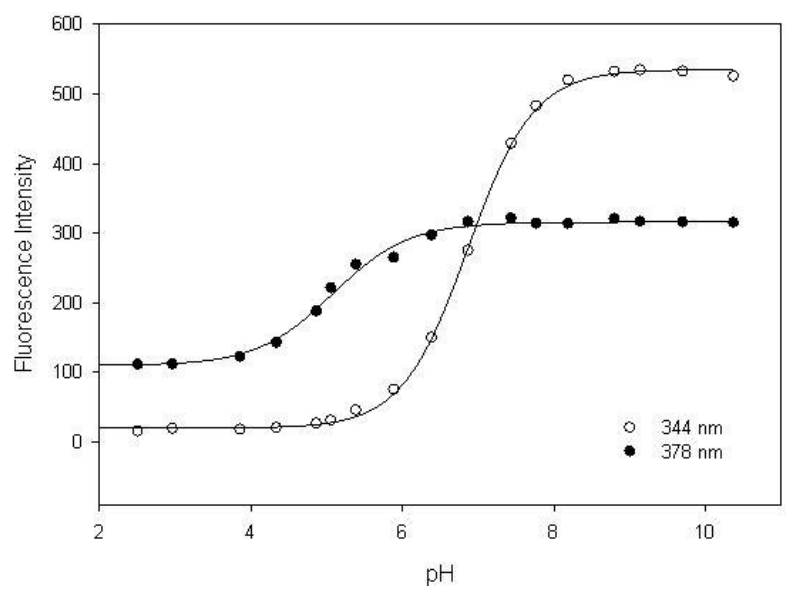

Figure C6. $\mathrm{pH}$ profiles of fluorescent intensities for 7-IQBA $\left(1 \times 10^{-5} \mathrm{M}\right)$ in the presence of Dtagatose $(0.01 \mathrm{M})$ in phosphate buffer $(0.1 \mathrm{M}): \lambda_{\mathrm{ex}}=272 \mathrm{~nm}, \lambda_{\mathrm{em}}=344 \mathrm{~nm}$ and $378 \mathrm{~nm}$. 
Phenoxathiin-4-boronic acid ${ }^{[16]}$

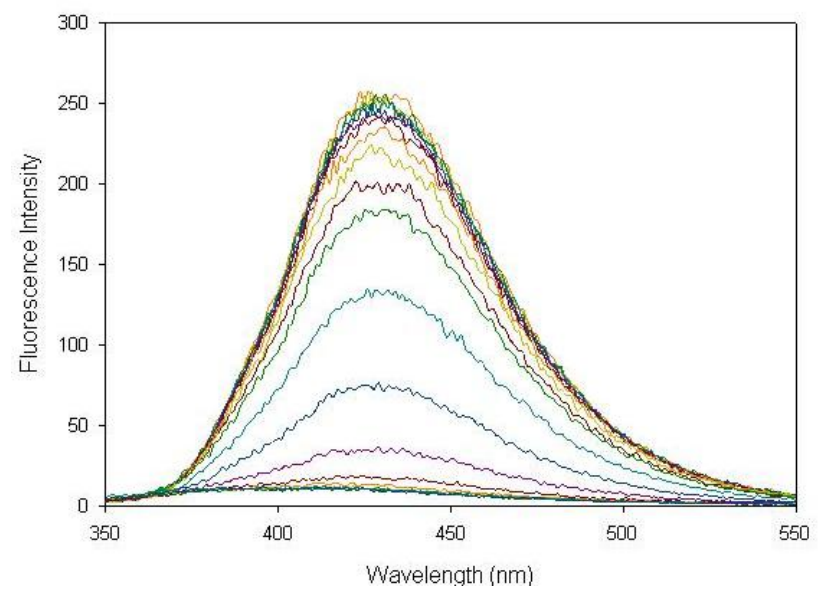

pH Profile of Phenoxathiin-4-boronic acid ${ }^{[16]}$

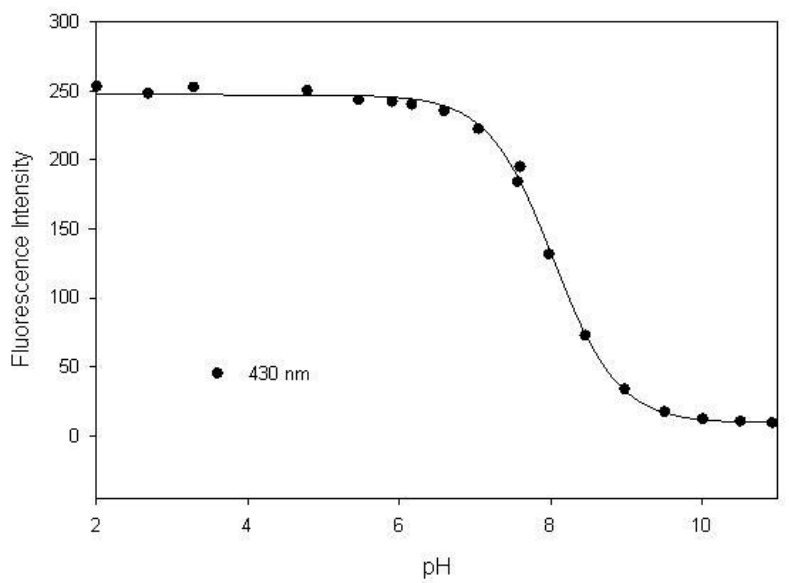

Figure $\mathrm{C7}$. pH profiles of fluorescent intensities for 4-POBA $\left(1 \times 10^{-5} \mathrm{M}\right)$ in the absence of sugar in phosphate buffer $(0.1 \mathrm{M}): \lambda_{\mathrm{ex}}=299 \mathrm{~nm}, \lambda_{\mathrm{em}}=430 \mathrm{~nm}$. 
Phenoxathiin-4-boronic acid + D-Fructose ${ }^{[16]}$

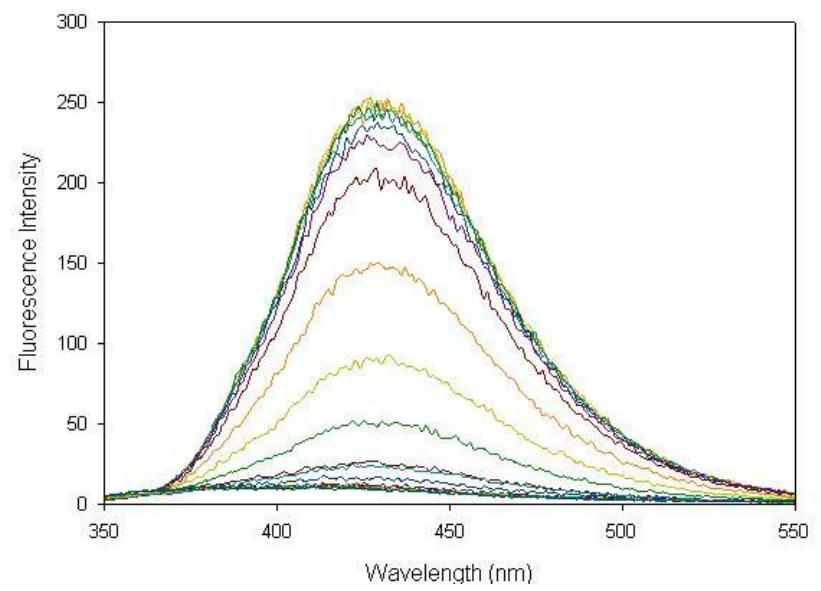

Phenoxathiin-4-boronic acid + D-Fructose ${ }^{[16]}$

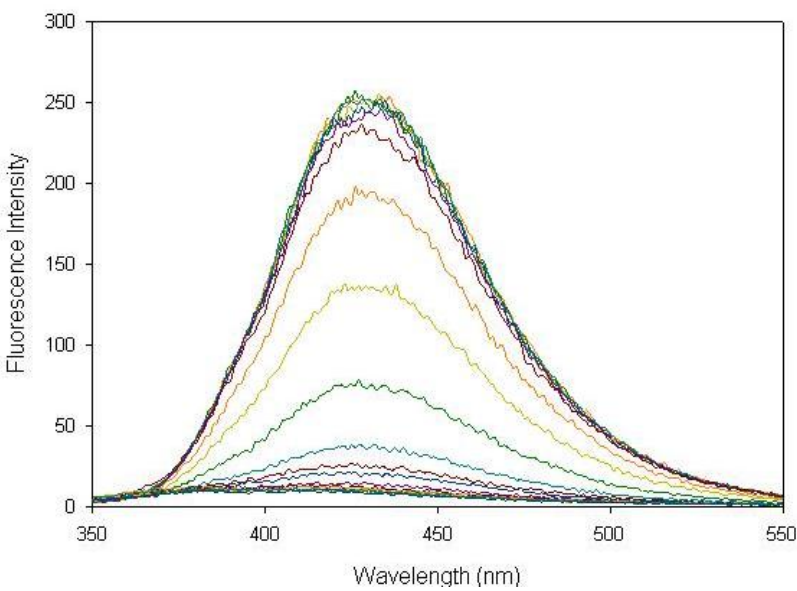

$\mathrm{pH}$ Profile of Phenoxathiin-4-boronic acid + D-Fructose ${ }^{[16]}$

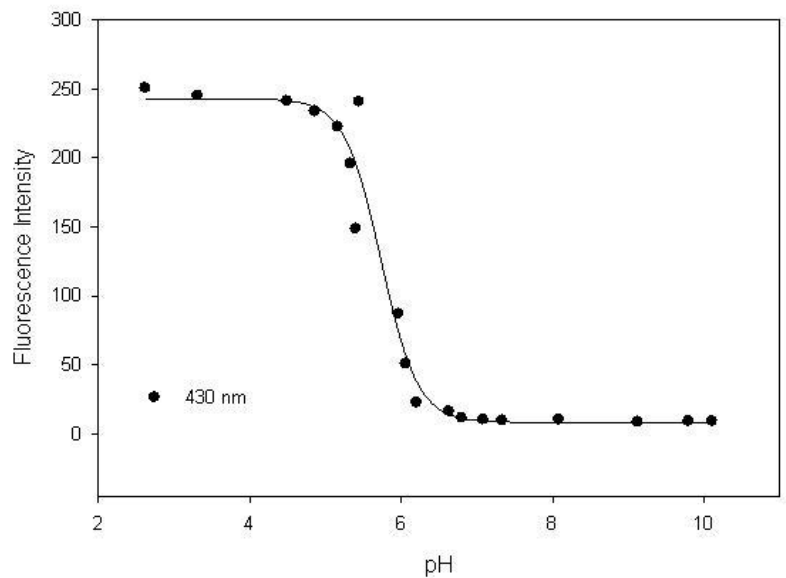

$\mathrm{pH}$ Profile of Phenoxathiin-4-boronic acid + D-Fructose ${ }^{[16]}$

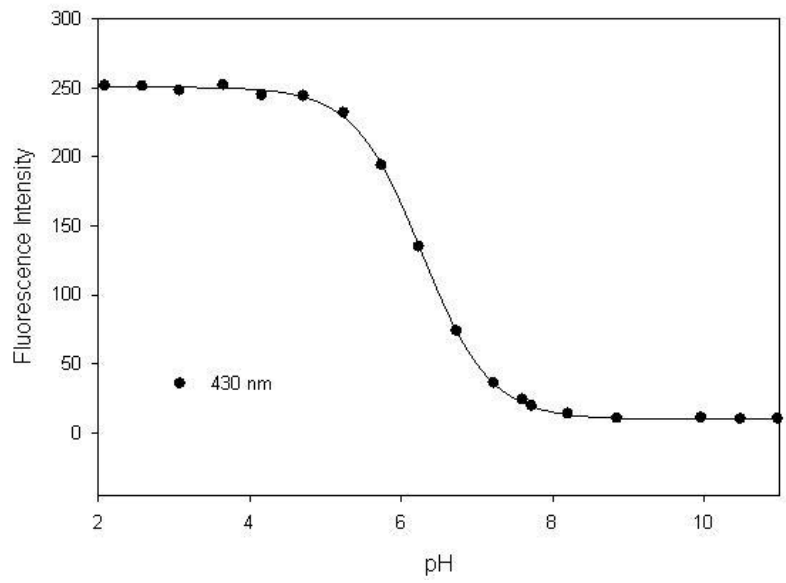

Figure $\mathrm{C} 8$. pH profiles of fluorescent intensities for 4-POBA $\left(1 \times 10^{-5} \mathrm{M}\right)$ in the presence of $\mathrm{D}$ fructose $(0.01 \mathrm{M})$ in phosphate buffer $(0.1 \mathrm{M}): \lambda_{\mathrm{ex}}=299 \mathrm{~nm}, \lambda_{\mathrm{em}}=430 \mathrm{~nm}$. 
Phenoxathiin-4-boronic acid + D-Glucose ${ }^{[16]}$

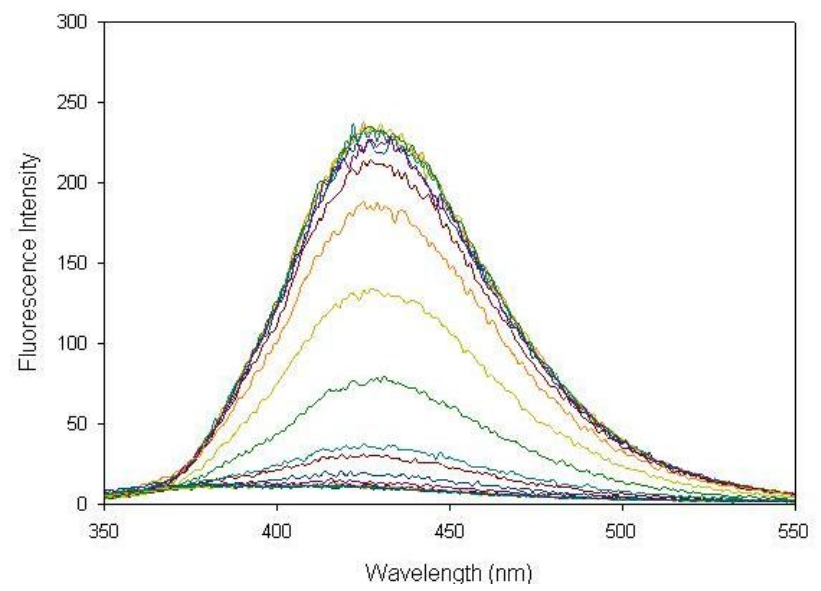

Phenoxathiin-4-boronic acid + D-Glucose ${ }^{[16]}$

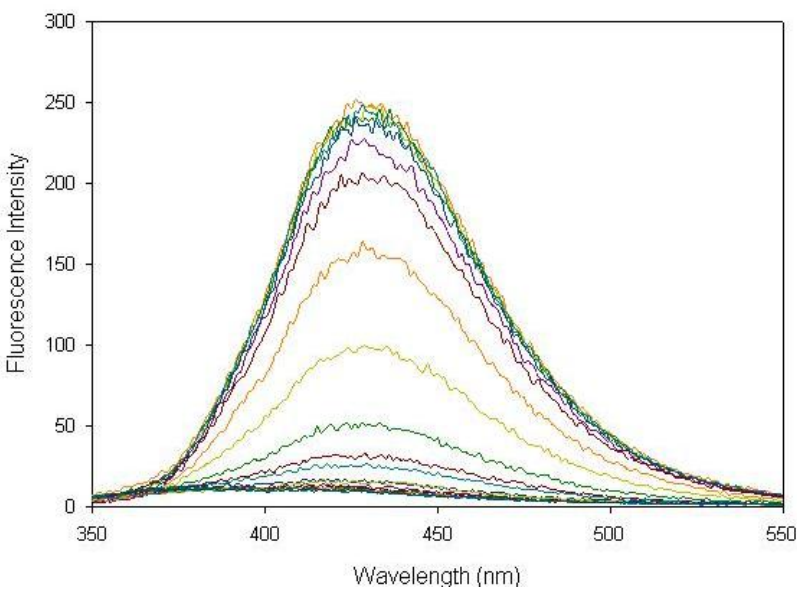

$\mathrm{pH}$ Profile of Phenoxathiin-4-boronic acid + D-Glucose ${ }^{[16]}$

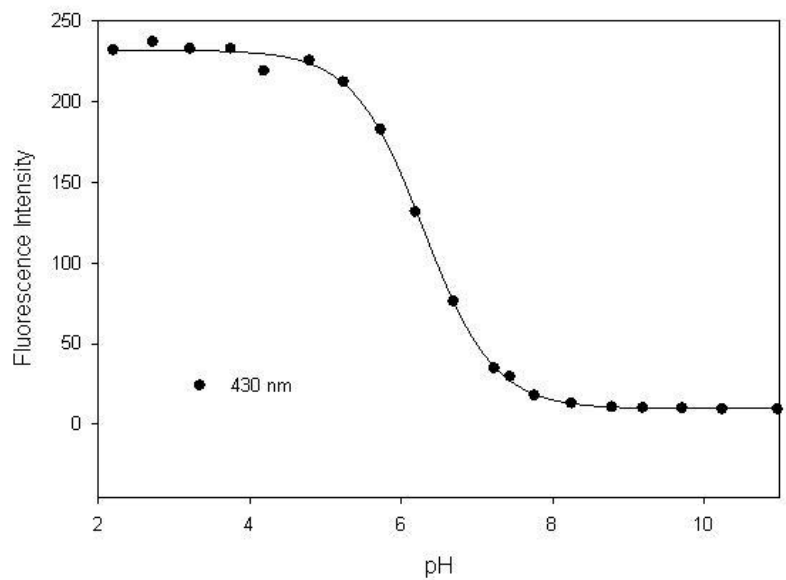

pH Profile of Phenoxathiin-4-boronic acid + D-Glucose ${ }^{[16]}$

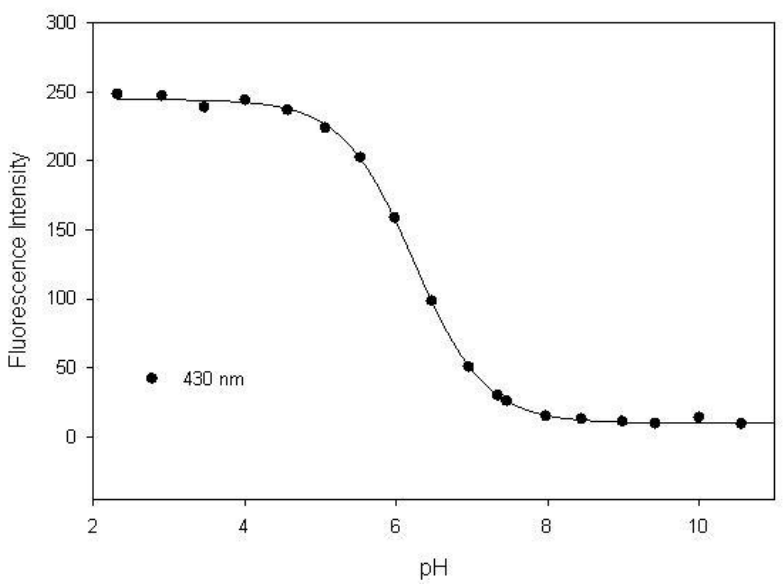

Figure C9. $\mathrm{pH}$ profiles of fluorescent intensities for 4-POBA $\left(1 \times 10^{-5} \mathrm{M}\right)$ in the presence of $\mathrm{D}$ glucose $(0.01 \mathrm{M})$ in phosphate buffer $(0.1 \mathrm{M}): \lambda_{\mathrm{ex}}=299 \mathrm{~nm}, \lambda_{\mathrm{em}}=430 \mathrm{~nm}$. 
Phenoxathiin-4-boronic acid + D-Mannose ${ }^{[16]}$

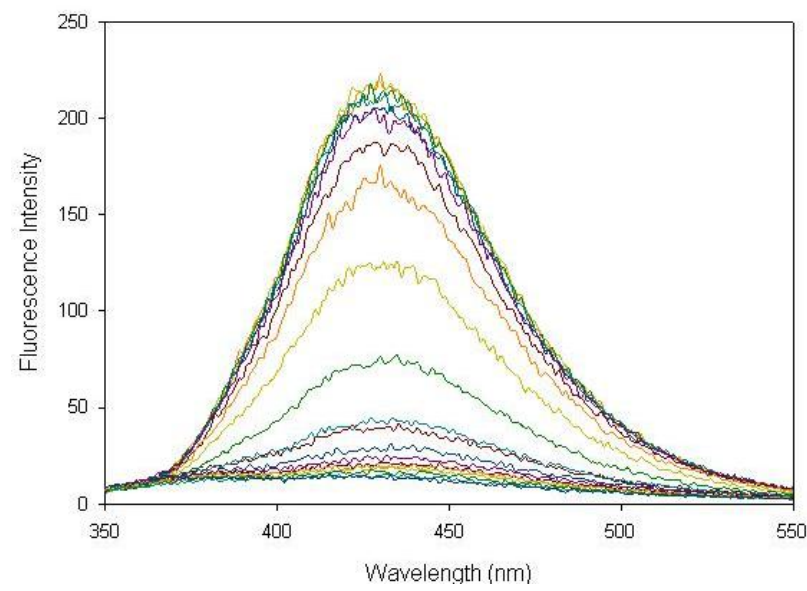

Phenoxathiin-4-boronic acid + D-Mannose ${ }^{[16]}$

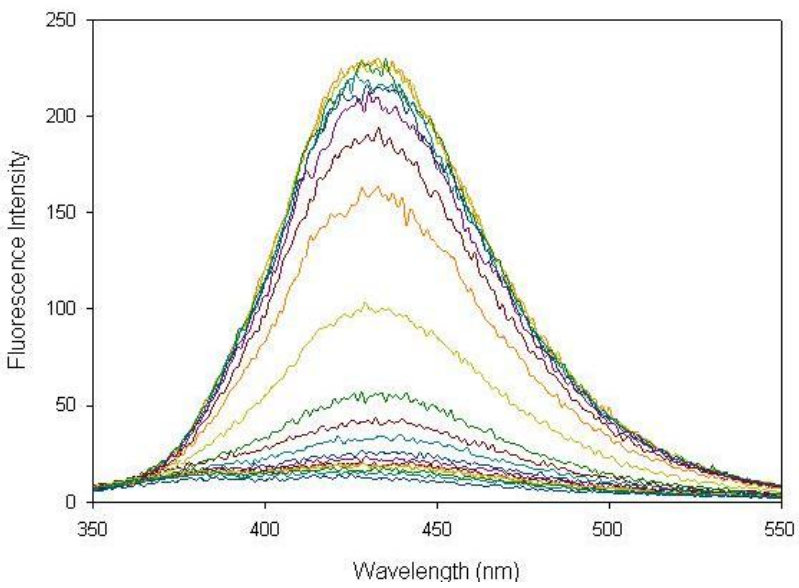

pH Profile of Phenoxathiin-4-boronic acid + D-Mannose ${ }^{[16]}$

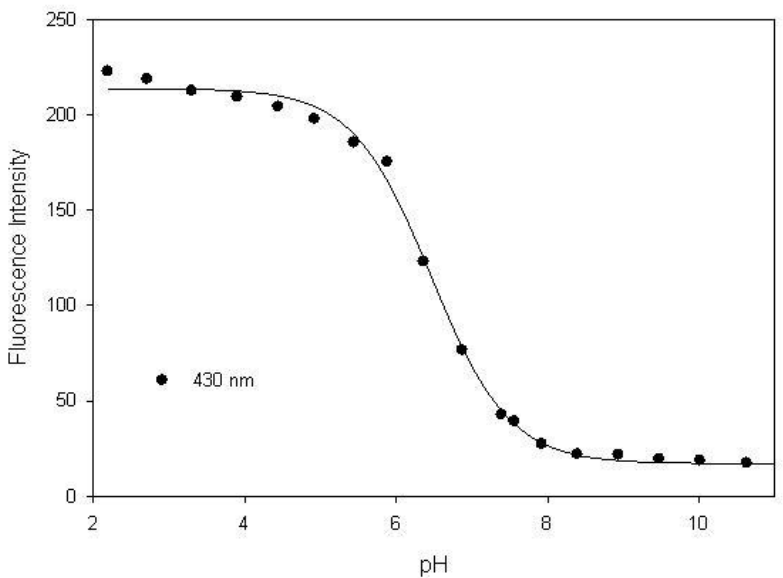

pH Profile of Phenoxathiin-4-boronic acid + D-Mannose ${ }^{[16]}$

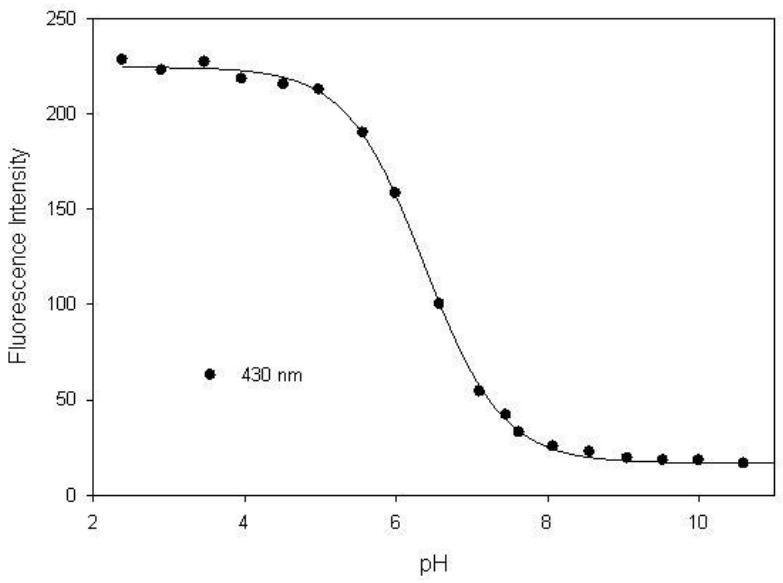

Figure $\mathrm{C} 10 . \mathrm{pH}$ profiles of fluorescent intensities for 4-POBA $\left(1 \times 10^{-5} \mathrm{M}\right)$ in the presence of $\mathrm{D}$ mannose $(0.01 \mathrm{M})$ in phosphate buffer $(0.1 \mathrm{M}): \lambda_{\mathrm{ex}}=299 \mathrm{~nm}, \lambda_{\mathrm{em}}=430 \mathrm{~nm}$. 
Phenoxathiin-4-boronic acid + D-Sorbitol ${ }^{[16]}$

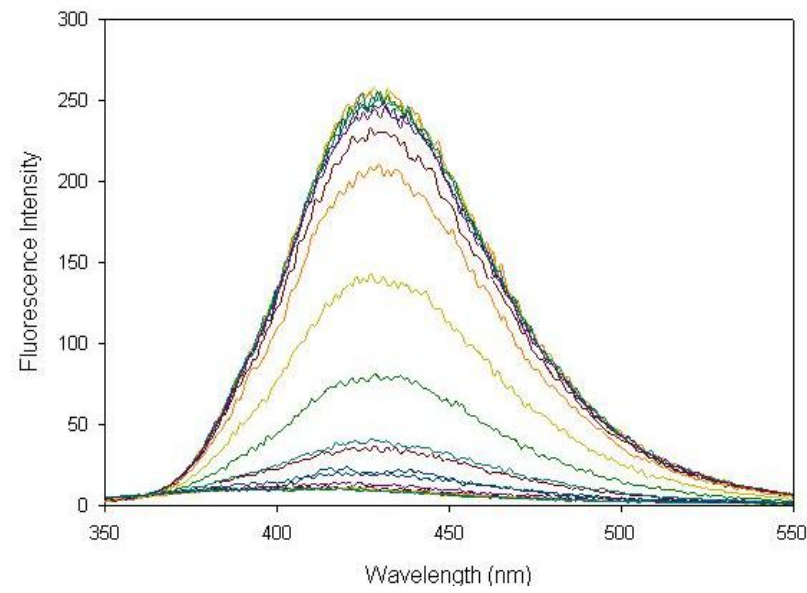

Phenoxathiin-4-boronic acid + D-Sorbitol ${ }^{[16]}$

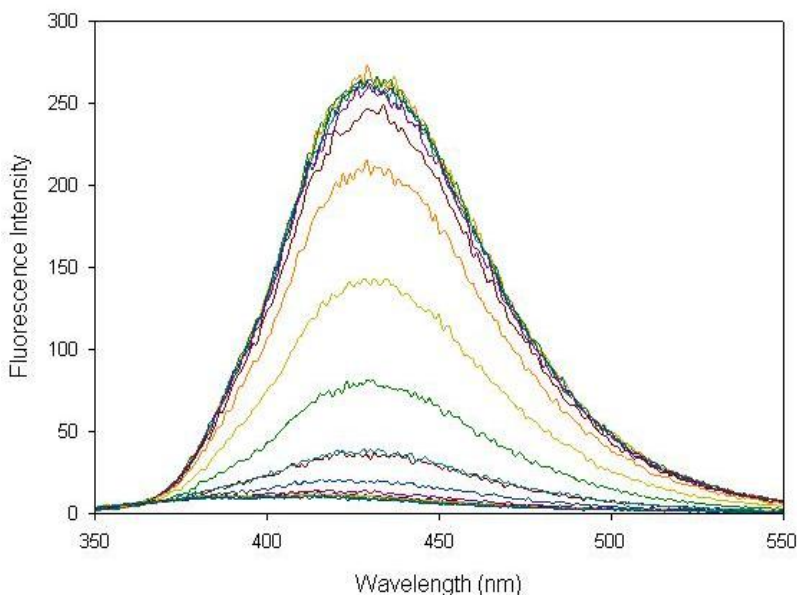

pH Profile of Phenoxathiin-4-boronic acid + D-Sorbitol ${ }^{[16]}$

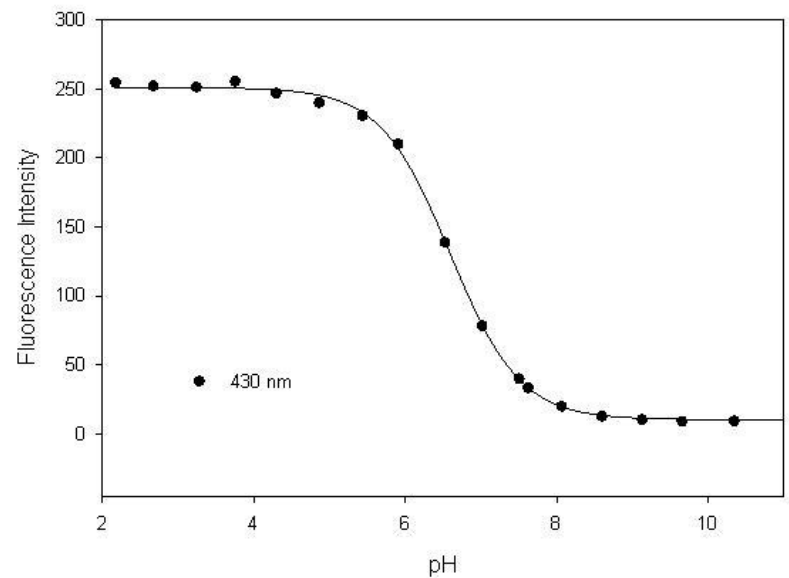

pH Profile of Phenoxathiin-4-boronic acid + D-Sorbitol ${ }^{[16]}$

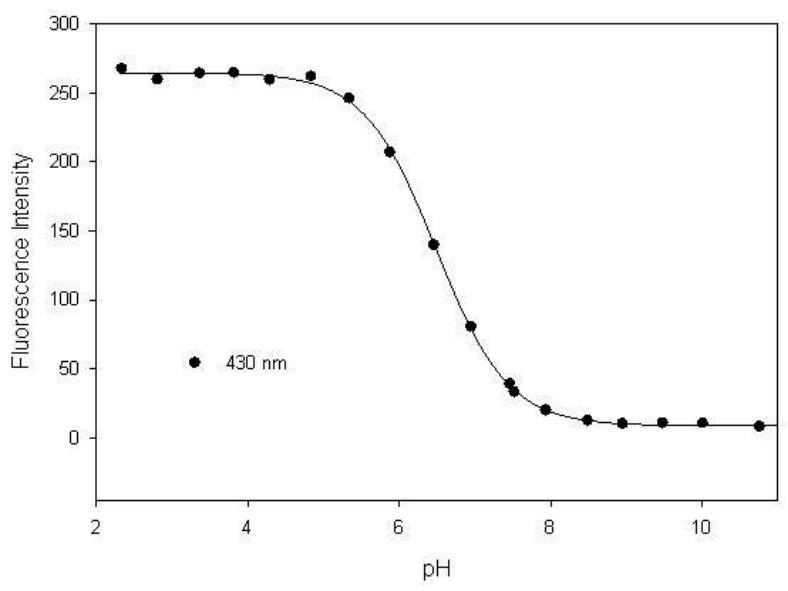

Figure $\mathrm{C} 11$. $\mathrm{pH}$ profiles of fluorescent intensities for 4-POBA $\left(1 \times 10^{-5} \mathrm{M}\right)$ in the presence of $\mathrm{D}$ sorbitol $(0.01 \mathrm{M})$ in phosphate buffer $(0.1 \mathrm{M}): \lambda_{\mathrm{ex}}=299 \mathrm{~nm}, \lambda_{\mathrm{em}}=430 \mathrm{~nm}$. 
Phenoxathiin-4-boronic acid + D-Tagatose ${ }^{[16]}$

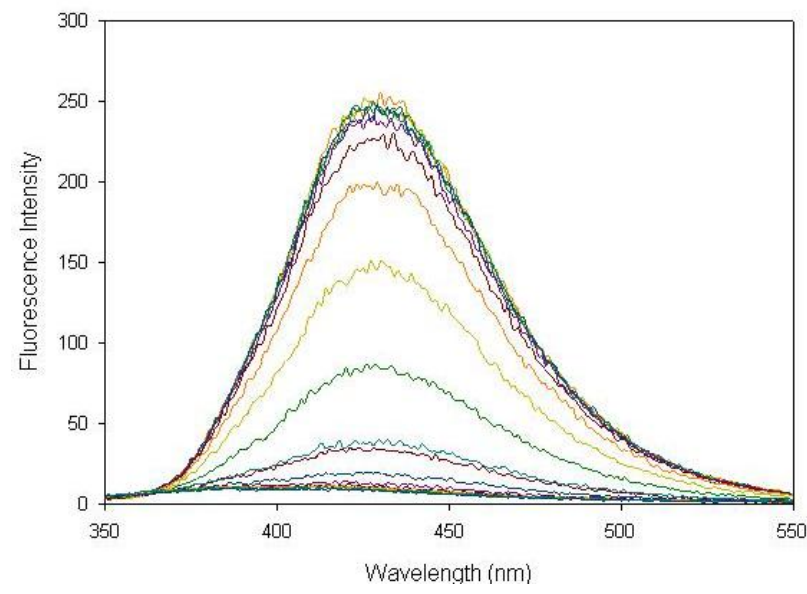

Phenoxathiin-4-boronic acid + D-Tagatose ${ }^{[16]}$

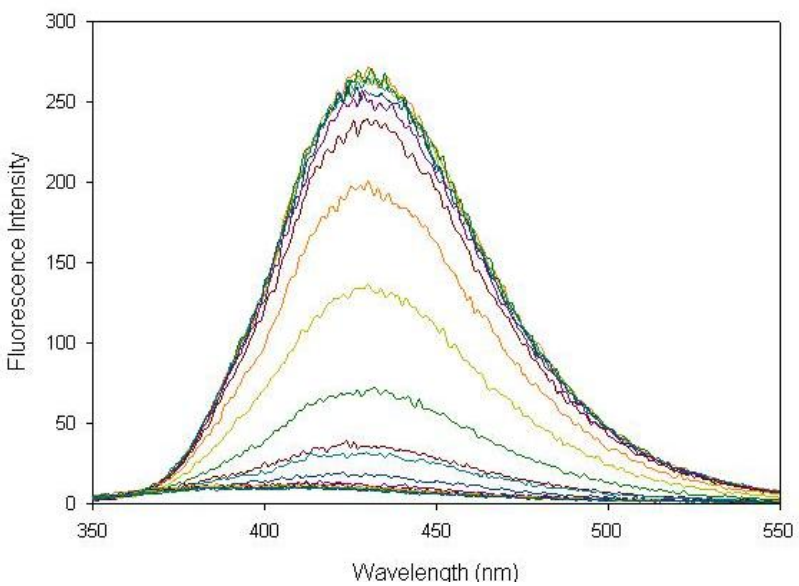

$\mathrm{pH}$ Profile of Phenoxathiin-4-boronic acid + D-Tagatose ${ }^{[16]}$

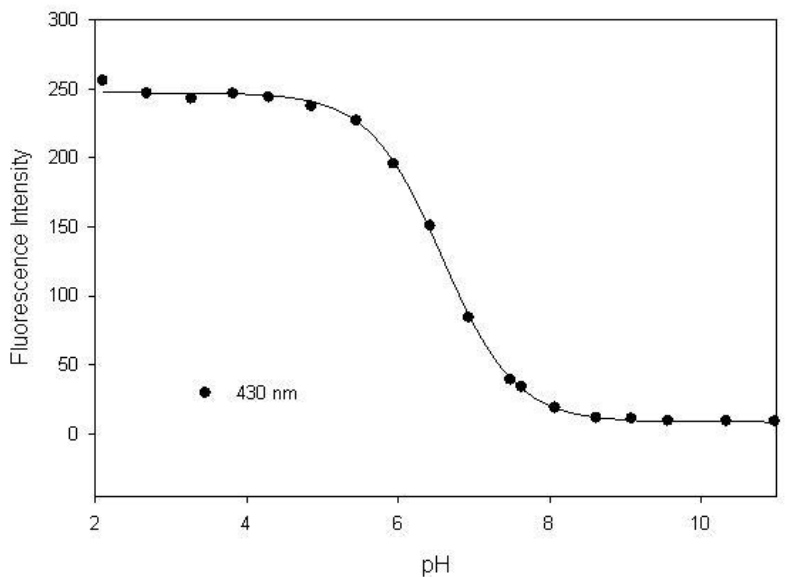

$\mathrm{pH}$ Profile of Phenoxathiin-4-boronic acid + D-Tagatose ${ }^{[16]}$

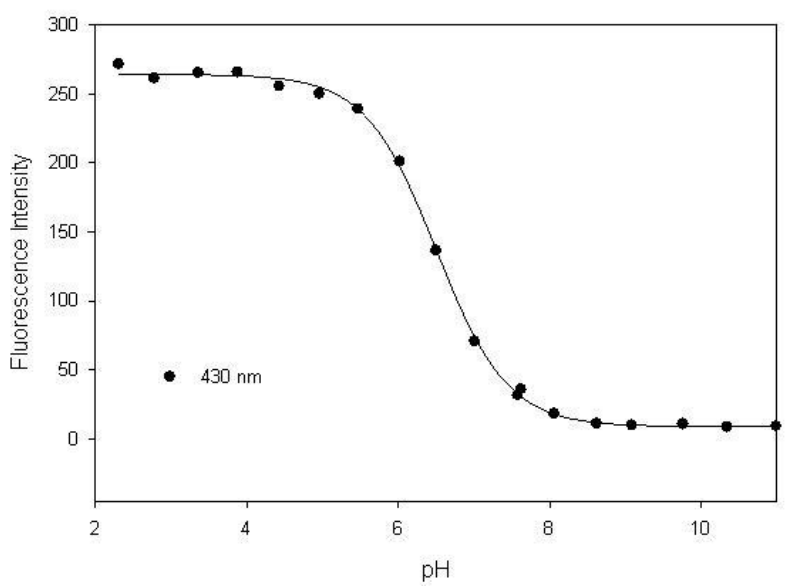

Figure $\mathrm{C12}$. $\mathrm{pH}$ profiles of fluorescent intensities for 4-POBA $\left(1 \times 10^{-5} \mathrm{M}\right)$ in the presence of $\mathrm{D}$ tagatose $(0.01 \mathrm{M})$ in phosphate buffer $(0.1 \mathrm{M}): \lambda_{\mathrm{ex}}=299 \mathrm{~nm}, \lambda_{\mathrm{em}}=430 \mathrm{~nm}$. 
APPENDIX D - Titration Reports for Identification of $\mathrm{p} K_{\mathrm{a}}$ Values

7-IQBA_344nm_1

Nonlinear Regression

Wednesday, September 21, 2011, 11:06:50 AM

Data Source: Data 2 in Titration Reports

Equation: Sigm oidal, Sigmoid, 4 Parameter

$\mathrm{f}=\mathrm{y} 0+\mathrm{a} /(1+\exp (-(\mathrm{x}-\mathrm{x} 0) / \mathrm{b}))$

R Rsqr Adj Rsqr Standard Error of Estimate

$\begin{array}{llll}0.9964 & 0.9929 & 0.9894 & 0.4629\end{array}$

Coefficient Std. Error

$\begin{array}{lrrrr}\text { a } & 10.1206 & 0.4645 & 21.7865 & <0.0001 \\ \text { b } & 0.3003 & 0.0563 & 5.3380 & 0.0018 \\ \text { x0 } & 5.2692 & 0.0511 & 103.1944 & <0.0001 \\ \text { y0 } & 5.4157 & 0.2353 & 23.0206 & <0.0001\end{array}$

Analysis of Variance:

\begin{tabular}{lrrr} 
& DF & \multicolumn{1}{c}{ SS } & \multicolumn{1}{c}{ MS } \\
Regression & 4 & 1084.2156 & 271.0539 \\
Residual & 6 & 1.2856 & 0.2143 \\
Total & 10 & 1085.5012 & 108.5501
\end{tabular}

Corrected for the mean of the observations:

$\begin{array}{llrrrc} & \text { DF } & \text { SS } & \text { MS } & \text { F } & \text { P } \\ \text { Regression } & 3 & 179.9288 & 59.9763 & 279.9163 & <0.0001 \\ \text { Residual } & 6 & 1.2856 & 0.2143 & & \\ \text { Total } & 9 & 181.2143 & 20.1349 & & \end{array}$

\section{Statistical Tests:}

Normality Test (Shapiro-Wilk) $\quad$ Passed $\quad(P=0.1196)$

W Statistic $=0.8767 \quad$ Significance Level $=0.0500$

Constant Variance Test Passed $(\mathrm{P}=0.1988)$

Fit Equation Description:

[Variables]

$\mathrm{x}=\operatorname{col}(1)$

$\mathrm{y}=\operatorname{col}(2)$

reciprocal $\mathrm{y}=1 / \mathrm{abs}(\mathrm{y})$

reciprocal ysquare $=1 / \mathrm{y}^{\wedge} 2$

reciprocal_pred $=1 / \mathrm{abs}(\mathrm{f})$

reciprocal_predsqr $=1 / \mathrm{f}^{\wedge} 2$

[Parameters]

$\mathrm{a}=\max (\mathrm{y})-\min (\mathrm{y}) "$ Auto $\{$ previous: 10.1206$\}\}$

$\mathrm{b}=$ if $(\mathrm{xwtr}(\mathrm{x}, \mathrm{y}-\min (\mathrm{y}), .5)>0, \mathrm{xwtr}(\mathrm{x}, \mathrm{y}-\min (\mathrm{y}), .5) / 4,1)$ "Auto \{\{previous: 0.300289$\}\}$

$\mathrm{x} 0=\mathrm{x} 50(\mathrm{x}, \mathrm{y}-\min (\mathrm{y}), 5)$ "Auto $\{\{$ previous: 5.26924$\}\}$

y0 $=\min (y) "$ Auto $\{\{$ previous: 5.41571$\}\}$

[Equation]

$\mathrm{f}=\mathrm{y} 0+\mathrm{a} /(1+\exp (-(\mathrm{x}-\mathrm{x} 0) / \mathrm{b}))$

fit $\mathrm{f}$ to $\mathrm{y}$ 


\section{7-IQBA_344nm_2}

Nonlinear Regression

Wednesday, September 21, 2011, 11:08:14 AM

Data Source: Data 2 in Titration Reports

Equation: Sigmoidal, Sigmoid, 4 Parameter

$\mathrm{f}=\mathrm{y} 0+\mathrm{a} /(1+\exp (-(\mathrm{x}-\mathrm{x} 0) / \mathrm{b}))$

R Rsqr Adj Rsqr Standard Error of Estimate

$\begin{array}{llll}0.9821 & 0.9645 & 0.9467 & 1.2880\end{array}$

$\begin{array}{llll}\text { Coefficient Std. Error } & t & P\end{array}$

$\begin{array}{lrrrr}\text { a } & 11.3878 & 0.9894 & 11.5100 & <0.0001 \\ \text { b } & -0.1421 & 0.0587 & -2.4204 & 0.0518 \\ \text { x0 } & 7.8091 & 0.0699 & 111.7759 & <0.0001 \\ \text { y0 } & 4.2861 & 0.6568 & 6.5257 & 0.0006\end{array}$

Analysis of Variance:

\begin{tabular}{lrrr} 
& DF & \multicolumn{1}{c}{ SS } & \multicolumn{1}{c}{ MS } \\
Regression & 4 & 1287.8817 & 321.9704 \\
Residual & 6 & 9.9533 & 1.6589 \\
Total & 10 & 1297.8350 & 129.7835
\end{tabular}

Corrected for the mean of the observations:

$\begin{array}{llrrrc} & \text { DF } & \text { SS } & \text { MS } & \text { F } & \text { P } \\ \text { Regression } & 3 & 270.0429 & 90.0143 & 54.2622 & <0.0001 \\ \text { Residual } & 6 & 9.9533 & 1.6589 & & \\ \text { Total } & 9 & 279.9961 & 31.1107 & & \end{array}$

\section{Statistical Tests:}

Normality Test (Shapiro-Wilk) $\quad$ Failed $\quad(\mathrm{P}=0.0145)$

W Statistic $=0.8000 \quad$ Significance Level $=0.0500$

Constant Variance Test Passed $(\mathrm{P}=0.8113)$

Fit Equation Description:

[Variables]

$\mathrm{x}=\operatorname{col}(3)$

$\mathrm{y}=\operatorname{col}(4)$

reciprocal_y $=1 / \operatorname{abs}(\mathrm{y})$

reciprocal_ysquare $=1 / \mathrm{y}^{\wedge} 2$

reciprocal_pred $=1 / \mathrm{abs}(\mathrm{f})$

reciprocal_predsqr $=1 / \mathrm{f}^{\wedge} 2$

[Parameters]

$\mathrm{a}=\max (\mathrm{y})-\min (\mathrm{y})$ "Auto $\{$ previous: 11.3878$\}\}$

$\mathrm{b}=\mathrm{if}(\mathrm{xwtr}(\mathrm{x}, \mathrm{y}-\min (\mathrm{y}), .5) \diamond 0, \mathrm{xwtr}(\mathrm{x}, \mathrm{y}-\min (\mathrm{y}), .5) / 4,1)$ "Auto \{\{previous: -0.142105$\}\}$

$\mathrm{x} 0=\mathrm{x} 50(\mathrm{x}, \mathrm{y}-\min (\mathrm{y}), .5)$ "Auto $\{\{$ previous: 7.8091$\}$ \}

y0 $=\min (y) "$ Auto $\{\{$ previous: 4.28608$\}\}$

[Equation]

$\mathrm{f}=\mathrm{y} 0+\mathrm{a} /(1+\exp (-(\mathrm{x}-\mathrm{x} 0) / \mathrm{b}))$

fit $\mathrm{f}$ to $\mathrm{y}$ 
7-IQBA_344nm_3

Nonlinear Regression

Wednesday, September 21, 2011, 11:26:08 AM

Data Source: Data 3 in Titration Reports

Equation: Sigm oidal, Sigmoid, 4 Parameter

$\mathrm{f}=\mathrm{y} 0+\mathrm{a} /(1+\exp (-(\mathrm{x}-\mathrm{x} 0) / \mathrm{b}))$

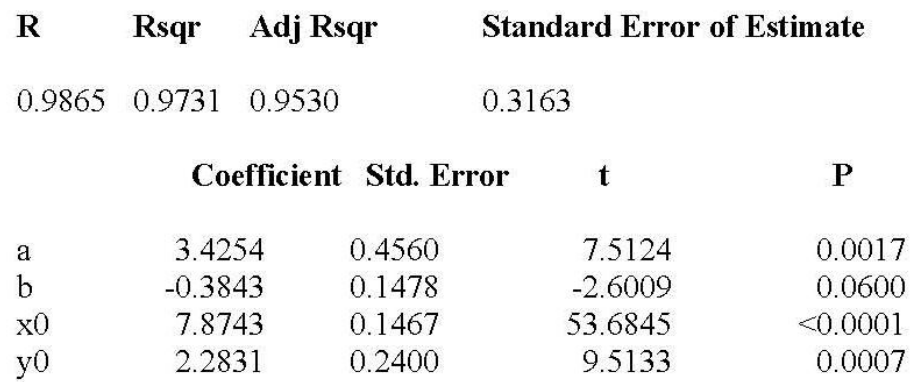

Analysis of Variance:

\begin{tabular}{lrrr} 
& \multicolumn{1}{c}{ DF } & \multicolumn{1}{c}{ SS } & \multicolumn{1}{c}{ MS } \\
Regression & 4 & 134.0835 & 33.5209 \\
Residual & 4 & 0.4003 & 0.1001 \\
Total & 8 & 134.4838 & 16.8105
\end{tabular}

Corrected for the mean of the observations:

$\begin{array}{llcccc} & \text { DF } & \text { SS } & \text { MS } & \text { F } & \text { P } \\ \text { Regression } & 3 & 14.5004 & 4.8335 & 48.3032 & 0.0013 \\ \text { Residual } & 4 & 0.4003 & 0.1001 & & \\ \text { Total } & 7 & 14.9006 & 2.1287 & & \end{array}$

\section{Statistical Tests:}

Normality Test (Shapiro-Wilk)

W Statistic $=0.9329 \quad$ Significance Level $=0.0500$

Constant Variance Test

Passed $(\mathrm{P}=0.1823)$

Fit Equation Description:

[Variables]

$\mathrm{x}=\operatorname{col}(3)$

$\mathrm{y}=\operatorname{col}(4)$

reciprocal $\mathrm{y}=1 / \mathrm{abs}(\mathrm{y})$

reciprocal ysquare $=1 / \mathrm{y}^{\wedge} 2$

reciprocal_pred $=1 / \mathrm{abs}(\mathrm{f})$

reciprocal_predsqr $=1 / \mathrm{f}^{\wedge} 2$

[Parameters]

$\mathrm{a}=\max (\mathrm{y})-\min (\mathrm{y}) "$ Auto $\{$ previous: 3.4254$\}$ \}

$\mathrm{b}=\mathrm{if}(\mathrm{xwtr}(\mathrm{x}, \mathrm{y}-\min (\mathrm{y}), 5) \diamond 0, \operatorname{xwtr}(\mathrm{x}, \mathrm{y}-\min (\mathrm{y}), 5) / 4,1)$ "Auto \{\{previous: -0.384297$\}\}$

$\mathrm{x} 0=\mathrm{x} 50(\mathrm{x}, \mathrm{y}-\min (\mathrm{y}), .5)$ "Auto \{\{previous: 7.87428$\}$ \}

y0 $=\min (y) "$ Auto $\{$ previous: 2.2831$\}\}$

[Equation]

$\mathrm{f}=\mathrm{y} 0+\mathrm{a} /(1+\exp (-(\mathrm{x}-\mathrm{x} 0) / \mathrm{b}))$

fit $\mathrm{f}$ to $\mathrm{y}$ 


\section{7-IQBA_378nm_1}

Nonlinear Regression

Wednesday, September 21, 2011, 11:14:29 AM

Data Source: Data 2 in Titration Reports

Equation: Sigmoidal, Sigmoid, 4 Parameter

$\mathrm{f}=\mathrm{y} 0+\mathrm{a} /(1+\exp (-(\mathrm{x}-\mathrm{x} 0) / \mathrm{b}))$

\begin{tabular}{|c|c|c|c|c|c|}
\hline \multirow{2}{*}{$\begin{array}{l}\mathbf{R} \\
0.9952\end{array}$} & \multirow{2}{*}{ Rsqr } & \multicolumn{2}{|c|}{ Adj Rsqr } & \multicolumn{2}{|c|}{ Standard Error of Estimate } \\
\hline & & 0.9881 & & & \\
\hline \multicolumn{3}{|c|}{ Coefficient } & Std. Error & $\mathbf{t}$ & $\mathbf{P}$ \\
\hline a & \multicolumn{2}{|c|}{22.8653} & 0.9344 & 24.4717 & $<0.0001$ \\
\hline & \multicolumn{2}{|c|}{-0.6784} & 0.0845 & -8.0285 & $<0.0001$ \\
\hline x0 & \multicolumn{2}{|c|}{5.5446} & 0.0883 & 62.8233 & $<0.0001$ \\
\hline yo & \multicolumn{2}{|c|}{3.5108} & 0.4874 & 7.2033 & $<0.0001$ \\
\hline
\end{tabular}

Analysis of Variance:

\begin{tabular}{lrrr} 
& \multicolumn{1}{c}{ DF } & \multicolumn{1}{c}{ SS } & \multicolumn{1}{c}{ MS } \\
Regression 4 & 4046.8964 & 1011.7241 \\
Residual & 13 & 13.5660 & 1.0435 \\
Total & 17 & 4060.4624 & 238.8507
\end{tabular}

Corrected for the mean of the observations:

$\begin{array}{lrrrrr} & \text { DF } & \text { SS } & \text { MS } & \text { F } & \text { P } \\ \text { Regression 3 } & 1389.2712 & 463.0904 & 443.7700 & <0.0001 \\ \text { Residual } 13 & 13.5660 & 1.0435 & & \\ \text { Total } & 16 & 1402.8372 & 87.6773 & & \end{array}$

\section{Statistical Tests:}

Normality Test (Shapiro-Wilk) Passed $\quad(P=0.2549)$

W Statistic $=0.9341 \quad$ Significance Level $=0.0500$

Constant Variance Test Passed $(\mathrm{P}=0.0934)$

Fit Equation Description:

[Variables]

$\mathrm{x}=\operatorname{col}(6)$

$\mathrm{y}=\operatorname{col}(7)$

reciprocal $\mathrm{y}=1 / \mathrm{abs}(\mathrm{y})$

reciprocal_ysquare $=1 / \mathrm{y}^{\wedge} 2$

reciprocal_pred $=1 / \mathrm{abs}(\mathrm{f})$

reciprocal predsqr $=1 / \mathrm{f}^{\wedge} 2$

[Parameters]

$\mathrm{a}=\max (\mathrm{y})-\min (\mathrm{y}) "$ Auto $\{$ previous: 22.8653$\}$ \}

$\mathrm{b}=\mathrm{if}(\mathrm{xwtr}(\mathrm{x}, \mathrm{y}-\min (\mathrm{y}), .5) \diamond 0, \mathrm{xwtr}(\mathrm{x}, \mathrm{y}-\min (\mathrm{y}), .5) / 4,1)$ "Auto \{\{previous: -0.678392$\}\}$

$\mathrm{x} 0=\mathrm{x} 50(\mathrm{x}, \mathrm{y}-\min (\mathrm{y}), .5)$ "Auto \{\{previous: 5.54456$\}\}$

y0 $=\min (y) " A u t o\{\{$ previous: 3.51075$\}\}$

[Equation]

$\mathrm{f}=\mathrm{y} 0+\mathrm{a} /(1+\exp (-(\mathrm{x}-\mathrm{x} 0) / \mathrm{b}))$

fit $\mathrm{f}$ to $\mathrm{y}$ 
7-IQBA_378nm_2

Nonlinear Regression

Wednesday, September 21, 2011, 11:27:48.

Data Source: Data 3 in Titration Reports

Equation: Sigm oidal, Sigmoid, 4 Parameter

$\mathrm{f}=\mathrm{y} 0+\mathrm{a} /(1+\exp (-(\mathrm{x}-\mathrm{x} 0) / \mathrm{b}))$

$\begin{array}{llll}\text { R } & \text { Rsqr } & \text { Adj Rsqr } & \text { Standard Error of Estimate } \\ 0.9896 & 0.9792 & 0.9730 & 0.5983\end{array}$

Coefficient Std. Error t $\quad$ P

$\begin{array}{lrrrr}\text { a } & 8.4982 & 0.5113 & 16.6205 & <0.0001 \\ \text { b } & -0.4714 & 0.1006 & -4.6879 & 0.0009 \\ \text { x0 } & 5.5897 & 0.1103 & 50.6602 & <0.0001 \\ \text { y0 } & 2.2486 & 0.2611 & 8.6108 & <0.0001\end{array}$

Analysis of Variance:

\begin{tabular}{lcrr} 
& \multicolumn{1}{c}{ DF } & \multicolumn{1}{c}{ SS } & \multicolumn{1}{c}{ MS } \\
Regression 4 & 568.6705 & 142.1676 \\
Residual & 10 & 3.5797 & 0.3580 \\
Total & 14 & 572.2502 & 40.8750
\end{tabular}

Corrected for the mean of the observations:

$\begin{array}{lccccc} & \text { DF } & \text { SS } & \text { MS } & \text { F } & \text { P } \\ \text { Regression } 3 & 168.7683 & 56.2561 & 157.1539 & <0.0001 \\ \text { Residual } 10 & 3.5797 & 0.3580 & & \\ \text { Total } & 13 & 172.3480 & 13.2575 & & \end{array}$

\section{Statistical Tests:}

Normality Test (Shapiro-Wilk) Passed $\quad(\mathrm{P}=0.8881)$

W Statistic $=0.9709 \quad$ Significance Level $=0.0500$

Constant Variance Test Passed $(P=0.2311)$

Fit Equation Description:

[Variables]

$\mathrm{x}=\operatorname{col}(5)$

$\mathrm{y}=\operatorname{col}(6)$

reciprocal_y $=1 / a b s(y)$

reciprocal ysquare $=1 / \mathrm{y}^{\wedge} 2$

reciprocal_pred $=1 / \mathrm{abs}(\mathrm{f})$

reciprocal predsqr $=1 / \mathrm{f}^{\wedge} 2$

[Parameters]

$\mathrm{a}=\max (\mathrm{y})-\min (\mathrm{y}) "$ Auto $\{$ \{previous: 8.49823$\}$ \}

$\mathrm{b}=\mathrm{if}(\mathrm{xwtr}(\mathrm{x}, \mathrm{y}-\min (\mathrm{y}), .5)>0, \mathrm{xwtr}(\mathrm{x}, \mathrm{y}-\min (\mathrm{y}), .5) / 4,1)$ "Auto \{ \{previous: -0.471437$\}\}$

$\mathrm{x} 0=\mathrm{x} 50(\mathrm{x}, \mathrm{y}-\mathrm{min}(\mathrm{y}), .5)$ "Auto $\{$ previous: 5.58972$\}\}$

y0 $=\min (y) "$ Auto $\{$ previous: 2.24859$\}$ \}

[Equation]

$\mathrm{f}=\mathrm{y} 0+\mathrm{a} /(1+\exp (-(\mathrm{x}-\mathrm{x} 0) / \mathrm{b}))$

fit $\mathrm{f}$ to $\mathrm{y}$ 


\section{7-IQBA_Fructose_344nm_1}

Nonlinear Regression

Wednesday, September 21, 2011, 12:00:46 PM

Data Source: Data 2 in Fructose Titration

Equation: Sigmoidal, Sigmoid, 4 Parameter

$\mathrm{f}=\mathrm{y} 0+\mathrm{a} /(1+\exp (-(\mathrm{x}-\mathrm{x} 0) / \mathrm{b}))$

$\begin{array}{llll}\mathbf{R} & \text { Rsqr } & \text { Adj Rsqr } & \text { Standard Error of Estimate } \\ 0.9998 & 0.9995 & 0.9994 & 1.2161\end{array}$

Coefficient Std. Error t $\quad$ P

$\begin{array}{lrrrl}\text { a } & 122.5452 & 0.8558 & 143.1989 & <0.0001 \\ \text { b } & 0.4593 & 0.0115 & 39.9326 & <0.0001 \\ \text { x0 } & 6.9139 & 0.0117 & 591.8407 & <0.0001 \\ \text { y0 } & 6.3341 & 0.5320 & 11.9059 & <0.0001\end{array}$

Analysis of Variance:

\begin{tabular}{lrrr} 
& \multicolumn{1}{c}{ DF } & \multicolumn{1}{c}{ SS } & \multicolumn{1}{c}{ MS } \\
Regression 4 & 103723.8963 & 25930.9741 \\
Residual & 16 & 23.6641 & 1.4790 \\
Total & 20 & 103747.5604 & 5187.3780
\end{tabular}

Corrected for the mean of the observations:

$\begin{array}{lrrrrc} & \text { DF } & \text { SS } & \text { MS } & \text { F } & \text { P } \\ \text { Regression } 3 & 48142.7817 & 16047.5939 & 10850.2696 & <0.0001 \\ \text { Residual } & 16 & 23.6641 & 1.4790 & & \\ \text { Total } & 19 & 48166.4458 & 2535.0761 & & \end{array}$

\section{Statistical Tests:}

Normality Test (Shapiro-Wilk) Passed $(\mathrm{P}=0.2891)$

W Statistic $=0.9443 \quad$ Significance Level $=0.0500$

Constant Variance Test $\quad$ Failed $\quad(P=0.0320)$

Fit Equation Description:

[Variables]

$\mathrm{x}=\operatorname{col}(1)$

$\mathrm{y}=\operatorname{col}(2)$

reciprocal_y $=1 / \mathrm{abs}(\mathrm{y})$

reciprocal_ysquare $=1 / \mathrm{y}^{\wedge} 2$

reciprocal_pred $=1 / \mathrm{abs}(\mathrm{f})$

reciprocal_predsqr $=1 / \mathrm{f}^{\wedge} 2$

[Parameters]

$\mathrm{a}=\max (\mathrm{y})-\min (\mathrm{y}) "$ Auto $\{$ previous: 122.545$\}$ \}

$\mathrm{b}=\mathrm{if}(\mathrm{xwtr}(\mathrm{x}, \mathrm{y}-\min (\mathrm{y}), .5)>0, \mathrm{xwtr}(\mathrm{x}, \mathrm{y}-\min (\mathrm{y}), .5) / 4,1)$ "Auto \{\{previous: 0.459337$\}\}$

$\mathrm{x} 0=\mathrm{x} 50(\mathrm{x}, \mathrm{y}-\min (\mathrm{y}), .5)$ "Auto $\{\{$ previous: 6.91393$\}\}$

yo $=\min (y) "$ Auto $\{\{$ previous: 6.3341$\}\}$

[Equation]

$\mathrm{f}=\mathrm{y} 0+\mathrm{a} /(1+\exp (-(\mathrm{x}-\mathrm{x} 0) / \mathrm{b}))$

fit $\mathrm{f}$ to $\mathrm{y}$ 
7-IQBA_Fructose_344nm_2

Nonlinear Regression

Wednesday, September 21, 2011, 12:18:53 PM

Data Source: Data 3 in Fructose Titration

Equation: Sigm oidal, Sigmoid, 4 Parameter

$\mathrm{f}=\mathrm{y} 0+\mathrm{a} /(1+\exp (-(\mathrm{x}-\mathrm{x} 0) / \mathrm{b}))$

$\begin{array}{llll}\text { R } & \text { Rsqr } & \text { Adj Rsqr } & \text { Standard Error of Estimate } \\ 0.9837 & 0.9677 & 0.9602 & 9.4984\end{array}$

Coefficient Std. Error $\quad t \quad$ P

$\begin{array}{lrrrr}\text { a } & 111.7730 & 7.1488 & 15.6352 & <0.0001 \\ \text { b } & 0.3985 & 0.0801 & 4.9724 & 0.0003 \\ \text { x0 } & 6.9083 & 0.0928 & 74.4381 & <0.0001 \\ \text { y0 } & 9.4282 & 4.7674 & 1.9777 & 0.0696\end{array}$

Analysis of Variance:

\begin{tabular}{lrrr} 
& \multicolumn{1}{c}{ DF } & \multicolumn{1}{c}{ SS } & \multicolumn{1}{c}{ MS } \\
Regression 4 & 103964.1648 & 25991.0412 \\
Residual & 13 & 1172.8495 & 90.2192 \\
Total & 17 & 105137.0143 & 6184.5303
\end{tabular}

Corrected for the mean of the observations:

\begin{tabular}{lrrrrc} 
& DF & SS & MS & \multicolumn{1}{c}{ F } & P \\
Regression 3 & 35131.9032 & 11710.6344 & 129.8020 & $<0.0001$ \\
Residual & 13 & 1172.8495 & 90.2192 & & \\
Total & 16 & 36304.7527 & 2269.0470 & &
\end{tabular}

\section{Statistical Tests:}

Normality Test (Shapiro-Wilk) $\quad$ Failed $\quad(\mathrm{P}=0.0491)$

W Statistic $=0.8915 \quad$ Significance Level $=0.0500$

Constant Variance Test Failed $(\mathrm{P}=0.0328)$

Fit Equation Description:

[Variables]

$\mathrm{x}=\operatorname{col}(1)$

$\mathrm{y}=\operatorname{col}(2)$

reciprocal_y $=1 / \operatorname{abs}(\mathrm{y})$

reciprocal_ysquare $=1 / \mathrm{y}^{\wedge} 2$

reciprocal pred $=1 / \mathrm{abs}(\mathrm{f})$

reciprocal_predsqr $=1 / \mathrm{f}^{\wedge} 2$

[Parameters]

$\mathrm{a}=\max (\mathrm{y})-\min (\mathrm{y}) "$ Auto $\{$ previous: 111.773$\}$ \}

$\mathrm{b}=\mathrm{if}(\mathrm{xwtr}(\mathrm{x}, \mathrm{y}-\min (\mathrm{y}), .5)>0, \mathrm{xwtr}(\mathrm{x}, \mathrm{y}-\min (\mathrm{y}), .5) / 4,1)$ "Auto \{\{previous: 0.398512$\}\}$

$\mathrm{x} 0=\mathrm{x} 50(\mathrm{x}, \mathrm{y}-\min (\mathrm{y}), .5)$ "Auto $\{$ previous: 6.90831$\}$ \}

y0 $=\min (\mathrm{y}) "$ Auto $\{\{$ previous: 9.42824$\}\}$

[Equation]

$\mathrm{f}=\mathrm{y} 0+\mathrm{a} /(1+\exp (-(\mathrm{x}-\mathrm{x} 0) / \mathrm{b}))$

fit $\mathrm{f}$ to $\mathrm{y}$ 
7-IQBA_Fructose_378nm_1

Nonlinear Regression

Wednesday, September 21, 2011, 12:00:56 PM

Data Source: Data 2 in Fructose Titration

Equation: Sigm oidal, Sigmoid, 4 Parameter

$\mathrm{f}=\mathrm{y} 0+\mathrm{a} /(1+\exp (-(\mathrm{x}-\mathrm{x} 0) / \mathrm{b}))$

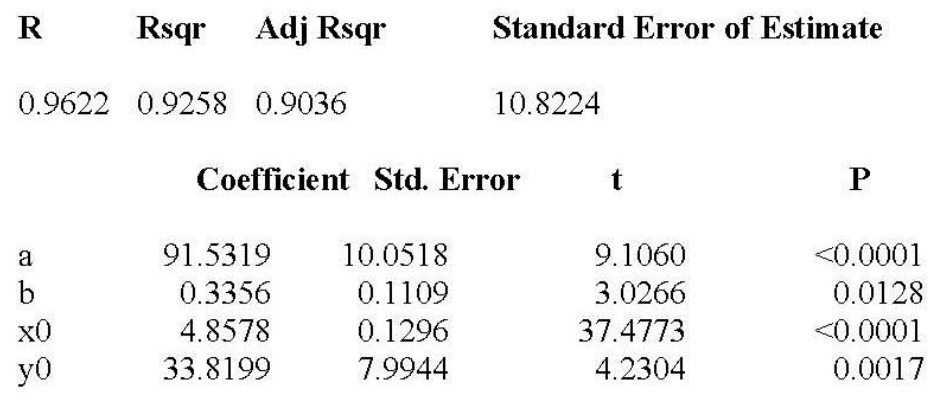

Analysis of Variance:

\begin{tabular}{lrrr} 
& \multicolumn{1}{c}{ DF } & \multicolumn{1}{c}{ SS } & \multicolumn{1}{c}{ MS } \\
Regression 4 & 150054.1496 & 37513.5374 \\
Residual & 10 & 1171.2531 & 117.1253 \\
Total & 14 & 151225.4027 & 10801.8145
\end{tabular}

Corrected for the mean of the observations:

$\begin{array}{lcrrrc} & \text { DF } & \text { SS } & \text { MS } & \text { F } & \text { P } \\ \text { Regression } 3 & 14618.5278 & 4872.8426 & 41.6037 & <0.0001 \\ \text { Residual } & 10 & 1171.2531 & 117.1253 & & \\ \text { Total } & 13 & 15789.7808 & 1214.5985 & & \end{array}$

\section{Statistical Tests:}

Normality Test (Shapiro-Wilk) $\quad$ Failed $\quad(P=0.0016)$

W Statistic $=0.7578 \quad$ Significance Level $=0.0500$

Constant Variance Test Passed $(\mathrm{P}=0.6482)$

Fit Equation Description:

[Variables]

$\mathrm{x}=\operatorname{col}(4)$

$\mathrm{y}=\operatorname{col}(5)$

reciprocal $\mathrm{y}=1 / \mathrm{abs}(\mathrm{y})$

reciprocal ysquare $=1 / \mathrm{y}^{\wedge} 2$

reciprocal pred $=1 / \mathrm{abs}(\mathrm{f})$

reciprocal predsqr $=1 / \mathrm{f}^{\wedge} 2$

[Parameters]

$\mathrm{a}=\max (\mathrm{y})-\min (\mathrm{y}) "$ Auto $\{$ previous: 91.5319$\}$ \}

$\mathrm{b}=\mathrm{if}(\mathrm{xwtr}(\mathrm{x}, \mathrm{y}-\min (\mathrm{y}), .5) \diamond 0, \mathrm{xwtr}(\mathrm{x}, \mathrm{y}-\min (\mathrm{y}), .5) / 4,1)$ "Auto \{\{previous: 0.335641$\}$ \}

$\mathrm{x} 0=\mathrm{x} 50(\mathrm{x}, \mathrm{y}-\min (\mathrm{y}), 5)$ "Auto $\{$ previous: 4.85782$\}$ \}

y0 $=\min (y) "$ Auto $\{\{$ previous: 33.8199$\}$ \}

[Equation]

$\mathrm{f}=\mathrm{y} 0+\mathrm{a} /(1+\exp (-(\mathrm{x}-\mathrm{x} 0) / \mathrm{b}))$

fit $\mathrm{f}$ to $\mathrm{y}$ 
7-IQBA_Fructose_378nm_2

Nonlinear Regression

Wednesday, September 21, 2011, 12:19:18 PM

Data Source: Data 3 in Fructose Titration

Equation: Sigm oidal, Sigmoid, 4 Parameter

$\mathrm{f}=\mathrm{y} 0+\mathrm{a} /(1+\exp (-(\mathrm{x}-\mathrm{x} 0) / \mathrm{b}))$

$\begin{array}{llll}\text { R } & \text { Rsqr } & \text { Adj Rsqr } & \text { Standard Error of Estimate } \\ 0.9678 & 0.9367 & 0.9096 & 12.7260\end{array}$

Coefficient Std. Error $\quad t \quad$ P

$\begin{array}{lrrrr}\text { a } & 97.2885 & 11.2153 & 8.6746 & <0.0001 \\ \text { b } & 0.3222 & 0.1465 & 2.1996 & 0.0638 \\ \text { x0 } & 4.8528 & 0.2367 & 20.5008 & <0.0001 \\ \text { y0 } & 30.3212 & 9.1963 & 3.2971 & 0.0132\end{array}$

Analysis of Variance:

\begin{tabular}{lrrr} 
& \multicolumn{1}{c}{ DF } & \multicolumn{1}{c}{ SS } & \multicolumn{1}{c}{ MS } \\
Regression & 4 & 129600.9988 & 32400.2497 \\
Residual & 7 & 1133.6513 & 161.9502 \\
Total & 11 & 130734.6501 & 11884.9682
\end{tabular}

Corrected for the mean of the observations:

$\begin{array}{lrrrrc} & \text { DF } & \text { SS } & \text { MS } & \text { F } & \text { P } \\ \text { Regression } & 3 & 16780.1424 & 5593.3808 & 34.5377 & 0.0001 \\ \text { Residual } & 7 & 1133.6513 & 161.9502 & & \\ \text { Total } & 10 & 17913.7937 & 1791.3794 & & \end{array}$

\section{Statistical Tests:}

Normality Test (Shapiro-Wilk) $\quad$ Failed $\quad(P=0.0267)$

W Statistic $=0.8344 \quad$ Significance Level $=0.0500$

Constant Variance Test $\quad$ Passed $(P=0.6731)$

Fit Equation Description:

[Variables]

$\mathrm{x}=\operatorname{col}(4)$

$\mathrm{y}=\operatorname{col}(5)$

reciprocal_y $=1 / \operatorname{abs}(\mathrm{y})$

reciprocal ysquare $=1 / \mathrm{y}^{\wedge} 2$

reciprocal_pred $=1 / \mathrm{abs}(\mathrm{f})$

reciprocal predsqr $=1 / \mathrm{f}^{\wedge} 2$

[Parameters]

$\mathrm{a}=\max (\mathrm{y})-\min (\mathrm{y}) "$ Auto $\{$ previous: 97.2885$\}$ \}

$\mathrm{b}=\mathrm{if}(\mathrm{xwtr}(\mathrm{x}, \mathrm{y}-\min (\mathrm{y}), .5)>0, \mathrm{xwtr}(\mathrm{x}, \mathrm{y}-\min (\mathrm{y}), .5) / 4,1)$ "Auto \{\{previous: 0.322204$\}\}$

$\mathrm{x} 0=\mathrm{x} 50(\mathrm{x}, \mathrm{y}-\min (\mathrm{y}), 5)$ "Auto $\{$ previous: 4.85279$\}$ \}

yo $=\min (\mathrm{y}) "$ Auto $\{$ previous: 30.3212$\}\}$

[Equation]

$\mathrm{f}=\mathrm{y} 0+\mathrm{a} /(1+\exp (-(\mathrm{x}-\mathrm{x} 0) / \mathrm{b}))$

fit $\mathrm{f}$ to $\mathrm{y}$ 


\section{7-IQBA_Mannose_344nm_1}

Nonlinear Regression

Wednesday, September 21, 2011, 12:27:46 PM

Data Source: Data 2 in Mannose Titration

Equation: Sigm oidal, Sigmoid, 4 Parameter

$\mathrm{f}=\mathrm{y} 0+\mathrm{a} /(1+\exp (-(\mathrm{x}-\mathrm{x} 0) / \mathrm{b}))$

$\begin{array}{llll}\text { R } & \text { Rsqr } & \text { Adj Rsqr } & \text { Standard Error of Estimate } \\ 0.9987 & 0.9974 & 0.9969 & 9.3090\end{array}$

Coefficient Std. Error t $\quad$ P

$\begin{array}{lrrrr}\text { a } & 411.2727 & 7.1456 & 57.5558 & <0.0001 \\ \mathrm{~b} & 0.5683 & 0.0322 & 17.6508 & <0.0001 \\ \mathrm{x} 0 & 7.3277 & 0.0326 & 224.7619 & <0.0001 \\ \text { y0 } & 25.5070 & 3.6672 & 6.9555 & <0.0001\end{array}$

Analysis of Variance:

\begin{tabular}{lrrr} 
& \multicolumn{1}{c}{ DF } & \multicolumn{1}{c}{ SS } & \multicolumn{1}{c}{ MS } \\
Regression 4 & 1234828.8085 & 308707.2021 \\
Residual & 16 & 1386.5061 & 86.6566 \\
Total & 20 & 1236215.3146 & 61810.7657
\end{tabular}

Corrected for the mean of the observations:

$\begin{array}{lrrrrc} & \text { DF } & \text { SS } & \text { MS } & \text { F } & \text { P } \\ \text { Regression } 3 & 531524.9221 & 177174.9740 & 2044.5634 & <0.0001 \\ \text { Residual } & 16 & 1386.5061 & 86.6566 & & \\ \text { Total } & 19 & 532911.4282 & 28047.9699 & & \end{array}$

\section{Statistical Tests:}

Normality Test (Shapiro-Wilk) Passed $\quad(P=0.7093)$

W Statistic $=0.9679 \quad$ Significance Level $=0.0500$

Constant Variance Test $\quad$ Passed $(P=0.6718)$

Fit Equation Description:

[Variables]

$\mathrm{x}=\operatorname{col}(1)$

$\mathrm{y}=\operatorname{col}(2)$

reciprocal_y $=1 / \mathrm{abs}(\mathrm{y})$

reciprocal ysquare $=1 / \mathrm{y}^{\wedge} 2$

reciprocal pred $=1 / \mathrm{abs}(\mathrm{f})$

reciprocal predsqr $=1 / \mathrm{f}^{\wedge} 2$

[Parameters]

$\mathrm{a}=\max (\mathrm{y})-\min (\mathrm{y}) "$ Auto $\{$ previous: 411.273$\}$ \}

$\mathrm{b}=\mathrm{if}(\mathrm{xwtr}(\mathrm{x}, \mathrm{y}-\min (\mathrm{y}), .5)>0, \mathrm{xwtr}(\mathrm{x}, \mathrm{y}-\min (\mathrm{y}), .5) / 4,1) "$ Auto $\{\{$ previous: 0.568275$\}$ \}

$\mathrm{x} 0=\mathrm{x} 50(\mathrm{x}, \mathrm{y}-\min (\mathrm{y}), .5)$ "Auto $\{$ previous: 7.32768$\}$ \}

y0 $=\min (y) "$ Auto $\{\{$ previous: 25.507$\}\}$

[Equation]

$\mathrm{f}=\mathrm{y} 0+\mathrm{a} /(1+\exp (-(\mathrm{x}-\mathrm{x} 0) / \mathrm{b}))$

fit $\mathrm{f}$ to $\mathrm{y}$ 
7-IQBA_Mannose_344nm_2

Nonlinear Regression

Wednesday, September 21, 2011, 12:36:34 PM

Data Source: Data 1 in Mannose Titration

Equation: Sigm oidal, Sigmoid, 4 Parameter

$\mathrm{f}=\mathrm{y} 0+\mathrm{a} /(1+\exp (-(\mathrm{x}-\mathrm{x} 0) / \mathrm{b}))$

$\begin{array}{llll}\text { R } & \text { Rsqr } & \text { Adj Rsqr } & \text { Standard Error of Estimate } \\ 0.9985 & 0.9970 & 0.9966 & 8.4797\end{array}$

Coefficient Std. Error $\quad t \quad$ P

$\begin{array}{lrrrr}\text { a } & 404.3340 & 6.9148 & 58.4735 & <0.0001 \\ \mathrm{~b} & 0.5604 & 0.0267 & 21.0144 & <0.0001 \\ \mathrm{x} 0 & 7.3929 & 0.0266 & 278.2704 & <0.0001 \\ \text { y0 } & 30.6869 & 3.4728 & 8.8363 & <0.0001\end{array}$

Analysis of Variance:

\begin{tabular}{lrrr} 
& \multicolumn{1}{c}{ DF } & \multicolumn{1}{c}{ SS } & \multicolumn{1}{c}{ MS } \\
Regression & 4 & 1218819.2134 & 304704.8034 \\
Residual & 20 & 1438.1142 & 71.9057 \\
Total & 24 & 1220257.3277 & 50844.0553
\end{tabular}

Corrected for the mean of the observations:

$\begin{array}{lrrrrr} & \text { DF } & \text { SS } & \text { MS } & \text { F } & \text { P } \\ \text { Regression } 3 & 484104.7857 & 161368.2619 & 2244.1647 & <0.0001 \\ \text { Residual } & 20 & 1438.1142 & 71.9057 & & \\ \text { Total } & 23 & 485542.8999 & 21110.5609 & & \end{array}$

\section{Statistical Tests:}

Normality Test (Shapiro-Wilk) Passed $\quad(P=0.1043)$

W Statistic $=0.9313 \quad$ Significance Level $=0.0500$

Constant Variance Test $\quad$ Passed $(P=0.2058)$

Fit Equation Description:

[Variables]

$\mathrm{x}=\operatorname{col}(1)$

$y=\operatorname{col}(2)$

reciprocal_y $=1 / \mathrm{abs}(\mathrm{y})$

reciprocal_ysquare $=1 / \mathrm{y}^{\wedge} 2$

reciprocal_pred $=1 / \mathrm{abs}(\mathrm{f})$

reciprocal_predsqr $=1 / \mathrm{f}^{\wedge} 2$

[Parameters]

$\mathrm{a}=\max (\mathrm{y})-\min (\mathrm{y}) "$ Auto $\{$ previous: 404.334$\}$ \}

$\mathrm{b}=\mathrm{if}(\mathrm{xwtr}(\mathrm{x}, \mathrm{y}-\min (\mathrm{y}), .5)>0, \mathrm{xwtr}(\mathrm{x}, \mathrm{y}-\min (\mathrm{y}), .5) / 4,1)$ "Auto \{\{previous: 0.560362$\}\}$

$\mathrm{x} 0=\mathrm{x} 50(\mathrm{x}, \mathrm{y}-\mathrm{min}(\mathrm{y}), .5)$ "Auto $\{$ previous: 7.39293$\}$ \}

y0 $=\min (y) "$ Auto $\{$ previous: 30.6869$\}$ \}

[Equation]

$\mathrm{f}=\mathrm{y} 0+\mathrm{a} /(1+\exp (-(\mathrm{x}-\mathrm{x} 0) / \mathrm{b}))$

fit $f$ to $y$ 


\section{7-IQBA_Mannose_378nm_1}

Nonlinear Regression

Wednesday, September 21, 2011, 12:27:58 PM

Data Source: Data 2 in Mannose Titration

Equation: Sigm oidal, Sigmoid, 4 Parameter

$\mathrm{f}=\mathrm{y} 0+\mathrm{a} /(1+\exp (-(\mathrm{x}-\mathrm{x} 0) / \mathrm{b}))$

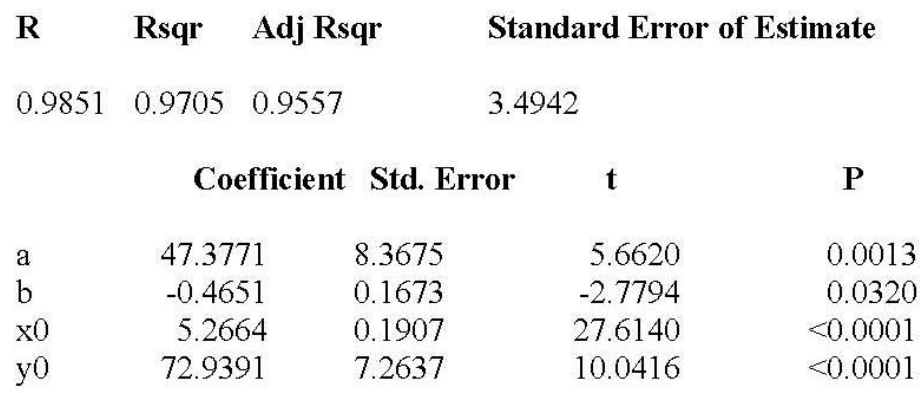

Analysis of Variance:

\begin{tabular}{lrrr} 
& DF & \multicolumn{1}{c}{ SS } & \multicolumn{1}{c}{ MS } \\
Regression & 4 & 111831.8009 & 27957.9502 \\
Residual & 6 & 73.2582 & 12.2097 \\
Total & 10 & 111905.0591 & 11190.5059
\end{tabular}

Corrected for the mean of the observations:

\begin{tabular}{llrrrc} 
& DF & \multicolumn{1}{c}{ SS } & MS & F & P \\
Regression & 3 & 2406.1841 & 802.0614 & 65.6905 & $<0.0001$ \\
Residual & 6 & 73.2582 & 12.2097 & & \\
Total & 9 & 2479.4422 & 275.4936 & &
\end{tabular}

\section{Statistical Tests:}

Normality Test (Shapiro-Wilk)

Passed $(\mathrm{P}=0.7453)$

W Statistic $=0.9565 \quad$ Significance Level $=0.0500$

Constant Variance Test

Passed $(P=0.2274)$

Fit Equation Description:

[Variables]

$\mathrm{x}=\operatorname{col}(4)$

$\mathrm{y}=\operatorname{col}(5)$

reciprocal_y $=1 / \mathrm{abs}(\mathrm{y})$

reciprocal ysquare $=1 / \mathrm{y}^{\wedge} 2$

reciprocal_pred $=1 / \mathrm{abs}(\mathrm{f})$

reciprocal predsqr $=1 / \mathrm{f}^{\wedge} 2$

[Parameters]

$\mathrm{a}=\max (\mathrm{y})-\min (\mathrm{y}) "$ Auto $\{\{$ previous: 47.3771$\}\}$

$\mathrm{b}=\mathrm{if}(\mathrm{xwtr}(\mathrm{x}, \mathrm{y}-\min (\mathrm{y}), .5) \diamond 0, \mathrm{xwtr}(\mathrm{x}, \mathrm{y}-\min (\mathrm{y}), .5) / 4,1)$ "Auto \{\{previous: -0.465073$\}\}$

$\mathrm{x} 0=\mathrm{x} 50(\mathrm{x}, \mathrm{y}-\min (\mathrm{y}), .5)$ "Auto \{\{previous: 5.26638$\}\}$

y0 $=\min (y) "$ Auto $\{\{$ previous: 72.9391$\}$ \}

[Equation]

$\mathrm{f}=\mathrm{y} 0+\mathrm{a} /(1+\exp (-(\mathrm{x}-\mathrm{x} 0) / \mathrm{b}))$

fit $\mathrm{f}$ to $\mathrm{y}$ 


\section{7-IQBA_Mannose_378nm 2}

Nonlinear Regression

Data Source: Data 2 in Mannose Titration

Equation: Sigm oidal, Sigmoid, 4 Parameter

$\mathrm{f}=\mathrm{y} 0+\mathrm{a} /(1+\exp (-(\mathrm{x}-\mathrm{x} 0) / \mathrm{b}))$

$\begin{array}{llll}0.9931 & 0.9862 & 0.9810 & 7.7687\end{array}$

Coefficient Std. Error

$$
\begin{array}{r}
148.2224 \\
0.3808 \\
7.4585
\end{array}
$$

$\begin{array}{lrr}\text { x0 } & 7.4585 & 0.0696 \\ \text { y0 } & 72.4580 & 7.7414\end{array}$

9.4948

0.0672

$\begin{array}{lrr}\mathrm{x} 0 & 7.4585 & 0.0696 \\ \mathrm{y} 0 & 72.4580 & 7.7414\end{array}$

$\begin{array}{lrr}\text { x0 } & 7.4585 & 0.0696 \\ \text { y0 } & 72.4580 & 7.7414\end{array}$

Analysis of Variance:

\begin{tabular}{lrrr} 
& DF & \multicolumn{1}{c}{ SS } & \multicolumn{1}{c}{ MS } \\
Regression & 4 & 362409.5753 & 90602.3938 \\
Residual & 8 & 482.8203 & 60.3525 \\
Total & 12 & 362892.3955 & 30241.0330
\end{tabular}

Corrected for the mean of the observations:

\begin{tabular}{lrrrrr} 
& DF & \multicolumn{1}{c}{ SS } & MS & \multicolumn{1}{c}{$\mathbf{F}$} & \multicolumn{1}{c}{ P } \\
Regression & 3 & 34476.8546 & 11492.2849 & 190.4193 & $<0.0001$ \\
Residual & 8 & 482.8203 & 60.3525 & & \\
Total & 11 & 34959.6749 & 3178.1523 & &
\end{tabular}

Statistical Tests:

Normality Test (Shapiro-Wilk)

Passed $\quad(P=0.3635)$

W Statistic $=0.9284 \quad$ Significance Level $=0.0500$

\section{Constant Variance Test $\quad$ Passed $(P=0.8690)$}

Fit Equation Description:

[Variables]

$\mathrm{x}=\operatorname{col}(6)$

$\mathrm{y}=\operatorname{col}(7)$

reciprocal_y $=1 / \mathrm{abs}(\mathrm{y})$

reciprocal ysquare $=1 / \mathrm{y}^{\wedge} 2$

reciprocal pred $=1 / \mathrm{abs}(\mathrm{f})$

reciprocal_predsqr $=1 / \mathrm{f}^{\wedge} 2$

[Parameters]

$\mathrm{a}=\max (\mathrm{y})-\min (\mathrm{y}) "$ Auto $\{$ previous: 148.222$\}$ \}

$\mathrm{b}=\mathrm{if}(\mathrm{xwtr}(\mathrm{x}, \mathrm{y}-\min (\mathrm{y}), .5)<0, \mathrm{xwtr}(\mathrm{x}, \mathrm{y}-\min (\mathrm{y}), .5) / 4,1) "$ Auto $\{\{$ previous: 0.380833$\}$ \}

$\mathrm{x} 0=\mathrm{x} 50(\mathrm{x}, \mathrm{y}-\min (\mathrm{y}), .5)$ "Auto $\{$ previous: 7.45846$\}$ \}

y0 $=\min (y) "$ Auto $\{\{$ previous: 72.458$\}\}$

[Equation]

$\mathrm{f}=\mathrm{y} 0+\mathrm{a} /(1+\exp (-(\mathrm{x}-\mathrm{x} 0) / \mathrm{b}))$

fit $\mathrm{f}$ to $\mathrm{y}$ 
7-IQBA_Mannose_378nm_3

Nonlinear Regression

Wednesday, September 21, 2011, 12:36:46 PM

Data Source: Data 1 in Mannose Titration

Equation: Sigm oidal, Sigmoid, 4 Parameter

$\mathrm{f}=\mathrm{y} 0+\mathrm{a} /(1+\exp (-(\mathrm{x}-\mathrm{x} 0) / \mathrm{b}))$

\section{R Rsqr Adj Rsqr Standard Error of Estimate \\ $\begin{array}{llll}0.9895 & 0.9791 & 0.9702 & 3.7770\end{array}$}

Coefficient Std. Error $\quad t \quad$ P

$\begin{array}{lrrrr}\text { a } & 51.2683 & 3.9593 & 12.9489 & <0.0001 \\ \text { b } & -0.2836 & 0.0719 & -3.9440 & 0.0056 \\ \text { x0 } & 5.1598 & 0.0783 & 65.9234 & <0.0001 \\ \text { y0 } & 77.2766 & 3.0584 & 25.2673 & <0.0001\end{array}$

Analysis of Variance:

\begin{tabular}{lrrr} 
& \multicolumn{1}{c}{ DF } & \multicolumn{1}{c}{ SS } & \multicolumn{1}{c}{ MS } \\
Regression & 4 & 119771.1654 & 29942.7914 \\
Residual & 7 & 99.8579 & 14.2654 \\
Total & 11 & 119871.0233 & 10897.3658
\end{tabular}

Corrected for the mean of the observations:

$\begin{array}{lrrrrc} & \text { DF } & \text { SS } & \text { MS } & \text { F } & \text { P } \\ \text { Regression } & 3 & 4681.6177 & 1560.5392 & 109.3932 & <0.0001 \\ \text { Residual } & 7 & 99.8579 & 14.2654 & & \\ \text { Total } & 10 & 4781.4756 & 478.1476 & & \end{array}$

Statistical Tests:

Normality Test (Shapiro-Wilk) Passed $\quad(\mathrm{P}=0.9941)$

W Statistic $=0.9877 \quad$ Significance Level $=0.0500$

Constant Variance Test Passed $(\mathrm{P}=0.5567)$

Fit Equation Description:

[Variables]

$\mathrm{x}=\operatorname{col}(4)$

$\mathrm{y}=\operatorname{col}(5)$

reciprocal_y $=1 / \operatorname{abs}(\mathrm{y})$

reciprocal_ysquare $=1 / \mathrm{y}^{\wedge} 2$

reciprocal_pred $=1 / \mathrm{abs}(\mathrm{f})$

reciprocal_predsqr $=1 / \mathrm{f}^{\wedge} 2$

[Parameters]

$\mathrm{a}=\max (\mathrm{y})-\min (\mathrm{y}) "$ Auto $\{$ previous: 51.2683$\}$ \}

$\mathrm{b}=\mathrm{if}(\mathrm{xwtr}(\mathrm{x}, \mathrm{y}-\min (\mathrm{y}), .5) \diamond 0, \mathrm{xwtr}(\mathrm{x}, \mathrm{y}-\min (\mathrm{y}), .5) / 4,1)$ "Auto \{\{previous: -0.283592$\}\}$

$\mathrm{x} 0=\mathrm{x} 50(\mathrm{x}, \mathrm{y}-\min (\mathrm{y}), 5)$ "Auto $\{$ previous: 5.15981$\}$ \}

y0 $=\min (y) "$ Auto $\{\{$ previous: 77.2766$\}\}$

[Equation]

$\mathrm{f}=\mathrm{y} 0+\mathrm{a} /(1+\exp (-(\mathrm{x}-\mathrm{x} 0) / \mathrm{b}))$

fit $f$ to $y$ 


\section{7-IQBA_Mannose_378nm_4}

Nonlinear Regression

Data Source: Data 1 in Mannose Titration

Equation: Sigm oidal, Sigmoid, 4 Parameter

$\mathrm{f}=\mathrm{y} 0+\mathrm{a} /(1+\exp (-(\mathrm{x}-\mathrm{x} 0) / \mathrm{b}))$
Wednesday, September 21, 2011, 12:36:55 PM

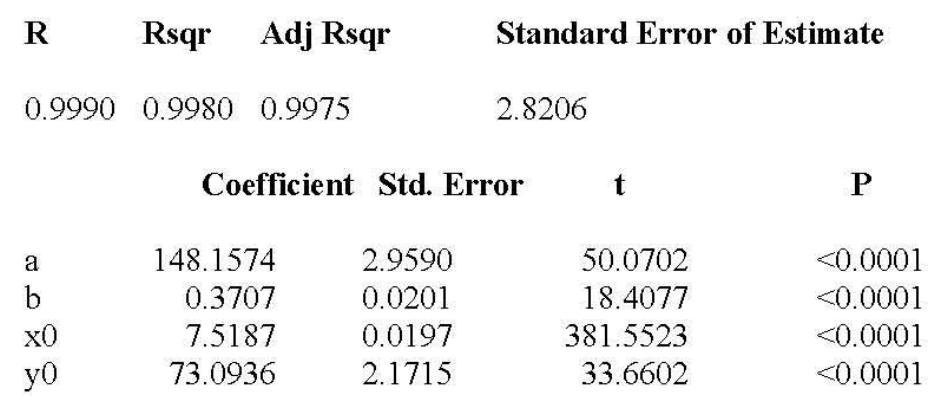

Analysis of Variance:

\begin{tabular}{lrrr} 
& \multicolumn{1}{c}{ DF } & \multicolumn{1}{c}{ SS } & \multicolumn{1}{c}{ MS } \\
Regression 4 & 375152.5518 & 93788.1380 \\
Residual & 12 & 95.4700 & 7.9558 \\
Total & 16 & 375248.0218 & 23453.0014
\end{tabular}

Corrected for the mean of the observations:

\begin{tabular}{lrrrrr} 
& DF & SS & MS & \multicolumn{1}{c}{ F } & P \\
Regression 3 & 47470.7226 & 15823.5742 & 1988.9272 & $<0.0001$ \\
Residual & 12 & 95.4700 & 7.9558 & & \\
Total & 15 & 47566.1926 & 3171.0795 & &
\end{tabular}

\section{Statistical Tests:}

Normality Test (Shapiro-Wilk) Passed $(\mathrm{P}=0.3841)$

W Statistic $=0.9428 \quad$ Significance Level $=0.0500$

Constant Variance Test

Passed $(\mathrm{P}=0.2766)$

Fit Equation Description:

[Variables]

$\mathrm{x}=\operatorname{col}(6)$

$\mathrm{y}=\operatorname{col}(7)$

reciprocal_y $=1 / \mathrm{abs}(\mathrm{y})$

reciprocal_ysquare $=1 / \mathrm{y}^{\wedge} 2$

reciprocal_pred $=1 / \mathrm{abs}(\mathrm{f})$

reciprocal_predsqr $=1 / \mathrm{f}^{\wedge} 2$

[Parameters]

$\mathrm{a}=\max (\mathrm{y})-\min (\mathrm{y}) "$ Auto $\{$ previous: 148.157$\}$ \}

$\mathrm{b}=\mathrm{if}(\mathrm{xwtr}(\mathrm{x}, \mathrm{y}-\min (\mathrm{y}), .5) \diamond 0, \mathrm{xwtr}(\mathrm{x}, \mathrm{y}-\min (\mathrm{y}), .5) / 4,1)$ "Auto \{\{previous: 0.370658$\}\}$

$\mathrm{x} 0=\mathrm{x} 50(\mathrm{x}, \mathrm{y}-\min (\mathrm{y}), 5)$ "Auto $\{\{$ previous: 7.51872$\}\}$

y0 $=\min (y) " A u t o ~\{\{$ previous: 73.0936$\}\}$

[Equation]

$\mathrm{f}=\mathrm{y} 0+\mathrm{a} /(1+\exp (-(\mathrm{x}-\mathrm{x} 0) / \mathrm{b}))$

fit $f$ to $y$ 


\section{7-IQBA_Sorbitol_344nm_1}

Nonlinear Regression

Data Source: Data 2 in Sorbitol Titration

Equation: Sigm oidal, Sigmoid, 4 Parameter

$\mathrm{f}=\mathrm{y} 0+\mathrm{a} /(1+\exp (-(\mathrm{x}-\mathrm{x} 0) / \mathrm{b}))$
Wednesday, September 21, 2011, 1:07:54 PM

\section{R Rsqr Adj Rsqr Standard Error of Estimate \\ $\begin{array}{llll}0.9966 & 0.9932 & 0.9918 & 31.2642\end{array}$}

\section{Coefficient Std. Error}

$\begin{array}{lrrrr}\text { a } & 739.8470 & 18.8404 & 39.2692 & <0.0001 \\ \mathrm{~b} & 0.3590 & 0.0412 & 8.7154 & <0.0001 \\ \text { x0 } & 6.7244 & 0.0494 & 136.0571 & <0.0001 \\ \text { y0 } & 26.7927 & 12.3274 & 2.1734 & 0.0462\end{array}$

Analysis of Variance:

\begin{tabular}{lrrr} 
& DF & \multicolumn{1}{c}{ SS } & \multicolumn{1}{c}{ MS } \\
Regression 4 & 5353478.9203 & 1338369.7301 \\
Residual & 15 & 14661.7901 & 977.4527 \\
Total & 19 & 5368140.7104 & 282533.7216
\end{tabular}

Corrected for the mean of the observations:

\begin{tabular}{lrrrrr} 
& \multicolumn{1}{c}{ DF } & SS & MS & \multicolumn{1}{c}{ F } & P \\
Regression 3 & 2132030.0898 & 710676.6966 & 727.0702 & $<0.0001$ \\
Residual & 15 & 14661.7901 & 977.4527 & & \\
Total & 18 & 2146691.8799 & 119260.6600 & &
\end{tabular}

\section{Statistical Tests:}

Normality Test (Shapiro-Wilk) $\quad$ Failed $\quad(P=0.0313)$

W Statistic $=0.8893 \quad$ Significance Level $=0.0500$

Constant Variance Test Failed $(\mathrm{P}=0.0309)$

Fit Equation Description:

[Variables]

$\mathrm{x}=\operatorname{col}(1)$

$\mathrm{y}=\operatorname{col}(2)$

reciprocal_y $=1 / \mathrm{abs}(\mathrm{y})$

reciprocal_ysquare $=1 / \mathrm{y}^{\wedge} 2$

reciprocal_pred $=1 / \mathrm{abs}(\mathrm{f})$

reciprocal_predsqr $=1 / \mathrm{f}^{\wedge} 2$

[Parameters]

$\mathrm{a}=\max (\mathrm{y})-\min (\mathrm{y}) "$ Auto $\{$ previous: 739.847$\}$ \}

$\mathrm{b}=\mathrm{if}(\mathrm{xwtr}(\mathrm{x}, \mathrm{y}-\min (\mathrm{y}), .5)>0, \mathrm{xwtr}(\mathrm{x}, \mathrm{y}-\min (\mathrm{y}), .5) / 4,1)$ "Auto \{\{previous: 0.358981$\}\}$

$\mathrm{x} 0=\mathrm{x} 50(\mathrm{x}, \mathrm{y}-\min (\mathrm{y}), .5)$ "Auto $\{$ previous: 6.72438$\}$ \}

y0 $=\min (y) " A u t o ~\{\{$ previous: 26.7927$\}\}$

[Equation]

$\mathrm{f}=\mathrm{y} 0+\mathrm{a} /(1+\exp (-(\mathrm{x}-\mathrm{x} 0) / \mathrm{b}))$

fit $\mathrm{f}$ to $\mathrm{y}$ 
7-IQBA_Sorbitol_344nm_2

Nonlinear Regression

Data Source: Data 1 in Sorbitol Titration

Equation: Sigm oidal, Sigmoid, 4 Parameter

$\mathrm{f}=\mathrm{y} 0+\mathrm{a} /(1+\exp (-(\mathrm{x}-\mathrm{x} 0) / \mathrm{b}))$

\section{R Rsqr Adj Rsqr Standard Error of Estimate}

$\begin{array}{lll}0.9993 & 0.9987 & 0.9984\end{array}$

15.1430

Coefficient Std. Error

$\mathbf{t}$

$\begin{array}{lrrrr}\text { a } & 856.1416 & 10.3947 & 82.3635 & <0.0001 \\ \text { b } & 0.4450 & 0.0190 & 23.4376 & <0.0001 \\ \text { x0 } & 6.7965 & 0.0235 & 288.6634 & <0.0001 \\ \text { y0 } & 21.4203 & 6.4392 & 3.3265 & 0.0046\end{array}$

Analysis of Variance:

\begin{tabular}{lrrr} 
& DF & \multicolumn{1}{c}{ SS } & \multicolumn{1}{c}{ MS } \\
Regression 4 & 6421046.5269 & 1605261.6317 \\
Residual & 15 & 3439.6558 & 229.3104 \\
Total & 19 & 6424486.1827 & 338130.8517
\end{tabular}

Corrected for the mean of the observations:

\begin{tabular}{lrrrrr} 
& \multicolumn{1}{c}{ DF } & SS & MS & \multicolumn{1}{c}{ F } & P \\
Regression 3 & 2568757.0373 & 856252.3458 & 3734.0321 & $<0.0001$ \\
Residual & 15 & 3439.6558 & 229.3104 & & \\
Total & 18 & 2572196.6931 & 142899.8163 & &
\end{tabular}

\section{Statistical Tests:}

Normality Test (Shapiro-Wilk) $\quad$ Failed $\quad(\mathrm{P}=0.0009)$

W Statistic $=0.7929 \quad$ Significance Level $=0.0500$

Constant Variance Test Passed $(\mathrm{P}=0.1862)$

\section{Fit Equation Description:}

[Variables]

$\mathrm{x}=\operatorname{col}(1)$

$\mathrm{y}=\operatorname{col}(2)$

reciprocal_y $=1 / \operatorname{abs}(\mathrm{y})$

reciprocal_ysquare $=1 / \mathrm{y}^{\wedge} 2$

reciprocal_pred $=1 / \mathrm{abs}(\mathrm{f})$

reciprocal_predsqr $=1 / \mathrm{f}^{\wedge} 2$

[Parameters]

$\mathrm{a}=\max (\mathrm{y})-\min (\mathrm{y})$ "Auto $\{$ previous: 856.142$\}$ \}

$\mathrm{b}=\mathrm{if}(\mathrm{xwtr}(\mathrm{x}, \mathrm{y}-\min (\mathrm{y}), .5)>0, \mathrm{xwtr}(\mathrm{x}, \mathrm{y}-\min (\mathrm{y}), .5) / 4,1)$ "Auto \{\{previous: 0.445004$\}$ \}

$\mathrm{x} 0=\mathrm{x} 50(\mathrm{x}, \mathrm{y}-\min (\mathrm{y}), .5)$ "Auto $\{\{$ previous: 6.79655$\}\}$

y0 $=\min (y) " A u t o\{\{$ previous: 21.4203$\}\}$

[Equation]

$\mathrm{f}=\mathrm{y} 0+\mathrm{a} /(1+\exp (-(\mathrm{x}-\mathrm{x} 0) / \mathrm{b}))$

fit $\mathrm{f}$ to $\mathrm{y}$ 


\section{7-IQBA_Sorbitol_378nm_1}

Nonlinear Regression

Wednesday, September 21, 2011, 1:08:04 PM

Data Source: Data 2 in Sorbitol Titration

Equation: Sigm oidal, Sigmoid, 4 Parameter

$\mathrm{f}=\mathrm{y} 0+\mathrm{a} /(1+\exp (-(\mathrm{x}-\mathrm{x} 0) / \mathrm{b}))$

$\begin{array}{llll}\text { R } & \text { Rsqr } & \text { Adj Rsqr } & \text { Standard Error of Estimate } \\ 0.8006 & 0.6410 & 0.3717 & 7.2684\end{array}$

Coefficient Std. Error t $\quad$ P

$\begin{array}{lrrrr}\text { a } & 16.7530 & 10.0720 & 1.6633 & 0.1716 \\ \text { b } & -0.3581 & 0.6138 & -0.5833 & 0.5910 \\ \text { x0 } & 4.2745 & 0.7258 & 5.8891 & 0.0042 \\ \text { y0 } & 116.6072 & 6.4332 & 18.1258 & <0.0001\end{array}$

Analysis of Variance:

\begin{tabular}{lcrr} 
& DF & \multicolumn{1}{c}{ SS } & \multicolumn{1}{c}{ MS } \\
Regression & 4 & 126312.2583 & 31578.0646 \\
Residual & 4 & 211.3165 & 52.8291 \\
Total & 8 & 126523.5748 & 15815.4468
\end{tabular}

Corrected for the mean of the observations:

$\begin{array}{lccccc} & \text { DF } & \text { SS } & \text { MS } & \text { F } & \text { P } \\ \text { Regression } & 3 & 377.2664 & 125.7555 & 2.3804 & 0.2105 \\ \text { Residual } & 4 & 211.3165 & 52.8291 & & \\ \text { Total } & 7 & 588.5828 & 84.0833 & & \end{array}$

\section{Statistical Tests:}

Normality Test (Shapiro-Wilk) Passed $\quad(P=0.2072)$

W Statistic $=0.8844 \quad$ Significance Level $=0.0500$

Constant Variance Test $\quad$ Failed $\quad(\mathrm{P}=0.0096)$

Fit Equation Description:

[Variables]

$\mathrm{x}=\operatorname{col}(3)$

$\mathrm{y}=\operatorname{col}(4)$

reciprocal_y $=1 / \mathrm{abs}(\mathrm{y})$

reciprocal ysquare $=1 / \mathrm{y}^{\wedge} 2$

reciprocal pred $=1 / \mathrm{abs}(\mathrm{f})$

reciprocal predsqr $=1 / \mathrm{f}^{\wedge} 2$

[Parameters]

$\mathrm{a}=\max (\mathrm{y})-\min (\mathrm{y}) "$ Auto $\{$ previous: 16.753$\}$ \}

$\mathrm{b}=\mathrm{if}(\mathrm{xwtr}(\mathrm{x}, \mathrm{y}-\min (\mathrm{y}), .5) \circlearrowright 0, \mathrm{xwtr}(\mathrm{x}, \mathrm{y}-\min (\mathrm{y}), .5) / 4,1)$ "Auto \{\{previous: -0.358085$\}\}$

$\mathrm{x} 0=\mathrm{x} 50(\mathrm{x}, \mathrm{y}-\mathrm{min}(\mathrm{y}), 5)$ "Auto \{\{previous: 4.27446$\}\}$

y0 $=\min (y) "$ Auto $\{\{$ previous: 116.607$\}\}$

[Equation]

$\mathrm{f}=\mathrm{y} 0+\mathrm{a} /(1+\exp (-(\mathrm{x}-\mathrm{x} 0) / \mathrm{b}))$

fit $\mathrm{f}$ to $\mathrm{y}$ 
7-IQBA_Sorbitol_378nm_2

Nonlinear Regression

Wednesday, September 21, 2011, 1:08:18 PM

Data Source: Data 2 in Sorbitol Titration

Equation: Sigm oidal, Sigmoid, 4 Parameter

$\mathrm{f}=\mathrm{y} 0+\mathrm{a} /(1+\exp (-(\mathrm{x}-\mathrm{x} 0) / \mathrm{b}))$

$\begin{array}{llll}\text { R } & \text { Rsqr } & \text { Adj Rsqr } & \text { Standard Error of Estimate } \\ 0.9856 & 0.9713 & 0.9627 & 12.0657\end{array}$

Coefficient Std. Error t $\quad$ P

$\begin{array}{lrrrr}\text { a } & 146.5266 & 9.6877 & 15.1250 & <0.0001 \\ \text { b } & 0.2547 & 0.0695 & 3.6646 & 0.0044 \\ \text { x0 } & 6.7336 & 0.0840 & 80.1377 & <0.0001 \\ \text { y0 } & 115.0223 & 8.0590 & 14.2725 & <0.0001\end{array}$

Analysis of Variance:

\begin{tabular}{lrrr} 
& \multicolumn{1}{c}{ DF } & \multicolumn{1}{c}{ SS } & \multicolumn{1}{c}{ MS } \\
Regression 4 & 722378.6590 & 180594.6647 \\
Residual & 10 & 1455.8166 & 145.5817 \\
Total & 14 & 723834.4756 & 51702.4625
\end{tabular}

Corrected for the mean of the observations:

\begin{tabular}{lrrrrc} 
& \multicolumn{1}{c}{ DF } & SS & MS & F & P \\
Regression 3 & 49343.8637 & 16447.9546 & 112.9810 & $<0.0001$ \\
Residual & 10 & 1455.8166 & 145.5817 & & \\
Total & 13 & 50799.6803 & 3907.6677 & &
\end{tabular}

Statistical Tests:

Normality Test (Shapiro-Wilk) $\quad$ Failed $\quad(\mathrm{P}=0.0093)$

W Statistic $=0.8213 \quad$ Significance Level $=0.0500$

Constant Variance Test Passed $(\mathrm{P}=0.6047)$

Fit Equation Description:

[Variables]

$\mathrm{x}=\operatorname{col}(5)$

$\mathrm{y}=\operatorname{col}(6)$

reciprocal $\mathrm{y}=1 / \mathrm{abs}(\mathrm{y})$

reciprocal_ysquare $=1 / \mathrm{y}^{\wedge} 2$

reciprocal_pred $=1 / \mathrm{abs}(\mathrm{f})$

reciprocal_predsqr $=1 / \mathrm{f}^{\wedge} 2$

[Parameters]

$\mathrm{a}=\max (\mathrm{y})-\min (\mathrm{y}) "$ Auto $\{$ previous: 146.527$\}$ \}

$\mathrm{b}=\mathrm{if}(\mathrm{xwtr}(\mathrm{x}, \mathrm{y}-\min (\mathrm{y}), .5)>0, \mathrm{xwtr}(\mathrm{x}, \mathrm{y}-\min (\mathrm{y}), .5) / 4,1)$ "Auto \{\{previous: 0.254708$\}\}$

$\mathrm{x} 0=\mathrm{x} 50(\mathrm{x}, \mathrm{y}-\min (\mathrm{y}), 5)$ "Auto $\{\{$ previous: 6.7336$\}\}$

y0 $=\min (y) " A u t o\{\{$ previous: 115.022$\}$ \}

[Equation]

$\mathrm{f}=\mathrm{y} 0+\mathrm{a} /(1+\exp (-(\mathrm{x}-\mathrm{x} 0) / \mathrm{b}))$

fit $\mathrm{f}$ to $\mathrm{y}$ 
7-IQBA_Sorbitol_378nm_3

Nonlinear Regression

Wednesday, September 21, 2011, 12:51:52 PM

Data Source: Data 1 in Sorbitol Titration

Equation: Sigm oidal, Sigmoid, 4 Parameter

$\mathrm{f}=\mathrm{y} 0+\mathrm{a} /(1+\exp (-(\mathrm{x}-\mathrm{x} 0) / \mathrm{b}))$

$\begin{array}{llll}\text { R } & \text { Rsqr } & \text { Adj Rsqr } & \text { Standard Error of Estimate } \\ 0.9686 & 0.9381 & 0.8762 & 2.5456\end{array}$

Coefficient Std. Error t $\quad$ P

$\begin{array}{lrrrr}\text { a } & 14.0573 & 2.5839 & 5.4403 & 0.0122 \\ \text { b } & -0.1488 & 0.6255 & -0.2378 & 0.8274 \\ \text { x0 } & 3.9698 & 0.2467 & 16.0937 & 0.0005 \\ \text { y0 } & 121.1281 & 1.5539 & 77.9534 & <0.0001\end{array}$

Analysis of Variance:

\begin{tabular}{lrrr} 
& DF & \multicolumn{1}{c}{ SS } & \multicolumn{1}{c}{ MS } \\
Regression & 4 & 114940.5940 & 28735.1485 \\
Residual & 3 & 19.4409 & 6.4803 \\
Total & 7 & 114960.0349 & 16422.8621
\end{tabular}

Corrected for the mean of the observations:

$\begin{array}{llrrrc} & \text { DF } & \text { SS } & \text { MS } & \text { F } & \text { P } \\ \text { Regression } & 3 & 294.5742 & 98.1914 & 15.1523 & 0.0257 \\ \text { Residual } & 3 & 19.4409 & 6.4803 & & \\ \text { Total } & 6 & 314.0151 & 52.3358 & & \end{array}$

Statistical Tests:

Normality Test (Shapiro-Wilk) $\quad$ Passed $\quad(P=0.4049)$

W Statistic $=0.9113 \quad$ Significance Level $=0.0500$

Constant Variance Test Passed $(P=0.3884)$

Fit Equation Description:

[Variables]

$\mathrm{x}=\operatorname{col}(4)$

$\mathrm{y}=\operatorname{col}(5)$

reciprocal_y $=1 / \mathrm{abs}(\mathrm{y})$

reciprocal_ysquare $=1 / \mathrm{y}^{\wedge} 2$

reciprocal_pred $=1 / \mathrm{abs}(\mathrm{f})$

reciprocal_predsqr $=1 / \mathrm{f}^{\wedge} 2$

[Parameters]

$\mathrm{a}=\max (\mathrm{y})-\min (\mathrm{y}) "$ Auto $\{$ previous: 14.0573$\}$ \}

$\mathrm{b}=\mathrm{if}(\mathrm{xwtr}(\mathrm{x}, \mathrm{y}-\min (\mathrm{y}), .5)>0, \mathrm{xwtr}(\mathrm{x}, \mathrm{y}-\min (\mathrm{y}), .5) / 4,1)$ "Auto \{\{previous: -0.148753$\}\}$

$\mathrm{x} 0=\mathrm{x} 50(\mathrm{x}, \mathrm{y}-\min (\mathrm{y}), .5)$ "Auto \{\{previous: 3.9698$\}$ \}

y0 $=\min (y) "$ Auto $\{\{$ previous: 121.128$\}$ \}

[Equation]

$\mathrm{f}=\mathrm{y} 0+\mathrm{a} /(1+\exp (-(\mathrm{x}-\mathrm{x} 0) / \mathrm{b}))$

fit $\mathrm{f}$ to $\mathrm{y}$ 
7-IQBA_Sorbitol_378nm_4

Nonlinear Regression

Wednesday, September 21, 2011, 12:52:03 PM

Data Source: Data 1 in Sorbitol Titration

Equation: Sigm oidal, Sigmoid, 4 Parameter

$\mathrm{f}=\mathrm{y} 0+\mathrm{a} /(1+\exp (-(\mathrm{x}-\mathrm{x} 0) / \mathrm{b}))$

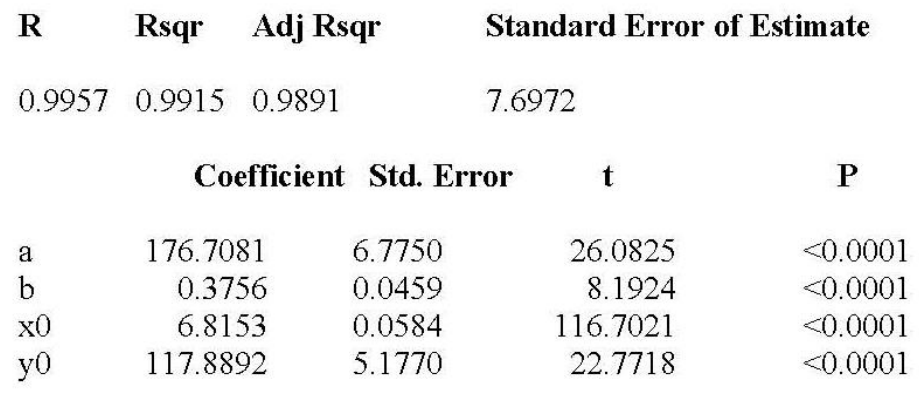

Analysis of Variance:

\begin{tabular}{lrrr} 
& \multicolumn{1}{c}{ DF } & \multicolumn{1}{c}{ SS } & \multicolumn{1}{c}{ MS } \\
Regression 4 & 871478.9364 & 217869.7341 \\
Residual & 11 & 651.7172 & 59.2470 \\
Total & 15 & 872130.6536 & 58142.0436
\end{tabular}

Corrected for the mean of the observations:

$\begin{array}{lrrrrc} & \text { DF } & \text { SS } & \text { MS } & \text { F } & \text { P } \\ \text { Regression } 3 & 75581.4482 & 25193.8161 & 425.2335 & <0.0001 \\ \text { Residual } & 11 & 651.7172 & 59.2470 & & \\ \text { Total } & 14 & 76233.1654 & 5445.2261 & & \end{array}$

Statistical Tests:

Normality Test (Shapiro-Wilk) $\quad$ Passed $\quad(P=0.6029)$

W Statistic $=0.9548 \quad$ Significance Level $=0.0500$

Constant Variance Test $\quad$ Passed $(P=0.4731)$

Fit Equation Description:

[Variables]

$\mathrm{x}=\operatorname{col}(6)$

$\mathrm{y}=\operatorname{col}(7)$

reciprocal $\mathrm{y}=1 / \mathrm{abs}(\mathrm{y})$

reciprocal_ysquare $=1 / \mathrm{y}^{\wedge} 2$

reciprocal pred $=1 / \mathrm{abs}(\mathrm{f})$

reciprocal_predsqr $=1 / \mathrm{f}^{\wedge} 2$

[Parameters]

$\mathrm{a}=\max (\mathrm{y})-\min (\mathrm{y}) "$ Auto $\{$ previous: 176.708$\}$ \}

$\mathrm{b}=\mathrm{if}(\mathrm{xwtr}(\mathrm{x}, \mathrm{y}-\min (\mathrm{y}), .5) \succ 0, \mathrm{xwtr}(\mathrm{x}, \mathrm{y}-\min (\mathrm{y}), .5) / 4,1)$ "Auto \{\{previous: 0.375643$\}\}$

$\mathrm{x} 0=\mathrm{x} 50(\mathrm{x}, \mathrm{y}-\min (\mathrm{y}), .5)$ "Auto $\{\{$ previous: 6.81528$\}$ \}

y0 $=\min (y) "$ Auto $\{\{$ previous: 117.889$\}$ \}

[Equation]

$\mathrm{f}=\mathrm{y} 0+\mathrm{a} /(1+\exp (-(\mathrm{x}-\mathrm{x} 0) / \mathrm{b}))$

fit $\mathrm{f}$ to $\mathrm{y}$ 


\section{7-IQBA_Tagatose_344nm_1}

Nonlinear Regression

Wednesday, September 21, 2011, 12:56:23 PM

Data Source: Data 2 in Tagatose Titration

Equation: Sigm oidal, Sigmoid, 4 Parameter

$\mathrm{f}=\mathrm{y} 0+\mathrm{a} /(1+\exp (-(\mathrm{x}-\mathrm{x} 0) / \mathrm{b}))$

\section{R Rsqr Adj Rsqr Standard Error of Estimate}

$\begin{array}{llll}0.9999 & 0.9997 & 0.9997 & 3.9214\end{array}$

Coefficient Std. Error $\quad t \quad$ P

$\begin{array}{lrrrr}\text { a } & 470.7155 & 2.8014 & 168.0278 & <0.0001 \\ \text { b } & 0.4709 & 0.0106 & 44.5243 & <0.0001 \\ \text { x0 } & 6.8783 & 0.0123 & 557.5450 & <0.0001 \\ \text { y0 } & 17.4612 & 1.6218 & 10.7663 & <0.0001\end{array}$

Analysis of Variance:

\begin{tabular}{lrrr} 
& \multicolumn{1}{c}{ DF } & \multicolumn{1}{c}{ SS } & \multicolumn{1}{c}{ MS } \\
Regression 4 & 1577520.2610 & 394380.0653 \\
Residual & 13 & 199.9071 & 15.3775 \\
Total & 17 & 1577720.1681 & 92807.0687
\end{tabular}

Corrected for the mean of the observations:

$\begin{array}{lrrrrr} & \text { DF } & \text { SS } & \text { MS } & \text { F } & \text { P } \\ \text { Regression } 3 & 710564.7623 & 236854.9208 & 15402.7265 & <0.0001 \\ \text { Residual } & 13 & 199.9071 & 15.3775 & & \\ \text { Total } & 16 & 710764.6694 & 44422.7918 & & \end{array}$

\section{Statistical Tests:}

Normality Test (Shapiro-Wilk) Passed $\quad(P=0.0513)$

W Statistic $=0.8927 \quad$ Significance Level $=0.0500$

Constant Variance Test $\quad$ Passed $(\mathrm{P}=0.4257)$

Fit Equation Description:

[Variables]

$\mathrm{X}=\operatorname{col}(1)$

$\mathrm{y}=\operatorname{col}(2)$

reciprocal $\mathrm{y}=1 / \mathrm{abs}(\mathrm{y})$

reciprocal_ysquare $=1 / \mathrm{y}^{\wedge} 2$

reciprocal_pred $=1 / \mathrm{abs}(\mathrm{f})$

reciprocal_predsqr $=1 / \mathrm{f}^{\wedge} 2$

[Parameters]

$\mathrm{a}=\max (\mathrm{y})-\min (\mathrm{y}) "$ Auto $\{$ previous: 470.715$\}$ \}

$\mathrm{b}=\mathrm{if}(\mathrm{xwtr}(\mathrm{x}, \mathrm{y}-\min (\mathrm{y}), .5) \circlearrowright 0, \mathrm{xwtr}(\mathrm{x}, \mathrm{y}-\min (\mathrm{y}), .5) / 4,1)$ "Auto \{\{previous: 0.470886$\}$ \}

$\mathrm{x} 0=\mathrm{x} 50(\mathrm{x}, \mathrm{y}-\min (\mathrm{y}), 5)$ "Auto $\{$ previous: 6.87826$\}$ \}

y0 $=\min (y) "$ Auto $\{\{$ previous: 17.4612$\}\}$

[Equation]

$\mathrm{f}=\mathrm{y} 0+\mathrm{a} /(1+\exp (-(\mathrm{x}-\mathrm{x} 0) / \mathrm{b}))$

fit $\mathrm{f}$ to $\mathrm{y}$ 
7-IQBA_Tagatose_344nm_2

Nonlinear Regression

Wednesday, September 21, 2011, 1:00:28 PM

Data Source: Data 1 in Tagatose Titration Equation: Sigm oidal, Sigmoid, 4 Parameter $\mathrm{f}=\mathrm{y} 0+\mathrm{a} /(1+\exp (-(\mathrm{x}-\mathrm{x} 0) / \mathrm{b}))$

\begin{tabular}{|c|c|c|c|c|c|}
\hline $\mathbf{R}$ & Rsqr & Adj Rs & & ndard Err & timate \\
\hline \multirow[t]{2}{*}{0.9998} & 0.9996 & 0.9995 & & 748 & \\
\hline & \multicolumn{2}{|c|}{ Coefficient } & Std. Error & $\mathbf{t}$ & $\mathbf{P}$ \\
\hline a & \multicolumn{2}{|c|}{514.9725} & 3.3805 & 152.3361 & $<0.0001$ \\
\hline b & \multicolumn{2}{|c|}{0.4335} & 0.0115 & 37.6488 & $<0.0001$ \\
\hline $\mathrm{x} 0$ & \multicolumn{2}{|c|}{6.8608} & 0.0137 & 501.2176 & $<0.0001$ \\
\hline y0 & \multicolumn{2}{|c|}{20.0916} & 1.9366 & 10.3747 & $<0.0001$ \\
\hline
\end{tabular}

Analysis of Variance:

\begin{tabular}{lrrr} 
& \multicolumn{1}{c}{ DF } & \multicolumn{1}{c}{ SS } & \multicolumn{1}{c}{ MS } \\
Regression 4 & 1920572.9752 & 480143.2438 \\
Residual & 14 & 360.5554 & 25.7540 \\
Total & 18 & 1920933.5306 & 106718.5295
\end{tabular}

Corrected for the mean of the observations:

$\begin{array}{lrrrrc} & \text { DF } & \text { SS } & \text { MS } & \text { F } & \text { P } \\ \text { Regression } 3 & 920696.8033 & 306898.9344 & 11916.5745 & <0.0001 \\ \text { Residual } & 14 & 360.5554 & 25.7540 & & \\ \text { Total } & 17 & 921057.3587 & 54179.8446 & & \end{array}$

\section{Statistical Tests:}

Normality Test (Shapiro-Wilk) Passed $(\mathrm{P}=0.9857)$

W Statistic $=0.9848 \quad$ Significance Level $=0.0500$

Constant Variance Test Passed $(P=0.4869)$

Fit Equation Description:

[Variables]

$\mathrm{x}=\operatorname{col}(1)$

$\mathrm{y}=\operatorname{col}(2)$

reciprocal_y $=1 / \mathrm{abs}(\mathrm{y})$

reciprocal_ysquare $=1 / \mathrm{y}^{\wedge} 2$

reciprocal_pred $=1 / \mathrm{abs}(\mathrm{f})$

reciprocal_predsqr $=1 / \mathrm{f}^{\wedge} 2$

[Parameters]

$\mathrm{a}=\max (\mathrm{y})-\min (\mathrm{y}) "$ Auto $\{\{$ previous: 514.972$\}\}$

$\mathrm{b}=\mathrm{if}(\mathrm{xwtr}(\mathrm{x}, \mathrm{y}-\min (\mathrm{y}), .5)>0, \mathrm{xwtr}(\mathrm{x}, \mathrm{y}-\min (\mathrm{y}), .5) / 4,1)$ "Auto \{\{previous: 0.433482$\}\}$

$\mathrm{x} 0=\mathrm{x} 50(\mathrm{x}, \mathrm{y}-\min (\mathrm{y}), 5)$ "Auto \{\{previous: 6.86083$\}$ \}

y0 $=\min (y) "$ Auto $\{\{$ previous: 20.0916$\}\}$

[Equation]

$\mathrm{f}=\mathrm{y} 0+\mathrm{a} /(1+\exp (-(\mathrm{x}-\mathrm{x} 0) / \mathrm{b}))$

fit $f$ to $y$ 


\section{7-IQBA_Tagatose_378nm_1}

Nonlinear Regression

Wednesday, September 21, 2011, 12:56:30 PM

Data Source: Data 2 in Tagatose Titration

Equation: Sigm oidal, Sigmoid, 4 Parameter

$\mathrm{f}=\mathrm{y} 0+\mathrm{a} /(1+\exp (-(\mathrm{x}-\mathrm{x} 0) / \mathrm{b}))$

$\begin{array}{llll}\text { R } & \text { Rsqr } & \text { Adj Rsqr } & \text { Standard Error of Estimate } \\ 0.9974 & 0.9949 & 0.9937 & 6.0477\end{array}$

Coefficient Std. Error t $\quad$ P

$\begin{array}{lrrrr}\text { a } & 184.7042 & 5.2863 & 34.9403 & <0.0001 \\ \text { b } & 0.5932 & 0.0547 & 10.8462 & <0.0001 \\ \text { x0 } & 5.2163 & 0.0594 & 87.7818 & <0.0001 \\ \text { y0 } & 106.1662 & 4.2286 & 25.1068 & <0.0001\end{array}$

Analysis of Variance:

\begin{tabular}{lrrr} 
& \multicolumn{1}{c}{ DF } & \multicolumn{1}{c}{ SS } & \multicolumn{1}{c}{ MS } \\
Regression 4 & 952409.8870 & 238102.4718 \\
Residual & 13 & 475.4765 & 36.5751 \\
Total & 17 & 952885.3635 & 56052.0802
\end{tabular}

Corrected for the mean of the observations:

\begin{tabular}{lrrrrc} 
& \multicolumn{1}{c}{ DF } & SS & MS & F & P \\
Regression 3 & 92201.0867 & 30733.6956 & 840.2898 & $<0.0001$ \\
Residual & 13 & 475.4765 & 36.5751 & & \\
Total & 16 & 92676.5632 & 5792.2852 & &
\end{tabular}

Statistical Tests:

Normality Test (Shapiro-Wilk) $\quad$ Passed $\quad(P=0.1419)$

W Statistic $=0.9190 \quad$ Significance Level $=0.0500$

Constant Variance Test Failed $(\mathrm{P}=0.0091)$

Fit Equation Description:

[Variables]

$\mathrm{x}=\operatorname{col}(1)$

$\mathrm{y}=\operatorname{col}(3)$

reciprocal_y $=1 / \mathrm{abs}(\mathrm{y})$

reciprocal_ysquare $=1 / \mathrm{y}^{\wedge} 2$

reciprocal_pred $=1 / \mathrm{abs}(\mathrm{f})$

reciprocal_predsqr $=1 / \mathrm{f}^{\wedge} 2$

[Parameters]

$\mathrm{a}=\max (\mathrm{y})-\min (\mathrm{y}) "$ Auto $\{\{$ previous: 184.704$\}$ \}

$\mathrm{b}=\mathrm{if}(\mathrm{xwtr}(\mathrm{x}, \mathrm{y}-\min (\mathrm{y}), .5) \diamond 0, \mathrm{xwtr}(\mathrm{x}, \mathrm{y}-\min (\mathrm{y}), .5) / 4,1)$ "Auto \{\{previous: 0.593175$\}\}$

$\mathrm{x} 0=\mathrm{x} 50(\mathrm{x}, \mathrm{y}-\mathrm{min}(\mathrm{y}), 5)$ "Auto $\{$ previous: 5.21632$\}\}$

y0 $=\min (y) " A u t o\{\{$ previous: 106.166$\}$ \}

[Equation]

$\mathrm{f}=\mathrm{y} 0+\mathrm{a} /(1+\exp (-(\mathrm{x}-\mathrm{x} 0) / \mathrm{b}))$

fit $\mathrm{f}$ to $\mathrm{y}$ 
7-IQBA_Tagatose_378nm_2

Nonlinear Regression

Data Source: Data 1 in Tagatose Titration

Equation: Sigm oidal, Sigmoid, 4 Parameter

$\mathrm{f}=\mathrm{y} 0+\mathrm{a} /(1+\exp (-(\mathrm{x}-\mathrm{x} 0) / \mathrm{b}))$
Wednesday, September 21, 2011, 1:00:35 PM

\begin{tabular}{|c|c|c|c|c|c|}
\hline $\mathbf{R}$ & Rsqr & \multicolumn{2}{|c|}{ Adj Rsqr } & \multicolumn{2}{|c|}{ Standard Error of Estimate } \\
\hline \multirow[t]{2}{*}{0.9969} & 0.9938 & 0.9925 & & 77 & \\
\hline & $\mathrm{Cot}$ & efficient & Std. Error & $\mathbf{t}$ & $\mathbf{P}$ \\
\hline $\mathrm{a}$ & 206.12 & & 5.5507 & 37.1341 & $<0.0001$ \\
\hline $\mathrm{b}$ & 0.50 & & 0.0494 & 10.1617 & $<0.0001$ \\
\hline x0 & 5.07 & & 0.0499 & 101.7657 & $<0.0001$ \\
\hline y0 & 109.90 & & 4.4670 & 24.6039 & $<0.0001$ \\
\hline
\end{tabular}

Analysis of Variance:

\begin{tabular}{lrrr} 
& \multicolumn{1}{c}{ DF } & \multicolumn{1}{c}{ SS } & \multicolumn{1}{c}{ MS } \\
Regression 4 & 1179972.8110 & 294993.2028 \\
Residual & 14 & 772.3826 & 55.1702 \\
Total & 18 & 1180745.1936 & 65596.9552
\end{tabular}

Corrected for the mean of the observations:

$\begin{array}{lrrrrr} & \text { DF } & \text { SS } & \text { MS } & \text { F } & \text { P } \\ \text { Regression 3 } & 124399.7924 & 41466.5975 & 751.6124 & <0.0001 \\ \text { Residual } 14 & 772.3826 & 55.1702 & & \\ \text { Total } & 17 & 125172.1751 & 7363.0691 & & \end{array}$

\section{Statistical Tests:}

Normality Test (Shapiro-Wilk) Passed $\quad(P=0.2259)$

W Statistic $=0.9337 \quad$ Significance Level $=0.0500$

Constant Variance Test Passed $(\mathrm{P}=0.1693)$

Fit Equation Description:

[Variables]

$\mathrm{x}=\operatorname{col}(1)$

$\mathrm{y}=\operatorname{col}(3)$

reciprocal_y $=1 / \operatorname{abs}(\mathrm{y})$

reciprocal_ysquare $=1 / \mathrm{y}^{\wedge} 2$

reciprocal pred $=1 / \mathrm{abs}(\mathrm{f})$

reciprocal predsqr $=1 / \mathrm{f}^{\wedge} 2$

[Parameters]

$\mathrm{a}=\max (\mathrm{y})-\min (\mathrm{y}) "$ Auto $\{$ previous: 206.121$\}$ \}

$\mathrm{b}=\mathrm{if}(\mathrm{xwtr}(\mathrm{x}, \mathrm{y}-\min (\mathrm{y}), .5)>0, \mathrm{xwtr}(\mathrm{x}, \mathrm{y}-\min (\mathrm{y}), .5) / 4,1)$ "Auto \{\{previous: 0.501877$\}\}$

$\mathrm{x} 0=\mathrm{x} 50(\mathrm{x}, \mathrm{y}-\mathrm{min}(\mathrm{y}), 5)$ "Auto $\{\{$ previous: 5.07922$\}\}$

y0 $=\min (\mathrm{y}) "$ Auto $\{$ previous: 109.906$\}\}$

[Equation]

$\mathrm{f}=\mathrm{y} 0+\mathrm{a} /(1+\exp (-(\mathrm{x}-\mathrm{x} 0) / \mathrm{b}))$

fit $f$ to $y$ 


\section{4-POBA_430nm_1}

Nonlinear Regression

Wednesday, September 21, 2011, 2:43:00 PM

Data Source: Data 1 in Titration Reports

Equation: Sigm oidal, Sigmoid, 4 Parameter

$\mathrm{f}=\mathrm{y} 0+\mathrm{a} /(1+\exp (-(\mathrm{x}-\mathrm{x} 0) / \mathrm{b}))$

R Rsqr Adj Rsqr Standard Error of Estimate

$\begin{array}{llll}0.9995 & 0.9989 & 0.9987 & 3.6786\end{array}$

Coefficient Std. Error $\quad t \quad$ P

$\begin{array}{lrrrr}\text { a } & 241.1165 & 3.2042 & 75.2513 & <0.0001 \\ \text { b } & -0.4891 & 0.0219 & -22.3752 & <0.0001 \\ \text { x0 } & 7.9988 & 0.0243 & 329.8446 & <0.0001 \\ \text { y0 } & 7.8607 & 2.5946 & 3.0297 & 0.0105\end{array}$

Analysis of Variance:

\begin{tabular}{lrrr} 
& \multicolumn{1}{c}{ DF } & SS & \multicolumn{1}{c}{ MS } \\
Regression 4 & 588552.6652 & 147138.1663 \\
Residual & 12 & 162.3838 & 13.5320 \\
Total & 16 & 588715.0491 & 36794.6906
\end{tabular}

Corrected for the mean of the observations:

\begin{tabular}{lrrrrr} 
& \multicolumn{1}{c}{ DF } & SS & MS & F & P \\
Regression 3 & 150667.7267 & 50222.5756 & 3711.3977 & $<0.0001$ \\
Residual & 12 & 162.3838 & 13.5320 & & \\
Total & 15 & 150830.1105 & 10055.3407 & &
\end{tabular}

\section{Statistical Tests:}

Normality Test (Shapiro-Wilk) Passed $\quad(P=0.6650)$

W Statistic $=0.9602 \quad$ Significance Level $=0.0500$

Constant Variance Test Failed $(\mathrm{P}=0.0120)$

Fit Equation Description:

[Variables]

$\mathrm{x}=\operatorname{col}(11)$

$\mathrm{y}=\operatorname{col}(12)$

reciprocal_y $=1 / \mathrm{abs}(\mathrm{y})$

reciprocal ysquare $=1 / \mathrm{y}^{\wedge} 2$

reciprocal_pred $=1 / \mathrm{abs}(\mathrm{f})$

reciprocal_predsqr $=1 / \mathrm{f}^{\wedge} 2$

[Parameters]

$\mathrm{a}=\max (\mathrm{y})-\min (\mathrm{y}) "$ Auto $\{$ previous: 241.116$\}$ \}

$\mathrm{b}=\mathrm{if}(\mathrm{xwtr}(\mathrm{x}, \mathrm{y}-\min (\mathrm{y}), .5) \diamond 0, \mathrm{xwtr}(\mathrm{x}, \mathrm{y}-\min (\mathrm{y}), .5) / 4,1)$ "Auto \{\{previous: -0.489059$\}\}$

$\mathrm{x} 0=\mathrm{x} 50(\mathrm{x}, \mathrm{y}-\min (\mathrm{y}), 5)$ "Auto \{\{previous: 7.99879$\}$ \}

y0 $=\min (y) "$ Auto $\{\{$ previous: 7.86071$\}$ \}

[Equation]

$\mathrm{f}=\mathrm{y} 0+\mathrm{a} /(1+\exp (-(\mathrm{x}-\mathrm{x} 0) / \mathrm{b}))$

fit $\mathrm{f}$ to $\mathrm{y}$ 


\section{4-POBA_430nm_2}

\begin{tabular}{|c|c|c|c|c|c|c|}
\hline \multicolumn{7}{|c|}{ Nonlinear Regression } \\
\hline \multicolumn{7}{|c|}{$\begin{array}{l}\text { Data Source: Data } 1 \text { in All for POBA.JNB } \\
\text { Equation: Standard Curves, Four Parameter Logistic Curve } \\
\mathrm{fl}=\min +(\max -m i n) /\left(1+\mathrm{abs}(\mathrm{x} / \mathrm{EC} 50)^{\wedge} \text { Hillslope }\right) \\
\mathrm{f} 2=\min +(\max -\mathrm{min})^{*}\left(\mathrm{abs}(\mathrm{x} / \mathrm{EC} 50)^{\wedge} \mathrm{abs}(\text { Hillslope })\right) /\left(1+\left(\mathrm{abs}(\mathrm{x} / \mathrm{EC} 50)^{\wedge} \text { abs }(\text { Hillslope })\right)\right) \\
\mathrm{f}=\mathrm{if}(\mathrm{x}<=0, \text { if }(\text { Hillslope }>0, \max , \mathrm{min}), \text { if }(\text { Hillslope }>0, \mathrm{f} 1, \mathrm{f} 2))\end{array}$} \\
\hline $\mathbf{R}$ & Rsqr & Adj Rsc & Sta & adard Erro & timate & \\
\hline \multirow[t]{2}{*}{0.9994} & 0.9987 & 0.9985 & 4.2 & & & \\
\hline & $\mathrm{Co}$ & efficient & Std. Error & $\mathbf{t}$ & $\mathbf{P}$ & VIF \\
\hline $\min$ & 7.57 & & 2.0164 & 3.7583 & 0.0019 & 1.6971 \\
\hline $\max$ & 247.66 & & 1.5980 & 154.9899 & $<0.0001$ & 1.3658 \\
\hline EC50 & 7.99 & & 0.0249 & 320.9172 & $<0.0001$ & 1.4909 \\
\hline Hillslope & e 16.61 & & 0.7875 & 21.0942 & $<0.0001$ & 1.5182 \\
\hline
\end{tabular}

\section{Analysis of Variance:}

Uncorrected for the mean of the observations:

\begin{tabular}{lrrr} 
& DF & \multicolumn{1}{c}{ SS } & \multicolumn{1}{c}{ MS } \\
Regression 4 & 588732.2475 & 147183.0619 \\
Residual & 15 & 267.2276 & 17.8152 \\
Total & 19 & 588999.4752 & 30999.9724
\end{tabular}

Corrected for the mean of the observations:

\begin{tabular}{lrrrrr} 
& \multicolumn{1}{c}{ DF } & SS & MS & F & P \\
Regression 3 & 211818.4923 & 70606.1641 & 3963.2596 & $<0.0001$ \\
Residual & 15 & 267.2276 & 17.8152 & & \\
Total & 18 & 212085.7200 & 11782.5400 & &
\end{tabular}

\section{Statistical Tests:}

PRESS $\quad 410.6307$

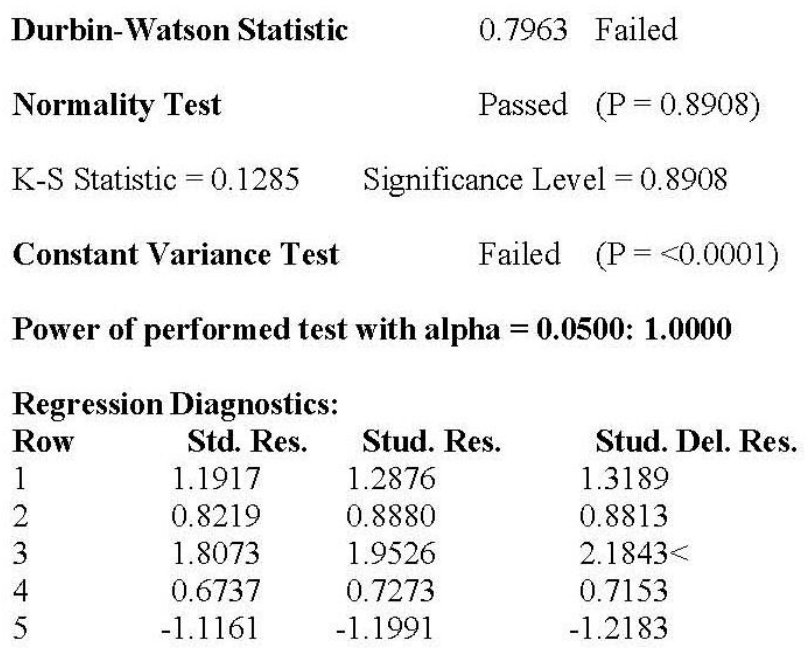




\section{4-POBA_Fructose_430nm_1}

Nonlinear Regression

Wednesday, September 21, 2011, 2:45:05 PM

Data Source: Data 1 in Titration Reports

Equation: Sigm oidal, Sigmoid, 4 Parameter

$\mathrm{f}=\mathrm{y} 0+\mathrm{a} /(1+\exp (-(\mathrm{x}-\mathrm{x} 0) / \mathrm{b}))$

$\begin{array}{llll}\text { R } & \text { Rsqr } & \text { Adj Rsqr } & \text { Standard Error of Estimate } \\ 0.9999 & 0.9997 & 0.9996 & 2.0953\end{array}$

\begin{tabular}{|c|c|c|c|c|}
\hline & Coefficien & Std. Error & $\mathbf{t}$ & $\mathbf{P}$ \\
\hline $\mathrm{a}$ & 236.5612 & 1.8135 & 130.4412 & $<0.0001$ \\
\hline b & -0.4697 & 0.0131 & -35.8096 & $<0.0001$ \\
\hline xo & 6.2555 & 0.0179 & 349.7777 & $<0.0001$ \\
\hline y0 & 10.6522 & 1.0637 & 10.0144 & $<0.0001$ \\
\hline
\end{tabular}

Analysis of Variance:

\begin{tabular}{lrrr} 
& DF & \multicolumn{1}{c}{ SS } & \multicolumn{1}{c}{ MS } \\
Regression & 4 & 221923.3437 & 55480.8359 \\
Residual & 7 & 30.7319 & 4.3903 \\
Total & 11 & 221954.0757 & 20177.6432
\end{tabular}

Corrected for the mean of the observations:

\begin{tabular}{lrrrrr} 
& DF & SS & MS & \multicolumn{1}{c}{ F } & P \\
Regression & 3 & 112010.3265 & 37336.7755 & 8504.4234 & $<0.0001$ \\
Residual & 7 & 30.7319 & 4.3903 & & \\
Total & 10 & 112041.0585 & 11204.1058 & &
\end{tabular}

\section{Statistical Tests:}

Normality Test (Shapiro-Wilk) Passed $(P=0.1772)$

W Statistic $=0.8985 \quad$ Significance Level $=0.0500$

Constant Variance Test Passed $(P=0.0653)$

Fit Equation Description:

[Variables]

$\mathrm{x}=\operatorname{col}(1)$

$\mathrm{y}=\operatorname{col}(2)$

reciprocal y $=1 / \mathrm{abs}(\mathrm{y})$

reciprocal ysquare $=1 / \mathrm{y}^{\wedge} 2$

reciprocal_pred $=1 / \mathrm{abs}(\mathrm{f})$

reciprocal $\_$predsqr $=1 / \mathrm{f}^{\wedge} 2$

[Parameters]

$\mathrm{a}=\max (\mathrm{y})-\min (\mathrm{y}) "$ Auto $\{$ previous: 236.561$\}$ \}

$\mathrm{b}=\mathrm{if}(\mathrm{xwtr}(\mathrm{x}, \mathrm{y}-\min (\mathrm{y}), .5) \diamond 0, \mathrm{xwtr}(\mathrm{x}, \mathrm{y}-\min (\mathrm{y}), .5) / 4,1)$ "Auto \{\{previous: -0.469716$\}\}$

$\mathrm{x} 0=\mathrm{x} 50(\mathrm{x}, \mathrm{y}-\min (\mathrm{y}), .5)$ "Auto $\{\{$ previous: 6.25551$\}\}$

y0 $=\min (y) " A u t o\{\{$ previous: 10.6522$\}\}$

[Equation]

$\mathrm{f}=\mathrm{y} 0+\mathrm{a} /(1+\exp (-(\mathrm{x}-\mathrm{x} 0) / \mathrm{b}))$

fit $f$ to $y$ 


\section{4-POBA_Fructose_430nm_2}

\section{Nonlinear Regression}

\section{Data Source: Data 1 in All for POBA.JNB}

Equation: Standard Curves, Four Parameter Logistic Curve

$\mathrm{fl}=\min +(\max -\min ) /\left(1+\operatorname{abs}(\mathrm{x} / \mathrm{EC} 50)^{\wedge}\right.$ Hillslope $)$

$\mathrm{f} 2=\min +(\max -\min )^{*}\left(\operatorname{abs}(\mathrm{x} / \mathrm{EC} 50)^{\wedge} \mathrm{abs}(\right.$ Hillslope $\left.)\right) /\left(1+\left(\operatorname{abs}(\mathrm{x} / \mathrm{EC} 50)^{\wedge} \mathrm{abs}(\right.\right.$ Hillslope $\left.\left.)\right)\right)$

$\mathrm{f}=\mathrm{if}(\mathrm{x}<=0$, if(Hillslope $>0, \max , \mathrm{min})$, if(Hillslope $>0, \mathrm{f} 1, \mathrm{f} 2)$ )

\section{R Rsqr Adj Rsqr Standard Error of Estimate \\ $\begin{array}{llll}0.9996 & 0.9993 & 0.9991 & 3.2307\end{array}$}

\begin{tabular}{|c|c|c|c|c|c|}
\hline & Coefficient & Std. Error & $\mathbf{t}$ & $\mathbf{P}$ & VIF \\
\hline $\min$ & 9.1445 & 1.2640 & 7.2345 & $<0.0001$ & 1.4421 \\
\hline $\max$ & 247.4246 & 1.3511 & 183.1299 & $<0.0001$ & 1.3511 \\
\hline EC50 & 6.2539 & 0.0188 & 331.9677 & $<0.0001$ & 1.4132 \\
\hline Hillslope & 13.5616 & 0.4804 & 28.2314 & $<0.0001$ & 1.3648 \\
\hline
\end{tabular}

Analysis of Variance:

Uncorrected for the mean of the observations:

\begin{tabular}{lrrr} 
& DF & \multicolumn{1}{c}{ SS } & \multicolumn{1}{c}{ MS } \\
Regression 4 & 477917.2403 & 119479.3101 \\
Residual & 15 & 156.5604 & 10.4374 \\
Total & 19 & 478073.8007 & 25161.7790
\end{tabular}

Corrected for the mean of the observations:

\begin{tabular}{lrrrrc} 
& \multicolumn{1}{c}{ DF } & SS & MS & F & P \\
Regression 3 & 214911.8416 & 71637.2805 & 6863.5442 & $<0.0001$ \\
Residual & 15 & 156.5604 & 10.4374 & & \\
Total & 18 & 215068.4020 & 11948.2446 & &
\end{tabular}

\section{Statistical Tests:}

\section{PRESS $\quad 246.6774$}

Durbin-Watson Statistic

1.8329 Passed

Normality Test

$$
\text { Passed }(\mathrm{P}=0.6535)
$$

K-S Statistic $=0.1631 \quad$ Significance Level $=0.6535$

Constant Variance Test $\quad$ Failed $(\mathrm{P}=0.0050)$

Power of performed test with alpha $=0.0500: 1.0000$

Regression Diagnostics:

Row Std. Res. Stud. Res. Stud. Del. Res.

$\begin{array}{llll}1 & 0.5626 & 0.6193 & 0.6061\end{array}$

$2 \quad-0.2084 \quad-0.2294 \quad-0.2220$

$3 \quad 2.0125 \quad 2.2148<\quad 2.6083<$

$\begin{array}{lrrr}4 & 0.7286 & 0.7999 & 0.7898\end{array}$

$5 \quad-1.3320 \quad-1.4505 \quad-1.5113$ 
Data Source: Data 1 in Titration Reports Equation: Sigm oidal, Sigmoid, 4 Parameter $\mathrm{f}=\mathrm{y} 0+\mathrm{a} /(1+\exp (-(\mathrm{x}-\mathrm{x} 0) / \mathrm{b}))$

\section{R Rsqr Adj Rsqr Standard Error of Estimate \\ $\begin{array}{llll}0.9997 & 0.9995 & 0.9993 & 2.6249\end{array}$}

\section{Coefficient Std. Error $\quad t \quad$ P}

$\begin{array}{lrrrr}\text { a } & 222.0919 & 1.7381 & 127.7751 & <0.0001 \\ \text { b } & -0.4572 & 0.0148 & -30.9273 & <0.0001 \\ \text { x0 } & 6.3011 & 0.0167 & 377.1864 & <0.0001 \\ \text { y0 } & 9.6617 & 1.1751 & 8.2217 & <0.0001\end{array}$

Analysis of Variance:

\begin{tabular}{lrrr} 
& \multicolumn{1}{c}{ DF } & \multicolumn{1}{c}{ SS } & \multicolumn{1}{c}{ MS } \\
Regression 4 & 421181.3785 & 105295.3446 \\
Residual & 13 & 89.5721 & 6.8902 \\
Total & 17 & 421270.9506 & 24780.6442
\end{tabular}

Corrected for the mean of the observations:

$\begin{array}{lrrrrc} & \text { DF } & \text { SS } & \text { MS } & \text { F } & \text { P } \\ \text { Regression } 3 & 164111.8557 & 54703.9519 & 7939.4267 & <0.0001 \\ \text { Residual } 13 & 89.5721 & 6.8902 & & \\ \text { Total } & 16 & 164201.4278 & 10262.5892 & & \end{array}$

Statistical Tests:

Normality Test (Shapiro-Wilk) $\quad$ Failed $\quad(\mathrm{P}=<0.0001)$

W Statistic $=0.6309 \quad$ Significance Level $=0.0500$

Constant Variance Test $\quad$ Failed $(\mathrm{P}=0.0051)$

Fit Equation Description:

[Variables]

$\mathrm{x}=\operatorname{col}(3)$

$\mathrm{y}=\operatorname{col}(4)$

reciprocal_y $=1 / \mathrm{abs}(\mathrm{y})$

reciprocal ysquare $=1 / \mathrm{y}^{\wedge} 2$

reciprocal pred $=1 / \mathrm{abs}(\mathrm{f})$

reciprocal_predsqr $=1 / \mathrm{f}^{\wedge} 2$

[Parameters]

$\mathrm{a}=\max (\mathrm{y})-\min (\mathrm{y}) "$ Auto $\{\{$ previous: 222.092$\}$ \}

$\mathrm{b}=$ if $(\mathrm{xwtr}(\mathrm{x}, \mathrm{y}-\min (\mathrm{y}), .5)>0, \mathrm{xwtr}(\mathrm{x}, \mathrm{y}-\min (\mathrm{y}), .5) / 4,1)$ "Auto \{\{previous: -0.457246$\}\}$

$\mathrm{x} 0=\mathrm{x} 50(\mathrm{x}, \mathrm{y}-\min (\mathrm{y}), .5)$ "Auto $\{\{$ previous: 6.30113$\}\}$

y0 $=\min (y)$ "Auto $\{\{$ previous: 9.66166$\}\}$

[Equation]

$\mathrm{f}=\mathrm{y} 0+\mathrm{a} /(1+\exp (-(\mathrm{x}-\mathrm{x} 0) / \mathrm{b}))$

fit $\mathrm{f}$ to $\mathrm{y}$ 


\section{Nonlinear Regression}

Data Source: Data 1 in All for POBA.JNB

Equation: Standard Curves, Four Parameter Logistic Curve

$\mathrm{fl}=\min +(\max -\min ) /\left(1+\operatorname{abs}(\mathrm{x} / \mathrm{EC} 50)^{\wedge}\right.$ Hillslope $)$

$\mathrm{f} 2=\min +(\max -\mathrm{min}) *\left(\operatorname{abs}(\mathrm{x} / \mathrm{EC} 50)^{\wedge} \mathrm{abs}(\right.$ Hillslope $\left.)\right) /\left(1+\left(\operatorname{abs}(\mathrm{x} / \mathrm{EC} 50)^{\wedge} \mathrm{abs}(\right.\right.$ Hillslope $\left.\left.)\right)\right)$

$\mathrm{f}=\mathrm{if}(\mathrm{x}<=0$, if $($ Hillslope $>0, \max , \mathrm{min})$, if(Hillslope $>0, \mathrm{f} 1, \mathrm{f} 2)$ )

\begin{tabular}{|c|c|c|c|c|c|c|}
\hline $\mathbf{R}$ & Rsqr & Adj Rsc & $\mathbf{q r}$ & Idard Erro & imate & \\
\hline 0.9996 & 0.9993 & 0.9992 & & & & \\
\hline & Co & efficient & Std. Error & $\mathbf{t}$ & $\mathbf{P}$ & VIF \\
\hline $\min$ & 8.60 & & 1.1750 & 7.3233 & $<0.0001$ & 1.4674 \\
\hline $\max$ & 230.53 & & 1.2364 & 186.4484 & $<0.0001$ & 1.3690 \\
\hline EC50 & 6.29 & & 0.0183 & 343.3000 & $<0.0001$ & 1.4097 \\
\hline Hillslope & e 13.86 & & 0.4935 & 28.0865 & $<0.0001$ & 1.3965 \\
\hline
\end{tabular}

Analysis of Variance:

Uncorrected for the mean of the observations:

\begin{tabular}{lrrr} 
& \multicolumn{1}{c}{ DF } & SS & \multicolumn{1}{c}{ MS } \\
Regression & 4 & 421314.0008 & 105328.5002 \\
Residual & 15 & 131.4218 & 8.7615 \\
Total & 19 & 421445.4226 & 22181.3380
\end{tabular}

Corrected for the mean of the observations:

$\begin{array}{lrrrrr} & \text { DF } & \text { SS } & \text { MS } & \text { F } & \text { P } \\ \text { Regression 3 } & 187179.0354 & 62393.0118 & 7121.3089 & <0.0001 \\ \text { Residual } & 15 & 131.4218 & 8.7615 & & \\ \text { Total } & 18 & 187310.4572 & 10406.1365 & & \end{array}$

\section{Statistical Tests:}

PRESS $\quad 195.8019$

$\begin{array}{lll}\text { Durbin-Watson Statistic } & 1.6542 & \text { Passed } \\ \text { Normality Test } & \text { Passed } \quad(\mathrm{P}=0.6837)\end{array}$

K-S Statistic $=0.1591 \quad$ Significance Level $=0.6837$

Constant Variance Test $\quad$ Failed $\quad(\mathrm{P}=0.0053)$

Power of performed test with alpha $=0.0500: 1.0000$

Regression Diagnostics:

$\begin{array}{llll}\text { Row } & \text { Std. Res. } & \text { Stud. Res. } & \text { Stud. Del. Res. } \\ 1 & 0.7843 & 0.8632 & 0.8555 \\ 2 & 1.0015 & 1.1022 & 1.1107 \\ 3 & 1.0480 & 1.1529 & 1.1667 \\ 4 & 0.8603 & 0.9440 & 0.9403 \\ 5 & -2.9266< & -3.1877< & -5.4220<\end{array}$


4-POBA_Mannose_430nm_1

Nonlinear Regression

Wednesday, September 21, 2011, 2:50:15 PM

Data Source: Data 1 in Titration Reports

Equation: Sigm oidal, Sigmoid, 4 Parameter

$\mathrm{f}=\mathrm{y} 0+\mathrm{a} /(1+\exp (-(\mathrm{x}-\mathrm{x} 0) / \mathrm{b}))$

$\begin{array}{llll}\text { R } & \text { Rsqr } & \text { Adj Rsqr } & \text { Standard Error of Estimate } \\ 0.9995 & 0.9989 & 0.9987 & 3.1373\end{array}$

Coefficient Std. Error t $\quad$ P

$\begin{array}{lrrrr}\text { a } & 193.3807 & 2.2912 & 84.4018 & <0.0001 \\ \text { b } & -0.4918 & 0.0218 & -22.5888 & <0.0001 \\ \text { x0 } & 6.4266 & 0.0248 & 259.3023 & <0.0001 \\ \text { y0 } & 19.2942 & 1.6043 & 12.0265 & <0.0001\end{array}$

Analysis of Variance:

\begin{tabular}{lrrr} 
& \multicolumn{1}{c}{ DF } & \multicolumn{1}{c}{ SS } & \multicolumn{1}{c}{ MS } \\
Regression 4 & 353880.1428 & 88470.0357 \\
Residual & 12 & 118.1149 & 9.8429 \\
Total & 16 & 353998.2576 & 22124.8911
\end{tabular}

Corrected for the mean of the observations:

\begin{tabular}{lrrrrr} 
& \multicolumn{1}{c}{ DF } & SS & MS & F & P \\
Regression 3 & 111715.7644 & 37238.5881 & 3783.2927 & $<0.0001$ \\
Residual & 12 & 118.1149 & 9.8429 & & \\
Total & 15 & 111833.8792 & 7455.5919 & &
\end{tabular}

\section{Statistical Tests:}

Normality Test (Shapiro-Wilk) Passed $\quad(P=0.4057)$

W Statistic $=0.9443 \quad$ Significance Level $=0.0500$

Constant Variance Test $\quad$ Failed $(\mathrm{P}=<0.0001)$

Fit Equation Description:

[Variables]

$\mathrm{x}=\operatorname{col}(5)$

$\mathrm{y}=\operatorname{col}(6)$

reciprocal_y $=1 / \mathrm{abs}(\mathrm{y})$

reciprocal ysquare $=1 / \mathrm{y}^{\wedge} 2$

reciprocal_pred $=1 / \mathrm{abs}(\mathrm{f})$

reciprocal predsqr $=1 / \mathrm{f}^{\wedge} 2$

[Parameters]

$\mathrm{a}=\max (\mathrm{y})-\min (\mathrm{y}) "$ Auto $\{$ previous: 193.381\}\}

$\mathrm{b}=\mathrm{if}(\mathrm{xwtr}(\mathrm{x}, \mathrm{y}-\min (\mathrm{y}), .5) \diamond 0, \mathrm{xwtr}(\mathrm{x}, \mathrm{y}-\min (\mathrm{y}), .5) / 4,1)$ "Auto \{ \{previous: -0.491755$\}\}$

$\mathrm{x} 0=\mathrm{x} 50(\mathrm{x}, \mathrm{y}-\min (\mathrm{y}), .5)$ "Auto $\{$ previous: 6.42663$\}$ \}

y0 $=\min (\mathrm{y}) "$ Auto $\{\{$ previous: 19.2942$\}\}$

[Equation]

$\mathrm{f}=\mathrm{y} 0+\mathrm{a} /(1+\exp (-(\mathrm{x}-\mathrm{x} 0) / \mathrm{b}))$

fit $\mathrm{f}$ to $\mathrm{y}$ 


\section{4-POBA_Mannose_430nm_2}

\begin{tabular}{|c|c|c|c|c|c|c|}
\hline \multicolumn{7}{|c|}{ Nonlinear Regression } \\
\hline \multicolumn{7}{|c|}{$\begin{array}{l}\text { Data Source: Data } 1 \text { in All for POBA.JNB } \\
\text { Equation: Standard Curves, Four Parameter Logistic Curve } \\
\mathrm{f} 1=\min +(\max -\mathrm{min}) /\left(1+\mathrm{abs}(\mathrm{x} / \mathrm{EC} 50)^{\wedge} \text { Hillslope }\right) \\
\mathrm{f} 2=\min +(\max -\min )^{*}\left(\mathrm{abs}(\mathrm{x} / \mathrm{EC} 50)^{\wedge} \text { abs }(\text { Hillslope })\right) /\left(1+\left(\mathrm{abs}(\mathrm{x} / \mathrm{EC} 50)^{\wedge} \mathrm{abs}(\text { Hillslope })\right)\right) \\
\mathrm{f}=\mathrm{if}(\mathrm{x}<=0, \text { if }(\text { Hillslope }>0, \max , \mathrm{min}), \text { if }(\text { Hillslope }>0, \mathrm{f} 1, \mathrm{f} 2))\end{array}$} \\
\hline $\mathbf{R}$ & Rsqr & Adj Rs & St: & adard Erro & timate & \\
\hline \multirow[t]{2}{*}{0.9993} & 0.9987 & 0.9984 & 3.5 & & & \\
\hline & Co & efficient & Std. Error & $\mathbf{t}$ & $\mathbf{P}$ & VIF \\
\hline $\min$ & 16.34 & & 1.4381 & 11.3686 & $<0.0001$ & 1.5486 \\
\hline $\max$ & 211.58 & & 1.5130 & 139.8442 & $<0.0001$ & 1.4267 \\
\hline EC50 & 6.43 & & 0.0267 & 241.2231 & $<0.0001$ & 1.4760 \\
\hline Hillslope & 12.83 & & 0.5943 & 21.5934 & $<0.0001$ & 1.4604 \\
\hline
\end{tabular}

Analysis of Variance:

Uncorrected for the mean of the observations:

DF SS MS

Regression $4 \quad 354503.1801 \quad 88625.7950$

Residual $15 \quad 185.4685 \quad 12.3646$

$\begin{array}{llll}\text { Total } & 19 & 354688.6486 & 18667.8236\end{array}$

Corrected for the mean of the observations:

\begin{tabular}{lrrrrc} 
& DF & SS & MS & \multicolumn{1}{c}{ F } & P \\
Regression 3 & 141062.0020 & 47020.6673 & 3802.8557 & $<0.0001$ \\
Residual & 15 & 185.4685 & 12.3646 & & \\
Total & 18 & 141247.4705 & 7847.0817 & &
\end{tabular}

Statistical Tests:

PRESS $\quad 306.2209$

\begin{tabular}{|c|c|c|c|}
\hline \multicolumn{3}{|c|}{ Durbin-Watson Statistic } & 1.2026 Failed \\
\hline \multicolumn{3}{|c|}{ Normality Test } & $(\mathrm{P}=0.9899)$ \\
\hline \multicolumn{2}{|c|}{ K-S Statistic $=0.0980$} & \multicolumn{2}{|c|}{ Significance Level $=0.9899$} \\
\hline \multicolumn{2}{|c|}{ Constant Variance Test } & & $(\mathrm{P}=0.0077)$ \\
\hline \multicolumn{4}{|c|}{ Power of performed test with alpha $=0.0500: 1.0000$} \\
\hline \multicolumn{4}{|c|}{ Regression Diagnostics: } \\
\hline Row & Std. Res. & Stud. Res & Stud. Del. Res. \\
\hline 1 & 1.4634 & 1.6211 & 1.7245 \\
\hline 2 & 2.1066 & $2.3334<$ & $2.8245<$ \\
\hline 3 & -0.9170 & -1.0148 & -1.0159 \\
\hline 4 & -0.1987 & -0.2187 & -0.2116 \\
\hline 5 & -1.2372 & -1.3434 & -1.3837 \\
\hline
\end{tabular}


4-POBA_Sorbitol_430nm_1

Nonlinear Regression

Wednesday, September 21, 2011, 2:53:34 PM

Data Source: Data 1 in Titration Reports

Equation: Sigm oidal, Sigmoid, 4 Parameter

$\mathrm{f}=\mathrm{y} 0+\mathrm{a} /(1+\exp (-(\mathrm{x}-\mathrm{x} 0) / \mathrm{b}))$

\section{R Rsqr Adj Rsqr Standard Error of Estimate \\ $\begin{array}{llll}0.9997 & 0.9995 & 0.9994 & 2.7690\end{array}$}

\section{Coefficient Std. Error t $\quad$ P}

$\begin{array}{lrrrr}\text { a } & 242.5169 & 1.9306 & 125.6183 & <0.0001 \\ \text { b } & -0.4695 & 0.0150 & -31.3599 & <0.0001 \\ \text { x0 } & 6.5998 & 0.0172 & 384.5378 & <0.0001 \\ \text { y0 } & 8.6625 & 1.3844 & 6.2572 & <0.0001\end{array}$

Analysis of Variance:

\begin{tabular}{lrrr} 
& \multicolumn{1}{c}{ DF } & \multicolumn{1}{c}{ SS } & \multicolumn{1}{c}{ MS } \\
Regression 4 & 498423.6230 & 124605.9057 \\
Residual & 12 & 92.0076 & 7.6673 \\
Total & 16 & 498515.6306 & 31157.2269
\end{tabular}

Corrected for the mean of the observations:

\begin{tabular}{lrrrrr} 
& DF & SS & MS & \multicolumn{1}{c}{ F } & P \\
Regression 3 & 181431.8211 & 60477.2737 & 7887.6865 & $<0.0001$ \\
Residual & 12 & 92.0076 & 7.6673 & & \\
Total & 15 & 181523.8287 & 12101.5886 & &
\end{tabular}

Statistical Tests:

Normality Test (Shapiro-Wilk) Passed $\quad(P=0.2562)$

W Statistic $=0.9314 \quad$ Significance Level $=0.0500$

Constant Variance Test Failed $(\mathrm{P}=0.0213)$

Fit Equation Description:

[Variables]

$\mathrm{x}=\operatorname{col}(7)$

$\mathrm{y}=\operatorname{col}(8)$

reciprocal_y $=1 / \mathrm{abs}(\mathrm{y})$

reciprocal ysquare $=1 / \mathrm{y}^{\wedge} 2$

reciprocal_pred $=1 / \mathrm{abs}(\mathrm{f})$

reciprocal_predsqr $=1 / \mathrm{f}^{\wedge} 2$

[Parameters]

$\mathrm{a}=\max (\mathrm{y})-\min (\mathrm{y}) "$ Auto $\{\{$ previous: 242.517$\}$ \}

$\mathrm{b}=\mathrm{if}(\mathrm{xwtr}(\mathrm{x}, \mathrm{y}-\min (\mathrm{y}), .5) \diamond 0, \operatorname{xwtr}(\mathrm{x}, \mathrm{y}-\min (\mathrm{y}), .5) / 4,1)$ "Auto $\{\{$ previous: -0.469452$\}\}$

$\mathrm{x} 0=\mathrm{x} 50(\mathrm{x}, \mathrm{y}-\mathrm{min}(\mathrm{y}), .5)$ "Auto \{\{previous: 6.59985$\}\}$

y0 $=\min (y) "$ Auto $\{\{$ previous: 8.66252$\}\}$

[Equation]

$\mathrm{f}=\mathrm{y} 0+\mathrm{a} /(1+\exp (-(\mathrm{x}-\mathrm{x} 0) / \mathrm{b}))$

fit $\mathrm{f}$ to $\mathrm{y}$ 
4-POBA_Sorbitol_430nm_2

\section{Nonlinear Regression}

Data Source: Data 1 in All for POBA.JNB

Equation: Standard Curves, Four Parameter Logistic Curve

$\mathrm{fl}=\min +(\max -\min ) /\left(1+\operatorname{abs}(\mathrm{x} / \mathrm{EC} 50)^{\wedge}\right.$ Hillslope $)$

$\mathrm{f} 2=\min +(\max -\min )^{*}\left(\operatorname{abs}(\mathrm{x} / \mathrm{EC} 50)^{\wedge} \mathrm{abs}(\right.$ Hillslope $\left.)\right) /\left(1+\left(\operatorname{abs}(\mathrm{x} / \mathrm{EC} 50)^{\wedge} \mathrm{abs}(\right.\right.$ Hillslope $\left.\left.)\right)\right)$

$\mathrm{f}=$ if $(\mathrm{x}<=0$, if(Hillslope $>0$, max, min), if(Hillslope $>0, \mathrm{f} 1, \mathrm{f} 2)$ )

\section{R Rsqr Adj Rsqr Standard Error of Estimate}

$\begin{array}{llll}0.9994 & 0.9988 & 0.9986 & 4.2373\end{array}$

\begin{tabular}{|c|c|c|c|c|c|}
\hline & Coefficient & Std. Error & $\mathbf{t}$ & $\mathbf{P}$ & VIF \\
\hline $\min$ & 9.3925 & 1.6585 & 5.6632 & $<0.0001$ & 1.4330 \\
\hline $\max$ & 249.7408 & 1.7338 & 144.0402 & $<0.0001$ & 1.3259 \\
\hline EC50 & 6.5847 & 0.0251 & 262.4509 & $<0.0001$ & 1.3919 \\
\hline Hillslope & 14.4402 & 0.6898 & 20.9351 & $<0.0001$ & 1.3789 \\
\hline
\end{tabular}

Analysis of Variance:

Uncorrected for the mean of the observations:

DF SS MS

Regression $4 \quad 498788.4676 \quad 124697.1169$

$\begin{array}{llrr}\text { Residual } 15 & 269.3226 & 17.9548\end{array}$

$\begin{array}{llll}\text { Total } & 19 & 499057.7902 & 26266.1995\end{array}$

Corrected for the mean of the observations:

$\begin{array}{lrrrrr} & \text { DF } & \text { SS } & \text { MS } & \text { F } & \text { P } \\ \text { Regression } 3 & 222945.1680 & 74315.0560 & 4138.9981 & <0.0001 \\ \text { Residual } & 15 & 269.3226 & 17.9548 & & \\ \text { Total } & 18 & 223214.4906 & 12400.8050 & & \end{array}$

Statistical Tests:

PRESS $\quad 393.0862$

Durbin-Watson Statistic $\quad 1.0801$ Failed

Normality Test $\quad$ Passed $(P=0.7701)$

K-S Statistic $=0.1474 \quad$ Significance Level $=0.7701$

Constant Variance Test $\quad$ Passed $(P=0.1712)$

Power of performed test with alpha $=0.0500: 1.0000$

Regression Diagnostics:

$\begin{array}{lccc}\text { Row } & \text { Std. Res. } & \text { Stud. Res. } & \text { Stud. Del. Res. } \\ 1 & 1.0469 & 1.1474 & 1.1606 \\ 2 & 0.4648 & 0.5094 & 0.4965 \\ 3 & 0.2514 & 0.2754 & 0.2668 \\ 4 & 1.2602 & 1.3792 & 1.4259 \\ 5 & -0.6653 & -0.7241 & -0.7121\end{array}$


4-POBA_Tagatose_430nm_1

Nonlinear Regression

Wednesday, September 21, 2011, 2:55:12 PM

Data Source: Data 1 in Titration Reports

Equation: Sigm oidal, Sigmoid, 4 Parameter

$\mathrm{f}=\mathrm{y} 0+\mathrm{a} /(1+\exp (-(\mathrm{x}-\mathrm{x} 0) / \mathrm{b}))$

$\begin{array}{llll}\text { R } & \text { Rsqr } & \text { Adj Rsqr } & \text { Standard Error of Estimate } \\ 0.9998 & 0.9996 & 0.9994 & 2.5450\end{array}$

Coefficient Std. Error t P

$\begin{array}{lrrrr}\text { a } & 237.8174 & 1.7744 & 134.0243 & <0.0001 \\ \text { b } & -0.4706 & 0.0141 & -33.4481 & <0.0001 \\ \text { x0 } & 6.5720 & 0.0158 & 415.6613 & <0.0001 \\ \text { y0 } & 8.8595 & 1.2716 & 6.9672 & <0.0001\end{array}$

Analysis of Variance:

\begin{tabular}{lrrr} 
& \multicolumn{1}{c}{ DF } & \multicolumn{1}{c}{ SS } & \multicolumn{1}{c}{ MS } \\
Regression 4 & 482109.9243 & 120527.4811 \\
Residual & 12 & 77.7221 & 6.4768 \\
Total & 16 & 482187.6463 & 30136.7279
\end{tabular}

Corrected for the mean of the observations:

$\begin{array}{lrrrrr} & \text { DF } & \text { SS } & \text { MS } & \text { F } & \text { P } \\ \text { Regression } 3 & 172906.9226 & 57635.6409 & 8898.7299 & <0.0001 \\ \text { Residual } 12 & 77.7221 & 6.4768 & & \\ \text { Total } & 15 & 172984.6447 & 11532.3096 & & \end{array}$

\section{Statistical Tests:}

Normality Test (Shapiro-Wilk) Passed $(\mathrm{P}=0.2912)$

W Statistic $=0.9349 \quad$ Significance Level $=0.0500$

Constant Variance Test Failed $(\mathrm{P}=0.0238)$

Fit Equation Description:

[Variables]

$\mathrm{x}=\operatorname{col}(9)$

$\mathrm{y}=\operatorname{col}(10)$

reciprocal $\mathrm{y}=1 / \mathrm{abs}(\mathrm{y})$

reciprocal_ysquare $=1 / \mathrm{y}^{\wedge} 2$

reciprocal pred $=1 / \mathrm{abs}(\mathrm{f})$

reciprocal_predsqr $=1 / \mathrm{f}^{\wedge} 2$

[Parameters]

$\mathrm{a}=\max (\mathrm{y})-\min (\mathrm{y}) "$ Auto $\{$ previous: 237.817$\}\}$

$\mathrm{b}=\mathrm{if}(\mathrm{xwtr}(\mathrm{x}, \mathrm{y}-\min (\mathrm{y}), .5) \circlearrowright 0, \mathrm{xwtr}(\mathrm{x}, \mathrm{y}-\min (\mathrm{y}), .5) / 4,1)$ "Auto \{\{previous: -0.470598$\}\}$

$\mathrm{x} 0=\mathrm{x} 50(\mathrm{x}, \mathrm{y}-\mathrm{min}(\mathrm{y}), 5) "$ Auto $\{$ previous: 6.57201$\}\}$

y0 $=\min (\mathrm{y}) "$ Auto $\{\{$ previous: 8.85953$\}\}$

[Equation]

$\mathrm{f}=\mathrm{y} 0+\mathrm{a} /(1+\exp (-(\mathrm{x}-\mathrm{x} 0) / \mathrm{b}))$

fit $\mathrm{f}$ to $\mathrm{y}$ 


\section{4-POBA_Tagatose_430nm_2}

\section{Nonlinear Regression}

Data Source: Data 1 in All for POBA.JNB Equation: Standard Curves, Four Parameter Logistic Curve $\mathrm{fl}=\min +(\max -\min ) /\left(1+\operatorname{abs}(\mathrm{x} / \mathrm{EC} 50)^{\wedge}\right.$ Hillslope $)$

$\mathrm{f} 2=\min +(\max -\mathrm{min})^{*}\left(\operatorname{abs}(\mathrm{x} / \mathrm{EC} 50)^{\wedge} \mathrm{abs}(\right.$ Hillslope $\left.)\right) /\left(1+\left(\operatorname{abs}(\mathrm{x} / \mathrm{EC} 50)^{\wedge} \mathrm{abs}(\right.\right.$ Hillslope $\left.\left.)\right)\right)$ $\mathrm{f}=\mathrm{if}(\mathrm{x}<=0$, if(Hillslope $>0, \max , \mathrm{min})$, if(Hillslope $>0, \mathrm{f} 1, \mathrm{f} 2)$ )

\section{R Rsqr Adj Rsqr $\quad$ Standard Error of Estimate}

$\begin{array}{llll}0.9997 & 0.9994 & 0.9993 & 2.9637\end{array}$

\begin{tabular}{|c|c|c|c|c|c|}
\hline & Coefficient & Std. Error & $\mathbf{t}$ & $\mathbf{P}$ & VIF \\
\hline $\min$ & 7.5522 & 1.1740 & 6.4328 & $<0.0001$ & 1.4501 \\
\hline $\max$ & 245.5562 & 1.2201 & 201.2518 & $<0.0001$ & 1.3446 \\
\hline EC50 & 6.5654 & 0.0176 & 371.9841 & $<0.0001$ & 1.3989 \\
\hline Hillslope & 13.9610 & 0.4634 & 30.1293 & $<0.0001$ & 1.3682 \\
\hline
\end{tabular}

Analysis of Variance:

Uncorrected for the mean of the observations:

\begin{tabular}{lcrr} 
& \multicolumn{1}{c}{ DF } & \multicolumn{1}{c}{ SS } & \multicolumn{1}{c}{ MS } \\
Regression 4 & 482259.5182 & 120564.8796 \\
Residual & 15 & 131.7504 & 8.7834 \\
Total & 19 & 482391.2687 & 25389.0141
\end{tabular}

Corrected for the mean of the observations:

\begin{tabular}{lrrrrr} 
& \multicolumn{1}{c}{ DF } & SS & MS & \multicolumn{1}{c}{ F } & P \\
Regression 3 & 216070.7186 & 72023.5729 & 8199.9995 & $<0.0001$ \\
Residual 15 & 131.7504 & 8.7834 & & \\
Total & 18 & 216202.4691 & 12011.2483 & &
\end{tabular}

Statistical Tests:

PRESS $\quad 251.6510$

Durbin-Watson Statistic $\quad 2.2183$ Passed

Normality Test $\quad$ Passed $(\mathrm{P}=0.9933)$

K-S Statistic $=0.0948 \quad$ Significance Level $=0.9933$

Constant Variance Test $\quad$ Failed $\quad(\mathrm{P}=<0.0001)$

Power of performed test with alpha $=0.0500: 1.0000$

Regression Diagnostics:

$\begin{array}{lccc}\text { Row } & \text { Std. Res. } & \text { Stud. Res. } & \text { Stud. Del. Res. } \\ 1 & 1.0790 & 1.1840 & 1.2014 \\ 2 & 0.9096 & 0.9981 & 0.9979 \\ 3 & -0.7531 & -0.8262 & -0.8170 \\ 4 & 1.6475 & 1.8040 & 1.9696 \\ 5 & -1.6783 & -1.8272 & -2.0021<\end{array}$

